\title{
Die Reproduktion sozialer Ungleichheiten in der Freiwilligenarbeit
}

Theoretische Perspektiven und empirische Analysen zur sozialen Schließung und Hierarchisierung in der Freiwilligenarbeit

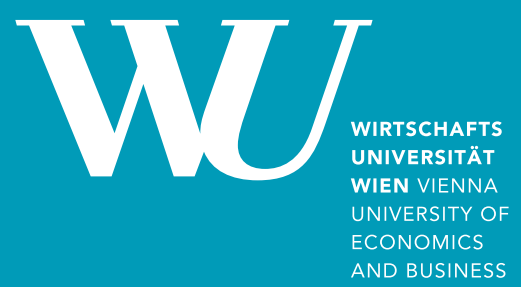


Paul Rameder widmet sich der Frage, in welcher Form und in welchen Bereichen die Freiwilligenarbeit einen Beitrag zur Genese und Reproduktion sozialer Ungleichheiten leistet. Durch die Aura der Freiwilligkeit und Uneigennützigkeit entziehen sich die sozial nachteiligen Effekte der Freiwilligenarbeit der öffentlichen Wahrnehmung und expliziten Kritik. Die multivariaten Analysen von Mikrozensusdaten aus Österreich zeigen, dass der Zugang zur Freiwilligenarbeit in hohem Maße durch die Ressourcenausstattung der Individuen geprägt ist. Auch die Funktionsverteilung innerhalb der Freiwilligenarbeit reproduziert die ungleichen sozialen Machtverhältnisse. So tragen die Mechanismen der sozialen Schließung und Hierarchisierung auch in den Feldern der Freiwilligenarbeit zu einer Verfestigung gesellschaftlicher Ungleichheiten bei.

Paul Rameder ist wissenschaftlicher Mitarbeiter am Institut für Nonprofit Management der Wirtschaftsuniversität Wien. Er lehrt und forscht zu den Themengebieten Freiwilligenarbeit, Freiwilligenmanagement, Service Learning sowie soziale Ungleichheit. 
Die Reproduktion sozialer Ungleichheiten in der Freiwilligenarbeit 


\section{Forschungsergebnisse der WU Wirtschaftsuniversität Wien}

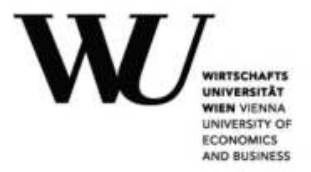

Band 67 


\section{Die Reproduktion sozialer Ungleichheiten in der Freiwilligenarbeit}

Theoretische Perspektiven und empirische Analysen zur sozialen Schließung und Hierarchisierung in der Freiwilligenarbeit 


\title{
Bibliografische Information der Deutschen Nationalbibliothek
}

Die Deutsche Nationalbibliothek verzeichnet diese Publikation in der Deutschen Nationalbibliografie; detaillierte bibliografische Daten sind im Internet über http://dnb.d-nb.de abrufbar.

Gefördert durch die WU Wirtschaftsuniversität Wien.

\author{
Umschlaggestaltung: \\ Atelier Platen, nach einem Entwurf \\ von Werner Weißhappl. \\ Universitätslogo der WU Wirtschaftsuniversität Wien: \\ Abdruck mit freundlicher Genehmigung \\ der WU Wirtschaftsuniversität Wien.
}

ISSN 1613-3056

ISBN 978-3-631-66434-6 (Print)

E-ISBN 978-3-653-05595-5 (E-Book)

DOI 10.3726/978-3-653-05595-5

(c) Peter Lang GmbH

Internationaler Verlag der Wissenschaften

Frankfurt am Main 2015

Alle Rechte vorbehalten.

PL Academic Research ist ein Imprint der Peter Lang $\mathrm{GmbH}$.

Peter Lang - Frankfurt am Main · Bern · Bruxelles · New York ·

Oxford $\cdot$ Warszawa $\cdot$ Wien

Das Werk einschließlich aller seiner Teile ist urheberrechtlich geschützt. Jede Verwertung außerhalb der engen Grenzen des

Urheberrechtsgesetzes ist ohne Zustimmung des Verlages unzulässig und strafbar. Das gilt insbesondere für Vervielfältigungen, Übersetzungen, Mikroverfilmungen und die Einspeicherung und Verarbeitung in elektronischen Systemen.

Diese Publikation wurde begutachtet. www.peterlang.com 
für Birgit und Alma 



\title{
Inhaltsverzeichnis
}

\author{
Abbildungsverzeichnis
}

Tabellenverzeichnis

Einleitung 19

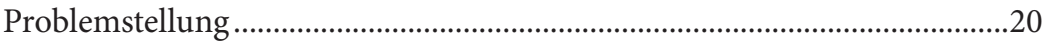

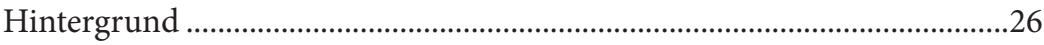

Aufbau der Arbeit............................................................................................

(I) Theoretische Perspektiven auf soziale Ungleichheit und Freiwilligenarbeit.

(I) 1. Soziale Ungleichheit - Definitionen, Formen

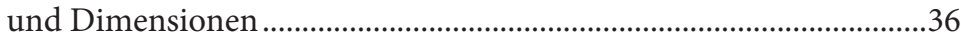

(I) 1.1. Definitionen sozialer Ungleichheit ........................................... 36

(I) 1.2. Formen sozialer Ungleichheit.................................................... 38

(I) 1.3. Dimensionen sozialer Ungleichheit........................................... 39

(I) 2. Theoretische Perspektiven zu den Struktur- und Kontextebenen sozialer Ungleich heit ....................................................42

(I) 2.1 Strukturebenen sozialer Ungleichheit ..................................... 42

(I) 2.2. Kontextebenen sozialer Ungleichheit ........................................ 45

(I) 3. Freiwilligenarbeit und soziale Ungleichheit..........................................51

(I) 3.1. Freiwilligenarbeit als Determinante, Dimension und Auswirkung sozialer Ungleichheit ...................................... 51

(I) 3.1.1. Freiwilligenarbeit als Determinante sozialer Ungleichheit ................................................................ 52

(I) 3.1.2. Freiwilligenarbeit als Dimension sozialer Ungleich heit ................................................................ 53

(I) 3.1.3. Freiwilligenarbeit als Auswirkung sozialer Ungleichheit 56 
(I) 3.2. Freiwilligenarbeit als Kontextebene der Genese und Reproduktion sozialer Ungleichheit

(I) 4. Theoretische Perspektiven zur sozialen Schließung und Hierarisierung in der Freiwilligenarbeit. 59

(I) 4.1. Grundlagen der Sozialtheorie Pierre Bourdieus ....................... 61

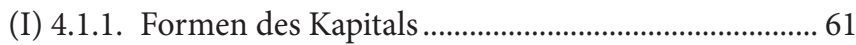

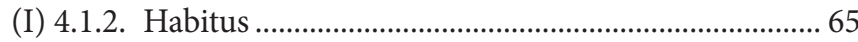

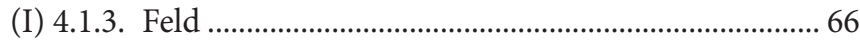

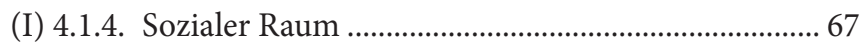

(I) 4.2. Freiwilligenarbeit und Ehrenamt in der Theorie Pierre Bourdieus 68

(I) 4.2.1. Soziale Schließung: Ökonomisches Kapital als Voraussetzung für Freiwilliges Engagement...... 70

(I) 4.2.2. Hierarchisierung: Interne Arbeitsteilung und die Verschleierung von Ausbeutungsverhältnissen. 72

(I) 4.2.3. Altruismus und Unbezahlbarkeit als Illusio im Feld der Freiwilligenarbeit 74

(I) 4.2.4. Der Lohn der Freiwilligenarbeit: Die ungleiche Verteilung von Anerkennung und Ehre.

(I) 5. Fazit: (Re)-Produktion sozialer Ungleichheiten in der Freiwilligenarbeit durch Soziale Schließung und Hierarchisierung .80

\section{(II) Stand der Forschung zu den Determinanten} und Auswirkungen der Freiwilligenarbeit .85

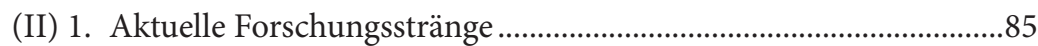

(II) 2. Einflussfaktoren auf Freiwilligenarbeit................................................8

(II) 3. Soziale Merkmale als Determinanten des Zugangs zur Freiwilligenarbeit

(II) 3.1. Zugeschriebene Merkmale als Determinanten des Zugangs zur Freiwilligenarbeit 94

(II) 3.1.1. Geschlecht .......................................................... 94

(II) 3.1.2. Alter 
(II) 3.1.3. Ethnische und geographische Herkunft.......... 100

(II) 3.1.4. Soziale Herkunft ................................................... 100

(II) 3.2. Erworbene Merkmale als Determinanten des Zugangs zur Freiwilligenarbeit..................................... 102

(II) 3.2.1. Formaler Bildungsgrad........................................ 102

(II) 3.2.2. Erwerbstätigkeit und berufliche Stellung........ 105

(II) 3.2.3. Prestige und Status ................................................. 107

(II) 3.2.4. Einkommen und Vermögen ............................... 111

(II) 3.2.5. Soziales Kapital ....................................................... 113

(II) 4. Stand der Forschung zu den Auswirkungen der Freiwilligenarbeit.

(II) 4.1.1. Effekte auf Erwerbstätigkeit, Beruf und Einkommen........................................ 115

(II) 4.1.2. Effekte auf Gesundheit und Lebenszufriedenheit.

\section{(III) Empirische Analysen zur sozialen Schließung} und Hierarchisierung in der Freiwilligenarbeit in Österreich

(III) 1. Fragestellungen für die empirischen Analysen 121

(III) 2. Methodik

(III) 2.1. Quantitative empirische Freiwilligenforschung........... 122

(III) 2.2. Beschreibung des Datensatzes........................................ 128

(III) 2.3. Variablenbeschreibung.................................................. 130

(III) 2.3.1. Abhängige Variablen ..................................... 130

(III) 2.3.2. Erklärende Variablen..................................... 132

(III) 2.5. Deskriptive Statistik....................................................... 133

(III) 2.6. Statistische Analyseverfahren.......................................... 134

(III) 2.6.1. Deskriptive und bivariate Analyseverfahren .......................................... 135

(III) 2.6.2. Multivariate Analyseverfahren.................... 135

(III) 3. Analysestrategie …....................................................................... 136 
(III) 4.1. Bivariate Analysen ................................................................ 138

(III) 4.1.1. Geschlecht .............................................................. 138

(III) 4.1.2. Alter........................................................................... 139

(III) 4.1.3. Ethnische Herkunft ............................................... 140

(III) 4.1.4. Staatsbürgerschaft................................................... 140

(III) 4.1.5. Bildungsgrad .......................................................... 141

(III) 4.1.6. Erwerbstätigkeit..................................................... 142

(III) 4.1.7. Wohnrechtsverhältnis ............................................. 142

(III) 4.1.8. Familienstand........................................................... 143

(III) 4.1.9. Zusammenfassung der Ergebnisse ...................... 143

(III) 4.2. Multivariate Analysen zur sozialen Schließung in der Freiwilligenarbeit .................................................... 145

(III) 4.2.1. Determinanten des Zugangs zur Freiwilligenarbeit.................................................... 145

(III) 4.2.2. Determinanten des Zugangs zur Freiwilligenarbeit und Erwerbsarbeit im Vergleich ............................................................. 148

(III) 4.2.3. Determinanten des Zugangs von Frauen und Männern im Vergleich.................................... 150

(III) 4.2.4. Determinanten von Mehrfachengagement....... 154

(III) 4.2.5. Determinanten des Zugangs der bildungsfernsten Schicht 156

(III) 4.2.6. Subjektive Einschätzung der Barrieren der Freiwilligenarbeit ............................................ 158

(III) 4.2.7. Zusammenfassung der Ergebnisse zur sozialen Schließung.....

(III) 4.3. Multivariate Analysen zur internen Hierarchisierung in der Freiwilligenarbeit

(III) 4.3.1. Determinanten des Zugangs zu Positionen mit Leitungsfunktion

(III) 4.3.2. Determinanten des Zugangs zu

Positionen mit Leitungsfunktion in der

Freiwilligenarbeit und Erwerbsarbeit im

Vergleich 
(III) 4.3.3. Mehrfachengagement und der Zugang zu Positionen mit Leitungsfunktion 168

(III) 4.3.4. Determinanten des Zugangs von Frauen und Männer zu Positionen mit Leitungsfunktion im Vergleich

(III) 4.3.5. Beruflicher Status (ISEI) als Determinante bei Männern und Frauen

(III) 4.3.6. „Paradox der Gestreßtheit“: Zum Zusammenhang von Arbeitszeit und Freiwilligenarbeit.

(III) 4.3.7. Zusammenfassung der Ergebnisse zur Hierarchisierung 176

(III) 4.4 Multivariate Analysen zu ausgewählten Feldern der Freiwilligenarbeit

(III) 4.4.1. Ausgewählte Bereiche für die feldspezifischen Analysen 178

(III) 4.4.2. Bivariate Analysen zur Über- und Unterrepräsentanz der sozialen Merkmalsgruppen in den Feldern der Freiwilligenarbeit.

(III) 4.4.3. Multivariate Regressionsanalysen zur sozialen Schließung in den Feldern

(III) 4.4.4. Multivariate Korrespondenzanalysen zur Hierarchisierung in den Feldern

(III) 4.4.5. Positionen im Feld des Sozialen und der Gesundheit

(III) 4.4.6. Positionen im Feld der Kirche und Religion ..... 196

(III) 4.4.7. Positionen im Feld des Sports und der Bewegung.

(III) 4.4.8. Positionen im Feld der Katastrophenhilfsund Rettungsdienste

(III) 4.4.9. Zusammenfassung der Ergebnisse zu den ausgewählten Feldern

(III) 5. Zusammenfassung der zentralen

empirischen Ergebnisse 204

(III) 5.1. Ergebnisse zur sozialen Schließung 204 
(III) 5.2. Ergebnisse zur internen Hierarchisierung ........................ 205

(III) 5.3. Ergebnisse zur sozialen Schließung und

Hierarchisierung in den Feldern ......................................... 206

(III) 6. Limitationen ......................................................................................... 207

(IV) Schlussfolgerungen .............................................................................. 209

(IV) 1. Relevanz der Ergebnisse für die weitere

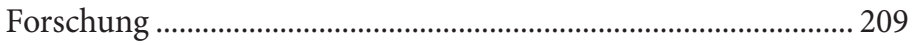

(IV) 2. Relevanz der Ergebnisse auf individueller, organisationaler, politischer und gesellschaftlicher Ebene.......................................... 211

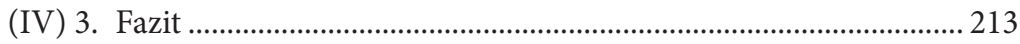

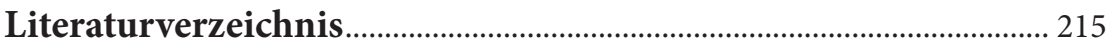

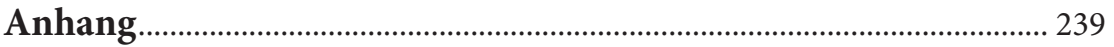

Anhang 1: Theoretische Perspektiven................................................... 239

Anhang 2: Empirische Analysen ............................................................. 240

Anhang 3: Fragebogen der empirischen Erhebung .............................. 256 


\section{Abbildungsverzeichnis}

Abbildung 1: Schematische Darstellung des Volunteer Process Model ...............30

Abbildung 2: Dimensionen strukturierter sozialer Ungleichheit .......................39

Abbildung 3: Strukturebenen sozialer Ungleichheit ..............................................43

Abbildung 4: Von Heterogenitäten zu Ungleichheiten ........................................47

Abbildung 5: Formen kumulativer Vor- und Nachteile ..........................................50

Abbildung 6: Theoretische Verortung von Freiwilligenarbeit im Kontext sozialer Ungleichheit .....................................................52

Abbildung 7: Freiwilligenarbeit als Determinante sozialer Ungleichheit..........53

Abbildung 8: Freiwilligenarbeit als Dimension sozialer Ungleichheit...............54

Abbildung 9: Vereinfachte Darstellung des sozialen Raums bei Bourdieu ......68

Abbildung 10: Modell der Reproduktion sozialer Ungleichheit in der Freiwilligenarbeit.......................................................................81

Abbildung 11: A hybrid conceptual framework of volunteering ...........................86

Abbildung 12: Einflussfaktoren auf Freiwilliges Engagement aus individueller Perspektive.............................................................89

Abbildung 13: Moderator- und Mediatorvariablen des Zusammenhangs von Bildungsgrad und Freiwilligenarbeit...................................... 104

Abbildung 14: Geschlecht und Alter.................................................................... 139

Abbildung 15: Geburtsland und Staatsbürgerschaft ............................................ 140

Abbildung 16: Bildungsgrad und Erwerbstätigkeit............................................... 141

Abbildung 17: Wohnrechtsverhältnis und Familienstand .................................. 143

Abbildung 18: Zusammenhang der Wochenarbeitszeit (Std.) in der Erwerbsarbeit (Referenzwoche) mit der Leitungsfunktion in der Freiwilligenarbeit............................................................... 175

Abbildung 19: Über- und Unterrepräsentation nach Geschlechtern in den Feldern 
Abbildung 20: Über- und Unterrepräsentation nach Altersgruppen in den Feldern

Abbildung 21: Über- und Unterrepräsentation nach Geburtsland in den Feldern.

Abbildung 22: Über- und Unterrepräsentation nach Staatsbürgerschaft in den Feldern

Abbildung 23: Über- und Unterrepräsentation nach Bildungsschicht in den Feldern

Abbildung 24: Über- und Unterrepräsentation nach Erwerbstätigkeit in den Feldern

Abbildung 25: Über- und Unterrepräsentation nach Funktion im Beruf in den Feldern

Abbildung 26: Über- und Unterrepräsentation nach

Wohnrechtsverhältnis in den Feldern.

Abbildung 27: Multiple Korrespondenzanalyse - Positionen in der Freiwilligenarbeit im Feld des Sozialen und der Gesundheit.

Abbildung 28: Multiple Korrespondenzanalyse - Positionen in der Freiwilligenarbeit im Feld der Kirche und Religion 195

Abbildung 29: Multiple Korrespondenzanalyse: Positionen in der Freiwilligenarbeit im Feld des Sports und der Bewegung........ 200

Abbildung 30: Multiple Korrespondenzanalyse: Positionen in der Freiwilligenarbeit im Feld der Katastrophenhilfsund Rettungsdienste. 


\section{Tabellenverzeichnis}

Tabelle 1: Beteiligungsquoten von Frauen und Männern ohne bzw. mit

Kindern im Haushalt

Tabelle 2: Beteiligungsquoten von Frauen und Männern nach

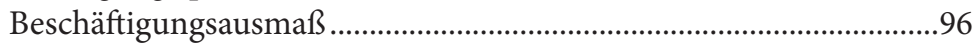

Tabelle 3: Beteiligungsstruktur nach Geschlecht in den Bereichen der formellen Freiwilligenarbeit .........................................................98

Tabelle 4: Formelle Freiwilligenarbeit - Ausgeübte Funktion

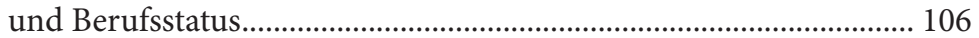

Tabelle 5: Formelle Freiwilligenarbeit - Vergleich des sozioökonomischen Status (ISEI) erwerbstätiger Personen........................ 110

Tabelle 6: Beteiligungsquote nach Haushaltseinkommen..................................... 112

Tabelle 7: Beteiligungsquoten formeller Freiwilligenarbeit nach

Bereichen in Österreich, Deutschland und der Schweiz ................... 126

Tabelle 8: Tätigkeitsbereiche der Freiwilligenarbeit ......................................... 128

Tabelle 9: Erhebungsdesign.................................................................................. 129

Tabelle 10: Erklärende Variablen - Merkmale und Variablenausprägung ...... 132

Tabelle 11: Deskriptive Statistik zu den abhängigen und erklärenden Variablen

Tabelle 12: Übersicht zu den bivariaten Ergebnissen zur sozialen Schließung

Tabelle 13: Logistische Regression - Einflussfaktoren auf den Zugang zur formellen Freiwilligenarbeit

Tabelle 14: Logistische Regression - Determinanten des Zugangs zur Erwerbsarbeit und der Freiwilligenarbeit.

Tabelle 15: Logistische Regression - Formelle Freiwilligenarbeit und der Einfluss der Leitungsfunktion im Beruf getrennt nach Frauen und Männer.

Tabelle 16: Logistische Regression - Formelle Freiwilligenarbeit: Frauen und Männer (inkl. Haushaltsdaten) 
Tabelle 17: Häufigkeiten von Mehrfachengagement - Anzahl der Organisationen

Tabelle 18: Lineare Regression - Mehrfachengagement in der Freiwilligenarbeit.

Tabelle 19: Logistische Regression - Formelle Freiwilligenarbeit von

Personen mit höchstens Pflichtschulabschluss getrennt nach Frauen und Männer.

Tabelle 20: Gründe sich nicht ehrenamtlich bzw. freiwillig zu engagieren.

Tabelle 21: Logistische Regression - Gründe für kein freiwilliges Engagement. 160

Tabelle 22: Logistische Regression - Leitende Funktion in der Freiwilligenarbeit

Tabelle 23: Logistische Regression - Determinanten des Zugangs zu Positionen mit Leitungsfunktion in der Erwerbsarbeit und der Freiwilligenarbeit

Tabelle 24: Logistische Regression - Leitenden Funktion in der Freiwilligenarbeit (inkl. Mehrfachengagement).

Tabelle 25: Logistische Regression - Leitende Funktion in der Freiwilligenarbeit getrennt nach Frauen und Männer. 170

Tabelle 26: Logistische Regression - Leitende Funktion in der Freiwilligenarbeit der Erwerbstätigen getrennt nach Frauen und Männer.

Tabelle 27: Beteiligungsquoten - Formelle Freiwilligenarbeit und Leitungsfunktion in der Freiwilligenarbeit nach Teilzeit- oder Vollzeitbeschäftigung

Tabelle 28: Logistische Regression - Einfluss von Beschäftigungsausmaß und Geschlecht beim Zugang zu leitenden Positionen in der Freiwilligenarbeit

Tabelle 29: Normale Wochenarbeitsstunden in der Erwerbsarbeit von Freiwilligen mit und ohne Leitungsfunktion im Vergleich. 175

Tabelle 30: Tätigkeitsbereiche der Freiwilligenarbeit 178

Tabelle 31: Logistische Regression - Determinanten der formelle Freiwilligenarbeit in den vier ausgewählten Feldern 
Tabelle 32: Variablen, Kategorien und deren Ausprägung (absolute und relative Häufigkeiten) in den Feldern.

Tabelle 33: Geschlechterverteilung in den Leitungsgremien der österreichischen Sportdachverbände

Tabelle 34: Inequality Mechanism and their Interactive Dynamics 239

Tabelle 35: Equality Mechanisms 239

Tabelle 36: Beteiligungsquoten und Beteiligungsstruktur..... 240

Tabelle 37: Beteiligungsquoten - Formelle Freiwilligenarbeit und Leitungsfunktion in der Freiwilligenarbeit nach dem Lebensunterhalt

Tabelle 38: Übersichtstabelle zum Vergleich der Beteiligungsstruktur der ausgewählten Felder.

Tabelle 39: Beteiligungsstruktur in der formellen Freiwilligenarbeit 243

Tabelle 40: Beteiligungsstruktur im Feld des Sozialen und der Gesundheit.

Tabelle 41: Beteiligungsstruktur im Feld der Kirche und Religion. 245

Tabelle 42: Beteiligungsstruktur im Feld des Sports und der Bewegung......... 246

Tabelle 43: Beteiligungsstruktur im Feld der Katastrophenhilfs- und Rettungsdienste.

Tabelle 44: Bedeutung der Kapitalien als Barriere für den Zugang zur Freiwilligenarbeit nach sozialen Merkmalsgruppen

Tabelle 45: Logistische Regression - Determinanten des Zugangs zur Freiwilligenarbeit (inkl. Erwerbsarbeit und Leitungsfunktion) .... 251

Tabelle 46: Logistische Regression - Determinanten des Zugangs zu leitenden Positionen in der Freiwilligenarbeit.

Tabelle 47: Logistische Regression - Determinanten des Zugangs zur Erwerbsarbeit (inkl. Freiwilligenarbeit) 253

Tabelle 48: Logistische Regression - Determinanten des Zugangs zu leitenden Positionen in der Erwerbsarbeit. 254

Tabelle 49: Logistische Regression - Einfluss der Freiwilligenarbeit in den verschiedenen Bereichen auf die berufliche Position. 



\title{
Einleitung
}

\author{
„Ein unerkanntes Gesetz ist \\ wie Natur, ist Schicksal [...]; \\ ein erkanntes Gesetz erscheint \\ als Möglichkeit von Freiheit" \\ (Bourdieu 2004: 44).
}

Die vorliegende Arbeit hat sich zum Ziel gesetzt, der Frage nach den Beiträgen der Freiwilligenarbeit bei der Entstehung und der Reproduktion sozialer Ungleichheit nachzugehen. Diese kritische Perspektive will begründet werden. Die Freiwilligenarbeit stellt ohne Zweifel einen wichtigen gesellschaftlichen Bereich dar, ohne jener zahlreiche Leistungen und Errungenschaften, sei es im Sozialbereich, in der Katastrophenhilfe, im Rettungswesen, im Sport, im Naturschutz etc., in der aktuellen Form nicht verfügbar und finanzierbar wären. Zunehmend richten sich die Anforderungen jedoch nicht nur an die durch die Freiwilligenarbeit bereitgestellten Leistungen, sondern betreffen auch die Freiwilligenarbeit selbst. Die Freiwilligenarbeit soll zusätzlich, d.h. als ein ihr immanentes Nebenprodukt, eine sozial integrative Wirkung auf Migrantinnen und Migranten, auf Arbeitslose und auf Pensionistinnen und Pensionisten entfalten. Sie soll ein Feld für den Erwerb sozialer und fachlicher Kompetenzen darstellen, sie soll die Gesundheit und die Lebenszufriedenheit fördern, sie soll demokratische Grundwerte vermitteln und generell das Vertrauen in die Gesellschaft erhalten und stärken, und das, idealerweise ohne dabei bezahlte Arbeitsplätze zu gefährden bzw. zu ersetzen. Die Fülle an Anforderungen und angenommenen Wirkungen wird nur selten vor dem Hintergrund der sozialen Nebenwirkungen der Freiwilligenarbeit diskutiert. Dabei ist der Zugang zu den Freiwilligenorganisationen seit je in hohem Ausmaß sozial selektiv und die interne Aufgaben- und Funktionsverteilung spiegelt vielfach die ungleichen Machtverhältnisse der Gesellschaft wieder. Bislang haben jedoch die empirischen Befunde zu den sozialen Determinanten der Freiwilligenarbeit weder Eingang in den politischen Diskurs gefunden, noch wurde der Freiwilligensektor von der Ungleichheitsforschung als relevantes Feld der Genese und Reproduktion sozialer Ungleichheit aufgegriffen. Die vorliegende Arbeit hat sich somit zum Ziel gesetzt, einen Beitrag zur Schließung dieser Lücke zwischen politischem Diskurs, empirischer Evidenzen und gesellschaftstheoretischer Betrachtung zu leisten. Dazu wird in einem ersten Schritt aus einer theoretischen Perspektive der Zusammenhang von Freiwilligenarbeit mit der Reproduktion sozialer Ungleichheit erörtert. In einem zweiten 
Schritt wird die aktuelle Forschung zu den Determinanten und den Auswirkungen der Freiwilligenarbeit aufgearbeitet. Anschließend werden in einem dritten Schritt empirische Daten zur Freiwilligenarbeit in Österreich analysiert.

Der Arbeit liegt dabei folgendes persönliches Forschungsparadigma zugrunde: Strukturbedingte Grenzen individueller Handlungsmöglichkeiten sollen mittels wissenschaftlicher Methoden aufgedeckt und bewusst gemacht werden, mit dem Ziel, diese zumindest potenziell gestalt- und veränderbar zu machen.

Es sei vorweg darauf hingewiesen, dass in der vorliegenden Arbeit die Begriffe, Freiwilligenarbeit, Ehrenamt und Freiwilliges Engagement synonym verwendet werden. Keiner der Begriffe deckt m.E. die Bedeutung des Phänomens freiwilliger, unbezahlter Tätigkeiten in gemeinnützigen Organisationen umfassend ab, sondern betont jeweils nur einen spezifischen Aspekt. Freiwilliges Engagement betont die individuelle, persönliche Dimension, den Input. Freiwilligenarbeit streicht die Tätigkeit selbst und deren Rahmenbedingungen hervor und grenzt sich so von Erwerbsarbeit, Familienarbeit und Zwangsarbeit ab. Ehrenamt wiederum stellt die symbolische Dimension der Anerkennung und des Prestiges ins Zentrum.

\section{Problemstellung}

Formelle Freiwilligenarbeit ${ }^{1}$, verstanden als freiwilliges und unbezahltes Engagement in gemeinnützigen Organisationen und Vereinen, ist in den letzten Jahren wiederholt hoch im Kurs, wenn es um Lösungsansätze gesellschaftspolitischer Problemlagen geht (vgl. Badelt/More-Hollerweger 2007; More-Hollerweger et al. 2009b). Der Freiwilligenarbeit wird vor allem Potential bei der Reintegration von Arbeitslosen (kritisch dazu Strauß 2009) und bei der Integration von Migrantinnen und Migranten in die (Aufnahme-)Gesellschaft zugesprochen (kritisch dazu Reinprecht 2009). Die negativen Effekte der zunehmenden gesellschaftlichen Überalterung (kritisch dazu Meyer et al. 2009) sollen durch Freiwilliges Engagement abgefedert werden und die ländliche Abwanderung und der Verlust an gesellschaftlichem Zusammenhalt gebremst werden. Darüber hinaus wird Freiwilliges Engagement auch als Mittel zur Demokratieförderung und als Ort lebenslangen Lernens konstruiert (vgl. European Volunteer Centre 2006; United Nations 2002). Gesamt betrachtet steht dahinter die Hoffnung, dass die Freiwilligenarbeit die zunehmend defizitärere wohlfahrtsstaatliche Daseinsversorgung sowie die generelle Ressourcenknappheit im Nonprofit Sektor zum Teil

1 in Abgrenzung zu informellen Formen der Freiwilligenarbeit wie z.B. Nachbarschaftshilfe (vgl. Badelt/More-Hollerweger 2007; More-Hollerweger et al. 2009b). 
kompensieren kann (vgl. Braun 2001a). Vor dem Hintergrund des empirischen Forschungstandes sind die von öffentlicher und politischer Seite geäußerten Erwartungen jedoch kritisch zu hinterfragen (vgl. Priller 2010, 2011). Die Kritik der vorliegenden Arbeit betrifft somit vor allem die verkürzte und verkürzende Perspektive auf (1) die integrativen und inklusiven Potentiale der Freiwilligenarbeit, (2) die dafür verantwortlich gemachten Wirkmechanismen, (3) die daraus abgeleiteten Effekte der Freiwilligenarbeit sowie (4) auf die Rolle der Freiwilligenarbeit bei der Reproduktion sozialer Ungleichheiten.

\section{(1) Überschätzung des integrativen Potentials der Freiwilligenarbeit}

Die Freiwilligenarbeit wird von österreichischen Politikern gerne als Mittel zur Integration von Migrantinnen und Migranten konstruiert.

„Österreich ist ein Land der Freiwilligkeit. Ehrenamt und Freiwilligkeit stärkt den sozialen Frieden und ist ein wesentlicher Grundpfeiler unseres Sozialsystems. Gerade für die Integration ist Freiwilligkeit ein wichtiger Faktor. Denn, wenn man sich engagiert in Vereinen und wenn man sich einer Gruppe zugehörig fühlt, dann funktioniert Integration. Daher ist es mir ein besonderes Anliegen, dass Migranten zur Freiwilligkeit motiviert werden“ („Mut zur Initiative“: der Staatssekretär Kurz über Ehrenamt und Eigeninitiative - Österreichisches Staatssekretariat für Integration $)^{2}$.

Die empirischen Befunde zeigen jedoch, dass nicht alleine die ethnische Herkunft entscheidet für den Zugang zur Freiwilligenarbeit ist, sondern der Bildungsgrad, das verfügbare Einkommen und die Vermögensausstattung, der Erwerbstatus, der Familienstand, die Anzahl der Kinder u.v.m. (vgl. Gensicke/ Geiss 2010; Rameder/More-Hollerweger 2009: 55ff; Stadelmann-Steffen et al. 2010; Statistik Austria 2007). Die Politik richtet damit den Blick eindimensional auf das soziale Merkmal der ethnischen Herkunft anstatt soziale und ökonomische Bedingungen zu schaffen, die Freiwilligenarbeit als Ausdruck einer freiwilligen, selbstgewählten und mit Sinn belegten Tätigkeit, ermöglichen. Denn bildungsferne Schichten, Erwerbslose, Personen über 65 Jahre, sowie die Bevölkerung in Großstädten weisen ähnlich niedrige Beteiligungsquoten auf wie Migrantinnen und Migranten.

Unter dem Slogan „Integration durch Leistung“ wird Freiwilligenarbeit nun zusätzlich auch als Indikator für gelungene Integration konstruiert und definiert ${ }^{3}$.

2 http://www.integration.at/news/news.aspx?nwo=35 - abgerufen am 7.1.2014. Das 2011 gegründete Staatssekretariat für Integration wurde im Zuge der Regierungsbildung im Herbst 2013 in das Bundesministerium für Europa, Integration und Äußeres integriert.

3 Staatssekretariat für Integration. 
Mitte 2013 erfolgte die entsprechende Änderung des Staatsbürgerschaftsgesetzes von $1985^{4}$. Fremde haben nun die Möglichkeit, ihre nachhaltige persönliche Integration nebst entsprechenden Sprachkenntnissen auch durch „a) ein mindestens dreijähriges freiwilliges, ehrenamtliches Engagement in einer gemeinnützigen Organisation, die den Vorgaben des $\$ 35$ Bundesabgabenordnung (BAO), BGBl. Nr. 195/1961, entspricht, oder [...] c) die Bekleidung einer Funktion in einem Interessenverband oder einer Interessenvertretung für mindestens drei Jahre hindurch" ${ }^{\text {, }}$, zu begründen und nachzuweisen.

„Darunter fallen klassisch Feuerwehr, Rotes Kreuz oder Samariter. Darüber hinaus sind laut Auskunft des Kurz-Büros Vereine umfasst, die die Integration in die österreichische Gesellschaft fördern und ,keine Segregationselemente` aufweisen. Ein Blasmusikverein wäre folglich eine Option, ein türkischer Kulturverein eher nicht" (Zwei Wege zur Einbürgerung im Expressverfahren, Die Presse, 5. Februar 2013).

Mit den Änderungen im Staatsbürgerschaftsgesetz wird Leitungsgerechtigkeit angestrebt, ohne jedoch im Vorfeld für Verteilungsgleichheit zu sorgen.

„Integration ist ein langfristiger und umfassender Prozess: Ziel ist es ,Integration durch Leistung möglich zu machen, das heißt, Menschen sollen nicht nach ihrer Herkunft, Sprache, Religion oder Kultur beurteilt werden, sondern danach, was sie in Österreich beitragen wollen. Dazu ist es wichtig, Leistung zu ermöglich, einzufordern und anzuerkennen, um eine umfassende Teilhabe an der Gesellschaft allen Bürgerinnen und Bürgern zu ermöglichen“ (Bundesministerium für Europa, Integration und Äußeres)

Somit wird auch in diesem Fall das individuelle „Wollen" vor das strukturelle „Können“" gestellt. Es wird zwar auch darauf hingewiesen, dass Leistung ermöglicht werden muss, was dafür nötig ist, wird jedoch nicht erwähnt. Ohne weiterer Maßnahmen ist zu erwarten, dass der Versuch den Einfluss von Herkunft, Sprache, Religion und Kultur durch Leistungsgerechtigkeit zu eliminieren dazu führt, dass Ungleichheit nur vordergründig reduziert wird, jedoch in Form ungleicher Beteiligungs- und Zugangschancen zur Freiwilligenarbeit weiterhin bestehen bleibt. Diesbezügliche empirische Befunde fehlen bis dato. Es ist bisher nicht öffentlich gemacht worden in wie vielen Fällen die gesetzliche Neuerung

4 Bundesgesetzblatt vom 30. Juli 2013 (BGBl. I Nr. 136/2013).

5 Staatsbürgerschaftsgesetz 1985, Fassung vom 28.01.2014 (http://www.ris.bka.gv.at/ GeltendeFassung.wxe?Abfrage=Bundesnormen \&Gesetzesnummer=10005579) - abgerufen am 28.5.2014.

6 http://www.bmeia.gv.at/aussenministerium/integration/?nwo=35 - abgerufen am 28.5.2014. 
bisher zur vorzeitigen Erlangungen der Staatsbürgerschaft geführt hat und welche Personengruppen davon konkret profitiert haben.

\section{(2) Romantisierung der Wirkmechanismen}

Neben den integrativen Potentialen selbst sind auch die Annahmen zu den Wirkmechanismen, d.h. zur Art und Weise wie die Freiwilligenarbeit konkret zur Integration beiträgt, eher fragwürdiger Natur: „Vereine sind der Ort, an dem Zusammenhalt gelebt wird und wo Integration ganz von alleine passiert" Dieser Form der sozial-romantischen Heuristik stehen zahlreiche Forschungsarbeiten der Sozialkapitalforschung ${ }^{8}$ zu den desintegrativen, unsozialen und negativen Effekten des Engagements in freiwilligen Vereinigungen entgegen (vgl. dazu u.a. Roth 2004; van Deth 2010). Beruhigend ist dabei lediglich, dass an diesen „Integrations-Automatismus“ in der Praxis selbst Funktionäre aus dem sonst so „integrationspotenten“ Sportbereich (kritisch dazu Nichols/Ralston 2011; Seippel 2005) nicht glauben: „Es liegt nicht nur bei den Migranten, dass sie auf uns zukommen, sondern wir müssen auch unseren Vereinen klarmachen, dass sie die Türen öffnen sollen “9. Es gilt daher in Zukunft soziale Integration durch Freiwilligenarbeit weder als Automatismus noch als unbeeinflussbare Blackbox dem Zufall zu überlassen. Von Seiten der Politik wie von den Freiwilligenorganisationen ist eine zielgerichtete Gestaltung der Rahmenbedingungen gefordert.

\section{(3) Heuristiken bezüglich der Effekte der Freiwilligenarbeit}

Von der arbeitsmarktpolitischen Konkurrenzdebatte hinsichtlich der Substitutionseffekte zwischen unbezahlten und bezahlten Mitarbeiterinnen und Mitarbeiter abgesehen (vgl. Haider/Schneider 2009), überwiegt in der politischen wie öffentlichen Diskussion die Betonung der positiven Auswirkungen der Freiwilligenarbeit. Dabei fußen die Erwartungen der Politik an den die Freiwilligenarbeit dokumentierenden Freiwilligenpass vorwiegend auf monokausalen Heuristiken und weniger auf evidenzbasierter Empirie. Auf der Webseite des

7 geäußert von Integrationsstaatssekretär Sebastian Kurz im Rahmen einer Pressekonferenz zum Projekt „Vereine machen auf, Migranten machen mit. Jetzt du! Dein Land braucht dich (Kurz will Migranten zur Freiwilligenarbeit motivieren, DerStandard, 11. Juni 2012).

8 Vergleiche dazu die Sozialkapital Forschung zu „brigding and bonding social capital“ (Coffé/Geys 2007).

9 Generalsekretär der Sportunion Österreich, Rainer Rösslhuber (DerStandard, 11. Juni 2012). 
österreichischen Bundesministeriums für Arbeit, Soziales und Konsumentenschutz (BMASK) wird „der Österreichischer Nachweis über Freiwilligenarbeit“ wie folgt beworben:

„Freiwilliges Engagement ist das Sozialkapital unserer Gesellschaft. Wesentlich ist jedoch, dass freiwilliges Engagement niemals hauptberufliche, bezahlte Erwerbsarbeit ersetzen kann und darf. Sie stellt eine zusätzliche Bereicherung für die soziale Qualität in unserem Land dar. Freiwilliges Engagement ist auch ein vielfach ungehobener Schatz für beruflichen Erfolg. Denn viele Fähigkeiten und Fertigkeiten, die in der Freiwilligenarbeit trainiert werden - sei es im Sozialbereich, in der Katastrophenhilfe oder im Umweltschutz - spielen in unserer Berufswelt eine wachsende Rolle“ (BMASK ${ }^{10}$ ).

In welcher Form und in welchem Ausmaß in der Freiwilligenarbeit Fähigkeiten, Fertigkeiten und Kompetenzen erworben werden und wie diese sich auf den beruflichen Erfolg auswirken, ist bislang kausalanalytisch nur in Ansätzen erforscht (siehe Kapitel (I).3.). Die vorhandenen Ergebnisse deuten jedenfalls darauf hin, dass vor allem bereits in den sozialen, kulturellen wie ökonomischen Dimensionen privilegierte Personen von der Freiwilligenarbeit profitieren. Es kann daher bei den Auswirkungen der Freiwilligenarbeit von einem sogenannten Matthäus-Effekt ${ }^{11}$ (vgl. Merton 2010; Rigney 2010) gesprochen werden.

„Denn ehrenamtliche Arbeit setzt in gewissem Umfang Human- und Sozialkapital sowie Reputation voraus. Durch die Ausübung eines Ehrenamtes werden diese Ressourcen aber weiter vergrößert, während Personen, die sich nicht ehrenamtlich betätigen, zum einen mit einer höheren Wahrscheinlichkeit von vornherein über weniger Ressourcen verfügen und zum anderen diese Unterschiede durch ihr „Nicht-Engagement“ noch vergrößert werden“ (Erlinghagen 2003: 754).

Erlinghagen (2003) geht davon aus, „dass von gemeinnützigem Engagement tendenziell auch das Risiko einer zunehmenden sozialen Spaltung ausgeht" (754) (vgl. auch Brömme/Strasser 2001). Darüber hinaus können auf individueller Ebene mit Freiwilligem Engagement auch direkte negative Effekte wie z.B.

10 http://www.bmask.gv.at/site/Soziales/Freiwilliges_Engagement/Freiwilliges_ Engagement_in_Oesterreich/Oesterreichischer_Nachweis_ueber_Freiwilligenarbeit - abgerufen am 28.5.2014.

11 Der Begriff „Matthäus-Effekt“ geht auf das Gleichnis von den anvertrauten Talenten im Matthäusevangelium zurück: „Denn wer hat, dem wird gegeben, und er wird im Überfluss haben; wer aber nicht hat, dem wird auch noch weggenommen, was er hat." (Mt 25,29). Im wissenschaftlichen Kontext hat Robert D. Merton (2010) den Begriff im Rahmen seiner Analyse der Zitierhäufigkeit von wissenschaftlichen Publikationen geprägt und für die Bezeichnung, der von ihm empirisch beobachteten positiven Rückkopplungseffekte (Erfolg führt zu Erfolg) verwendet. 
Burnout (vgl. Moreno-Jime'nez/Hidalgo Villodres 2010) verbunden sein. Auch hier stellt sich die Frage, ob alle sozialen Schichten in gleichem Ausmaß von den negativen Effekten betroffen sind und dadurch mögliche Wechselwirkungen zwischen Freiwilligenarbeit und gesamtgesellschaftlicher Ungleichheit bestehen.

\section{(4) Rolle der Freiwilligenarbeit bei der Reproduktion sozialer Ungleichheiten}

In den überwiegenden Fällen wird in der öffentlichen Diskussion und der medialen Berichterstattung implizit der Zusammenhang zwischen Freiwilligenarbeit und sozialer Ungleichheit in eine Richtung und auf zwei Wirkweisen postuliert: Freiwilligenarbeit bzw. Freiwilligenorganisationen reduzieren soziale Ungleichheit. Erstens, indem das Engagement für die freiwillig Tätigen selbst sozial integrierend und kompetenzerweiternd wirkt, und zweitens, überwiegend Menschen aus sozial benachteiligten Gruppen die Empfänger der unentgeltlich erbrachten, sozialen bzw. karitativen Leistungen sind. Die positiven Potentiale und Effekte von Freiwilligem Engagement scheinen dabei meist ohne empirische Belege als sakrosankt, wohingegen dessen soziale Bedingtheit und mögliche soziale Nebenwirkungen nur selten in den Blick genommen werden (vgl. Munsch 2010). Wie ein offizielles Dokument des Rats der Europäischen Union zum Europäischen Jahr der Freiwilligentätigkeit (2011) belegt, wurde die Geschlechterungleichheit in der Freiwilligenarbeit erkannt, die Ungleichheitsthematik im Gesamten bislang nicht zum Thema der Politik ${ }^{12}$ gemacht.

„Das ,Europäische Jahr der Freiwilligentätigkeit` zur Förderung der aktiven Bürgerschaft könnte [Hervorh. PR] auch dazu beitragen, geschlechterbezogene Ungleichheiten im Freiwilligensektor anzugehen, beispielsweise in Bezug auf die Sektoren und Bereiche, in denen Männer und Frauen tätig sind, oder in Bezug auf Repräsentation in ehrenamtlichen Führungspositionen“ (Der Rat der Europäischen Union 2010: L17/44).

In der Freiwilligenforschung gibt es jedoch bereits über die Genderthematik hinausreichende empirische Belege für die Reproduktion sozialer Ungleichheit in der Freiwilligenarbeit. Die ungleiche Vermögensverteilung führt dazu, dass Menschen, die sich kein Wohneigentum leisten können eine geringere soziale Verwurzelung am Wohnort (stake in society) aufweisen und infolge auch seltener

12 Weitere Dokumente zu den politischen Erwartungen an das freiwillige Engagement finden sich auf nationalstaatlicher (BMASK 2009; Deutschen Bundestag. EnqueteKommission „Zukunft des Bürgerschaftlichen Engagements“ 2002), europäischer (Der Rat der Europäischen Union 2010; European Volunteer Centre 2006) und internationaler Ebene (United Nations 2009). 
Freiwilligenarbeit leisten (vgl. Rotolo et al. 2010). Betreffend der intergenerationalen Reproduktion sozialer Ungleichheit zeigen aktuelle Ergebnisse, dass Aufgrund des inflationären Wertes höherer Bildungstitel zunehmend Freiwilliges Engagement eine kompensatorische Funktion bei der intergenerationalen Weitergabe von beruflichem Status übernimmt (vgl. van Houten et al. 2013). Freiwilligenarbeit bzw. Ehrenamt gewinnt damit scheinbar (wieder) an Bedeutung als Mittel zur Distinktion und Form der Weitergabe von sozialem und symbolischem Kapital (vgl. auch Ehrhardt 2011).

Zusammenfassend lässt sich daher festhalten, dass der Kompetenzerwerb in der Freiwilligenarbeit ins Zentrum gerückt, die sozialen Zugangsbarrieren hingegen ausgeblendet werden. Gerade jedoch die Verbindung des ungleichen Zugangs mit den positiven Effekten der Freiwilligenarbeit verweist auf deren ungleichheitsgenerierende und reproduzierende Wirkung. Auch Van Ingen (2009) sieht Freiwillige Vereinigungen weniger als egalitäre Organisationen, sondern vielmehr als Orte, an denen soziale Ungleichheit reproduziert wird. „Conversely, privileged citizens - who do not need the benefits of associational involvement in the first place - show the highest membership rates and occupy the most important positions within associations" (ebd.:144). Die Verantwortung für die verzerrte und einseitige Wahrnehmung der Potentiale, Mechanismen und Effekte der Freiwilligenarbeit gänzlich an die Politik abzugeben wäre einer kritischen Sozialforschung unwürdig. Es wird daher in einem nächsten Schritt der Blick auf mögliche Ursachen und Hintergründe geworfen.

\section{Hintergrund}

Als mögliche Hintergründe für die vernachlässigte Perspektive auf die Zusammenhänge von Freiwilligenarbeit und sozialer Ungleichheit lassen sich folgende vier Ursachen beschreiben: (1) der Fokus auf ökonomische Dimensionen sozialer Ungleichheit; (2) die oftmals vernachlässigte Heterogenität des Phänomens der Freiwilligenarbeit; (3) die Vernachlässigung der internen hierarchischen Strukturierung; sowie (4) die oftmals fehlende gesellschaftstheoretische Einbettung der empirischen Forschungsergebnisse.

\section{(1) Fokus auf ökonomische Dimensionen}

Der mediale wie gesellschaftspolitische Diskurs über gesellschaftliche Ungleichheit bezieht sich vorwiegend auf die ungleiche Verteilung von Vermögen und Einkommen (vgl. Pikkety 2014; Stiglitz 2012). Der Fokus auf die Entwicklung der Einkommens- und Vermögensungleichheit (z.B. Gini-Koeffizient) ist nicht zuletzt durch die Folgen der Finanzkrise nachvollziehbar. Die Ungleichheiten in 
den sozialen, kulturellen und symbolischen Dimensionen sind dadurch jedoch aus dem Blick geraten. So ist zu beobachten, dass trotz des in den letzten Jahren in der Politik und Forschung deutlich gestiegenen Interesses an der Freiwilligenarbeit diese bislang nur vereinzelt im Zusammenhang mit sozialer Ungleichheit untersucht worden ist.

\section{(2) Vernachlässigte Heterogenität}

Freiwilligenarbeit findet in funktional-differenzierten Gesellschaften nicht im kontext- und regelfreien Raum statt, sondern ist in unterschiedliche gesellschaftliche Felder (Sport, Politik, Sport, Bildung, Religion, Soziales, Gesundheit, etc.) eingebettet, die wiederum jeweils durch eigene Zugangs- und Strukturierungsregelungen gekennzeichnet sind (vgl. Bourdieu 1982, 1992b). Der medial wie öffentlich geführte Diskurs über die Freiwilligenarbeit beschränkt sich jedoch vielfach auf die sozialen Engagementformen wie z.B. jene im Rahmen der Sozialen Dienste (z.B. Caritas, Volkshilfe, etc.) der Rettungs- und Katastrophenhilfe (z.B. Rotes Kreuz, Samariterbund, etc.) sowie des Aktivismus für Menschenrechte (z.B. Amnestie International, etc.) und Umweltthemen (z.B. Greenpeace, Vier Pfoten, etc.). Damit geraten jene Bereiche, Felder und Organisationen der Freiwilligenarbeit aus dem Blick, die z.B. explizit oder implizit überwiegend den Eigeninteressen und eigenen Bedürfnisse der Freiwilligen bzw. dem erweiterten u.a. ebenfalls sozial selektierten Mitgliederkreises (z.B. exklusive Clubs) dienen. Im groben lassen sich dabei binnenorientierte und außenorientierte Vereine und Organisationen unterscheiden (vgl. Braun 2007). Erstere erstellen Leistungen primär für ihre Mitglieder, wie dies in Sportvereinen, Autofahrerclubs, etc. der Fall ist. Zweitere erstellen Leistungen überwiegend für Personen bzw. Ziele außerhalb der Organisation, wie dies üblicherweise auf die meisten karitativen und sozialen Organisationen sowie auf Menschenrechtsorganisationen und Umweltschutzorganisationen zutrifft. So werden unter Freiwilligenarbeit eben nicht „nur" wohltätige und karitative Tätigkeiten verstanden, sondern der Definition folgend, alle unbezahlten, freiwilligen Tätigkeiten in Vereinen aller Art, vom Sport, über die Kultur, den Natur- und Tierschutz, politischer Aktivitäten, Interessensvertretungen, sowie zwar legale, jedoch antisozialen und demokratiegefährdende Gruppierungen (vgl. More-Hollerweger et al. 2009b). Aktuelle Studien belegen, dass auch die Tätigkeiten in bzw. die Ziele von Freiwilligenorganisationen selbst mit „unsozialen Effekten“ verbunden sein können, und diese nicht automatisch auf die Bekämpfung und Reduktion sozialer Ungleichheit ausgelegt sind. Diese reichen von negativen und demokratiegefährdenden Aspekten, wie dies zum Beispiel bei rechtsradikalen, populistischen oder 
autoritären Vereinigungen der Fall ist (vgl. Roth 2004; Ruzza 2009). Darüber hinaus hat sich in Studien ebenfalls bestätigt, dass sich Vereinsmitgliedschaften und Freiwilligenarbeit auch negativ auf die allgemeine Toleranz gegenüber anderen Bevölkerungsgruppen auswirken können und nicht automatisch gesellschaftliche Solidarität fördern (vgl. Iglic 2010; van Deth 2010).

Gesamtbetrachtet werden differenziertere Analysen zu den bereichsspezifischen Zugangsvoraussetzungen (Ressourcen bzw. Kapitalien) meist zugunsten verkürzender Gesamtaussagen (vgl. Braun 2003b, 2007; Vogt 2005) vernachlässigt. Braun (2003b) kritisiert diesbezüglich die im Diskurs und in empirischen Analysen oftmals fehlende Differenzierung zwischen der Vielzahl an freiwilligen Vereinigungen. Für ihn haben, aufgrund deren normativer Besetzung, gängige Aussagen zu Mitgliedschaftsquoten, gesellschaftlicher Integration und Gemeinwohlorientierung „eher metaphysischen als empirischen Charakter“ (101). So finden sich die Forschungslücken vor allem hinsichtlich der feldspezifischen Mechanismen und (Macht-) Strukturen, die zu sozial ungleichem Zugang zur Freiwilligenarbeit führen. Dabei variiert die Anzahl und Qualität der empirischen Arbeiten zwischen den einzelnen Engagementfeldern stark. Eine vergleichsweise lange Tradition hat die Forschung zum Freiwilligen Engagement in den Feldern der Politik (vgl. Verba et al. 1995; Weber 1921/1980), des Sports (vgl. Baur/Braun 2003; Baur et al. 2003; Braun 2003c; Nagel 2003b, c; Radtke 2007), der Sozialen Arbeit (vgl. Heimgartner 2004), der Kirche und der Religion (vgl. Bourdieu 1998: 186ff; Schell 2009) sowie Berufsverbänden (vgl. Nesbit/ Gazley 2012).

\section{(3) Vernachlässigung der internen Strukturierung}

Der Freiwilligensektor besteht aus unterschiedlichen, historisch geprägten Feldern und Engagementformen. So sind z.B. ehrenamtliche Führungspositionen in der Verwaltung von Städten in ihrer Entwicklung und sozialen Bedingtheit von der Freiwilligenarbeit in Vereinen, im Sinne von freiwilligen, selbstorganisierten Zusammenschlüssen zu trennen (vgl. Ehrhardt 2011: 59). Infolge dessen stellt sich die Frage nach dem sozial ungleichen Zugang zu den unterschiedlichen Funktionsbereichen und hierarchischen Positionen in der Freiwilligenarbeit und danach, ob im Freiwilligen Engagement nicht dadurch auch „klassische Rollen- und Verhaltensmuster eher noch verstärkt" werden (Zimmer 2009: 84). Eine differenzierte Erfassung sowie vergleichende Analyse des sozial ungleichen Zugangs nach Positionen und Status der freiwilligen Tätigkeiten hat bislang nur in vergleichsweise geringem Ausmaß, und interessanterweise im Gegensatz zur aggregierten Analyse des allgemeinen Zugangs, meist nur für einzelne Bereiche 
der Freiwilligenarbeit stattgefunden (Nagel 2003a; Radtke 2007; Schell 2009). Bis auf wenige Ausnahmen ${ }^{13}$, ist dies u.a. auch der empirischen Forschungstradition, d.h. konkret der forschungsökonomischen Integration von Erhebungsinstrumenten in bestehende Erhebungen ${ }^{14}$, und damit einer nur in wenige Dimensionen differenzierten Erfassung der Freiwilligenarbeit, geschuldet. Die interne Strukturierung und Hierarchisierung in der Freiwilligenarbeit ist im Kontext der Reproduktion sozialer Ungleichheit bislang eher stiefmütterlich betrachtet worden. Ehrhardt (2011) meint dazu in Bezug auf die Besetzung von Führungs- bzw. Elitepositionen im Ehrenamt,

„[d]ie >Kritik am Ehrenamt< beschränkt sich ausschließlich auf die Frage, ob hiermit auch anspruchsvolle Führungsaufgaben adäquat bewältigt werden können; der Selektionsmechanismus dagegen wird meist nicht wahrgenommen oder zumindest nicht öffentlich thematisiert" (Ehrhardt 2011: 61f).

\section{(4) Fehlende gesellschaftstheoretische Einbettung und Integration der Forschungsergebnisse}

Aus einer gesellschaftskritischen sowie theoretischen Perspektive wurde bislang die Frage, ob und in welcher Form aus dem sozial ungleichen Zugang (Heitzmann et al. 2009; Institute for Volunteering Research 2004) nicht nur Heterogenität, sondern tatsächlich auch soziale Ungleichheit im engeren Sinne resultiert, d.h. mit dem Zugang auch Vor- und Nachteile in anderen Lebensbereichen verbunden sind (Solga et al. 2009), nicht explizit und umfassend beantwortet. Bisher ist es in den meisten empirischen wie theoretischen Arbeiten bei einem Hinweis auf eine mögliche ungleichheitsreproduzierende bzw. eine sozial spaltende Wirkung der Freiwilligenarbeit geblieben (z.B. Erlinghagen 2003). Ein Beispiel dafür stellt der bislang umfassendste Literaturüberblick zur vorwiegend nordamerikanisch fundierten empirischen Freiwilligenforschung von Musick/Wilson (2008) dar. Betreffend des ungleichen Zugangs zur Freiwilligenarbeit schreiben sie, „there is no evidence to suggest any change in the class bias of volunteering or in the relative exclusion of racial and ethnic minority groups from voluntary work" (Musick/Wilson 2008: 533). Trotz dieser Conclusio sucht man im Index ihres 663 Seiten umfassenden Werkes, „Volunteers - A Social Profil“, vergeblich nach Begriffen wie „social inequality“ oder „social exclusion“ (ebd.). Ein möglicher

13 Deutschland: Freiwilligensurvey; Schweiz: Freiwilligenmonitor; Niederlande: Giving in the Netherlands Panel Survey (GINPS).

14 Z.B. European Value Survey (EVS), Survey of Health, Ageing and Retirement in Europe (SHARE), Sozio-ökonomische Panel (SOEP). 
Erklärungsversuch lautet folgendermaßen: Das in Abbildung 1 dargestellte Prozessmodell dient vielen Forschungsarbeiten als strukturierende Hintergrundfolie (vgl. Musick/Wilson 2008; Snyder/Omoto 2008; Wilson 2012). Der Nachteil des „Volunteer Process Model“ liegt m.E. darin, dass die Zusammenhänge zwischen den Prozessphasen (Antecedents - Experiences - Consequences) sowie den Analyseebenen (Individual - Interpersonal/Social Group - Agency/Organization - Societal/Cultural Context) tendenziell ausgeblendet werden. Die für eine (gesellschafts-)kritische Betrachtung bedeutsamen Wechselwirkungen sind möglicherweise dadurch vielfach unbeachtet geblieben.

Abbildung 1: Schematische Darstellung des Volunteer Process Model

\begin{tabular}{|l|l|l|l|}
\hline \multirow{2}{*}{$\begin{array}{l}\text { Level of } \\
\text { Analysis }\end{array}$} & \multicolumn{3}{|c|}{ Stages of the Volunteer Process } \\
\cline { 2 - 4 } & Antecedents & \multicolumn{1}{|c|}{ Experiences } & \multicolumn{1}{c|}{ Consequences } \\
\hline Individual & $\begin{array}{l}\text { Personality, motivation, } \\
\text { life circumstances }\end{array}$ & $\begin{array}{l}\text { Satisfaction, stigma, } \\
\text { organizational integration }\end{array}$ & $\begin{array}{l}\text { Knowledge and attitude } \\
\text { change, health }\end{array}$ \\
\hline $\begin{array}{l}\text { Interpersonal/ } \\
\text { Social Group }\end{array}$ & $\begin{array}{l}\text { Group memberships, } \\
\text { norms }\end{array}$ & $\begin{array}{l}\text { Helping relationship, } \\
\text { collective esteem }\end{array}$ & $\begin{array}{l}\text { Composition of social } \\
\text { network, relationship } \\
\text { development }\end{array}$ \\
\hline $\begin{array}{l}\text { Agency/ } \\
\text { Organization }\end{array}$ & $\begin{array}{l}\text { Recruitment strategies, } \\
\text { training }\end{array}$ & $\begin{array}{l}\text { Organizational culture, } \\
\text { volunteer placement }\end{array}$ & $\begin{array}{l}\text { Volunteer retention, work } \\
\text { evaluation }\end{array}$ \\
\hline $\begin{array}{l}\text { Societal/Cultural } \\
\text { Context }\end{array}$ & $\begin{array}{l}\text { Ideology, service } \\
\text { programs and institutions }\end{array}$ & $\begin{array}{l}\text { Service provision, } \\
\text { program development }\end{array}$ & $\begin{array}{l}\text { Social capital, economic } \\
\text { savings }\end{array}$ \\
\hline
\end{tabular}

Quelle: Snyder/Omoto (2008: 7).

Generell werden die Kausalitäten und Wechselwirkungen, wie sie bei der Reproduktion sozialer Ungleichheiten von Interesse sind, mit gängigen psychologischen und handlungsorientierten Prozessmodellen nur ungenügend oder gar nicht abgebildet. Die nötige Integration der unterschiedlichen Forschungsstränge zu den Determinanten sowie zu den Auswirkungen der Freiwilligenarbeit ist bislang ausgeblieben (vgl. Hustinx et al. 2010a; Snyder/Omoto 2008; Wilson 2012). Fragen zu den Zusammenhängen zwischen den sozio-ökonomischen Determinanten des Zugangs und den positiven wie negativen Effekten der Freiwilligenarbeit sind somit bisher vielfach unbeantwortet geblieben. Gesamt gesehen fehlt es den existierenden empirischen Ergebnissen zum sozial ungleichen $\mathrm{Zu}$ gang oftmals an deren gesellschaftstheoretischer Einbettung. In Folge hat die Forschung in diesem Bereich bislang nur wenig zur weiteren Theorieintegration 
bzw. Theoriebildung im Rahmen der Ungleichheitsforschung beitragen bzw. angeregt.

Zusammengefasst legt der öffentliche wie politische Diskurs zur Freiwilligenarbeit den Schluss nahe, dass der freie Zugang aller Gesellschaftsmitglieder zur Freiwilligenarbeit vorausgesetzt und Freiwilliges Engagement als multifunktionales Mittel zur sozialen Integration von Migranten und Migrantinnen, zur Reintegration von Arbeitslosen und anderen marginalisierten Gruppen sowie letztlich zur Demokratieförderung und als Maßnahme zum lebenslangen Lernen gesehen bzw. konstruiert wird. Vor diesem Hintergrund der weiterhin niedrigen Engagementquoten sozialstrukturell benachteiligter Bevölkerungsgruppen erscheinen die Ansprüche an die Freiwilligenarbeit mehr als überzogen. Will man Freiwilligenarbeit daher ernsthaft den wachsenden sozialen Ungleichheiten (vgl. Alber/Lenarz 2007; Goebel et al. 2007; Pikkety 2014; Solga et al. 2009; Stiglitz 2012; Therborn 2006) und gesellschaftlichen Herausforderungen, wie z.B. steigender Arbeitslosigkeit, Migration, Überalterung und Budgetknappheit entgegenstellen, sollte diese zuerst selbst umfassend auf deren eigene, implizite wie explizite Beiträge zur (Re)Produktion sozialer Ungleichheit untersucht werden. Dabei ist der ungleiche Zugang nicht nur gesellschaftspolitisch von Bedeutung, sondern gewinnt auch zunehmend für das Management von Nonprofit Organisationen an Relevanz. Dies betrifft die Koordination von Freiwilligen, die Rekrutierung, die Definition und Verteilung von Aufgaben und Positionen, Fragen der Diversität sowie Fragen der internen wie externen Governance, des Social Impact Assessments und der Corporate Social Responsibility im Allgemeinen.

Die identifizierten Forschungslücken betreffen somit einerseits den ungleichen Zugang zur Freiwilligenarbeit selbst, d.h. zu den Feldern und Positionen, als auch die theoriefundierte Verbindung der Forschungsergebnisse zum ungleichen Zugang und den damit verbundenen individuellen, institutionellen bzw. organisationalen wie gesamtgesellschaftlichen Konsequenzen. Daraus abgeleitet lautet die Forschungsfrage:

In welcher Form und in welchen Bereichen leistet die Freiwilligenarbeit einen Beitrag zur Genese und Reproduktion sozialer Ungleichheit?

\section{Aufbau der Arbeit}

Die Arbeit ist in vier zentrale Kapitel gegliedert. Die ersten zwei Kapitel widmen sich den unterschiedlichen theoretischen Ansätze und Perspektiven (Kapitel I) sowie dem Stand der Forschung zu den Determinanten und Auswirkungen der Freiwilligenarbeit (Kapitel II). Daran anschließend folgt die eigene empirische Analyse und die Interpretation der Ergebnisse (Kapitel III). Abschließend 
werden im letzten Kapitel die Schlussfolgerungen und daraus abgeleitete Empfehlungen für die weitere Forschung, die Politik und die Managementpraxis formuliert (Kapitel IV).

Ungleichheitstheoretische Ansätze sowie die Verortung der Freiwilligenarbeit innerhalb dieser Ansätze stehen im Fokus des ersten Kapitels. Dazu folgt im ersten Unterkapitel ein kurzer Abriss zu den Definitionen, Formen wie Dimensionen sozialer Ungleichheit. Anschließend werden im zweiten und dritten Unterkapitel zwei aktuelle theoretische Perspektiven der Ungleichheitsforschung vorgestellt und der Versuch unternommen, die Freiwilligenarbeit in diesen Modellen kausal mit der Genese und Reproduktion sozialer Ungleichheit in Beziehung zu setzen. Im vierten Unterkapitel liefert Pierre Bourdieus Theorie der Praxis Erklärungsansätze dafür, warum ein Bereich wie die Freiwilligenarbeit, trotz des Fehlens direkter ökonomischer Profite für die Akteure, den sozialen Kämpfen um symbolische wie soziale Profite unterliegt. Sie liefert darüber hinaus den theoretischen Rahmen die unterschiedlichen feldspezifischen Bedingungen der Freiwilligenarbeit in den Blick zu nehmen. Dazu werden in einem ersten Schritt die zentralen Theoreme Bourdieus vorgestellt und anschließend anekdotische Bezüge Bourdieus zur Freiwilligenarbeit diskutiert. Im fünften Unterkapitel werden die erarbeiteten theoretischen Perspektiven in einem Modell der (Re-)Produktion sozialer Ungleichheit in der Freiwilligenarbeit zusammengeführt.

Im zweiten Kapitel wird in einem ersten Schritt das Thema der sozialen Ungleichheit innerhalb der zentralen Forschungsstränge der Freiwilligenforschung verortet. In einem zweiten Schritt folgt die Darstellung der empirischen Befunde zu den Determinanten der Freiwilligenarbeit, konkret zu den askriptiven und erworbenen sozialen Merkmalen. Die empirischen Erkenntnisse zu den Auswirkungen der Freiwilligenarbeit, d.h. zu den Effekten, die die Freiwilligen selbst betreffen, werden dann im dritten Unterkapitel zum Stand der Forschung diskutiert.

Das dritte Kapitel widmet sich schließlich der Analyse von Sekundärdaten zur Freiwilligenarbeit in Österreich. Einer kurzen Erläuterung zu den formulierten Fragestellungen folgt eine Darstellung zur Erhebungsmethodik und zur Verortung des verwendeten Sekundärdatensatzes im Kontext der Engagementforschung. Daran anschließend werden die angewandten statistischen Analyseverfahren beschrieben. Die empirischen Ergebnisse werden in vier Unterkapiteln dargestellt und interpretiert. Einer bivariaten vergleichenden Analyse zur Sozialstruktur in Österreich und der Freiwilligenarbeit folgt die multivariate Analyse zum Einfluss der zentralen sozialen Merkmale auf den Zugang zur Freiwilligenarbeit wie deren Einfluss auf die interne hierarchische Strukturierung. Die Analysen werden anhand von vier ausgewählter Felder weiter differenziert und 
vertieft. Abschließend wird diskutiert, welche Erkenntnisse die empirischen Ergebnissen zur Erklärung des Beitrags der Freiwilligenarbeit zur Reproduktion sozialer Ungleichheit liefern und wo die Grenzen der vorliegenden Analysen sind.

Im vierten und letzten Kapitel werden die Schlussfolgerungen der Arbeit vor dem Hintergrund ihrer Relevanz für die Praxis, d.h. für politische Entscheidungsträger und für die Freiwilligenorganisationen diskutiert sowie der damit verbundene zukünftige Forschungsbedarf erörtert. 



\title{
(I) Theoretische Perspektiven auf soziale Ungleichheit und Freiwilligenarbeit
}

\begin{abstract}
„Die soziale Welt vergibt das seltenste Gut überhaupt: Anerkennung, Ansehen, das heißt ganz einfach Daseinsberechtigung [...] Weniges ist so ungleich und wohl nichts grausamer verteilt als das symbolische Kapital, das heißt die soziale Bedeutung und die Lebensberechtigung" (Bourdieu 2001b: 309f).
\end{abstract}

Die Rolle der Freiwilligenarbeit bei der Entstehung und der Reproduktion sozialer Ungleichheit ist bislang kaum erforscht worden und infolge bislang auch nicht Ziel sozialpolitischer Interventionen geworden. Dies ist kaum verwunderlich, denn im öffentlichen wie medialen Diskurs wird primär die Einkommensungleichheit (z.B. Gini-Koeffizient) bzw. die Ungleichverteilung von Vermögen thematisiert (vgl. Pikkety 2014; Stiglitz 2012). Ökonomisches Kapital stellt jedoch nur eine von mehreren Dimensionen sozialer Ungleichheit dar (vgl. Hradil; Kreckel 2004; Solga et al. 2009). Neben dem Bildungsgrad und den mit Einkommen oftmals parallel laufenden Dimensionen wie Macht und Prestige sind, zumindest in den westlich orientierten Ländern, neue Dimensionen wie Freizeit,- Wohn- und Arbeitsbedingungen ungleichheitsrelevant geworden (vgl. Hradil 2005: 485). Je nach theoretischem Blickwinkel werden unterschiedliche Gesellschaftssysteme für die Ungleichheiten verantwortlich gemacht. Ganz vorn stehen meist das Bildungssystem, das Wirtschaftssystem sowie der Rechtsstaat, der für die Umverteilung von Vermögen zuständig ist (vgl. Rehberg 2011). Die Zivilgesellschaft und der Dritte Sektor ${ }^{15}$ werden zwar aus der Ungleichheitsdiskussion nicht explizit ausgeklammert, die Vielzahl an Freiwilligen Vereinigungen - alleine in Österreich gibt es derzeit über 116.000 Vereine - finden in der Ungleichheitsforschung jedoch keine oder nur eine randständige Beachtung. Vor dem Hintergrund differenztheoretischer (vgl. Schwinn 2007) als auch feldtheoretischer Überlegungen (vgl. Bourdieu 1982, 1992a) sind jedoch alle

15 „Die Gesamtheit der NPOs wird auch häufig als Dritter Sektor bezeichnet und damit den Sektoren »Markt« und »Staat« gegenübergestellt" (Meyer/Simsa 2013: 9). 
Institutionen und Organisationen mit ihren differenzierenden und strukturierenden Prozessen und Hierarchien soziale Kampfplätze und damit potentielle Ort der Genese und Reproduktion sozialer Ungleichheit (vgl. Diewald/Faist 2011). Die nachfolgenden Kapitel widmen sich aus einer theoretischen Perspektive der Frage, wie Freiwilligenarbeit (als Tätigkeit, als Position, als Feld, etc.) konzeptionell in die bestehenden Erklärungsmodelle bzw. -ansätze der Entstehung und Reproduktion sozialer Ungleichheit anschlussfähig gemacht und integriert werden kann.

\section{(I) 1. Soziale Ungleichheit - Definitionen, Formen und Dimensionen}

Fragen zur Ausprägung und den Ursachen sozialer Ungleichheit zählen von Beginn an (vgl. Marx/Engels 1977 (1848); Weber 1921/1980) zu den zentralen Themen der Soziologie (vgl. Berger 2004; Budowski/Nollert 2010; Hradil 2005; Hradil 2008; Kreckel 2004; Solga et al. 2009). Entsprechend zahlreich sind die Arbeiten und Ansätze, die sich mit Fragen der ungleichen Ressourcenverteilung in Gesellschaften beschäftigen. Die Annahme gottgewollter und damit natürlicher gesellschaftlicher Unterschiede ist ausgehend von Jean-Jaques Rousseau heute nicht mehr haltbar (vgl. Berger 2004). Es gilt heute aus einer wissenschaftlichen Perspektive als unbestritten, „dass soziale Ungleichheit gesellschaftlich produziert und damit auch gesellschaftlich gestalt- bzw. veränderbar" ist (Solga et al. 2009: 11). Das Adjektiv sozial bezieht sich dabei auf den Entstehungsprozess der Ungleichheit, d.h. Soziale Ungleichheiten bezeichnen Ungleichheiten, die durch menschliche Interaktion und durch menschliches Handeln hergestellt und reproduziert werden. Trotz intensiver Auseinandersetzung, in theoretischer wie empirischer Form, gibt es bis dato keine "Grand Theory" bzw. nicht die Grundlagentheorie zur Erklärung sozialer Ungleichheit (vgl. Berger 2004; Diewald/Faist 2011: 93; Rehberg 2011: 17). Überwiegend Einigkeit herrscht in der Ungleichheitsforschung hinsichtlich der zentralen Definitionsmerkmale sozialer Ungleichheit sowie betreffend ihrer vielfältigen Erscheinungsformen, wie in den Dimensionen Einkommen, Macht, Prestige aber auch Gesundheit, der Lebenserwartungen sowie der gesellschaftlichen Teilhabe.

\section{(I) 1.1. Definitionen sozialer Ungleichheit}

Gemein ist den meisten Definitionen, dass unter sozialer Ungleichheit, der an soziale Merkmale gebundene, systematisch und dauerhaft ungleiche Zugang zu mit ungleichen Lebenschancen verbundenen sozialen Positionen verstanden wird (vgl. 
Hradil 2009:286; Kreckel 2004: 17; Solga et al. 2009: 15). Die folgenden drei Definitionen sozialer Ungleichheit geben dazu einen Einblick: Solga et al. (2009) sprechen

„immer dann von sozialer Ungleichheit, wenn Menschen (immer verstanden als Zugehöriger sozialer Kategorien) einen ungleichen Zugang zu sozialen Positionen haben und diese sozialen Positionen systematisch mit vorteilhaften oder nachteiligen Handlungs- und Lebensbedingungen verbunden sind. [...] Es handelt sich also um überindividuelle Ungleichheiten in der Verteilung von Handlungsressourcen sozialer Gruppen, die durch das Verhalten und Denken des Einzelnen nicht kurzfristig beeinflusst werden können. [...] Diese Definition von sozialer Ungleichheit enthält keine Vorentscheidung darüber, wie gerecht oder ungerecht, und damit, wie legitim soziale Ungleichheit ist. Dies ist eine normative Frage, deren Antwort bei den einzelnen Forscherinnen und Forschern liegt" (15).

Für Kreckel (2004) liegen

„Soziale Ungleichheit im weiteren Sinne [...] überall dort vor, wo die Möglichkeiten des Zugangs zu allgemein verfügbaren und erstrebenswerten sozialen Gütern und/oder zu sozialen Positionen, die mit ungleichen Macht und/oder Interaktionsmöglichkeiten ausgestattet sind, dauerhafte Einschränkungen erfahren und dadurch die Lebenschancen der betroffenen Individuen, Gruppen oder Gesellschaften beeinträchtigt bzw. begünstigt werden [...] Anders ausgedrückt, bei den objektiven Ungleichheiten, die der Soziologe ermittelt, handelt es sich um Handlungsbedingungen, die Handlungsspielräume eröffnen oder auch begrenzen (17ff).

\section{Ähnliches formuliert Hradil (1987) im Rahmen seines Lagen und Milieu Konzepts:}

„Unter sozialer Ungleichheit sind gesellschaftlich hervorgebrachte und relativ dauerhafte Handlungsbedingungen zu verstehen, die bestimmten Gesellschaftsmitgliedern die Befriedigung allgemein akzeptierter Lebensziele besser als anderen erlauben [...] Sowohl die angesprochenen Lebensziele wie auch die Handlungsbedingungen sind Produkte menschlichen Handelns und nicht etwa anonyme Verursachungsprozesse“ (Hradil 2009:286).

Im Zentrum der Ungleichheitsforschung steht somit der ungleiche Zugang zu sozialen Positionen und/oder sozialen Gütern, jedoch nur insofern, als mit dem ungleichen Zugang zu diesen Positionen bzw. Gütern auch Vor- bzw. Nachteile in anderen (Lebens-)Bereichen verbunden sind. Verschiedenartigkeiten, Heterogenitäten und Prozesse sozialer Differenzierung sind zwar die Ausgangspunkte für soziale Ungleichheit jedoch nur dann relevant, wenn sie mit sozial strukturierten, relativen dauerhaften Vor- oder Nachteilen für Angehörige bestimmter Merkmalsgruppen verbunden sind (vgl. Kreckel 2004; Solga et al. 2009). 
„In Abgrenzung zu sozialer Ungleichheit bezeichnen wir als soziale Differenzierung gesellschaftlich verankerte (also gleichfalls überindividuelle) Unterschiede, die nicht (notwendigerweise) mit Vor- und Nachteilen und somit mit Asymmetrien in den Handlungsbedingungen verbunden sind"(Solga et al. 2009: 15).

\section{(I) 1.2. Formen sozialer Ungleichheit}

Je nach Ansatz werden in der Literatur unterschiedliche Formen sozialer Ungleichheit unterschieden. Eine geläufige Unterscheidung ist jene in Chancenungleichheit und Ergebnis- bzw. Verteilungsungleichheit (vgl. Solga et al. 2009: 21). Als Chancenungleichheit werden von Solga et al. (2009) nur die auf zugeschriebene Merkmale, d.h. Geschlecht, Alter, Ethnie beruhenden, „ungleiche[n] Chancen von sozialen Gruppen beim Zugang zu sozialen Positionen oder Handlungsressourcen (zum Beispiel zu Bildungs-, Arbeitsmarkt- oder Einkommenspositionen)“ verstanden (21). Demgegenüber bezeichnen Ergebnis- bzw. Verteilungsungleichheit die „Vor- und Nachteile, die sich durch den Besitz wertvoller Güter oder durch den Zugang zu erstrebenswerten Positionen ergeben (zum Beispiel ungleiche Einkommen, Arbeitsbedingungen, Lebensstandards, etc.)“ (ebd.: 21f.).

Kreckel (2004) unterscheidet zwei Aggregatszustände strukturierter sozialer Ungleichheit. Der erste Aggregatszustand umfasst den ungleichen Zugang „zu allgemein verfügbaren und erstrebenswerten soziale Gütern" und wird von Kreckel als sozial strukturierte Verteilungsungleichheit bezeichnet. Als zweiten Aggregatszustands nennt Kreckel die sozial strukturierte Beziehungsungleichheit und meint damit die Ausstattung von sozialen „(erworbenen oder zugeschriebenen) Positionen mit ungleichen Handlungs- und/oder Interaktionsbefugnissen oder -möglichkeiten“ (Kreckel 2004: 19f).

„Sowohl ungleich verteilte Güter als auch asymmetrische Beziehungen werden als strategische Ressourcen aufgefaßt. [...]. Sind diese Ressourcen ungleich verteilt, so haben wir mit »objektiven « Ungleichheiten zu tun. D.h., wer privilegierten Zugang zu diesen Ressourcen erlangt, gewinnt damit für die Verwirklichung seiner speziellen Wünsche, Bedürfnisse, Lebensentwürfe usw. günstigere objektive Bedingungen als derjenige, der von diesen Ressourcen ausgeschlossen bleibt“ (vgl. Kreckel 2004: 20).

In diesem Kontext weisen Diewald/Faist (2011) unter Bezug auf Amartya Sen (1999) darauf hin, dass es zu bedenken gilt, „dass Beteiligungschancen nicht für alle Gesellschaftsmitglieder die gleiche Bedeutung haben müssen“ und damit konsequent von Verwirklichungschancen zu unterscheiden sind (98). 


\section{(I) 1.3. Dimensionen sozialer Ungleichheit}

Hradil erklärt pointiert, was mit Dimensionen sozialer Ungleichheit gemeint ist:

„Die Vielfalt vorhandener sozialer Ungleichheiten wird in der Regel in Dimensionen gebündelt. In modernen Gesellschaften gelten der formale Bildungsgrad, die mehr oder minder sichere Erwerbstätigkeit, die berufliche Stellung, das Einkommen bzw. Vermögen und das berufliche Prestige als wichtigste Dimensionen sozialer Ungleichheit. [...] Innerhalb jeder dieser Dimensionen lassen sich höhere oder niedrigere Stellungen unterscheiden. Sie werden als Bildungs-, Erwerbs-, Berufs-, Einkommens- bzw. PrestigeStatus bezeichnet" (Hradil 2013: 154).

Die Erscheinungsformen und damit die Dimensionen sozialer Ungleichheit sind nicht absolut zu denken sondern verschieben und verändern sich mit dem fortlaufenden gesellschaftlichen Wandel.

„So war formale Bildung noch im ausgehenden Mittelalter für die Mehrzahl der Menschen eher unwichtig. Heute spricht viel dafür, dass der erreichte Bildungsgrad für die Menschen die wichtigste Dimension sozialer Ungleichheit darstellt (Hradil 2013: 154).

Eine etwas abstraktere und offenere Klassifikation vertritt Kreckel (2004) im Rahmen seiner „politischen Soziologie der sozialen Ungleichheit“. Er unterscheidet vier Dimensionen strukturierter sozialer Ungleichheit (siehe Abbildung 2) und bezeichnet diese aus Akteursperspektive auch als strategische Ressourcen: Materieller Reichtum (1) und symbolisches Wissen (2) als distributive Ungleichheiten, sowie hierarchische Organisation (3), und selektive Assoziation (4) als relationale Ungleichheiten (vgl. Kreckel 2004: 75ff). Dabei wird deutlich, dass Kreckel die vier Dimensionen sozialer Ungleichheit den gleichen vier Sphären zuordnet, in denen auch Bourdieu (1983) seine vier grundlegenden Kapitalarten, das ökonomische, das kulturelle, das soziale und das symbolische Kapital verortet.

\section{Abbildung 2: Dimensionen strukturierter sozialer Ungleichheit}

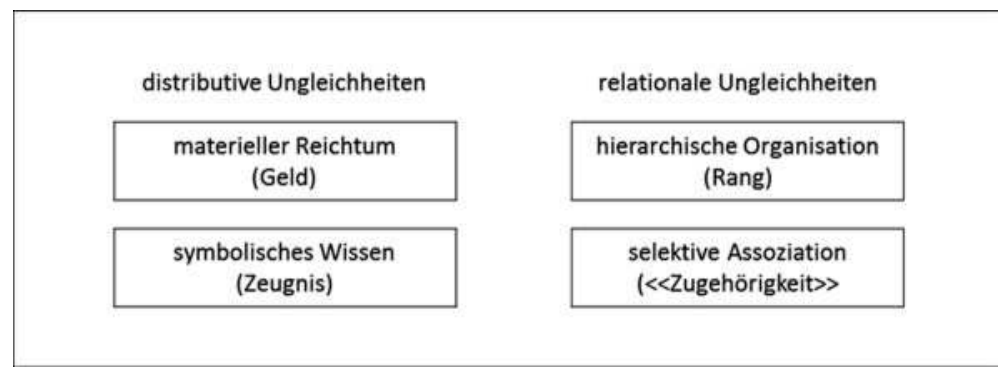

Quelle: in Anlehnung an Kreckel (2004: 94). 
1. Von der Dimension des materiellen Reichtums spricht Kreckel (2004) immer dann, wenn

„der Zugang zu (primär) materiellen Produkten und Bedingungen vergangener und/ oder gegenwärtiger menschlicher Arbeit in asymmetrischer Weise reguliert ist und dadurch ungleiche Handlungsbedingungen und Lebenschancen entstehen" (78).

Empirisch stellt sich damit die Frage, „welche knappen materiellen Güter in einer Gesellschaft in der Weise zugangsreguliert sind, daß sie sich als ungleichheitskonstituierende Handlungsbedingungen auswirken“ (ebd.). Mit knappen materiellen Gütern, sind in markt- und geldwirtschaftlich orientierten Gesellschaften primär Güter gemeint, „die von privater Seite käuflich erworben werden können“ (ebd.).

2. Neben dem materiellen Reichtum zählt die Dimension des (symbolischen) Wissens zum Bereich der distributiven Ungleichheit. Darunter fasst Kreckel (2004) „das kulturelle Erbe einer Gesellschaft, ihre Sprache, Sitten, Techniken, Gebräuche usw." als Gemeingüter zusammen und betont, dass auch „symbolische Güter zugangsreguliert und ungleich verteilt“ sein können, jedoch deren Knappheit, im Gegensatz zu materiellen Gütern, schwerer vorstellbar sei (79). Als Beispiele für systematische Verknappung nennt Kreckel u.a. die Monopolisierung religiöser wie juristischer Einsichten, den eingeschränkten Zugang zu Bildungsinstitutionen wie den damit verbundenen Qualifikationen und Zeugnissen, oder aber auch die Benachteiligung von sprachlichen Minoritäten (z.B. Dialekt versus Hochsprache) (vgl. ebd.).

„Es geht dabei um die ungleich verteilte Fähigkeit, die »geistigen Objektivationen« bzw. das verfügbare kulturelle Erbe entschlüsseln, anwenden, für sich zu beanspruchen bzw. vorteilhaft nutzen zu können“" (Kreckel 2004: 79)

3. In den Bereich der relationalen Ungleichheit fällt die Dimension der hierarchischen Organisation.

„Sie kann Mittel zum Zweck, sie kann aber auch Selbstzweck sein. So mag eine Führungsposition in Politik oder Wirtschaft z.B. persönliche Eitelkeiten und Machtgelüste befriedigen, und sie kann auch Möglichkeiten zur Durchsetzung sinnvoller Ideen oder zur Leistung brauchbarer Arbeit bieten“" (Kreckel 2004: 81f).

Kreckel (2004) streicht dabei heraus, dass die Lebenschancen wie die tatsächliche Lebensführung von den Positionen innerhalb von (hierarchischen) Organisationen mitbestimmt werden (vgl. 81f).

"Gleichgültig, in welcher Hierarchie man eine Position innehat - sei es nun in einem Betrieb, einer Schule, einem Verein - je höher die Stellung, desto größer sind (ceteris 
paribus) die Möglichkeiten, über andere Menschen und deren Handeln verfügen zu können. Höhere Position verleiht größere Handlungsautonomie und beeinflußt damit auch Lebenschancen“ (Kreckel 2004: 82).

Damit ist Kreckel einer der wenigen im Feld der Ungleichheitsforschung, der die Positionsbesetzung in Vereinen und damit innerhalb der formellen Freiwilligenarbeit explizit benennt. In der empirischen Forschungspraxis findet dies jedoch bislang kaum eine Entsprechung.

4. Die vierte Dimension strukturierter sozialer Ungleichheiten wird als jene der selektiven Assoziation definiert. Diese beruht für Kreckel (2004) „,auf symmetrische Beziehungen zwischen Gleichen, einander als gleichartig und/oder gleichwertig Anerkennenden" (83). Eine Intensivierung dieser exklusiv gestalteten Beziehungen kann „zu einer Verstärkung und Verfestigung des allgemeinen Zustandes der Ungleichheit beitragen" (83). Selektive Assoziationen umfassen nach Kreckel (2004) „Familien- und Verwandtschaftsverbindungen oder landsmannschaftliche Loyalitäten [...] Freundschaften, studentische Verbindungen, berufsständische Gemeinschaften, politische Loyalitäten, gemeinsame Verkehrskreise“ (84). Letztlich sind damit alle Verbindungen, Verpflichtungen und erworbenen Gemeinsamkeiten gemeint, die im Sinne Bourdieus (1983) Sozialkapitalfunktion erfüllen und damit „als Startbasis für den Zugang zu anderen Ressourcen und damit zur Monopolisierung von privilegierten Lebenschancen" nutzbar sind (84). Kreckel (2004) merkt dazu weiter an:

„Soziale Ungleichheit aufgrund von selektiver Assoziation läßt sich, im Gegensatz zu den in den drei anderen Dimensionen, in unserer heutigen Gesellschaft nicht mehr ohne weiteres offen legitimieren. Soziale Vorteile und Vorurteile aufgrund von Familienbindungen oder Freundschaften und Bekanntschaften, ebenso wie von Rasse und Geschlecht verstoßen gegen die offiziell proklamierten und auch verfassungsmäßig verankerten Grundwerte der Chancengleichheit und Leistungsgerechtigkeit“ (Kreckel 2004: 85).

Kreckel (2004) wie auch Bourdieu (1977) vertreten die These, dass soziale Ungleichheit bzw. die herrschende Ordnung von den beteiligten und betroffenen Akteuren selbst meist nicht als solche wahrgenommen und hinterfragt wird.

„Soziale Ungleichheit ist eine von Menschen gemachte und damit von Menschen veränderbare Grundtatsache heutigen gesellschaftlichen Lebens. Von den Beteiligten und Betroffenen wird sie allerdings häufig als unabänderliches Schicksal hingenommen. Dennoch besteht die Hoffnung, daß mit der genaueren Einsicht in die Entstehungsund Wirkungsweise sozialer Ungleichheitsverhältnisse auch deren vermeintlichen Selbstverständlichkeit und Unantastbarkeit ins Wanken gebracht werden kann“ (Kreckel 2004: 13). 
Bourdieu $(1977,1998)$ bezeichnet dieses Phänomen innerhalb seiner Theorie als Doxa. „Doxa, das ist der Glaube, der sich als solcher nicht kennt“ (FuchsHeinritz/König 2005:201). „Jede herrschende Ordnung weist die Tendenz auf - allerdings auf unterschiedlicher Stufe und mit je anderen Mitteln -, ihren spezifischen Willkürcharakter zu naturalisieren" (Bourdieu 1977: 324). Gerade im Kontext der Freiwilligenarbeit spielt dieser Aspekt eine besondere Rolle. Der Begriff der Freiwilligenarbeit, der zwar noch nicht vollständig jedoch in vielen Bereichen den Begriff des Ehrenamts abgelöst hat, trägt zusätzlich zur Verschleierung sozial bedingter, strukturierter, dauerhafter Benachteiligungen bei. Durch das Attribut der Freiwilligkeit scheint sich dieser Bereich aktuell mehr oder weniger erfolgreich einer umfassenderen Sozialkritik entzogen zu haben.

Um sich der Fragestellung nach dem Zusammenhang von sozialer Ungleichheit und Freiwilligenarbeit auf theoretischer wie empirischer Ebene weiter nähern zu können, haben sich für meine Arbeit zwei theoretische Perspektiven bzw. Ansätze als hilfreich herausgestellt. Erstens die Unterscheidung von vier Strukturebenen sozialer Ungleichheit, wie sie Solga et al. (2009) vorschlagen, sowie der Forschungsansatz von Diewald/Faist (2009), der den Prozess von Heterogenitäten hin zu sozialen Ungleichheiten nachzuzeichnen versucht. Aus der ersten Perspektive stellt sich die Frage, ob und wenn ja, auf welchen der Strukturebenen sozialer Ungleichheit die Freiwilligenarbeit verortet werden, und welche Schlüsse dadurch über das mögliche Zusammenwirken von Freiwilligenarbeit und sozialer Ungleichheit getroffen werden können. Die zweite Perspektive lenkt den Blick auf die Freiwilligenarbeit als Kontext der Entstehung und Reproduktion sozialer Ungleichheit. In einem nächsten Schritt werden die beiden Ansätze erklärt und daran anschließend mit der Freiwilligenarbeit in Verbindung gebracht.

\section{(I)2. Theoretische Perspektiven zu den Struktur- und Kontextebenen sozialer Ungleichheit}

\section{(I) 2.1 Strukturebenen sozialer Ungleichheit}

Solga et al. (2009) unterscheiden in ihrem Prozessmodell (siehe Abbildung 3) vier Strukturebenen sozialer Ungleichheit (vgl. Hradil 2008: 213ff; Solga et al. 2009: 16ff). Zentral dabei ist, dass es sich um eine Frage der kausalanalytischen Interpunktion handelt (vgl. Solga et al. 2009), ob eine Ressource bzw. eine Kapitalform Ausgangspunkt oder Endpunkt sozialer Ungleichheit darstellt. Auf der Inputseite stehen die sogenannten (1) Determinanten sozialer Ungleichheit, die durch (2) näher zu bestimmende Mechanismen auf der Outputseite zu sozialer 
Ungleichheit in unterschiedlichen (3) Dimensionen führen, die ihrerseits wieder mit bestimmten (4) Auswirkungen verbunden sind. Damit haben Solga et al. (2009) ein theoretisches Modell vorgestellt, das die Notwenigkeit unterstreicht, bei der Erforschung sozialer Ungleichheit mit kausalanalytischen Interpunktionen zu arbeiten.

Abbildung 3: Strukturebenen sozialer Ungleichheit

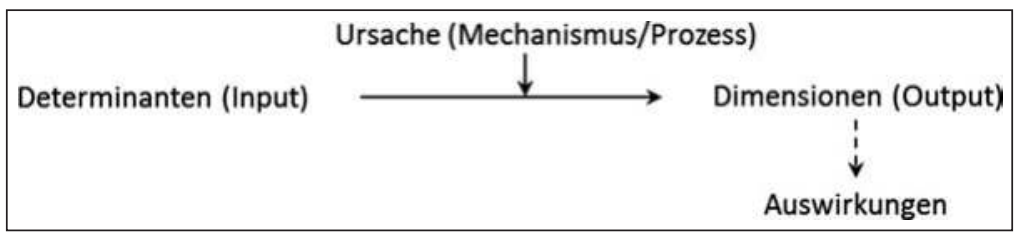

Quelle: Solga et al. (2009: 17).

Die Determinanten sozialer Ungleichheit bezeichnen „soziale Merkmale von Personen (wie zum Beispiel das Geschlecht, das Bildungsniveau, die soziale Herkunft), die Zugehörigkeiten zu sozialen Gruppen definieren“ (Solga et al. 2009: 16). Die Zugehörigkeit zu diesen sozialen Gruppen ist „wiederum Grundlage für Vor- und Nachteile in bestimmten Handlungs- und Lebensbedingungen" (ebd.). Dabei muss zwischen zugeschriebenen Merkmalen (Geschlecht, Alter, soziale und regionale Herkunft) und erworbenen Merkmalen (Beruf, Bildungsgrad, Familienstand) unterschieden werden (vgl. ebd.). Zentral ist, dass diese Determinanten soziale Positionen kennzeichnen, „die an sich keine Besseroder Schlechterstellung darstellen, aber diese mit hoher Wahrscheinlichkeit nach sich ziehen“ (Hradil 2005: 34f). So sind z.B. mit einem bestimmten Beruf, Geschlecht oder Körpergewicht grundlegend keine sozialen Vor- und Nachteile verbunden. Erst die jeweiligen gesellschaftlichen Verhältnisse, die Bewertung sowie soziales Handeln selbst führen zu einer sozial vermittelten Statuszuweisung eines Berufs, zu einer Schlechterstellung von Frauen oder aber einer negativen Bewertung und strukturellen Diskriminierung von Übergewichtigen, d.h. dazu, dass aus Verschiedenartigkeit soziale Ungleichheit resultiert (vgl. Solga et al. 2009: 16).

Unter den Dimensionen sozialer Ungleichheit verstehen Solga et al. (2009) die wichtigsten Arten von Vor- und Nachteilen (vgl. ebd.: 18). Als Basisdimensionen sozialer Ungleichheit gelten „materieller Wohlstand“, „Macht“ und „Prestige“ (Hradil 2005: 31). Die drei Basisdimensionen wurden schließlich im Zuge postindustrieller gesellschaftlicher Veränderungen um die Dimension der „Bildung“ 
erweitert. Laut Hradil sind jedoch auch diese vier Dimensionen in modernen Gesellschaften nicht mehr ausreichend. Neben Zielvorstellungen wie Gesundheit treten zunehmend auch Ressourcen und Handlungsmöglichkeiten in den Blick und „lassen es notwendig erscheinen, auch auf soziale Ungleichheiten innerhalb der Dimensionen Arbeits-, Wohn-, Umwelt- und Freizeitbedingungen einzugehen" (ebd.: 31).

Von besonderem Interesse sind die Ursachen, d.h. die konkreten sozialen Mechanismen und Prozesse, die dazu führen, dass aus Unterschieden (z.B. Geschlecht) soziale Ungleichheit (Diskriminierung beim Zugang zu Führungspositionen) werden. Denn ...

„Ursachen sozialer Ungleichheit sind die sozialen Prozesse oder sozialen Mechanismen, durch die die Zugehörigkeit zu bestimmten Sozialkategorien in einer Art und Weise sozial relevant wird, dass diese zu Vor- und Nachteilen in anderen Lebensbereichen (Dimensionen) führt“ (Solga et al. 2009: 19).

Solga et al. (2009) nehmen selbst keine eigene Klassifikation der bekannten Mechanismen sozialer Ungleichheit vor, sondern fordern vielmehr von einer zeitgemäßen empirischen Forschung ein, konkrete Mechanismen zu spezifizieren und zu benennen.

Die „Auswirkungen sozialer Ungleichheit stellen schließlich die Konsequenzen der sozial strukturierten Vor- und Nachteile dar" (Solga et al. 2009: 20). Schlussendlich weisen Solga et al. (2009) darauf hin, dass es von der kausallogischen sowie theoretischen Perspektive des Forschers bzw. der Forscherin abhängt, ob es sich bei einem Phänomen um eine Dimension oder eine Auswirkung sozialer Ungleichheit handelt (ebd.).

Nachfolgend sollen zwei konkrete Bespiele das Prozessmodell der Strukturebenen sozialer Ungleichheit illustrieren: Beispiel (1): Das Geschlecht (Determinante) führt u.a. über den Mechanismus der sogenannten Statistischen Diskriminierung ${ }^{16}$ (Ursache) dazu, dass Frauen seltener in Führungspositionen (Dimension) zu finden sind und dadurch geringere Einkommen (Auswirkung) beziehen. Beispiel (2): Die Höhe des Bildungsgrads (Determinante) führt über

16 „Von statistischer Diskriminierung wird [...] allgemein dann gesprochen, wenn Entscheidungen über das einzelne Individuum auf Grundlage von Verhaltensannahmen bezüglich ganzer sozialer Gruppen getroffen werden“ (vgl. Solga et al. 2009: 20). Zum Beispiel, die Annahme von Arbeitgebern, dass Frauen aufgrund von Haushalts- und Kinderbetreuungspflichten zeitlich weniger flexibel sind als Männer. Dies wird unterstellt, ohne tatsächlich über die Lebenssituation der einzelnen Frau Bescheid zu wissen (vgl. ebd.). 
die „Kopplung des Zugangs zu Arbeitsplätzen an Bildungsabschlüsse“ (Ursache) zu ungleichen Einkommen (Dimension), die wiederum zu ungleichen Armuts- und Gesundheitsrisiken (Auswirkungen) führen. Der Nutzen dieses Modells liegt darin, dass es einfordert, genau zu definieren „Zwischen welchen Determinanten und Dimensionen ein Zusammenhang besteht" und, dass der konkrete Mechanismus bezeichnet und erklärt werden muss, „durch den soziale Ungleichheit hergestellt wird“" (ebd.: 20f). Die Aufnahme der Auswirkungen in das Prozessmodell hat im Rahmen der vorliegenden Forschungsarbeit anfänglich eher für Verwirrung als für Klarheit gesorgt. Schlussendlich hat die Auseinandersetzung damit bewirkt, den Stand der Forschung zu den Auswirkungen der Freiwilligenarbeit im Allgemeinen, und davon abgeleitet möglichen Auswirkungen eines ungleichen Zugangs zur Freiwilligenarbeit im Besonderen genauer in den Blick zu nehmen. Somit ist das Prozessmodell wie es Solga et al. (2009) vertreten geeignet, die mögliche Rolle und Bedeutung vom ungleichen Zugang zur Freiwilligenarbeit theoretisch zu erörtern, ein Strukturierungsschema für den Stand der Forschung bereitzustellen sowie bestehende Forschungslücken offen zu legen. D.h. konkret der Frage nach zu gehen, ob der empirisch belegte ungleiche Zugang zur Freiwilligenarbeit lediglich eine Form sozialer Differenzierung darstellt und Ausdruck von Verschiedenartigkeit ist, oder aber damit auch soziale Ungleichheit verbunden sein kann.

\section{(I) 2.2. Kontextebenen sozialer Ungleichheit}

Für Diewald/Faist (2011) ist ebenfalls die Frage zentral, wie aus Heterogenitäten soziale Ungleichheiten werden und welche Mechanismen dafür verantwortlich sind. Damit unterscheidet sich ihr Ansatz nicht grundlegend von jenem der vier Strukturebenen, jedoch stellen sie nicht wie Solga et al. (2009) ein Strukturierungsschema für Theorien sozialer Ungleichheit bereit, sondern wollen damit selbst ein neues Forschungsprogramm ${ }^{17}$ der Ungleichheitsanalyse fundieren (vgl. SFB 882).

„Im spezifischen Feld der Ungleichheitsforschung propagieren wir diesbezüglich, konsequent Heterogenitäten als Ausgangspunkt und Ungleichheiten als Endpunkt der Mechanismen zu benennen, also nicht zu konfundieren und nebeneinanderzustellen.

17 z.B. der von der Deutschen Forschungsgemeinschaft (DFG) geförderte Sonderforschungsbereich (SFB 882): Von Heterogenitäten zu Ungleichheiten (http://www. sfb882.uni-bielefeld.de/ - abgerufen am 28.5.2014). 
Dabei gilt es, Heterogenitätsmerkmale und Ungleichheiten jeweils im Plural zu konzipieren, um der Multidimensionalität und den möglichen Interdependenzen angemessen Rechnung tragen zu können“" (Diewald/Faist 2011: 94).

Abbildung 4 stellt diesen theoretischen Ansatz dar. Diewald/Faist (2011) fassen unter Heterogenitäten alles zusammen, „was die Unterschiedlichkeit und Vielfalt von Individuen ausmacht" (95). Sie unterscheiden unter Rückgriff auf bestehende Einteilungen vier Gruppen von Merkmalen. Diese umfassen jegliche Form von (1) askriptiven Merkmalen, darüber hinaus jedoch (2) kulturelle Lebensstile, Einstellungen, Vorlieben etc., (3) Kompetenzen, Qualifikationen und Eigenschaften sowie (4) Tätigkeiten im Rahmen gesellschaftlicher Arbeitsteilung wie in der Erwerbsarbeit, der Hausarbeit und der Kinderbetreuung. Damit haben sie im Vergleich zu Solga et al. (2009) bzw. zu Hradil (2005) ein breiteres Verständnis von ungleichheitsrelevanten Merkmalen. Sie argumentieren diesbezüglich folgendermaßen: „Angesichts der Zunahme von Heterogenitäten steigen die Anforderungen an eine angemessene theoretische und empirischen Erfassung derjenigen Merkmale, die zum Ausgangspunkt von Ungleichheiten werden können" (ebd.: 97). Dabei betonen sie, dass die Unterscheidung nach Heterogenitätsmerkmalen erst durch „klassifikatorische Kämpfe und Verhandlungen verschiedener Akteure" in sozialen Feldern erzeugt wird ${ }^{18}$.

Analog zu Solga et al. (2009) betonen sie die Bedeutung sozialer Mechanismen bei der Erklärung sozialer Ungleichheiten. Mit ihrem Erklärungsprogramm wollen sich Diewald/Faist (2011) von Ansätzen abgrenzen, die folgende Merkmale aufweisen:

- „die Verpflichtung auf eine alles erklärende, rein philosophierende Grand Theory;

- die streng deduktive Erklärung über allgemeingültige Gesetze im engen Sinn, die die konkreten Wirkungsketten zwischen Ursache und Folge als „black box" behandeln und deshalb wenig zu ihrem Verständnis beitragen;

18 Diewald/Faist (2011) verweisen diesbezüglich auf die Arbeiten von Wimmer (2008), Barlösius (2005), sowie betreffend der sozialen Konstruktionen von Kategorien auf Butler (1991). Der hier m.E. naheliegende Rückgriff auf Bourdieus Habitus-KapitalFeld Konzept erfolgt nur indirekt über die Arbeiten von Barlösius (2005). 
Abbildung 4: Von Heterogenitäten zu Ungleichheiten

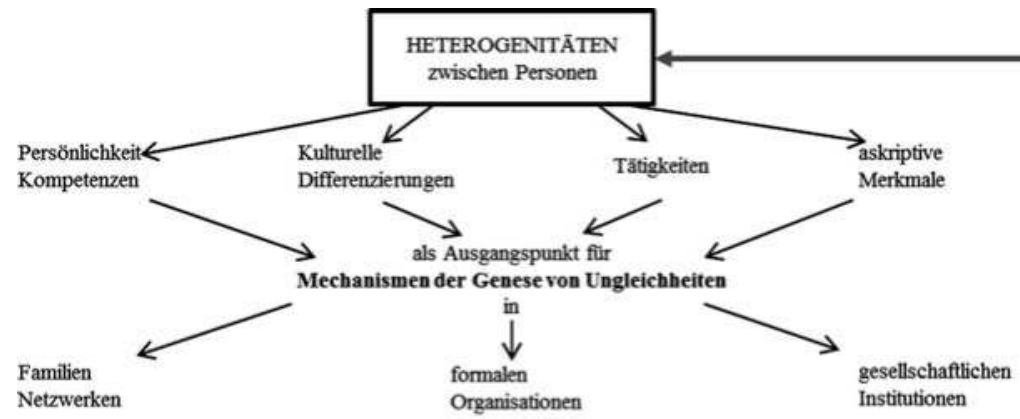

(1) Wahrnehmung und Bewertung von Heterogenitäten

Vorurteile

Stereotypisienung.

Thematisierung

Stereotypisienung,

Thematisienung

(2) Soziale SchlieBung: Inklusion/Exklusion

Zugehörigkeit

Mitgliedschaft

MigrantInnen-Rechte

(3) Soziale Schließung: Chancenhortung

Cliquen

Austauschnormen
Karrieremuster

Diversity Management

(4) Hierarchisierung

Netzwerk-, Familienrollen
Positionsstrukturen
Entlohnungssysteme
Klientelismus

Umverteilungsprinzipien

(5) Ausbeutung/Asymetrische Dependenz

Geldverteilung

Beschāftigungsverhaltnisse

strukturelle Gewalt

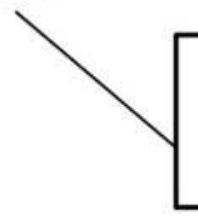

Interdependenzen: Generalisierung.

Konflikt, Konkurrenz,

Substitution

Unabhăngigkeit

über die Zeit: Kumulation vs. Wendepunkt

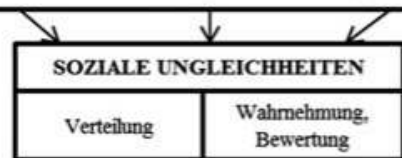

Quelle: Diewald/Faist (2011: 104). 
- ein Empirismus, der Gesetzmäßigkeiten trotz häufiger Probleme von Scheinkorrelationen, Endogenität und konfundierenden Variablen primär aus Korrelationen zwischen Variablen ableiten bzw. bestätigen will“" (Diewald/Faist 2011: 99).

Sie beziehen sich dabei auf die Arbeiten und Überlegungen zum Erklärungspotential von sozialen Mechanismen ${ }^{19}$ von Machamer et al. (2000), Elster (2007), Gross (2009) sowie Reskin (2003). Mangels einheitlicher Definition schließen sie sich folgender Grundidee sozialer Mechanismen an:

- „Die Identifizierung von Mechanismen ist nicht an eine bestimmte Theorie oder bestimmte methodische Herangehensweise gebunden.

- Mechanismen beziehen sich auf generative Prozesse, die unter bestimmten Ausgangsbedingungen bestimmte Ergebnisse hervorbringen. [...] Insofern Mechanismen unter den gleichen Bedingungen zuverlässig die gleichen Ergebnisse hervorbringen, sind sie regelhaft. Der Grad ihrer Verallgemeinerbarkeit oder gar universalen Geltung über über diese Regelhaftigkeit unter bestimmten Kontextbedingungen hinaus ist hingegen offen (z.B. Elster 2007). Dies unterscheidet sie von Gesetzmäßigkeiten im engeren Sinn“(Diewald/Faist 2011: 100).

Die Identifizierung von konkreten Mechanismen soll dazu beitragen, (soziale) Gesetzmäßigkeiten nicht rein empiristisch von nur auf Scheinkorrelationen beruhenden Zusammenhängen zwischen Variablen abzuleiten. Im Speziellen geht es Diewald/Faist (2011) bei der Anwendung eines sozialmechanismischen Ansatzes darum,

„1. empirisch konkrete, direkt beobachtbare, kontextspezifische und damit für die politische Beeinflussung relevante soziale Mechanismen der Genese und Wirkung sozialer Ungleichheiten zu identifizieren (konkret-substanzielle Mechanismen) und

2. längerfristig auf dieser Basis über Vergleiche und Abstrahierungen zu einer über verschiedene Gesellschaftsbereiche hinweg verallgemeinerungsfähigen , generative social grammar of inequality' (Therborn 2006, S. 1) zu kommen“" (Diewald/Faist 2011: 101).

Diewald/Faist (2011) benennen vier abstrakter Hauptmechanismen (siehe Abbildung 4): Prozesse der a) Exklusion/Inklusion (soziale Schließung) und b) Chancenhortung (soziale Schließung), c) Hierarchisierung sowie d) Ausbeutung bzw. asymmetrische Dependenz. Sie beziehen sich dabei auf die wenigen Arbeiten zu diesem Thema, u.a. auf die Klassiker von

19 Sozialmechanismische Ansätze haben in den letzten Jahren in den Sozialwissenschaften und insbesondere in der Ungleichheitsforschung an Bedeutung gewonnen (vgl. u.a. Reskin 2003; Therborn 2006; DiPrete und Eirich 2006). 
- Max Weber und Karl Marx zu sozialer Schließung und Ausbeutung,

- Charles Tilly (1998) zu Ausbeutung und Chancenhortung und

- Therborn (2006) zu den Mechanismen von Distantiation, Exclusion, Hierarchization, Exploitation ${ }^{20}$.

Wie in Abbildung 4 dargestellt, stellen sie diesen vier Hauptmechanismen noch eine fünfte Gruppe von Mechanismen voran, und zwar „solche der Wahrnehmung und Bewertung von Heterogenitäten. Diese Mechanismen betreffen noch nicht direkt die Genese sozialer Ungleichheiten im eigentlichen Sinne, sind aber diesen vorangeschaltet" (Diewald/Faist 2011: 105). Mechanismen der Exklusion bzw. Inklusion regeln den Zugang zu Netzwerken, Organisationen oder ganzen Gesellschaften. Bei Mechanismen der Chancenhortung geht es „dann um die Behandlung innerhalb dieser sozialen Bereiche“. Ausbeutung meint die „Vorteilnahme im Rahmen von Kooperationen“. Hierarchisierung bezeichnet die „Existenz von unterschiedlichen mit Rechten, Pflichten und Ressourcen ausgestatteten Positionen in formalen Organisationen [... sowie ...] informellen Rollensystemen und kulturellen Rangordnungen“ (Diewald/Faist 2011: 104f). Die Mechanismen führen in der Folge dazu, dass es zu einer Ungleichverteilung der klassischen Ressourcen wie Macht, Prestige und Geld kommt, aber auch zu ungleichen "Beteiligungschancen in unterschiedlichen Lebensbereichen" (Diewald/ Faist 2011: 98). Dabei verorten Diewald/Faist (2011) die sozialen Mechanismen auf drei unterschiedlichen Kontextebenen, (1) auf Ebene der Familie und Netzwerke, (2) auf Ebene der Organisationen und (3) auf Ebene gesellschaftlicher Institutionen:

„Diese Unterscheidung folgt der Logik, das es die Zuordnung von Personen mit bestimmten Merkmalen (Heterogenitäten) zu konkreten Positionen und Mitgliedschaften in informellen und institutionellen Ordnungen ist, die insgesamt der Ausprägung von sozialen Ungleichheiten zugrunde liegt“ (Diewald/Faist 2011: 105).

Eine weitere Annahme hinter dieser Unterscheidung ist auch, dass auf diesen drei Kontextebenen soziale Ungleichheiten nach unterschiedlichen Regeln wie

20 „Mit ,Distantiation` sind die Regeln des Wettbewerbs gemeint, die Gewinner und Verlierer mit bestimmten Abständen zwischen ihnen produzieren (z. B. MatthäusPrinzip); mit ,Exclusion' (inklusive Chancenhortung und sozialer Schließung) die Trennung in Insider und Outsider und die damit einhergehenden Diskriminierungen und Stigmatisierungen; mit ,Hierarchization' das Gefüge institutionalisierter Rollen und Positionen, die mit unterschiedlichen Rechten und Ressourcen verknüpft sind, und mit ,Exploitation' ein asymmetrischer Nutzen, den eine Seite aus einer Kooperation ziehen kann" (Diewald/Faist 2011: 102f) (siehe Anhang). 
auch in unterschiedlichen Dimensionen hergestellt werden (vgl. ebd.). In diesem Zusammenhang stellt die Erwerbsarbeit jedoch weiterhin den zentralen Ausgangspunkt für soziale Ungleichheiten dar (vgl. Kreckel 2004) d.h. „Positionen in hierarchisch strukturierten Positionssystemen der Erwerbsarbeit definieren wesentlich den Zugang zu den klassischen Ungleichheiten hinsichtlich Macht, Autonomie, Prestige und Einkommen“ (Diewald/Faist 2011: 98).

Für eine umfassendere Betrachtung ist es Diewald/Faist (2011) zufolge unabdingbar, dass auch andere Lebensbereiche, die mit für das Wohlbefinden relevanten Bedürfnissen im Zusammenhang stehen, in die Analyse sozialer Ungleichheiten mitaufgenommen werden (ebd.). Als Beispiele nennen sie,

„die Integration in soziale Beziehungen, die soziale und politische Partizipation sowie als spezieller Aspekt die Kombination von multiplen Zugehörigkeiten über nationale Gesellschaften hinweg" (Diewald/Faist 2011: 98).

Darüber hinaus ist auch das Zusammenspiel der unterschiedlichen Kontextebenen zu untersuchen, z.B.

„wenn etwa informelle Netzwerke direkt oder indirekt über das Homophilieprinzip Einstellungs- und Beförderungschancen in Betrieben bestimmen (Marsden und Gorman 2001; Elliott 2001)“(Diewald/Faist 2011: 105f).

Dabei gilt es den Blick vor allem darauf zu richten, in welchem Ausmaß und in welcher Form sich Mechanismen und in Folge die sozialen Ungleichheiten „in verschiedenen Handlungsfeldern wechselseitig eher ausgleichen oder verstärken“, d.h. es dadurch zu einer Kumulation von Chancen kommt oder soziale Schließung induziert und fortgeführt wird (ebd.:106). Die Wechselwirkungen zwischen den verschiedenen Mechanismen - sogenannte cumulative (dis)advantages - können Diewald/Faist (2011: 106) folgend in unterschiedlichen Formen auftreten.

\section{Abbildung 5: Formen kumulativer Vor- und Nachteile}

\begin{tabular}{c|c}
$\begin{array}{c}\text { sich gleichende Ungleichbehandlungen } \\
\text { in verschiedenen Lebensbereichen bzw. } \\
\text { Kontexten (z. B. Erwerbsarbeit und } \\
\text { Haushalt) }\end{array}$ & $\begin{array}{c}\text { wiederkehrende Ungleichbehandlungen } \\
\text { in aufeinanderfolgenden Lebensphasen } \\
\text { bzw. institutionell verknüpften Kontexten } \\
\text { (z. B. Ausbildung, Beruf) }\end{array}$ \\
\hline $\begin{array}{c}\text { Akzentuierung der Unterschiede } \\
\text { durch Wechselwirkungen mit }\end{array}$ & $\begin{array}{c}\text { Pfadabhängigkeit } \\
\text { Kontexterfahrungen über die Zeit (z.B. } \\
\text { Intelligenzunterschiede) }\end{array}$ \\
$\begin{array}{c}\text { wiei wissenschaftlichen Karrieren } \\
\text { (Matthäus-Effekt) }\end{array}$ \\
(vgl. DiPrete/Eirich 2006; Merton 2010)
\end{tabular}

Quelle: Diewald/Faist (2011: 106); eigene Darstellung. 
Diewald/Faist (2011) ergänzen die Perspektive sich „wechselseitig stützender und verstärkender Mechanismen" um Wechselwirkungen, die auf Konkurrenz, Konflikt und Substitution beruhen (106): Im Falle von Konkurrenz beeinträchtigen die hohen Anforderungen in einem Handlungsfeld das Engagement in einem anderen Handlungsfeld (ebd.). Die Steigerung der Konkurrenz stellt der Konflikt dar, bei dem das Engagement in einem Handlungsfeld, das Engagement in einem anderen ausschließt. Von Substitution sprechen Diewald/Faist (2011) hingegen dann, „wenn fehlende Handlungsmöglichkeiten in einem Feld durch verstärkte Engagements in anderen Feldern kompensiert werden, entweder um die gleichen Ziele auf anderem Wege oder um andere Ziele als die ursprünglich intendierten zu verfolgen“ (ebd). Vor diesem theoretischen Hintergrund werden der Freiwilligenarbeit als Handlungsfeld bislang vorwiegend kompensatorische bzw. substituierende Effekte, wie die der Integration und Inklusion sozial benachteiligter Bevölkerungsgruppen, zugeschrieben.

\section{(I) 3. Freiwilligenarbeit und soziale Ungleichheit}

Die Auseinandersetzung mit den bisher beschriebenen ungleichheitstheoretischen Ansätzen (vgl. Diewald/Faist 2011; Kreckel 2004; Solga et al. 2009) wirft unterschiedliche theoretische und empirische Fragen des Zusammenhangs von Freiwilligenarbeit und sozialer Ungleichheit auf. In einem ersten Schritt folgen Überlegungen zu der Frage, in welcher Form die Freiwilligenarbeit innerhalb der beschriebenen Strukturebenen sozialer Ungleichheit verortet werden und welche Schlüsse dadurch über das mögliche Zusammenwirken von Freiwilligenarbeit und sozialer Ungleichheit getroffen werden können. Im zweiten Schritt wird an den Ansatz von Diewald/Faist (2011) angeknüpft und der Blick auf die Freiwilligenarbeit als Kontext der Entstehung und Reproduktion sozialer Ungleichheit gerichtet.

\section{(I) 3.1. Freiwilligenarbeit als Determinante, Dimension und Auswirkung sozialer Ungleichheit}

Freiwilligenarbeit kann wie in nachfolgender Abbildung 6 dargestellt auf unterschiedliche Weise und abhängig von der analytischen Perspektive und prozessualen Interpunktion mit sozialer Ungleichheit im Zusammenhang stehen. 
Abbildung 6: Theoretische Verortung von Freiwilligenarbeit im Kontext sozialer Ungleichheit
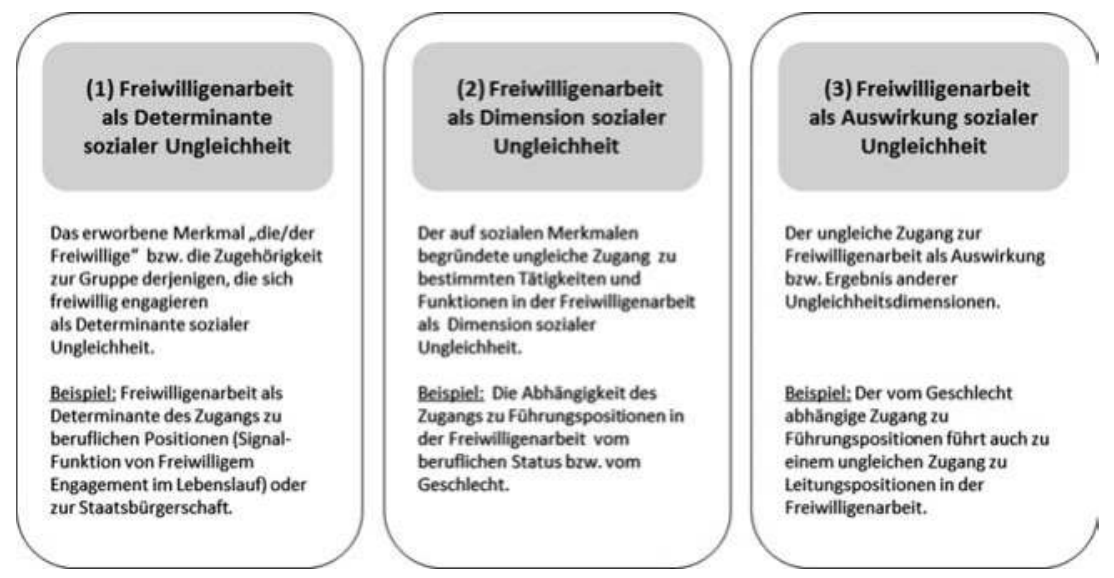

1. Freiwilliges Engagement kann als ein erworbenes soziales Merkmal die Zugehörigkeit zur sozialen Gruppe der Freiwilligen kennzeichnen und damit eine Determinante sozialer Ungleichheit darstellen.

2. Freiwilligenarbeit als Tätigkeit innerhalb formaler Organisationen, die mit sozialen Positionen verbunden sind und deren Zugang aufgrund sozialer Merkmalszugehörigkeit eingeschränkt ist, kann eine Dimension sozialer Ungleichheit darstellen.

3. Aus einer dritten Perspektive kann der Zugang zur Freiwilligenarbeit auch als Auswirkung bzw. Ergebnis bestimmter Ungleichheitsdimensionen angesehen werden. Nachfolgend wird auf die einzelnen Perspektive im Detail eingegangen und im Anschluss die Anforderungen an künftige empirische Erhebungen und Forschungsansätze diskutiert.

\section{(I) 3.1.1. Freiwilligenarbeit als Determinante sozialer Ungleichheit}

Die Bezeichnung „Freiwillige“ bzw. „Freiwilliger“ als erworbenes soziales Merkmal ist immer dann von Bedeutung, wenn damit die Zugehörigkeit zur sozialen Gruppe der Freiwilligen gemeint ist, und dies wiederum zu einem eingeschränkten oder bevorzugten Zugang zu sozialen Positionen und/oder erstrebenswerten sozialen und symbolischen Gütern (z.B. Zugang zu bestimmten Erwerbsfeldern, Führungspositionen, der Staatsbürgerschaft, Handlungsmöglichkeiten im Alter) führt (vgl. Solga et al. 2009: 16). In diesem Kontext ist z.B. aktuell die Frage interessant, in welchem Ausmaß der Nachweis von Freiwilligenarbeit im Lebenslauf und in Motivationsschreiben von den Bewerbern 
zum sogenannten résumé building eingesetzt wird (vgl. Handy et al. 2010). Auf Seite der Organisationen stellt sich die Frage, ob und in welchem Ausmaß Freiwilliges Engagement tatsächlich bei der Personalauswahl als Indikator für z.B. soziale Kompetenz und eine überdurchschnittliche Arbeitsbereitschaft an Bedeutung gewinnt.

\section{Abbildung 7: Freiwilligenarbeit als Determinante sozialer Ungleichheit}

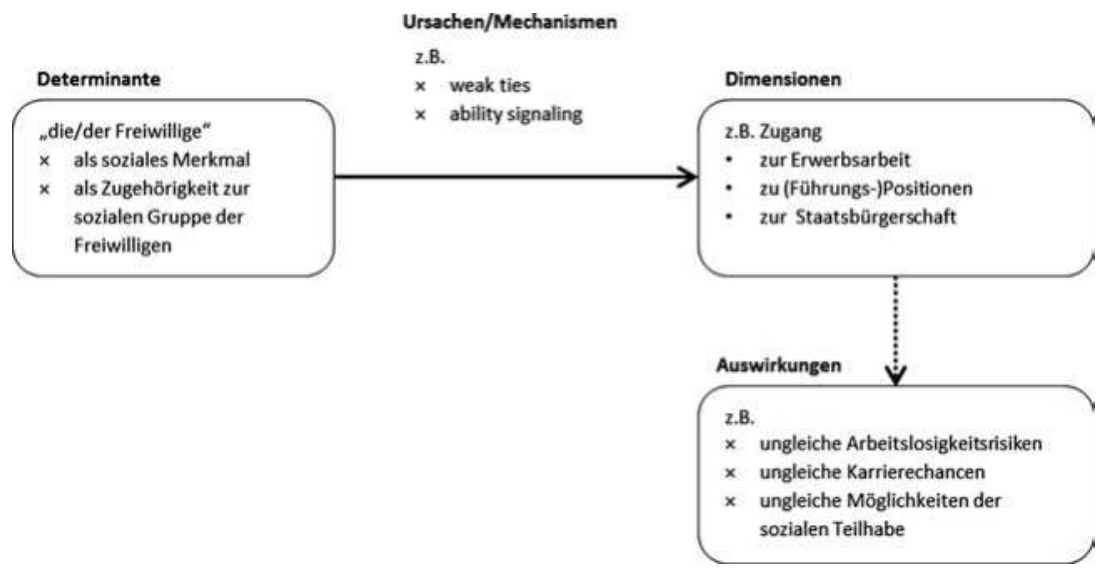

Freiwilligenarbeit fungiert hier als sogenanntes ability signaling, d.h. sie wird bewusst oder unbewusst als Nachweis für z.B. soziale Kompetenz oder für besonderes Engagement eingesetzt bzw. von den Personalverantwortlichen als solche interpretiert. Es scheint daher zunehmend einen Unterschied zu machen, ob man als Freizeitaktivität Schach spielt, Sport treibt oder sich freiwillig im Sozialbereich engagiert. In welcher Form Freiwilliges Engagement den Zugang zu bestimmten sozial erstrebenswerten Gütern und Positionen tatsächlich einschränkt oder fördert, bedarf weiterer empirischer Untersuchungen. Mit Freiwilligenarbeit, verstanden als Zugehörigkeit zu sozialen Netzwerken, ist auch die Nutzung des damit verbundenen sozialen (weak ties) wie symbolischen Kapitals (ability signaling) verbunden

\section{(I) 3.1.2. Freiwilligenarbeit als Dimension sozialer Ungleichheit}

In dem man die Perspektive auf Freiwilligenarbeit als Tätigkeiten innerhalb formaler Organisationen verlegt, geraten die damit einhergehenden sozialen Positionen in den Fokus. Ist der Zugang für bestimmte Personen aufgrund ihrer Zugehörigkeit zu sozialen Merkmalsgruppen (z.B. Geschlecht, Alter, Bildungsschicht) eingeschränkt, stellt dies in erster Instanz einmal „nur“ einen ungleichen 
Zugang zur Freiwilligenarbeit dar. Ausgehend von der Abgrenzung zwischen sozialer Ungleichheit und sozialer Differenzierung (vgl. Kreckel 2004; Schwinn 2007; Solga et al. 2009) ist damit die zentrale Frage, ob mit dem sozial ungleichen Zugang zur Freiwilligenarbeit im Allgemeinen, wie zu den verschiedenen Feldern und (hierarchischen) Positionen im Besonderen, auch Vor- und Nachteile, d.h. Asymmetrien in den Handlungsmöglichen der Individuen verbunden sind. D.h. ob die formelle Freiwilligenarbeit als eine Form der organisationalen Ein- bzw. Anbindung sozialstrukturierende Wirkung und damit soziale Ungleichheit generierende Bedeutung hat. Denn wie bereits beschrieben, müssen theoretisch wie empirisch nicht zwangsläufig alle Heterogenitäten zwischen Menschen zu sozialer Ungleichheit führen (vgl. Diewald/Faist 2011: 96; Solga et al. 2009: 16).

Abbildung 8: Freiwilligenarbeit als Dimension sozialer Ungleichheit

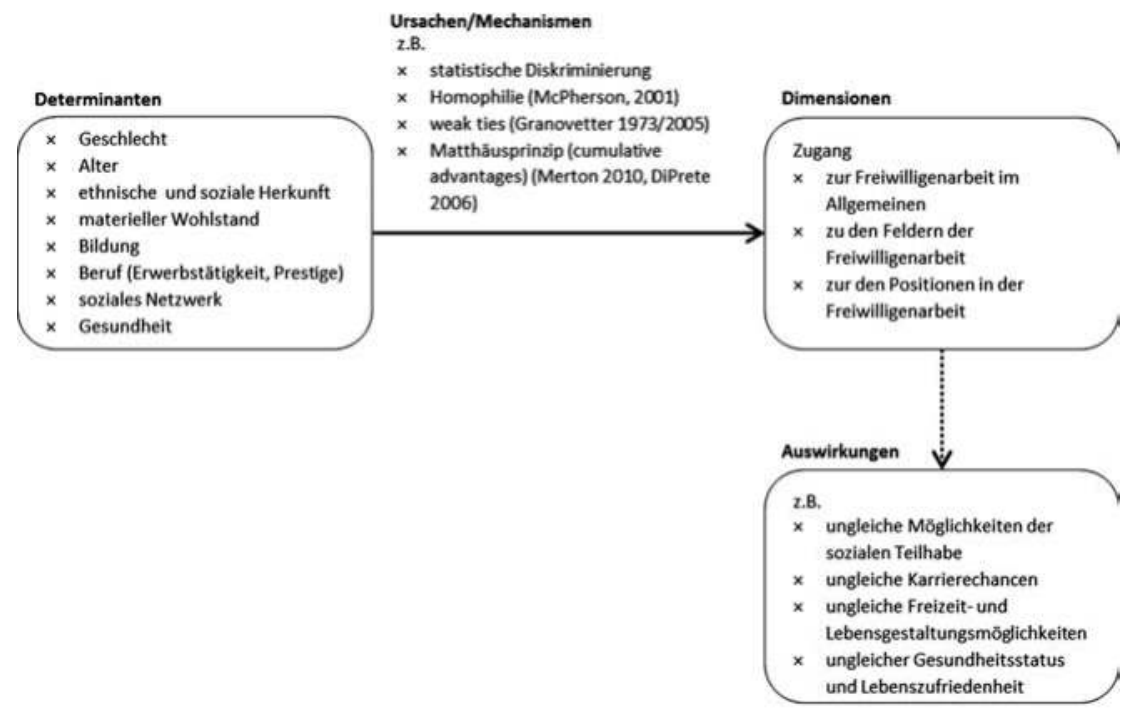

Theoretisch wie empirisch müssen daher sowohl die Determinanten, die Mechanismen als auch die Auswirkungen des ungleichen Zugangs zur Freiwilligenarbeit spezifiziert und empirisch überprüft werden. Erst dann handelt es sich tatsächlich um eine Dimension sozialer Ungleichheit. Somit ist die alleinige empirische Beobachtung, dass bestimmte Personengruppen eher Freiwilligenarbeit leisten als andere, ohne den Blick auf die Auswirkungen des ungleichen Zugangs für die Feststellung sozialer Ungleichheit unzureichend und würde vorerst nur auf Heterogenitäten verweisen. Zum Beispiel wäre die Beobachtung, dass manche Personen 
eine ehrenamtliche Vorstandsposition im Sport- oder Kulturverein innehaben und andere wiederum freiwillig und unbezahlt einen Besuchsdienst im Pflegeheim leisten, ohne den Blick auf den sozial strukturierten Zugang, die herkunftsabhängigen habituellen Präferenzen sowie auf die nichtmonetären symbolischen, sozialen und kulturellen Profite der jeweiligen Tätigkeiten alleine noch kein Indiz dafür sein, dass es sich hier um eine Form sozialer Ungleichheit handelt.

Erst mögliche überindividuelle empirische Ergebnisse, z.B. dass Frauen unabhängig vom Alter, Bildungsgrad und Berufsstatus in bestimmten Feldern der Freiwilligenarbeit unterrepräsentiert sind und dabei nochmals seltener in Positionen mit leitenden Funktionen zu finden sind, würden darauf schließen lassen, dass wie in der Erwerbsarbeit auch in der Freiwilligenarbeit gläserne Wände und Decken existieren. Als mögliche Ursachen können ungleichheitsgenerierende Mechanismen wie jener der statistischen Diskriminierung, das Prinzip der Homophilie (McPherson et al. 2001), der weak ties (Granovetter 1973, 2005) sowie der schon mehrfach erwähnten Matthäus-Effekt (DiPrete/ Eirich 2006; Merton 2010) wirksam sein. Die zentrale Frage ist somit, welche Personengruppen haben aufgrund welcher Merkmale bzw. Kapitalausstattungen Zugang zu spezifischen Positionen (z.B. leitende Funktion, administrative Funktion, ausführende Funktion, unterstützende Funktion etc.) in der Freiwilligenarbeit bzw. sind davon ausgeschlossen und welche Vor- und Nachteile sind damit in anderen Lebensbereichen verbunden. Wie im Kapitel (II) zum Stand der Forschung nachfolgend noch gezeigt wird, gibt es im Gegensatz zum sozialen ungleichen Zugang zur Freiwilligenarbeit im Allgemeinen nur wenige empirische Befunde zum Zugang zu freiwilligen bzw. ehrenamtlichen Leitungs- bzw. Führungspositionen. Es liegt jedoch nahe, dass Ehrhardt mit seinen Überlegungen ausgehend von Weber (1920) in vielen Bereichen Recht behalten dürfte:

„Je höher die soziale Wertschätzung und Bedeutung des Amtes, desto höher der dafür notwendige soziale Status, um das Amt besetzen zu können (Zugangsvoraussetzungen). Dass dieser Zusammenhang für Elite- bzw. für herausragende Ämter, wie etwa den Vorstandsvorsitz der Deutschen Welthungerhilfe oder des Deutschen Kinderhilfswerkes, gilt, liegt auf der Hand [...] Aber man wird auch davon ausgehen können, dass dieser Zusammenhang ebenso für mittlere Positionen - etwa für den $>$ Zweiten Vorsitz $<$ eines Tierheimes oder eines kleinen Sportvereins - gilt. Der soziale Status und das Amt passen - insgesamt gesehen - zusammen“" (Ehrhardt 2011: 62f).

Ehrhardt (2011) vertritt diesbezüglich jedoch auch die These, dass gerade auch aufgrund bestehender Abweichungen zwischen beruflicher Position und ehrenamtlicher Position Freiwilligenarbeit eine eigene Dimension sozialer Ungleichheit darstellen kann. 
„Geht man von diesem Zusammenhang aus, dann ist das gehobene und mittlere Ehrenamt eine Möglichkeit, sozialen Status öffentlich darzustellen, ebenso wie etwa ein distinguierter Kunstgeschmack oder bestimmte Accesoires (z.B. Auto, Haus). Theoretisch interessanter und wohl auch realitätsnäher ist es, geringe Abweichungen zuzulassen; nimmt man also an, dass das Berufsprestige und das Prestige, das sich aus einer ehrenamtlichen Tätigkeit ergibt, nicht in einem >perfekten $<$ Zusammenhang stehen, sondern in einem gewissen, eingeschränkten Maße Personen auch innerorganisatorisch aufsteigen können, dann müsste das Ehrenamt - zumindest bei den mittleren und gehobenen Positionen - als ein eigenständiger Faktor bei der Verortung im Schichtungsgefüge betrachtet und neben den klassischen Merkmalen (Beruf, Bildung, Einkommen, Vermögen [...]) in den soziologischen Analysen [berücksichtigt werden]“ (Ehrhardt 2011: 63f).

Im Gegensatz zur Erwerbsarbeit, wo dem ungleichen Zugang vielfach eindeutige Nachteile wie geringeres Einkommen, schlechtere Arbeitsbedingungen und vergleichsweise inferiore Karrieremöglichkeiten zuzuordnen sind, müssen bei der Freiwilligenarbeit die Nachteile des ungleichen Zugangs erst kausal nachgewiesen werden. Empirische Befunde, dass mit der Freiwilligenarbeit und den dabei geleitesten Tätigkeiten, den damit verbundenen sozialen Netzwerken, den dabei gemachten Erfahrung und erworbenen Kompetenzen sowie dem bei leitenden Positionen und Vorstandsfunktionen erworbenen symbolischen Status ursächlich Vorteile verbunden sind, fehlen vielfach mangels entsprechender Längsschnittdaten. Die empirische Überprüfung der „Ungleichheitsdimensions-These der Freiwilligenarbeit“ bedarf demnach eines umfangreichen, kausalanalytischen empirischen Forschungsprogramms, das über die aktuelle Form der Surveys zur Freiwilligenarbeit hinausreicht. Wie im Kapitel (II) zum Stand der Forschung noch gezeigt wird, ist der Forschungsstand zu den Auswirkungen, d.h. der Vor- und Nachteile des Zugangs zur Freiwilligenarbeit bislang eher theoretisch-interpretativer denn kausalanalytischer Art. Der eingeschränkte Zugang zur Freiwilligenarbeit und die vom Status abhängige hierarchische Positionszuweisungen in den Freiwilligenorganisationen dürfte jedenfalls ungleichheitsgenerierende als auch -reproduzierend Wirkungen entfalten.

\section{(I) 3.1.3. Freiwilligenarbeit als Auswirkung sozialer Ungleichheit}

Bei dieser Perspektive wird bewusst auf eine grafische Darstellung verzichtet, da es lediglich von der kausallogischen sowie theoretischen Interpunktion abhängt, ob es sich bei einem Phänomen um eine Dimension oder eine Auswirkung sozialer Ungleichheit handelt (Solga et al. 2009). Ausgehend von Abbildung 8 ist es daher auch möglich, je nach Fokus des Forschungsinteresses Freiwilligenarbeit als Auswirkungen z.B. (1) eines ungleichen Zugangs zu materieller Absicherung 
und damit zu, „von ökonomischen Zwängen befreite[r] Zeit“ (Bourdieu 1983, S. 188), (2) eines ungleichen Zugangs zum Bildungssystem und damit verbunden ungleichen Kompetenzen und Ressourcen (vgl. Wilson 2012) sowie (3) einer ungleichen Ausstattung an sozialem wie auch (feldspezifisch wirksamen) symbolischem Kapital zu betrachten. Der Unterschied zur vorangegangen Perspektive - den ungleichen Zugang zur Freiwilligenarbeit selbst als Dimension sozialer Ungleichheit zu untersuchen - ist, dass hier nicht der Prozess und die Mechanismen der Ungleichheitsgenese, die zum ungleichen Zugang führen, im Zentrum stehen, sondern Freiwilligenarbeit eine Auswirkung von anderen Ungleichheitsdimensionen darstellt.

\section{(I) 3.2. Freiwilligenarbeit als Kontextebene der Genese und Reproduktion sozialer Ungleichheit}

In der vierten Perspektive auf den Zusammenhang von Freiwilligenarbeit und sozialer Ungleichheit stellen die Freiwilligenorganisationen als formale Organisationen eine Kontexteben bzw. ein Feld der Genese und Reproduktion sozialer Ungleichheit (vgl. Diewald/Faist 2011) dar. In jenen Fällen, wo Organisationen des Dritten Sektors sowohl hauptamtlich Erwerbstätige als auch freiwillige Mitarbeiter beschäftigen, sind diese zwar ohnehin auch zu den Organisationen der Erwerbsarbeit zu zählen, jedoch sind im Rahmen der Ungleichheitsforschung bislang vordergründig die Positionen von Erwerbstätigen hinsichtlich Einkommen, Status und Prestige untersucht worden, nicht aber die Position von Freiwilligen bzw. Ehrenamtlichen. Wenn über die Bedeutung der differenzierten Institutionen für die Reproduktion sozialer Ungleichheit gesprochen wird, werden Freiwilligenorganisationen wie z.B. Vereine etc. zwar nicht explizit ausgenommen, jedoch bleiben sie bei der Benennung der bedeutsamen Institutionen und Felder bzw. Systeme fast immer unerwähnt (vgl. Diewald/Faist 2011; Rehberg 2011; Therborn 2006). Eine der wenigen Arbeiten die Freiwillige Vereinigungen als Orte der Netzwerkbildung und damit auch als Orte der Entstehung und Erhaltung sozialer Ungleichheit untersuchen, findet sich im Rahmen der Netzwerkforschung (vgl. Fuhse 2008). So sind Freiwillige Vereinigungen bzw. Vereine neben dem Arbeitsplatz, Bildungseinrichtungen, dem Wohnumfeld und informalen Treffpunkten zentrale Orte persönlicher Beziehungen und damit auch potentiell Orte sozialer Schließung ${ }^{21}$. In den Konzepten sozialer Ungleichheit wird Freiwilliges Engagement als Handlungsfeld bislang jedoch nicht explizit diskutiert. Diewald/Faist (2011) führen in ihrem Modell zwar Kinder- und

21 vgl. dazu auch die Forschung zu Homophilie (McPherson et al. 2001). 
Hausarbeit als bedeutsame Kontextebene an, die Freiwilligenarbeit bleibt in diesem Zusammenhang jedoch unerwähnt (vgl. Diewald/Faist 2011: 95). So gelten die Familie, das Bildungssystem, die Erwerbsarbeit bzw. das Beschäftigungssystem sowie der (Wohlfahrts-) Staat mit seinen Institutionen weiterhin als primäre Orte der Reproduktion sozialer Ungleichheit (vgl. Diewald/Faist 2011: 105f; Therborn 2006: 8). Schwinn (2008) fasst es unter Bezug auf Kreckel (2004) noch etwas enger:

„Den Kern der modernen Ungleichheitshierarchie bildet die ,meritokratische Triade von Bildung, Beruf und Einkommen (Kreckel 2004, S. 97). Für das Erringen der damit gesetzten Leistungskriterien sind drei institutionelle Felder zentral: Familie, Bildungsund Arbeitsmarktinstitutionen" (Schwinn 2008: 27).

Im Rahmen eines Sonderforschungsbereiches ${ }^{22}$ der $\mathrm{DFG}^{23}$ an der Universität Bielefeld mit dem Titel „Von Heterogenitäten zu Ungleichheiten“ wird die Bedeutung von Organisationen bei der (Re-)Produktion sozialer Ungleichheit untersucht („Von Heterogenitäten zu Ungleichheiten im Kontext von Organisationen").

„(1) Weil Organisationen über formale Positionsstrukturen und Regelsysteme Interaktionen kanalisieren, tragen sie zu Grenzziehungen bei und nehmen Differenzierungen über die $\mathrm{Zu}-$ und Verteilung von Belohnungen und Belastungen vor. Damit transformieren sie nicht nur individuelle Heterogenitäten in bewertete Unterschiede, sie eröffnen auch entlang organisationsintern bewerteter Unterscheidungsmerkmale selektive Verwirklichungschancen. [...]

(2) In dem Maße wie Organisationen selbst einer horizontalen Differenzierung ausgesetzt sind und ihren Mitgliedern unterschiedliche Vorteile gewähren, wird es für die Zugangs- und Verwirklichungschancen einer Person wichtiger, in welcher Organisation sie zu welchem Zeitpunkt ihres Lebensverlaufs eingebunden ist und in welcher Entwicklungsphase sich diese Organisation befindet. [...]

(3) Sowohl Bildungs- als auch Arbeitsorganisationen sind institutionalisierte Handlungskontexte, in denen Zuschreibungen vermittelt und perpetuiert werden ${ }^{\text {(224. }}$.

Es ist anzunehmen, dass mit dem Begriff Arbeitsorganisationen Freiwilligenorganisationen per se nicht ausgeschlossen sind, in welchem Umfang diese jedoch in ihren Besonderheiten in die theoretische wie empirische Forschung miteingeschlossen werden, bleibt abzuwarten. Mit der zunehmenden Bedeutung des Konzepts der Zivilgesellschaft, der darin agierenden Organisationen sowie der

22 SFB 882 (Sonderforschungsbereich 882).

23 Deutsche Forschungsgemeinschaft.

24 SFB 882, Projektbereich B; Quelle: http://www.sfb882.uni-bielefeld.de/de/areas abgerufen am 04.03.2014. 
bezahlten wie unbezahlten Akteure sowohl in der Forschung (vgl. Anheier 2013; Salamon et al. 2000) als auch in den politischen Agenden (z.B. Europäische Jahr der Freiwilligentätigkeit zur Förderung der aktiven Bürgerschaft 2011) sollten jedoch auch die darin wirksamen Prozesse und Mechanismen sowie deren Wechselwirkungen mit anderen Bereichen der Gesellschaft in den Fokus des Interesses rücken. Die vordergründige Ausklammerung der Freiwilligenarbeit aus ungleichheitstheoretischen Überlegungen ist aufgrund der gesellschaftlichen Konnotierung mit Attributen wie freiwillig und uneigennützig auf den ersten Blick nachvollziehbar. Auch stellt Freiwilligenarbeit im theoretischen wie praktischen Sinn selbst kein eigenes gesellschaftliches Feld bzw. System dar, sondern ist Teil jeweils höchst unterschiedlicher gesellschaftlicher Felder wie z.B. des Sozial- und Gesundheitsbereichs, des Sports, der Religion, der Politik, der Bildung, etc. Die Frage ist, in welchen dieser gesellschaftlichen Felder die Freiwilligenarbeit durch soziale Schließungsprozesse und interne Hierarchisierung soziale Ungleichheit befördert und welche Ursachen dafür verantwortlich gemacht werden können. Ein theoretischer Ansatz, der sich in seinem Kern mit den Regeln und Prozessen des (ungleichen) Zugangs zu unterschiedlichen gesellschaftlichen Feldern sowie deren jeweiligen internen (hierarchischen) Strukturierung auseinandersetzt, ist die bereits erwähnte Sozialtheorie Pierre Bourdieus (1977, 1982). Darüber hinaus ermöglicht die Theorie Bourdieus, durch die Erweiterung des bislang vorwiegend ökonomisch verstandenen Kapitalbegriffs (Marx/Engels 1977 (1848)) um eine soziale, eine kulturelle und eine symbolischen Dimension, die Analyse von gesellschaftlichen Bereichen, die auf den ersten Blick der ökonomischen Sphäre entrückt erscheinen. Prozesse sozialer Schließung, Hierarchisierung und Ausbeutung finden damit nicht nur im Feld der Erwerbsarbeit, sondern auch in den sozialen und kulturellen Sphären statt. Im nächsten Kapital wird dazu die Theorie Pierre Bourdieus kurz eingeführt und ausgewählte Perspektiven auf die Freiwilligenarbeit und dabei vermutete Prozesse und Ursachen sozialer Selektion und interner Hierarchisierung diskutiert.

\section{(I) 4. Theoretische Perspektiven zur sozialen Schließung und Hierarisierung in der Freiwilligenarbeit}

Pierre Bourdieu $(1977,1982)$ war einer der wenigen Theoretiker, der soziale Ungleichheit bei all seinen Themen zentral mitgedacht hat (Weiß 2004: 210). Somit leisten seine theoretischen Grundlagen und Überlegungen einen fruchtbaren Beitrag zur Beantwortung der Frage nach der Rolle von formeller Freiwilligenarbeit im Zusammenhang mit der Genese und Reproduktion sozialer Ungleichheit. Bourdieu (Bourdieu 1977, 1982, 1983) versteht individuelle Akteure als 
Produzenten sozialer Praxis. Durch ihr Handeln, geprägt durch den jeweiligen Habitus und abhängig von der Ressourcenausstattung (z.B. an ökonomischen, kulturellen, sozialen Kapitalien), (re-)produzieren die Individuen durch die sozialen Kämpfe um begehrte Positionen, die Praxisformen in den unterschiedlichen Lebensbereichen (Felder). Die Kapitalien werden in unterschiedlichem Ausmaß in Abhängigkeit der sozialen Herkunft teilweise vererbt oder aber im Laufe des Lebens in den verschiedenen Feldern akkumuliert. Der Wert der Kapitalien variiert je nach Feld (z.B. Wirtschaft, Religion, Sport, Familie, etc.). Die Positionen, die die Akteure in den unterschiedlichen sozialen Feldern einnehmen, sind einerseits von der Kapitalausstattung, andererseits vom Wert abhängig, d.h. vom symbolischen Kapital, das den Kapitalien im jeweiligen Feld zuerkannt wird. Demzufolge kann ein und dieselbe Person in den verschiedenen sozialen Feldern mitunter auch verschiedene Positionen einnehmen. Mit den Praxisformen selbst sowie dem „Kampf“ um die jeweils in einem Feld erstrebenswerten Positionen werden vorwiegend implizit auch die Regeln (Bedeutung der Kapitalien) in den Feldern (re-)produziert.

Bourdieus Bestreben war es, Bereiche bzw. Felder, die soziale Ungleichheit primär im Außen jedoch nicht im Innen verortet, kritisch in Hinblick auf deren impliziten Beitrag zur Reproduktion sozialer Ungleichheit zu beleuchten.

„Bourdieu kritisiert das Postulat der Uneigennützigkeit, die von der Wirtschaftstheorie den verschiedenen kulturellen, sozialen und symbolischen Praxisformen unterstellt wird, und hebt demgegenüber die polymorphen Interessen, Einsätze und Profitmöglichkeiten in den unterschiedlichsten gesellschaftlichen Feldern hervor" (Schwingel 2000: 85 nach Fuchs-Heinritz/König 2005: 158).

Weiß (2004) betont, dass

„Bourdieu wesentliche Teile seines Lebenswerks darauf verwandt [hat], eine Klassenstruktur des sozialen Raumes selbst dort nachzuweisen, wo nicht einmal der aufgeklärteste Beobachter soziale Ungleichheit vermutet hätte“ (Weiß 2004: 210).

Bourdieu hat sich in seinen Arbeiten zentral mit dem auf den ersten Blick ebenfalls der ökonomischen Sphäre fernen Bildungssystem und dessen Funktion bei der Aufrechterhalten des sozial ungleichen, klassenabhängigen Zugangs zu hierarchischen Positionen beschäftigt (Bourdieu 2000 (1973)). Im Fokus stehen die Ursachen und Prozesse des bis heute fortbestehenden Unvermögens des Bildungssystems, den Zugang zu (höheren) Bildungstitel von der sozialen Herkunft zu entkoppeln. Es ist vor allem „Bourdieus Verdienst, gezeigt zu haben, wie kulturelle Praktiken untergründig immer mit einer Herrschaftsdimension verbunden sind“ (Rehberg 2011: 14). Weiß (2004) formuliert dazu ergänzend, bei 
„Bourdieu liegt Ungleichheit der sozialen Welt insgesamt zugrunde. Die Gesellschaft entfaltet sich nicht zufällig, sondern im Rahmen ihrer historisch (und damit auch herrschaftsförmig) hervorgebrachten Möglichkeiten. Die Chancen, diese Möglichkeiten zu nutzen, sind ungleich auf Individuen und Gruppen verteilt und werden von Bourdieu als Mehrzahl von Kapitalsorten benannt“(Weiß 2004: 211).

Für Bourdieu zeigt sich soziale Ungleichheit in den verschiedenen Lebensbedingungen (d.h. dem Volumen und der Struktur der Kapitalien), Habitusformen und schlussendlich in den Lebensstilen (vgl. Fuchs-Heinritz/König 2005: 187). Freiwilligenarbeit kann daher einerseits als Ausdruck bzw. Bestandteil eines (distinguierten) Lebensstils betrachtet werden und andererseits als Handlung bzw. Tätigkeit bei der primär soziale, symbolische und kulturelle Kapitalien akkumuliert werden.

Bevor der Versuch unternommen wird Bourdieus Theoreme und Überlegung fruchtbringend auf die Frage nach der Reproduktion sozialer Ungleichheit durch die Freiwilligenarbeit anzuwenden, werden die grundlegenden Aspekte und Begrifflichkeiten seiner Sozialtheorie kurz vorgestellt.

\section{(I) 4.1. Grundlagen der Sozialtheorie Pierre Bourdieus}

\section{(I) 4.1.1. Formen des Kapitals}

Bourdieu $(1982,1983)$ wendet den Kapitalbegriff, der bisher (z.B. bei Marx) nur im ökonomischen Sinne verwendet wurde, auf den kulturellen und den sozialen Bereich an. Er arbeitet in seinen Theorien mit den Begriffen des ökonomischen, kulturellen und sozialen Kapitals, teilweise erweitert um das symbolische Kapital. Im Laufe eines Lebens kommt es zu einer Akkumulation aller drei Kapitalsorten. Diese Anhäufung von Kapital ist stark von der sozialen Herkunft und den Sozialisationsbedingungen bestimmt. Dieses Konzept steht somit im Gegensatz $\mathrm{zu}$,dem Bild eines Universums vollkommener Konkurrenz und Chancengleichheit, einer Welt ohne Trägheit, ohne Akkumulation und ohne Vererbung von erworbenen Besitztümern und Eigenschaften“" (Bourdieu 1983: 183). Obwohl es auf der einen Seite der Investition von Zeit bedarf, um Kapital zu erwerben, hat es auf der anderen Seite auch eine eigene Überlebenstendenz, d.h. es kann sich "selbst reproduzieren oder auch wachsen“ (Bourdieu 1983: 183) ${ }^{25}$. Bestimmte Kapitalsorten wie zum Beispiel Geld oder Eigentum können von einer Generation zur nächsten einfacher und direkter, andere wie Bildung schwerer und meist

25 vgl. dazu auch Forschungsarbeiten zum sogenannten Matthäus-Effekt (Merton 2010) bzw. zu kumulativen Vorteilen (cumulative advantages) (DiPrete/Eirich 2006). 
nur indirekt weitergegeben werden. Neben der Kapitalakkumulierbarkeit ist die Kapitaltransformierbarkeit gerade auch für die Erklärung der Entstehungs- und Reproduktionsmechanismen sozialer Ungleichheit von zentraler Bedeutung (vgl. dazu Kreckel 2004: 75ff; Schwinn 2008: 36; Weber 1921/1980: 531ff). Es ist daher möglich, auch wenn es mit Kosten bzw. Arbeit verbunden ist, ein bestimmtes Kapital in ein anderes umzuwandeln. Man kann zum Beispiel Geld in Bildung konvertieren, jedoch bedarf es dazu einer gewissen Transformationsarbeit (wie dem Besuch einer Schule oder einer Universität), die geleistet werden muss. Grundsätzlich ist „, [j]ede Art von Kapital [...] an ein Feld gebunden und hat die gleichen Gültigkeits- und Wirksamkeitsgrenzen wie das Feld, in dem es Geltung hat" (Bourdieu 2001a: 52; vgl auch Weiß 2004: 212). Dieses Gesetz ist jedoch insofern auszuweiten, als dass eine bestimmte Kapitalform durchaus für den Zugang zu anderen Felder von Bedeutung sein kann. In diesem anderen Feld kommt es jedoch nicht zu dessen weiterer Akkumulation. Zum Beispiel ist im Feld der Wirtschaft zwar vielfach kulturelles Kapital in Form von Bildungstiteln für den Zugang zu Führungspositionen relevant, akkumuliert wird dann allerdings primär ökonomisches und symbolisches Kapital, nicht jedoch kulturelles Kapital.

\section{Kulturelles Kapital}

Bourdieu untergliedert das kulturelle Kapital in inkorporiertes, objektiviertes und institutionalisiertes Kapital. Wie die Bezeichnung inkorporiert bereits andeutet, handelt es sich hierbei um „körpergebundenes“ Kapital, das einen „Verinnerlichungsprozeß“ voraussetzt, d.h. die Akkumulation „kostet Zeit“ in Form von „Unterrichts- und Lernzeit“ (Bourdieu 1983: 186). Es ist wichtig, dass diese Art des Kapitals nur von seinem Träger selbst erworben werden kann, somit „das Delegationsprinzip“ (ebd.) ausschließt, und „zu einem festen Bestandteil der ,Person', zum Habitus“ (ebd.: 187) wird. Inkorporiertes Kulturkapital kann nicht kurzfristig weitergegeben, z.B. vererbt oder verschenkt werden, ist aber trotzdem in der Qualität und Quantität von der sozialen Herkunft, konkret von der familiären Kapitalstruktur, abhängig. Zum Beispiel ist „der schulische Ertrag schulischen Handelns vom kulturellen Kapital abhängig, das die Familie zuvor investiert hat" (Bourdieu 1983: 186). In welchem Umfang der Schulbesuch zur Anhäufung von inkorporiertem Kapital einer Person beiträgt, wird vom bisher erworbenen Kapital bestimmt. Die Weitergabe von kulturellem Kapital innerhalb der Familie ist meist nicht so offensichtlich wie die Weitergabe von ökonomischem Kapital in Form von Geld oder Besitz und daher „die am besten verschleierte Form der erblichen Übertragung von Kapital“ (Bourdieu 1983: 
188). Der Zeitpunkt in der Kindheit, an dem der Akkumulationsprozess beginnt, ist vom kulturellen Kapital der Herkunftsfamilie abhängig und ist der Ursprung für spätere Unterschiede im Umfang des kulturellen Kapitals. Die Dauer dieses Prozesses hängt aber auch davon ab, wie lange die „Familie freie, von ökonomischen Zwängen befreite Zeit garantieren kann“ (ebd.).

Objektiviertes Kulturkapital kann in Form seiner materiellen Träger (Gemälde, Denkmäler, Instrumente, Bücher) direkt weitergegeben werden (vgl. Bourdieu 1983: 188). Nach Bourdieu „ist allerdings nur das juristische Eigentum“ übertragbar, für die „eigentliche Aneignung“ (ebd.) ist verinnerlichtes Kapital notwendig. Um zum Beispiel den Wert eines Instrumentes nutzen zu können, muss man auch die Fähigkeit haben, es zu bespielen. Auch für den Genuss eines Gemäldes ist ein bestimmtes inkorporiertes kulturelles Kapital notwendig.

Um innerhalb der Gesellschaft den Besitz von inkorporiertem Kulturkapital vergleichbar zu machen und ihm einen ökonomischen Wert zuordnen zu können, wird es in Form von (Bildungs-)Titeln institutionalisiert. Diese sind sowohl „schulisch sanktioniert und rechtlich garantiert“, als auch „(formell) unabhängig von der Person ihres Trägers“ (Bourdieu 1983:190). Ein Bildungstitel besitzt einen gewissen Wert, der nicht notwendigerweise dem wirklich verinnerlichten Kapital entsprechen muss. Durch schulische oder akademische Titel wird Anerkennung für kulturelles Kapital verliehen und es wird auch möglich „die Besitzer derartiger Titel zu vergleichen und sogar auszutauschen“ (ebd.: 190). Der Erwerb eines Titels ist mit ökonomischem Aufwand verbunden, dafür aber nach einem bestimmten Wechselkurs auch wieder in ökonomisches Kapital konvertierbar, in dem jenem Titel am Arbeitsmarkt ein gewisser Geldwert gegenübersteht. Durch „die Bildungsexpansion und die Titelinflation“ kann es sein, „daß die Investitionen an Zeit und Anstrengungen sich als weniger rentabel herausstellen" (ebd.:190), d.h. die immerwährende Umwandelbarkeit zu einem bestimmten Wechselkurs nicht garantiert ist.

\section{Ökonomisches Kapital}

Im Gegensatz zum kulturellen Kapital ist „das ökonomische Kapital [...] unmittelbar und direkt“, also ohne großen Transformationsaufwand, „in Geld konvertierbar und eignet sich besonders gut zur Institutionalisierung in der Form von Eigentumsrecht" (Bourdieu 1983: 185). Zum ökonomischen Kapital zählen, neben Geld, alle Arten des materiellen Besitzes und des Eigentums. Es wird oft auch als dominantes Kapital angesehen, obwohl die anderen Kapitalformen weitgehend gleichgestellt sind (vgl. Fröhlich 1994: 36). 


\section{Soziales Kapital}

Neben den bereits beschrieben Kapitalsorten können auch Beziehungen zu anderen Personen oder die Zugehörigkeit zu einer bestimmten Gruppe als Kapital bezeichnet werden. Persönliche Kontakte und/oder die Mitgliedschaften in exklusiven Vereinigungen können indirekt in kulturelles und/ oder ökonomisches Kapital umgewandelt werden, indem die Vorteile von gewissen sozialen Strukturen genutzt werden. So bezeichnet Bourdieu das soziale Kapital ${ }^{26}$ als

„die Gesamtheit der aktuellen und potentiellen Ressourcen, die mit dem Besitz eines dauerhaften Netzes von mehr oder weniger institutionalisierten Beziehungen gegenseitigen Kennens oder Anerkennens verbunden ist; oder, [...] die auf der Zugehörigkeit zu einer Gruppe beruhen“ (Bourdieu 1983: 190f).

Diese Beziehungsnetze bedürfen permanenter „Beziehungsarbeit“ (Bourdieu 1983: 193) und sind Produkt „individueller und kollektiver Investitionsstrategien“ (ebd.: 192) mit dem Ziel, früher oder später daraus einen Nutzen zu ziehen. Augenscheinlich wird der Wert des sozialen Kapitals dort, wo Personen aus ähnlichem kulturellen und ökonomischen Kapital, auf Grund ihrer unterschiedlichen Beziehungsnetze ungleiche Erträge generieren (vgl. Bourdieu 1983: 191). In Gruppen kommt es oftmals zu einer Konzentration von sozialem Kapital, indem es an eine bestimmte Person delegiert wird. Bourdieu spricht dann von mehr oder weniger institutionalisierter Delegation, je nachdem, wie genau die Verantwortungsbereiche der einzelnen Gruppenmitglieder abgegrenzt sind (vgl. 1983, S. 193f). Die Macht die jenem Repräsentanten der Gruppe zugeschrieben wird, kann dieser dann aber folglich auch über die Gruppe ausüben, wenn nicht sogar gegen sie verwenden (vgl. Bourdieu 1983: 193ff).

\section{Symbolisches Kapital}

Symbolisches Kapital beruht auf Anerkennung und Bekanntheit. In ihm drückt sich aus, wie die Zusammensetzung und der Umfang der drei Kapitalsorten (kulturelles, ökonomisches, soziales) einer Person von den anderen Akteuren

26 Im Kontext der Engagement- und Freiwilligenforschung ist an dieser Stelle auf die unterschiedliche Konzeption bzw. Definition von Sozialkapital bzw. sozialem Kapital hinzuweisen (z.B. wie bei Robert Putnam (1993)) (vgl. Braun 2003a). Weiterführend zu den unterschiedlichen Sozialkapitalkonzepten siehe einschlägige Forschungsarbeiten (Adler/Kwon 2002; Braun 2001b, 2003a; Brunie 2009; Coffé/Geys 2007; Coleman 1988; Schneider 2009; Seubert 2009). 
im jeweiligen Feld wahrgenommen, bewertet und als legitim anerkannt wird (vgl. Fröhlich 1994: 37). „Das symbolische Kapital besteht aus den Chancen, soziale Anerkennung und soziales Prestige zu gewinnen und zu erhalten“" (FuchsHeinritz/König 2005: 169).

„Die Idee, daß die Kämpfe um Anerkennung eine fundamentale Dimension des sozialen Lebens bilden, daß es darin um Akkumulation einer besonderen Art von Kapital geht - eben $>>$ Ehre $<<$ im Sinne von Reputation, Prestige; daß es folglich eine spezifische Logik der Akkumulation von symbolischem, d.h. auf Bekanntheit und Anerkennung begründetem Kapital gibt; [...]“ (Bourdieu 1992b: 37).

Dabei steigt das Interesse am symbolischen Gewinn einer Tätigkeit, je weiter diese von Zwangsarbeit entfernt und je näher sie scholastischen Tätigkeiten kommt (vgl. Bourdieu 2001b: 260). Damit ist Freiwilligenarbeit zumindest theoretisch prädestiniert als Quelle symbolischen Kapitals und als Dimension sozialer Ungleichheit.

Ergänzend ist anzumerken, dass die vier zentralen Kapitalarten von Bourdieu in seinen Analysen teilweise um feldspezifische Kapitalien, wie z.B. politisches Kapital, sportives Kapital, religiöses Kapital, etc. ergänzt werden. Diese Kapitalarten bezeichnen ebenfalls „akkumulierte Arbeit bzw. Investitionen“, sind jedoch in ihrer Wirksamkeit und Bedeutung eng an die jeweiligen Felder gebunden.

\section{(I) 4.1.2. Habitus}

Die unterschiedliche Struktur und der unterschiedliche Umfang der Kapitalsorten sowie die gesamte Sozialisationsgeschichte einer Person vereinen sich im Habitus und zeigen sich in den verschiedenen Lebensstilen. Somit stellt der Begriff des Habitus bei Bourdieu das Bindeglied zwischen den objektiven Lebensbedingungen und dem Verhalten der Personen dar und versteht sich gewissermaßen als inkorporiertes Kapital.

„Die Handlungstheorie, die ich (mit dem Begriff Habitus) vorschlage, besagt letzten Endes, daß die meisten Handlungen der Menschen etwas ganz anderes als die Intention zum Prinzip haben, nämlich erworbenen Dispositionen, die dafür verantwortlich sind, daß man das Handeln als zweckgerichtet interpretieren kann und muß, ohne deshalb von einer bewußten Zweckgerichtetheit als dem Prinzip dieses Handelns ausgehen zu können“" (Bourdieu 1998: 167f).

Für Bourdieu ist der Habitus grundlegend durch eine gewisse Trägheit gegenüber Veränderungen gekennzeichnet. Einerseits ist der Habitus durch seine entwicklungsabhängige und -geschichtliche Determiniertheit von einer gewissen Stabilität, andererseits durch die Dynamik der Zeit einem ständigen Wandel 
unterzogen. Der Habitus ist gleichzeitig Erzeugungs- und Bewertungsinstanz, er ist strukturiertes und gleichzeitig strukturierendes Prinzip. Zum einen ist der Habitus Produkt der Geschichte einer Person, zum anderen beeinflusst und verändert er genau diese Geschichte fortwährend. Entscheidend ist, dass der Habitusbegriff bei Bourdieu über die Bedeutung des Gewohnheitsmäßigen und des Habituellen hinausgeht und eine Art von Eingravierung darstellt (vgl. Bohn 1991: 32ff). So kann beim Habitus einer Person auch von dauerhaften, bis zu einem bestimmten Grad einverleibten Dispositionen gesprochen werden, die fortlaufend das Handeln beeinflussen. Der Habitus ist auch dafür verantwortlich, dass Menschen gleicher oder ähnlicher Soziallagen nahezu identische Formen des Handelns bzw. der Praxis entwickeln (vgl. Winkler 1995: 266). Umgekehrt gesagt bringen aber auch „unterschiedliche Existenzbedingungen unterschiedliche Formen des Habitus“ hervor (Bourdieu 1982: 278). „Wer den Habitus einer Person kennt, der spürt oder weiß intuitiv, welches Verhalten dieser Person verwehrt ist" (Bourdieu 1992a: 33). Er ist damit insbesondere in sozialen Interaktionen und für den Erwerb von symbolischem Kapital von zentraler Bedeutung.

„Wer den kollektiven Erwartungen entspricht, wer nichts zu berechnen braucht, um auf die in einer Situation enthaltenen Befehle unmittelbar eingestellt zu sein, dem fallen alle Profite des Marktes der symbolischen Güter zu. Er hat den Profit der Tugend, aber auch den Profit der leichten Hand, der Eleganz“ (Bourdieu 1998: 174).

Der Habitus kann somit auch als System von Grenzen von Wahrnehmungen, Gedanken, Vorstellungen und Handlungen gesehen werden (vgl. Fröhlich 1994: 38). Beim Habitus handelt es sich somit um „ein strukturiertes System der Wahrnehmungs-, Beurteilungs- und Denkschemata“, durch dessen relative Stabilität „die Akteure in spezifischer Weise ihre Existenzbedingungen kulturell reproduzieren“ (Konietzka 1995: 80). Kurz gesagt kann der Habitus als allgemeine Grundhaltung gegenüber der Welt gesehen werden. Für soziale Interaktionen bedeutet dies: „Soll eine symbolischer Tausch funktionieren, müssen beide Parteien über die annähernd gleichen Wahrnehmungs- und Bewertungskategorien verfügen“" (Bourdieu 1998: 171).

\section{(I) 4.1.3. Feld}

Bourdieu sieht die soziale Welt als mehrdimensionalen Raum, der wiederum aus Teil-Räumen, sogenannten Feldern, besteht, die durch eigene Funktionsgesetze und spezifische Institutionen gekennzeichnet sind (vgl. Bourdieu 1992b: 111). Welche Stellung eine Person innerhalb eines bestimmten Feldes 
einnimmt hängt einerseits davon ab, welche Sorten von Kapital das Feld verlangt und andererseits wie viel der Akteur jeweils von diesen Kapitalien besitzt. Dabei spielt die Zusammensetzung und der Gesamtumfang an Kapital eine Rolle (vgl. Bourdieu 1985 nach Fröhlich 1994: 41). Beispiele für Felder im Sinne Bourdieus: das ökonomische Feld, das politische Feld, das religiöse Feld, das wissenschaftliche Feld, das Feld des modernen Sports, das Feld der Mode, usw. (vgl. Bohn 1991:27). Diese verschiedenen Felder werden wiederum von einem sozialen Raum umfasst, in dem sie in bestimmten Relationen zueinander stehen. Bestimmte Kapitalien, die in einem Feld von großer Bedeutung sind, können in einem anderen Feld sogar hinderlich wirken. Wird jemand in einem Feld tätig, so bestimmt "der Habitus die Präferenzen“ des Akteurs und „das Kapital die Möglichkeiten“ (Winkler 1995: 266). Die in den jeweiligen Feldern als natürlich angesehene soziale Ordnung bezeichnet Bourdieu als Doxa (vgl. Fuchs-Heinritz/König 2005: 202). Bourdieu (1977) pointiert damit die Bedeutung und die Grenzen des Alltagsbewusstseins: „Die Tradition ist schweigsam - schweigt sich vor allem aus über sich als Tradition“"(330).

\section{(I) 4.1.4. Sozialer Raum}

Die Bedeutung der verschiedenen Kapitalsorten und des Habitus zeigt sich in ihrer Funktion bei der Konstruktion des sozialen Raums, in dem Bourdieu die unterschiedlichen sozialen Klassen anhand ihres Kapitalvolumens, ihrer Kapitalstruktur und deren zeitlicher Veränderung lokalisiert (vgl. Bourdieu 1982: 195ff; Gebauer et al. 1999: 34f). Dabei ist zu beachten:

„Bourdieu streicht zwar die grundsätzliche Autonomie von Feldern heraus. Dennoch werden Felder von Strukturen eines übergreifenden Raums relationaler Ungleichheiten mit bestimmt (Bourdieu 2001a[das politische Feld]). Zum Beispiel können Zugangschancen zu einem Feld davon anhängen, welche Positionen Anwärter im sozialen Raum einnehmen“ (Weiß 2004: 213).

Für die vereinfachte Darstellung dieses dreidimensionalen Raums wird dieser auf ein zweidimensionales Koordinatensystem reduziert (vgl. Bourdieu 1982: 212ff). Entlang der y-Achse wird das Kapitalvolumen, welches Bourdieu „als Summe aller effektiv aufwendbaren Ressourcen und Machtpotentiale, also ökonomisches, kulturelles und soziales Kapital“ (Bourdieu 1982: 196) bezeichnet, eingetragen. 
Abbildung 9: Vereinfachte Darstellung des sozialen Raums bei Bourdieu

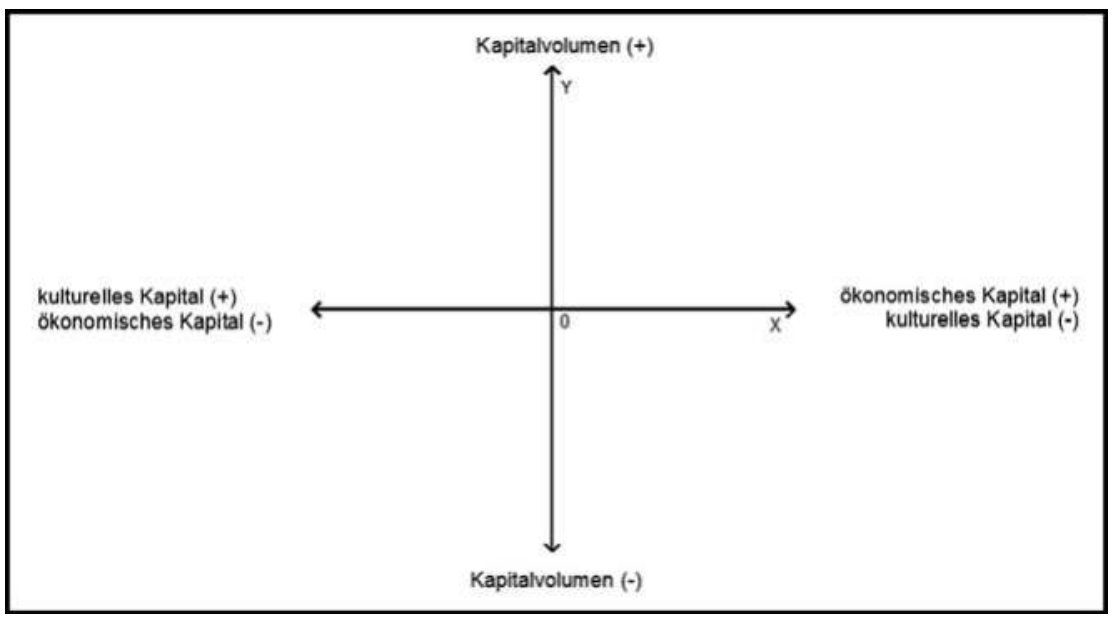

Quelle: vgl. Bourdieu (1982: 212f) in Gebauer et al. (1999: 35).

Den „Umfang der einzelnen Kapitalsorten innerhalb des Gesamtkapitals“ (ebd. 197) kennzeichnet die Kapitalstruktur, welche auf der x-Achse aufgetragen wird. Durch die Dominanz von ökonomischem oder kulturellem Kapital kann es zu einer asymmetrischen Kapitalstruktur kommen (vgl. ebd.). Zum Beispiel verfügen Lehrer über ein relativ hohes kulturelles, aber meist über ein geringes ökonomisches Kapital. Durch diese Unterscheidungen lassen sich bestimmte Berufsgruppen im sozialen Raum verorten. Der Habitus wiederum verbindet die objektiven Klassenpositionen im sozialen Raum mit den symbolischen Formen der Lebensstile (vgl. Gebauer et al. 1999: 36). Dieser Raum der Lebensstile lässt sich wie eine transparente Folie über den sozialen Raum legen (vgl. ebd.).

\section{(I) 4.2. Freiwilligenarbeit und Ehrenamt in der Theorie Pierre Bourdieus}

Betrachtet man die empirischen Studien zur Freiwilligenarbeit mit dem Blick durch die theoretische Brille der Kapital-Habitus-Feldtheorie Pierre Bourdieus (vgl. Bourdieu 1977, 1982, 1983), zeigt sich folgendes Bild: Die Frage nach der Bedeutung der Kapitalien (soziales, ökonomisches, kulturelles Kapital) für Freiwilliges Engagement wird in der Forschung vor allem im Kontext individualistischer Erklärungsansätze von Freiwilligenarbeit behandelt (vgl. Corsten 
et al. 2008). Neben einem breiten Spektrum an meist theorieloser Analyse sozioökonomischer Determinanten bzw. Ressourcen (vgl. Musick/Wilson 2008; Rochester et al. 2010: 42ff; Wilson 2000) stehen vor allem seit den Arbeiten von Robert Putnam $(1993,2000)$ und James Coleman (1988) individuelle (Bourdieu) und kollektive (Putnam, Colemen) Aspekte von „Sozialkapital“ (vgl. Adler/ Kwon 2002: 20; Braun 2001b; Brunie 2009; Franzen/Freitag 2007) im Zentrum der Ursachen- und Wirkungsforschung von Freiwilligem Engagement (vgl. Braun 2007; Brown/Ferris 2007; Kern 2004). Die im Theorieverständnis Bourdieus in ihrem Wechsel- und Zusammenspiel ebenfalls bedeutsamen anderen Kapitalformen (ökonomisches, kulturelles und symbolisches Kapital) (vgl. Bourdieu 2005b: 52), werden zwar teilweise in ihren empirisch messbaren Ausprägungen (z.B. Einkommen, Bildung, Berufsstatus) erfasst, jedoch meist zusammenhanglos im Kanon weiterer sozialstruktureller Merkmale (Geschlecht, Alter, Familienstand, Kinderanzahl), Faktoren bzw. Ressourcen (Erwerbsstatus, u.v.m.) ausgewertet, beschrieben und interpretiert. Sowohl die wechselseitige Transformation wie auch die strukturelle, feldspezifische Bedeutung der Kapitalien bleibt, bis auf wenige Ausnahmen (wie z.B. bei Vogt 2005), in der Freiwilligenforschung größtenteils unberücksichtigt. Zu dem liegt der Schluss nahe, dass durch die einseitige Fokussierung auf Sozialkapital die ökonomischen, kulturellen und vor allem symbolischen Aspekte von Hierarchie und Macht in den Feldern der Freiwilligenarbeit unberücksichtigt bleiben oder aber nicht entsprechend ihrer Bedeutung erfasst werden. Die Frage, $o b$, wo und wie Freiwilligenarbeit als Form alltäglicher Praxis die Klassenstruktur verschleiert, oder aber auch reproduziert, wird nur selten gestellt (vgl. Bourdieu 1982; Solga et al. 2009: 31f). Zuletzt stellt sich auch die Frage, die auf Bourdieus Grundwerk, „Die feinen Unterschiede" verweist. Freiwilligenarbeit als Mittel zur Distinktion? Ob bestimmte Handlungen von der handelnden Person als eher "freiwillig“ oder eher „unfreiwillig“ aufgefasst werden, hängt u.a. von der Klassenzugehörigkeit ab (vgl. Bourdieu 1982: 291). So mag es für einen gesunden Mann mittleren Alters zur sozialen Pflicht gehören, in seinem ländlichen Wohnort der Freiwilligen Feuerwehr und dem Schützenverein anzugehören. Hingegen wird z.B. soziales Engagement in urbanen Zentren vielfach ohne sozialen Druck begonnen und je nach Schichtzugehörigkeit von der jeweiligen Referenzgruppe mit Anerkennung oder Abwehr begegnet. Damit rückt die Freiwilligenarbeit in seinen unterschiedlichen Erscheinungsformen auch in den Blick als „Mittel zur Distinktion“, zu dem sich nicht alle Felder im gleichen Maße eignen dürften. Die generelle Zuschreibung und Kennzeichnung dieser Tätigkeiten als „freiwillig“ verschleiert zum einen deren soziale Bedingtheit und Hintergründe, und zeugt zum anderen vom bürgerlichen Blick der Wissenschaft auf seine Gegenstände. Die 
zunehmende Betonung der „Freiwilligkeit“ gegenüber der „Ehre“ müsste auch vor dem Hintergrund der modernen und postmodernen Individualisierungsdebatte diskutiert werden (vgl. Ehrenberg 2011:472; Nassehi 2008).

Bourdieu selbst hat Freiwilligenarbeit bzw. Ehrenamt nur vereinzelt und am Rande anderer Themen behandelt. Zum Beispiel hat er sich im Rahmen der Analyse der katholischen Kirche mit der internen Arbeitsteilung und der Reproduktion traditioneller Geschlechterrollen am Beispiel des kirchlichen Ehrenamts beschäftigt. Für die Analyse des ungleichen Zugangs zur Freiwilligenarbeit im Allgemeinen sowie zu den Positionen der Freiwilligenarbeit im Besonderen finden sich neben den expliziten Bezügen auch zusätzliche Perspektiven in Bourdieus Arbeiten zu den Themen Ehre, Macht, Anerkennung, Kapitalakkumulation, Distinktion und symbolischen Kapital (vgl. Bourdieu 1987: 205ff; 1998: 161ff).

\section{(I) 4.2.1. Soziale Schließung: Ökonomisches Kapital als Voraussetzung für Freiwilliges Engagement}

In den Forschungsarbeiten zur individuellen Wohltätigkeit (Philanthropie) kann zwischen unterschiedlichen Spendenformen, z.B. der Geldspenden, der Warenspenden, der Blutspenden, etc. unterschieden werden (vgl. Houston 2006). Freiwilligenarbeit wird dabei vorwiegend als Zeitspende, d.h. als Spende von Arbeitszeit betrachtet. ${ }^{27}$ Dass für das Spenden von Geld zumindest ein Minimalausmaß an ökomischen Kapital zur Verfügung stehen muss ist naheliegend. Darüber hinaus bestätigen zahlreiche empirische Studien, dass die Höhe des Einkommens die Spendenhöhe positiv beeinflusst (vgl. Wiepking/Bekkers 2012). Der Besitz von ökomischen Kapital beeinflusst jedoch nicht nur das Spenden von Geld, sondern auch die Möglichkeit zum Spenden von Zeit. Ob man und in welchem Ausmaß man über die eigene Zeit frei verfügen kann wird auch durch die Höhe des verfügbaren ökonomischen Kapitals bestimmt. Dabei wird die Menge der verfügbaren eigenen Zeit durch die „Aneignung der Zeit anderer (in Form von Dienstleistungen) “ und durch unterschiedliche „Formen der Stellvertretung“ gesteigert. Die Möglichkeit dazu hängt jedoch maßgeblich vom Gesamtkapitalvolumen ab (Bourdieu 2005b: 78f). „Umgekehrt werden die Geldersparnisse des Armen mit Zeitverlust bezahlt - das Basteln, die Suche nach Sonderangeboten oder dem günstigen Preis lassen sich nur auf Kosten langer

27 Vergleiche dazu auch die in der Schweizer Freiwilligenforschung verwendete Differenzierung in unterschiedliche Formen der Freiwilligkeit: Geld (Spenden), Arbeitszeit (Freiwillige Arbeit), Naturalien (Material/Infrastruktur) und Prestige (Ideelle Unterstützung, Guter Ruf, Zivilcourage) (Amman 2011: 239). 
Wege, Wartezeiten usw. durchführen" (ebd.). Dabei ist jedoch zu bedenken, dass dem ökonomischen Gesetz von Angebot und Nachfrage folgend, der Wert der Zeit einer Person nicht automatisch mit deren Ausmaß an Verfügbarkeit steigt. Im Gegenteil,

„[d]ie Knappheit, also der Wert, der der Zeit einer Person zuerkannt wird und insbesondere der Zeit, die sie selbst anderen gewährt - die kostbarste, weil persönlichste Gabe (niemand kann sich dabei vertreten lassen) -, bildet eine grundlegende Dimension des soziale Werts dieser Person" (Bourdieu 2001b: 291).

Für Freiwilliges Engagement bedarf es daher einer Mindestmenge an frei verfügbarer Zeit, der jedoch auch ein Wert zugewiesen wird. Ein Zuviel an verfügbarer Zeit, wie dies z.B. bei arbeitslosen Personen der Fall sein kann, entwertet demnach die verfügbare Zeit einer Person und reduziert damit die Höhe an symbolischem Kapital und vielfach auch den Selbstwert. Dies wäre eine plausible Erklärung dafür, dass Arbeitslose in den meisten empirischen Studien die geringsten Beteiligungsquoten an der Freiwilligenarbeit aufweisen.

Freiwilligenarbeit kann nicht nur als Zeitspende, sondern auch als eine Form der Investition in symbolisches und soziales Kapital betrachtet werden (Vogt 2005: 125). Analog zur Akkumulation von kulturellem Kapital bedarf auch die Anhäufung von symbolischem wie sozialem Kapital der Investition von Zeit. Beim Zugang zur Freiwilligenarbeit dürfte damit dem ökomischen Kapital eine ähnliche Bedeutung zukommen wie Bourdieu sie für den Zugang und Verbleib im Bildungssystem beschreibt „D.h. die Umwandlung von ökonomischem in kulturelles Kapital setzt einen Aufwand an Zeit voraus, der durch die Verfügung über ökonomisches Kapital ermöglich wird“ (Bourdieu 1992a: 72). Für Freiwilliges Engagement benötigt man vor allem auch ein über längere Zeit abgesichertes Mindestmaß an ökonomischem Kapital, da die Erzielung von „Investitionsgewinnen“ aufgrund der zum Teil nötigen mehrfachen Konvertierungsvorgänge vergleichsweise langwierig ist. Bei institutionalisiertem kulturellen Kapital wie z.B. in Form von Bildungstitel, können sich die Investitionsprofite bereits relativ zeitnahe am Arbeitsmarkt, z.B. durch höhere Einstiegsgehälter bezahlt machen. Bei der symbolischen Ökonomie hingegen bedarf es längerer Geduld:

„In der Regel muss man länger warten, bis sich eine Investition auszahlt, insbesondere dann, wenn diese Auszahlung wiederum in der nicht-symbolischen Sphäre erfolgen soll, da hier noch Konvertierungsprozesse stattfinden müssen. Symbolische Kapitalkalküle brauchen daher einen langen Atem“" (Vogt 2005: 125).

Die zu erwartenden Gewinne sind darüber hinaus nicht beliebig, sondern von der jeweiligen Gesamtkapitalausstattung abhängig. Bourdieu (2001) beschreibt 
die ungleiche Akkumulation sowie den möglichen Zinseszins von jeglichem Engagement, ähnlich dem bereits beschriebenen Matthäus-Effekt (vgl. DiPrete/ Eirich 2006; Merton 2010), folgendermaßen:

„Das Paradox der Gestreßtheit und Überbeanspruchung der Privilegierten hat darin seine Erklärung: Je mehr wirtschaftliches und kulturelles Kapital, um so mehr Chancen, in den sozialen Spielen zum Erfolg zu kommen, und damit auch mehr Neigung, darin Zeit und Energie zu investieren“ (Bourdieu 2001b: 291).

$\mathrm{Zu}$ erwarten ist, dass die individuellen „Erträge“ der Freiwilligenarbeit nicht in allen Engagementfeldern und für alle Engagierten gleich hoch sind. Zeit würde demnach von den individuellen Akteuren jeweils in jene Felder investiert werden, die den höchsten Gewinn an symbolischem und sozialem Kapital erwarten lassen.

Für die Analyse des ungleichen Zugangs zur Freiwilligenarbeit im Allgemeinen sowie zu den Positionen der Freiwilligenarbeit im Besonderen ist vor allem die jeweils feldspezifische Bedeutung der Kapitalien sowohl inhaltlich als auch methodisch von zentraler Bedeutung. Ehrhardt (2011) sieht ebenfalls den Bedarf, den ökomischen Voraussetzungen für Freiwilliges Engagement vermehrte Aufmerksamkeit zu widmen:

„Auch heute sind zeitintensive ehrenamtliche Tätigkeiten an besondere ökonomische Voraussetzungen gebunden; [...] Dieses Merkmal muss zum einen in Hinblick auf das >allgemeine $<$ Ehrenamt - hier ist insbesondere das traditionelle weibliche Engagement, das durch das Erwerbseinkommen des Ehemannes ermöglicht wird, von Interesse - und zum anderen in Bezug auf Elitepositionen thematisiert werden" (Ehrhardt 2011: 61f).

Für die empirische Analyse des Zusammenhangs von sozialer Ungleichheit und Freiwilligenarbeit stellt sich die Frage, welche Bedeutung dem ökonomischen Kapital beim Zugang zu den Positionen der Freiwilligenarbeit zukommt und in welcher Form dieser Einfluss zwischen den einzelnen Engagementfeldern variiert.

\section{(I) 4.2.2. Hierarchisierung: Interne Arbeitsteilung und die Verschleierung von Ausbeutungsverhältnissen}

Bourdieu hat sich mit der bereits angesprochenen internen Arbeitsteilung und der Reproduktion traditioneller Geschlechterrollen am Beispiel des kirchlichen Ehrenamts beschäftigt. Die typische interne Strukturierung von „katholischen Unternehmen“ beschreibt er pointiert: „[E]inige wenige Geistliche, unterstützt von wenigen bezahlten Kräften, die über eine große Zahl von Ehrenamtlichen verfügen“" (Bourdieu 1998: 190). Die Gründe für den überproportional hohe 
Anteil von Frauen an den Ehrenamtlichen in der Kirche verortet Bourdieu in der klassischen Arbeitsteilung der Geschlechter betreffend der Erwerbsbeteiligung.

„Da Frauen oft mit unbezahlten Tätigkeiten vorliebnehmen müssen und daher weniger in Begriffen der Äquivalenz von Arbeit und Geld denken, sind sie weitaus häufiger als Männer zur insbesondere religiösen und karitativen Ehrenamtlichkeit disponiert" (Bourdieu 2005a: 170).

Frauen übernehmen dabei vielfach den stereotypen Rollenbildern entsprechende Aufgaben, da sich „die - männliche - Priesterschaft [...] beim Einfordern und Annehmen von unentgeltlichen Dienstleistungen auf die herkömmlichen Formen der Arbeitsteilung zwischen den Geschlechtern stützen" kann (Bourdieu 1998: 190ff). Bourdieu ortet in der Kirche und vor allem beim kirchlichen Ehrenamt aufgrund der Ausgestaltung der Produktionsverhältnisse Formen verschleierter Ausbeutung:

„Im religiösen Unternehmen gestalten sich die Produktionsverhältnisse nach dem Modell der Familienbeziehungen: Werden andere Menschen als Brüder behandelt, ist die ökonomische Dimension der Beziehung ausgeklammert. Die religiösen Institutionen arbeiten praktisch wie symbolisch ständig an der Euphemisierung der sozialen Beziehungen einschließlich der Ausbeutungsbeziehungen (wie in der Familie), indem sie sie mittels der Logik des Ehrenamts zu Beziehungen verklären, die auf Geistesverwandtschaft oder religiösen Tausch beruhen [...] Die Ausbeutung wird verschleiert: In den Diskussionen zwischen Bischöfen und Gewerkschaftsvertretern spielen die Bischöfe ständig die Ambivalenz der sakralen Aufgaben aus; sie versuchen, die Gewerkschaftsvertreter zu dem Zugeständnis zu bewegen, daß die geweihten Handlungen selbst Weihe verleihen, daß die religiösen Handlungen Selbstzweck sind und daß, wer sie vollzieht, bereits dadurch belohnt wird, daß er sie vollzieht, daß man sich in der Ordnung der zweckfreien Zweckbestimmtheit befindet“" (Bourdieu 1998: 191).

\section{Dazu nennt Bourdieu auch ein konkretes Beispiel:}

„Auf einer Wallfahrt die Rollstühle der Kranken zu schieben ist ein Akt der Barmherzigkeit, die ein Selbstzweck ist und seinen Lohn im Jenseits hat, und zugleich ein sachlicher Vorgang, der auch von einer bezahlten Krankenschwester ausgeführt werden könnte" (Bourdieu 1998: 191).

Bei säkularen Freiwilligenorganisationen hingegen wird der Lohn der Tätigkeit nicht im Jenseits verortet, sondern durch den Beitrag zum Gemeinwohl ins Diesseits verlagert. Dass trotzdem vielfach religiöse Werte und Normen nicht-religiösen Formen der Freiwilligenarbeit zu Grunde liegen, belegen die zahlreichen empirischen Ergebnisse zum allgemeinen Zusammenhang von Freiwilligenarbeit und Religiosität (vgl. Caputo 2009; Carabain/Bekkers 2011; Taniguchi/Thomas 2011; van Tienen et al. 2011; Vermeer/Scheepers 2012). 
Auch wenn Bourdieu seine Analysen des Ehrenamts vorwiegend auf die katholische Kirche beschränkt hat ist davon auszugehen, dass auch in den anderen Feldern der Freiwilligenarbeit, die Reproduktion von Rollenmustern und sozialer Ungleichheit durch die Naturalisierung der Zugangsregeln sowie der hierarchischen Strukturen verschleiert wird.

\section{(I) 4.2.3. Altruismus und Unbezahlbarkeit als Illusio im Feld der Freiwilligenarbeit}

Dem aktuellen gesellschaftlichen Diskurs liegt vielfach der Altruismus als zentrales Motiv und die Bewertungen der Freiwilligenarbeit als volkswirtschaftlich und gesellschaftlich „unbezahlbares“ Gut zugrunde. Bourdieu (2001) bezeichnet diesen „Glauben an die Sinnhaftigkeit des Spiels in einem bestimmten Feld und an die Bedeutung dessen, was auf dem Spiel steht" (Fuchs-Heinritz/König 2005: 145) als Illusio ${ }^{28}$. Die einem Feld zugrundeliegende Illusio bleibt für die Akteure im Feld implizit und „Außenstehenden mehr oder weniger unverständlich“ (ebd.).

„Als fundamentaler Glaube an den Wert der Diskussionsgegenstände und an die ihrem Diskutieren selbst immanenten Voraussetzungen ist sie [die Illusio] die undiskutierbare Bedingung jeder Diskussion“ (Bourdieu 2001b: 129).

Obwohl unter den Beweggründen für Freiwilliges Engagement Altruismus eines der am häufigsten genannten und diskutierten Motive darstellt, ist die Existenz interessenfreien Handelns vor dem Hintergrund der Überlegungen Bourdieus zur „Ökonomie der symbolischen Güter" in Frage zu stellen. Charakteristisch dafür ist, dass jegliches ökonomische Interesse an den Handlungen verdrängt bzw. zensuriert wird. „Folglich muss die ökonomische Wahrheit, das heißt der Preis, aktiv oder passiv kaschiert werden oder im Ungewissen bleiben. Die Ökonomie der symbolischen Güter [...] beruht auf einem Tabu der expliziten Formulierung" (Bourdieu 1998: 196). Bourdieu folgt daraus weiter, dass dadurch „die für die Ökonomie der symbolischen Güter charakteristischen Strategien und Praktiken immer ambivalent, doppelgesichtig und sogar scheinbar widersprüchlich (beispielsweise haben die Güter in ihr einen Preis und sind »unbezahlbar «) " sind (ebd.). Diese scheinbaren Widersprüche und „Dualität von Wahrheiten, die einander sowohl in den Praktiken als auch im Diskurs (Euphemismus) ausschließen“, sind laut Bourdieu jedoch „nicht als Doppelzüngigkeit

28 Illusio wird laut Fuchs-Heinritz (2005) auch teilweise „als Interesse, involvement bzw. committment übersetzt" (145). 
oder Heuchelei zu denken, sondern als Verneinung, die (gewissermaßen durch "Aufhebung«) für die Vereinbarkeit der Gegensätze sorgt" (ebd.). Als Beispiel für diese Art der Verdrängung und des doppelten Bewusstseins führt Bourdieu (1998) auch das Ehrenamt an.

„Dieser Art von doppeltem Bewußtsein, das wohl allen Akteuren gemeinsam ist, die zugleich dem ökonomischen Universum und einem der anti-ökonomischen SubUniversen angehören (zu denken wäre an Personen, die politisch, gewerkschaftlich usw. aktiv sind, und an alle »Ehrenamtlichen " ", entspringt eine sehr hohe (partielle) Bewußtheit, die [...] bei Personen, die unter keine der üblichen Kategorien fallen und sich daher im Bruch mit den gröberen Selbstverständlichkeiten der Doxa befinden“"vorkommt (Bourdieu 1998: 187).

Er konkretisiert die Ökonomie der symbolischen Güter weiter am Beispiel der Regeln sowie der Illusio und Doxa im Feld der katholischen Kirche:

„Wie in der familialen Ökonomie, von der sie (im dem Modell des brüderlichen Tauschs) eine verklärte Form darstellt, wird auch in der Katholischen Kirche von heute der paradoxe Charakter der Ökonomie der Opfergabe, des Ehrenamts, des Opfers besonders offenkundig: Dieses auf der Verneinung der Ökonomie beruhende Unternehmen mit ökonomischer Dimension ist in der Tat allseits von einem Universum umgeben, in dem mit der Verallgemeinerung des Geldverkehrs das Streben nach der Profitmaximierung zum Prinzip der meisten Alltagspraktiken geworden ist, so daß jeder - religiös oder nicht religiös - Akteur dazu neigt, den Wert seiner Arbeit und seiner Zeit zumindest implizit in Geld zu veranschlagen“" (Bourdieu 1998: 186).

Bourdieu spezifiziert den Vorgang der Verdrängung der ökonomischen Dimension am Beispiel der Funktion des Kirchendieners der „ein mehr oder weniger verdrängter homo oeconomicus" ist weil

„er weiß, daß Blumen auf den Altar zu stellen eine halbe Stunde dauert und daß dies bei Putzfrauentarif soundsoviel einbringt. Gleichzeitig aber spielt er das religiöse Spiel mit und würde die Gleichsetzung seiner gottesdienstlichen Arbeit mit der einer Person aus dem Dienstleistungsgewerbe von sich weisen“" (Bourdieu 1998: 186f).

Dieses doppelte Bewusstsein, wie es Bourdieu für die Kirche beschreibt, trifft in vielen Bereichen auch auf andere Organisationen des Dritten Sektors wie z.B. auf soziale NPOs, Sportvereine etc. zu.

„Die Objektivierung bringt ans Licht, daß die Kirche auch ein Wirtschaftsunternehmen ist; sie läßt aber leicht in Vergessenheit geraten, daß die Kirche ein Wirtschaftsunternehmen ist, das nur so funktionieren kann, wie es funktioniert, weil es nicht wirklich ein Unternehmen ist, weil es sich als Unternehmen verneint. (Genauso wie die Familie nur funktionieren kann, weil sie sich als ein der Definition des Ökonomischen à la Gary Becker gehorchendes Gebilde verneint.)“ (Bourdieu 1998: 186f). 
Bourdieu selbst weitet seine Überlegungen auch auf andere kirchlich-religiöse nicht-gewinnorientierte Bereiche und Organisationen aus:

„Man hat es also mit (schulischen, medizinischen, karitativen usw.) Unternehmen zu tun, die, da sie nach der Logik von Ehrenamt und Opfergabe funktionieren, in der ökonomischen Konkurrenz beträchtlich im Vorteil sind (einer dieser Vorteile: der Markenzeichen-Effekt, gilt doch das Adjektiv christlich als Garantie für eine im Grunde innerfamiliale Moral). Aber solche objektiv ökonomischen Unternehmen können aus diesen Vorteilen nur in dem Umfang Kapital schlagen, wie die Bedingungen des Verkennens ihrer ökonomischen Dimension ständig reproduziert werden, das heißt nur so lange, wie es den Akteuren gelingt, sich und die anderen in dem Glauben zu wiegen, ihre Handlungen hätten keine ökonomische Wirkung" (Bourdieu 1998: 194).

Aktuell wird m.E. genau dieser Aspekt im Kontext von Social Investment, Social Innovation und Social Entrepreneurship diskutiert: Welche Folgen, vor allem im Feld sozialer Dienstleistungen, sind mit der expliziten Verbindung von sozialen mit ökonomischen (gewinnorientierten) Zielen für das Feld und die darin beteilitgen Akteure (inkl. Klienten bzw. Kunden) verbunden? Im aktuellen Diskurs um die Freiwilligenarbeit finden sich zahlreiche, wenn auch nur anekdotische Belege der von Bourdieu beschriebenen „Doppelgesichtigkeit“: So betitelt die Tageszeitung „der Standard“ am 1. Oktober 2012 einen Artikel zur Freiwilligenarbeit mit: „Freiwillige: Unentgeltliche Arbeit ist unbezahlbar"29. Auf der Website zur Freiwilligenarbeit des österreichischen Bundesministeriums für Arbeit, Soziales und Konsumentenschutz (www.freiwilligenweb.at) ist im Vorwort des Bundesministers u.a. zu lesen: „Deshalb müssen wir uns aktiv darum bemühen, dass die

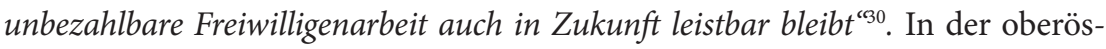
terreichischen Lokalpresse wird der amtierende Landeshauptmann wie folgt zitiert: „Ohne Ehrenamtliche wäre unser Land um vieles ärmer. Ihre Arbeit ist nicht nur unbezahlt, sondern auch unbezahlbar ${ }^{\text {"31 }}$. Auf eine ähnlichen Rhetorik greift auch die damalige Landeshauptfrau (2011) von Salzburg zurück: „Die Tätigkeit der Freiwilligen ist bekanntlich unbezahlbar. Viel wichtiger ist aber, dass sie - auch im übertragenen Sinn - unbezahlbar ist, das heißt unverzichtbar und in ihrer Bedeutung kaum zu überschätzen ${ }^{\text {(32 }}$. Auch freiwillige Vereinigungen bedienen sich

29 http://derstandard.at/1348284632572/Freiwillige-vor-Unentgeltliche-Arbeit-istunbezahlbar - abgerufen am 28.5.2014.

30 http://www.freiwilligenweb.at/index.php?id=CH0524 - abgerufen am 28.5.2014.

31 http://www.meinbezirk.at/linz/leute/unbezahlt-und-unbezahlbar-d117782.html - abgerufen am 28.5.2014.

32 https://www.kommunalnet.at/news/artikel/select_category/7804/article/salzburgschreibt-freiwilligenarbeit-2011-ganz-gross.html?cHash=08e8148f018890f7c6db0da 70a49d937 - abgerufen am 28.5.2014. 
u.a im Rahmen von PR-Kampagnen einer Rhetorik der „Verdrängung des Ökonomischen". Auf einem Plakat der Pfadfinder und Pfadfinderinnen Österreichs ist zu lesen: „Wir machen Kinder- und Jugendarbeit auf höchstem Niveau und ohne

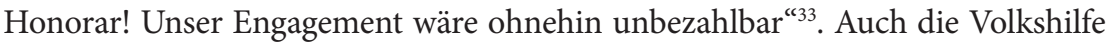
Österreich betitelt das Vorwort in ihrer Ehrenamtsbroschüre mit „Unbezahlt und Unbezahlbar“34 und weiter hinten im Text ist zu lesen: „Die Arbeit, die Freiwillige tagtäglich leisten, ist unbezahlbar und für die Gesellschaft unersetzlich“ (ebd.: 6). Ähnliche Slogans finden sich auch bei zahlreichen weiteren Nonprofit Organisationen u.a. aus den Bereichen der Sozialen Dienste, der Katastrophenhilfs- und Rettungsdienste sowie des Sports. Damit wird auch deutlich, welche Legitimitätsfunktion Freiwilligenarbeit für Nonprofit Organisationen hat. Freiwilligenarbeit trägt entscheidend zur Verkennung der ökonomischen Dimension von Freiwilligenorganisationen, z.B. im Feld des Sports oder der Religion, bei. Es wird sich zeigen, ob sich jene Organisationen die sich als Social Business bezeichnen, d.h. die Soziales mit Ökonomischem explizit verbinden, künftig auch der legitimitätsgenerierenden Funktion der Freiwilligenarbeit bedienen werden. Denn für die Gesamtheit der Freiwilligenarbeit trifft m.E. das zu, was Bourdieu bisher nur als Illusio für das religiöse Feld konkret beschrieben hat. Nämlich,

„daß es geradezu zu den Voraussetzungen des Funktionierens und des Erfolgs des religiösen Unternehmens gehört, daß die religiösen Akteure an das glauben, was sie tun, und die streng ökonomische Interpretation ihres Handelns und ihrer Funktion von sich weisen“" (Bourdieu 1998:189).

Sowohl in Bezug auf aktuell diskutierte Formen sozialer und ökonomischer Verschneidungen als auch betreffend der politisch attestierten, sozialintegrativen bzw. inklusiven Potentiale der Freiwilligenarbeit, sind die Organisationen bzw. das jeweilige Feld gefordert:

„Man steht hier wieder vor dem bereits bekannten Problem, das sich bei der expliziten Formulierung der Wahrheit von Institutionen (oder von Feldern) stellt, deren Wahrheit ist, daß sie die explizite Formulierung ihrer Wahrheit verweigern. Einfacher gesagt: Wenn die ganze Logik des explizit formulierten Universums auf dem Tabu der expliziten Formulierung beruht, bewirkt die explizite Formulierung eine destruktive Veränderung" (Bourdieu 1998: 186).

33 https://www.ppoe.at/aktionen/bundesthema/bundesthema1011/pdf/faq_poster_ejf_ rgb.pdf - abgerufen am 28.5.2014.

34 http://www.volkshilfe.at/ehrenamt?gclid=CKu81pz9hLsCFQ1L3god6A4AzA - abgerufen am 28.5.2014. 
Eine der Wahrheiten, der sich die Freiwilligenarbeit verweigern muss, ist auch, dass nicht alle Gesellschaftsmitglieder in gleichem Maße erwünscht sind, nicht jeder ungeachtet seiner Kapitalausstattung, seines Habitus und seines Lebensstils gebraucht wird und damit nicht jedem Freiwilligenarbeit als potentielle Quelle für soziale Anerkennung, soziale Integration, Kompetenzerwerb, Freizeitzeitgestaltung oder aber Selbstverwirklichung offen steht. Dies wäre zumindest eine Erklärung dafür, warum der sozial ungleiche Zugang zu Freiwilligenarbeit seit Beginn der Engagement- und Partizipationsforschung dokumentiert ist, sich an den Phantasien zum Integrations- bzw. Inklusionspotential der Freiwilligenarbeit in der öffentlichen wie politischen Diskussion jedoch nur wenig geändert hat. Es mag auch erklären, warum die Freiwilligenorganisationen selbst bis heute die unterschiedlichen Formen der Profite der Freiwilligenarbeit für die Freiwilligen selbst verneinen, und stattdessen die Selbstlosigkeit und Aufopferung ihrer Freiwilligen, d.h. das Altruismus-Motiv, hervorstreichen. Dies betrifft im besonderen Maße Leitungs- und Führungspositionen in der Freiwilligenarbeit.

„In diesem Zusammenhang kann man darüber hinaus mit Barlösius (2005) fragen, ob diese Form der Erzeugung und Repräsentation sozialer Ungleichheit nicht auch besonders folgenreich ist, da die ungleichen Zugangsmöglichkeiten zu Positionen mit Entscheidungsbefugnissen beim ehrenamtlichen Engagement nicht problematisiert werden. Die Verbindung von wirtschaftlicher und politischer Macht wird hier mit dem Etikett der Selbstlosigkeit versehen“ (Ehrhardt 2011: 64).

Mit der Verneinung der ökomischen Dimension gerät auch aus dem Blick, dass, wie in allen anderen gesellschaftlichen Feldern auch, in der Freiwilligenarbeit unterschiedliche Kapitalformen akkumuliert werden können und dies infolge zur (Re-)Produktion sozialer Ungleichheit beiträgt.

\section{(I) 4.2.4. Der Lohn der Freiwilligenarbeit: Die ungleiche Verteilung von Anerkennung und Ehre}

Wie eingangs erwähnt, hängt der Wert der (ökonomischen, sozialen, kulturellen, etc.) Kapitalien zentral von deren (feldspezifischen) Anerkennung ab, d.h. von den Ergebnissen der (sozialen) Kämpfe um die Vormachtstellung bestimmter Kapitalien (vgl. Weiß 2004: 212). Diese „Kämpfe um Anerkennung“ bilden für Bourdieu (1992b) „eine fundamentale Dimension des sozialen Lebens“ (37). Dabei geht es um die "Akkumulation einer besonderen Art von Kapital [...] - eben »Ehre« im Sinne von Reputation, Prestige“, um „auf Bekanntheit und Anerkennung begründetem Kapital“ (ebd.). Anerkennung wird, im Kontext der Koordination bzw. des Managements von Ehrenamtlichen, als zentraler Faktor angesehen, wenn es um Fragen der Motivation, des Haltens und der 
immateriellen Entlohnung von Freiwilligen geht (vgl. Schlesinger/Nagel 2013). (Soziale) Anerkennung wird damit von Seiten der Freiwilligenorganisationen, als auch von Seiten der Politik, als die primäre Gegenleistung für Freiwilliges Engagement konstruiert und gefordert. Bourdieu (2001b) sieht jedoch gerade auch in der ungleichen Verteilung von Anerkennung und Prestige eine der zentralen Dimensionen sozialer Ungleichheit.

„Die soziale Welt vergibt das seltenste Gut überhaupt: Anerkennung, Ansehen, das heißt ganz einfach Daseinsberechtigung. Sie ist imstande, dem Leben Sinn zu verleihen, und, indem sie ihn zum höchsten Opfer weiht, selbst noch dem Tod. Weniges ist so ungleich und wohl nichts grausamer verteilt als das symbolische Kapital, das heißt die soziale Bedeutung und die Lebensberechtigung" (Bourdieu 2001b: 309f).

Der sozial ungleiche Zugang zur Freiwilligenarbeit führt damit zu einer ungleichen Akkumulation von symbolischem Kapital und hat Auswirkung auf die Handlungschancen und -optionen der einzelnen Individuen. Es ist anzunehmen, dass nicht alle Felder der Freiwilligenarbeit in gleichem Maße symbolisches Kapital und Prestige, d.h. gesellschaftliches Ansehen vermitteln (vgl. Künemund/ Schupp 2007: 1). Somit dürfte die ungleiche Verteilung von Anerkennung, d.h. von symbolischem Kapital, eine der zentralen Ungleichheitsdimensionen sein, die durch die Freiwilligenarbeit reproduziert werden. Da mit sozialer Anerkennung, in Abhängigkeit des jeweiligen Feldes, auch Ansehen und Prestige in anderen Feldern verbunden sein kann, wird damit auch gesamtgesellschaftlich Macht und Einfluss ungleich verteilt. Bourdieu beschreibt m.E. einen für die Individuen zentralen Aspekt der Auswirkungen ungleicher Machtverteilung.

„Je mehr Macht man über die Welt hat, umso mehr passen die Aspirationen zu ihren Realisierungschancen, um so vernünftiger, stabiler und gegenüber symbolischer Manipulation unempfindlicher sind sie. Unterhalb einer bestimmten Schwelle dagegen sind Aspirationen unstet, von der Realität abgekoppelt und zuweilen wie verrückt, so als würde dann, wenn nichts wirklich möglich ist, alles möglich“ (Bourdieu 2001b: 290).

Es gilt daher Freiwilligenarbeit auch als Ort der Machtausübung und Machtakkumulation zu denken. Insbesondere bei ehrenamtlichen Funktionen in Leitungsgremien (Vorständen, Komitees, etc.) aber auch bei allen anderen Positionen mit Leitungs- und Führungsfunktionen ist der ungleiche Zugang für die Reproduktion von Handlungsspielräumen und Gestaltungsmacht von Bedeutung.

Nach einem Streifzug durch die Überlegungen von und mit Bourdieu zur Freiwilligenarbeit wird im nachfolgenden Kapital der Versuch unternommen, die bisher beschriebenen und diskutierten Perspektiven auf soziale Ungleichheit und Freiwilligenarbeit in einem Modell der (Re-)produktion sozialer Ungleichheit zusammenzuführen. 


\section{(I) 5. Fazit: (Re)-Produktion sozialer Ungleichheiten in der Freiwilligenarbeit durch Soziale Schließung und Hierarchisierung}

Für die Analyse von Gesellschaftsbereiche die bislang nicht oder nur randständig Gegenstand der Ungleichheitsforschung gewesen sind, stellt folgende, auf dem theoretischen Ansatz Bourdieus fußende Perspektive einen vielversprechenden empirischen Forschungsansatz dar, der auf bestehende Daten angewendet werden kann: Freiwilligenarbeit wird dabei nicht als neue Ungleichheitsdimension konstruiert, sondern die Felder, in denen Freiwilligenarbeit geleistet wird, werden als Orte der Reproduktion bzw. Genese sozialer Ungleichheit (vgl. Bourdieu 1992b; Diewald/ Faist 2011) verstanden. Diese Perspektive erscheint nicht zuletzt auch im Hinblick einer Integration des Dritten Sektors in die Analysen und Erklärungsmodelle sozialer Ungleichheit als fruchtbringender. Nachfolgendes Modell zur Reproduktion sozialer Ungleichheit in der Freiwilligenarbeit (Abbildung 10) versucht unter Bedachtnahme der Theorie Pierre Bourdieus, die Perspektiven zu den Determinanten, Dimensionen und Auswirkungen mit der Perspektive, Freiwilligenarbeit als Kontextebenen der Genese sozialer Ungleichheit zu verstehen, zusammenzuführen.

In der Freiwilligenarbeit kommt es auf Basis sozialer Mechanismen wie der sozialen Schließung, der Hierarisierung und in Teilbereichen auch der Ausbeutung unter dem direkten wie auch moderierenden Einfluss der zentralen askriptiven (Heterogenitäts-)Merkmale (Geschlecht, Alter, Herkunft, etc.) zur Reproduktion bestimmter Ungleichheitsdimensionen, wie jener von gesellschaftlicher Teilhabe (allgemeiner Zugang zur Freiwilligenarbeit) sowie von Macht und Prestige durch hierarchische Organisation (Position in der Freiwilligenarbeit). Dies kann mit einer ungleichen Akkumulation von symbolischen, sozialen und kulturellen $\mathrm{Ka}$ pitalien (Bourdieu 1983) verbunden sein und so ihrerseits zu ungleichen Handlungsmöglichkeiten in anderen Dimensionen führen, diese verstärken oder auch verringern. Als Auswirkungen der Reproduktion sind eine generell höhere Lebenszufriedenheit, eine besserer Gesundheitszustand sowie der Erwerb von jobrelated skills (Wilson/Musick 1999) und eine mögliche ability signaling Funktion miteinzubeziehen. Aus dem Stand der Forschung sowie aus den ungleichheitstheoretischen Überlegungen folgt somit, dass sich hinter dem ungleichen Zugang zur Freiwilligenarbeit mehr als nur soziale Differenzierung, Heterogenität oder Verschiedenartigkeit vermuten lässt. 


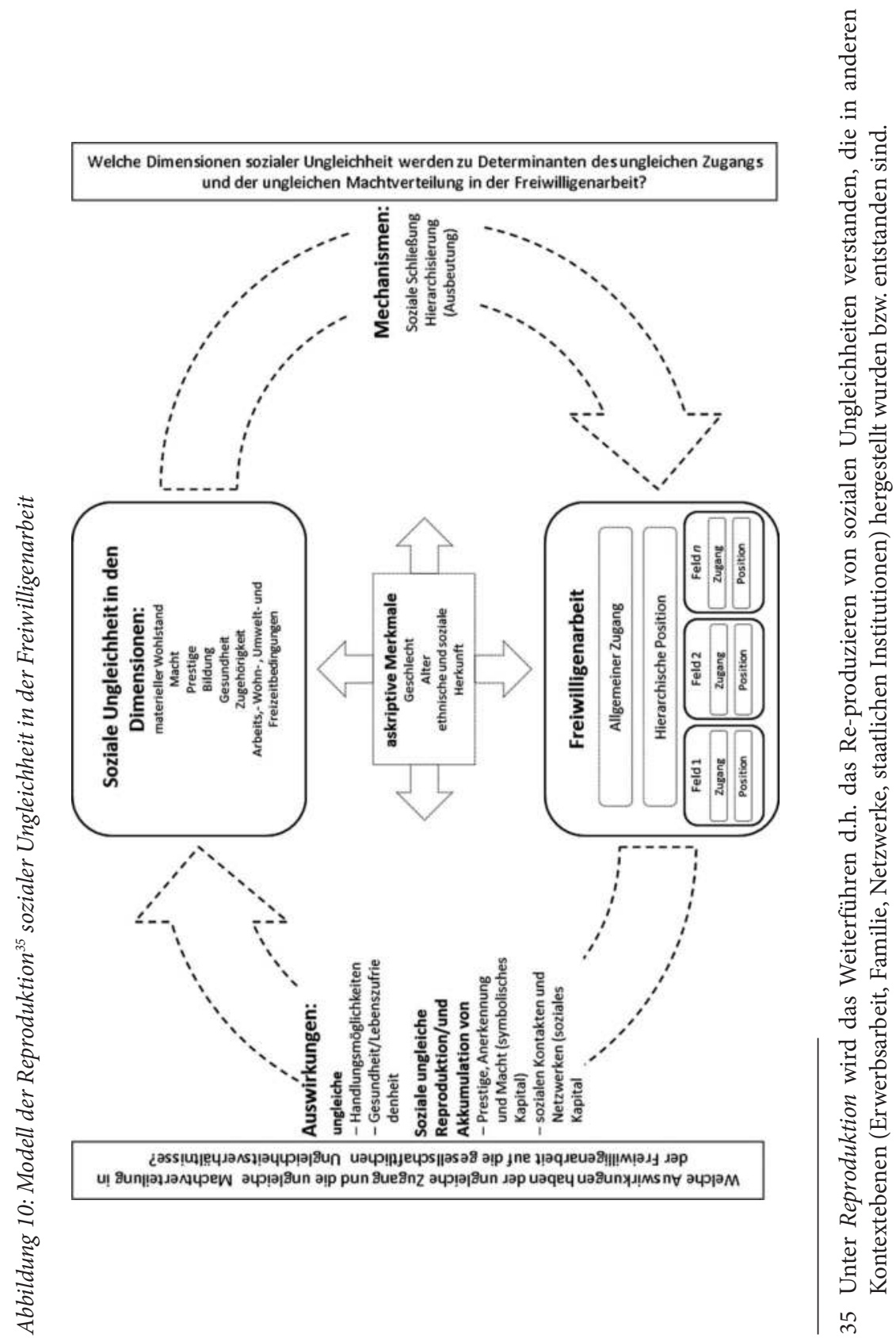


Mit der Reproduktion sozialer Ungleichheiten ist die Kumulation „sich gleichende[r] Ungleichbehandlungen aufgrund eines bestimmten Heterogenitätsmerkmals in verschiedenen Lebensbereichen bzw. Kontexten (z.B. Erwerbsarbeit und Haushalt)“ (Diewald/Faist 2011: 106) zu verstehen. Auf der einen Seite ist denkbar, dass sich die Ungleichbehandlung aufgrund eines bestimmten Merkmals auch in der Freiwilligenarbeit fortsetzt, sei es in Form eines eingeschränkten Zugangs im Allgemeinen oder aber zu bestimmen Positionen im Besonderen. Hierbei ist primär an askriptive Merkmale wie das Geschlecht, das Alter, die ethnische Herkunft zu denken. Weiters, ob und in welcher Form eine Ungleichbehandlung z.B. auf der Kontextebene formaler Organisationszugehörigkeit in der Erwerbsarbeit, auch in der Freiwilligenarbeit zu einer Ungleichbehandlung im Sinne einer Zugangsbarriere, sei es in Form von Fremd- oder Selbstselektion, führt. Ein Bespiel dafür wäre die geschlechterbezogene Ungleichbehandlung beim Zugang zu Führungspositionen in der Erwerbsarbeit und in der Freiwilligenarbeit (vgl. Rotolo/Wilson 2007). Ein weiterer Fall ist die Kopplung des Zugangs zur Freiwilligenarbeit an den Zugang zur Erwerbsarbeit. Eine andere Form der Reproduktion wäre die „immer wiederkehrende Ungleichbehandlungen in aufeinanderfolgenden Lebensphasen bzw. institutionell verknüpften Kontexten (z.B. Ausbildung, Beruf)“ (Diewald/Faist 2011: 106). Hier sei auf die bestehende Forschung zur „Freiwilligenarbeit im Alter“ verwiesen, die zeigt, dass vielfach mit einem Ausstieg aus der Erwerbsarbeit (Pensionierung) auch ein Ausstieg aus der Freiwilligenarbeit verbunden ist (vgl. Erlinghagen 2007; Erlinghagen et al. 2006). Hier stellt sich die Frage, ob diese beobachtbare Doppelexklusion alle Personengruppen gleichermaßen betrifft, oder ob hier nach sozialen Merkmalen strukturierende Effekte auftreten, z.B. dass diese Doppelexklusion vor allem Personen mit geringem Bildungsgrad und gesundheitlichen (psychisch wie physischen) Beeinträchtigungen betrifft.

Wie der Stand der Forschung (siehe Kapital II) zeigt, sind darüber hinaus zahlreiche statusrelevante erworbene Merkmale (Kapitalien) für den Zugang zu den Feldern und Positionen von Bedeutung. Diese gilt es, für den ungleichen Zugang zur Freiwilligenarbeit in besonderem Maße in den Blick zu nehmen. Bei der Frage nach der Reproduktion von Ungleichheitsdimensionen ist vordergründig an jene zu denken, die in der Freiwilligenarbeit auch akkumuliert werden können. Aus der Liste der Ungleichheitsdimensionen (vgl. Hradil 2005; Kreckel 2004; Solga et al. 2009) (materieller Wohlstand, Prestige, Macht, Bildung, Gesundheit, gesellschaftlichen Teilhabe, etc.) ist vor allem an die Reproduktion des Zugangs zu hierarchischen Positionen, die mit Prestige (symbolisches Kapital), Anerkennung und Macht verbunden sind, dem Zugang zu bedeutsamen sozialen Netzwerken (soziales Kapital) sowie generell der gesellschaftlichen Teilhabe und der 
damit verbundenen Handlungsmöglichkeiten zu denken. Diese Dimensionen reproduzieren sich primär über die Mechanismen der sozialen Schließung (selektiven Assoziation), der hierarchischen Organisation sowie möglichweise auch über verschleierte Formen der Ausbeutung. In diesen Fällen sind der sozial selektive Zugang zur Freiwilligenarbeit und die Zuweisung von Positionen innerhalb der Freiwilligenorganisation entscheidend. Ähnliches wie es Kreckel (2004) für die Arbeitswelt beschreibt, ist auch für die Freiwilligenarbeit anzunehmen:

„Die Handlungsspielräume und -befugnisse eines Berufstätigen ebenso wie sein Einkommen sind somit zunehmend von seiner Stellung innerhalb der ihn beschäftigenden hierarchischen Organisation (sowie von deren Qualität und Gewicht im Konzert der Organisationen) abhängig, nicht unmittelbar von seinen persönlichen Leistungen und Fähigkeiten: Ohne eine berufliche Position oder ein »Amt « läßt sich in der Arbeitswelt ebensowenig etwas bewirken wie in der Politik; zuerst wird die Position, erst dann die Person ernstgenommen“ (Kreckel 2004: 73)

Darüber hinaus ist in Abhängigkeit des jeweiligen Feldes und der konkreten Tätigkeit auch die Reproduktion von ungleichem Gesundheitsstatus oder ungleicher Kompetenzen (unterschiedliche Formen von kulturellem Kapital) denkbar. Die direkte Reproduktion von Wohlstandsfaktoren (ökonomisches Kapital) ist in der Freiwilligenarbeit per Definition sowie durch die Illusio des Feldes ausgeschlossen. Aus einer längerfristigen Perspektive ist die zeitlich verzögerte teilweise Rücktransformation des in Form von arbeitsfreier Zeit indirekt investierten ökonomischen Kapitals und die Transformation des akkumulierten symbolischen Kapitals (Prestige und Macht) sowie des sozialen Kapitals (z.B. Weak Ties) in ökonomisches Kapital sehr wahrscheinlich (Bourdieu 1977, 1983).

Der vielfach geteilten stereotypen Annahme zum integrativen Potential der Freiwilligenarbeit folgend ist jedoch auch die Frage zu stellen, „[h]eben sich die Ungleichheiten wechselseitig auf oder wirken sie so zusammen, dass sich strukturierte soziale Ungleichheiten herausbilden?“ (Weiß 2004: 208). Soziale Ungleichheiten können dabei auch in Konkurrenz zueinander stehen, konfliktär oder aber auch substituär sein (vgl. Diewald/Faist 2011: 106). Es ist daher durchaus denkbar, dass die, in anderen Feldern produzierten Ungleichheiten durch die Freiwilligenarbeit wieder reduziert werden und es damit zu einer Substitution von Ungleichhandlungen im Feld der Erwerbsarbeit, der Familie oder der staatlichen Institutionen kommt. Es gilt daher auch empirisch zu prüfen ob und in welchen Bereichen bzw. Subfeldern der Freiwilligenarbeit, Ungleichheitsbehandlungen aus anderen Kontextebenen und Feldern kompensieren und damit in Summe reduzieren oder aufgehoben werden (z.B. die Inklusionen und infolge Reintegration von Arbeitslosen in das Beschäftigungssystem). Aus dieser Perspektive erscheint es sinnvoll und notwendig, die Freiwilligenarbeit in den 
jeweils unterschiedlichen Bereichen und Feldern getrennt zu untersuchen. Es ist zu erwarten, dass es z.B. im Feld der sozialen Dienste und im Feld des Sports zu je unterschiedlichen Ausprägungen der Reproduktion oder auch Reduktion sozialer Ungleichheit kommt.

Die vorliegende Arbeit versteht sich dabei nicht primär als Beitrag zur Theoriebildung sozialer Ungleichheit, sondern als Analysefolie von bisher von gesellschaftskritischen Perspektiven ausgeklammerten Handlungsfeldern des Dritten Sektors. Trotz dieses fehlenden Anspruches sind die in Kapitel (III) folgenden empirischen Ergebnisse nicht unbedeutend für die Ungleichheitsforschung, denn diese benötigt um "gesellschaftstheoretisch und gesellschaftspolitisch bedeutsam zu sein, [...] Kenntnis [...] über die Regeln, nach denen Ungleichheiten in verschiedenen Teilsystemen produziert werden, und in welcher zeitlichen Ordnung und welchen Interdependenzverhältnisse diese zueinander stehen“ (Diewald/Faist 2011: 93). Bevor jedoch die empirischen Analysen zur Freiwilligenarbeit in Österreich in Kapitel (III) vorgestellt und diskutiert werden, erfolgt im nächsten Kapitel die Darstellung des aktuellen internationalen Forschungsstandes zu den Determinanten und Auswirkungen der Freiwilligenarbeit. 


\section{(II) Stand der Forschung zu den Determinanten und Auswirkungen der Freiwilligenarbeit}

Dieses Kapitel fasst den Stand der Forschung zum sozial ungleichen Zugang zur Freiwilligenarbeit sowie den Auswirkungen der Freiwilligenarbeit auf die freiwillig Tätigen zusammen. Zu Beginn wird, zur Verortung des Ungleichheitsthemas innerhalb der Freiwilligenforschung, ein kurzer Überblick zur aktuellen Engagement- und Partizipationsforschung im Allgemeinen und $\mathrm{zu}$ den kritischen Perspektiven auf die Freiwilligenarbeit im Besonderen gegeben.

\section{(II) 1. Aktuelle Forschungsstränge}

Als interdisziplinäres Forschungsfeld beschäftigen sich die Ökonomie, Psychologie, Soziologie, Politikwissenschaften und Teile der Wirtschaftswissenschaften sowie zunehmend Disziplinen wie die Gerontologie und die Gesundheitswissenschaften mit Freiwilligem Engagement. In Folge des gewachsenen Interesses hat sich auch die Anzahl der Publikationen zum Themengebiet der Freiwilligenarbeit (engl. „volunteering“ und „voluntary work“) von den 1980er bis zum Ende der 2000er Jahren beinahe verzwanzigfacht ${ }^{36}$. Entsprechend ausdifferenziert sind mittlerweile die Themengebiete. Hustinx et al. (2010) bieten einen umfassenden Überblick zu den im Kontext der Engagement- und Partizipationsforschung behandelten Fragestellungen und theoretischen Zugängen (siehe Abbildung 11).

36 Die Suche nach den Begriffen „volunteering“ und „voluntary work“ bei ProQuest, einem us-amerikanischen Host für Internetpublikationen (http://search.proquest.com), liefert für die 1980er Jahr (1980 bis 1989) 1.492 Peer reviewed Artikel. Für den Zeitraum von 1990 bis 1999 beträgt die Anzahl bereits 10.551 Artikel und für die 2000er Jahre (2000 bis 2009) 28.522 Artikel (abgerufen am 27.3.2014). 
Abbildung 11: A hybrid conceptual framework of volunteering

\begin{tabular}{|c|c|c|}
\hline $\begin{array}{l}\text { Layers of } \\
\text { complexity }\end{array}$ & Theoretical building blocks & Key frameworks and approaches \\
\hline $\begin{array}{l}\text { The problem of } \\
\text { definition }\end{array}$ & What do we study? & $\begin{array}{l}\text {-Defining what volunteering is not } \\
\text {-Defining what volunteering is } \\
\text {-Volunteering as a social construct }\end{array}$ \\
\hline $\begin{array}{l}\text { The problem of } \\
\text { multidisciplinarity }\end{array}$ & Why do we study it? & $\begin{array}{l}\text {-Economists: impure altruism } \\
\text {-Sociologists: social cohesion and } \\
\text { social welfare } \\
\text {-Psychologists: prosocial } \\
\text { personality } \\
\text {-Political scientists: citizenship and } \\
\text { democracy }\end{array}$ \\
\hline \multirow[t]{3}{*}{$\begin{array}{l}\text { The problem } \\
\text { of theory as } \\
\text { multidimensional }\end{array}$} & $\begin{array}{l}\text { Theory as explanation: } \\
\text {-Why do people volunteer } \\
\text {-Determinants of } \\
\text { volunteering }\end{array}$ & $\begin{array}{l}\text {-Motivations and benefits } \\
\text {-Dominant status model } \\
\text {-Resource model } \\
\text {-Theories of cross-national } \\
\text { variation in volunteering }\end{array}$ \\
\hline & $\begin{array}{l}\text { Theory as a narrative } \\
\text {-How do people volunteer } \\
\text { - The context of volunteering } \\
\text {-Volunteering and social } \\
\text { change }\end{array}$ & $\begin{array}{l}\text {-Styles of volunteering } \\
\text {-The volunteer process } \\
\text {-The volunteer ecology } \\
\text {-Volunteer management } \\
\text {-The changing institutional and } \\
\text { biographical embedding of } \\
\text { volunteering }\end{array}$ \\
\hline & $\begin{array}{l}\text { Theory as enlightenment } \\
\text {-Critical perspectives }\end{array}$ & $\begin{array}{l}\text {-Issues of social inequality } \\
\text {-Negative consequences of } \\
\text { volunteering } \\
\text {-Unmet expectations } \\
\text {-Hidden ideologies }\end{array}$ \\
\hline
\end{tabular}

Quelle: Hustinx et al. (2010a: 413).

Die Forschungsbereiche beschäftigen sich dabei mit grundlegenden Fragen der Begriffsdefinition (vgl. Cnaan et al. 1996; Smith 1975), mit dem Einfluss von sozio-ökonomischen und sozio-kulturellen Determinanten (vgl. Wilson 2012) sowie dem Einfluss der sozialen Herkunft und Formen der intergenerationalen Übertragung von Freiwilligem Engagement (vgl. Bekkers 2007; Caputo 2009; Suanet et al. 2009). Auch sozialpsychologische Fragen nach den Ursprüngen und Bedingungen prosozialen Verhaltens (vgl. Lichter et al. 2002; Penner et al. 2005) und Fragen des Zusammenhangs von sozialer Kohäsion, Wohlfahrtsstaatmodellen, Demokratie und gesellschaftlicher Partizipation werden 
untersucht und erörtert (vgl. Parboteeah et al. 2004; Stadelmann-Steffen 2011). Ein weiterer Bereich untersucht den Wandel der Freiwilligenarbeit und die Ausgestaltungen und die Verbreitung neuer Engagementformen (vgl. Hustinx 2010; Hustinx/Lammertyn 2003). Ergänzend finden sich Arbeiten, die den Einfluss der Gene bei der intergenerationalen Übertragung der Freiwilligenarbeit untersuchen und so Disziplinen wie die Genetik und Epigenetik mit der Freiwilligenforschung verbinden (vgl. Son/Wilson 2010). Auch die ökonomische Bewertung der Freiwilligenarbeit (vgl. Brown 1999; Salamon et al. 2011; Wolozin 1975) gewinnt im Zuge der Managerialisierung von Nonprofit Organisationen (vgl. Maier/Meyer 2011; Meyer et al. 2013) und dem Trend zur Wirkungsmessung im Dritten Sektor (z.B. Social Impact Assessment, Social Return on Investment, vgl. Maier et al. 2014) an Bedeutung (vgl. Moxham/ Boaden 2007).

Die Forschungsarbeiten, die sich kritisch mit der Freiwilligenarbeit bzw. generell den verschiedenen Formen zivilgesellschaftlicher Partizipation beschäftigen, können grob zu vier Themenbereichen zusammengefasst werden (siehe Abbildung 11 - critical perspectives). Studien und Theorien, die sich (1) mit Fragen sozialer Ungleichheit beschäftigen, die (2) negative Konsequenzen der Freiwilligenarbeit erforschen, die (3) nicht erfüllbare Erwartungen thematisieren, und zuletzt Arbeiten, die (4) versteckte Ideologien in der Freiwilligenarbeit aufdecken (vgl. Hustinx et al. 2010a: 413, 427f).

Bei konkreter Recherche finden sich hingegen nur wenige Studien, die sich explizit mit dem Zusammenhang von Freiwilligenarbeit und sozialer Ungleichheit beschäftigen (vgl. z.B. Heitzmann et al. 2009; Institute for Volunteering Research 2004; Rotolo et al. 2010). Eine längere Tradition kritischer Arbeiten gibt es im Bereich der politischen Partizipation- und Engagementforschung. Seit den Arbeiten von Verba et al. (1995) mit dem Titel „Voice and Equality“ und den Studien von Putnam $(1993,1995)$ zur gesellschaftlichen Bedeutung von Sozialkapital ${ }^{37}$ stehen die Zusammenhänge zwischen politischer und zivilgesellschaftlicher Partizipation mit sozialer Ungleichheit im Fokus der kritischen Zivilgesellschafts- und Partizipationsforschung (vgl. Bödeker 2012; Brady 2003; Caínzos/Voces 2010; Heitzmann et al. 2009; Uslaner/Brown 2008; Walter 2012). Solt (2008) untersuchte in diesem Zusammenhang den Einfluss von Einkommensungleichheit auf unterschiedliche Formen demokratischer politischer Partizipation (Political Interest, Political Discussion, Elecctoral Participation): Eine höhere Einkommensungleichheit wirkt sich dabei vor allem

37 zur Begriffsklärung und Konzeptabgrenzung siehe Coffé/Geys (2007), Brunie (2009). 
negativ auf die politische Beteiligung der unteren Einkommensschichten aus und führt in Folge zu einer Zunahme an partizipatorischer Ungleichheit sowie ungleicher Machtverteilung in der Gesellschaft. Darüber hinaus ist die (kritische) Partizipationsforschung traditionell eng mit der Sozialkapitalforschung verbunden (vgl. Braun 2003a; Brown/Ferris 2007; Brunie 2009; Coffé/Geys 2007; Iglic 2010; Putnam 2000; Stolle/Hooghe 2005; van Deth 2010). Weitere Arbeiten beschäftigen sich z.B. mit den Auswirkungen der unterschiedlichen Formen von Sozialkapital (bridging social capital versus bonding social capital) und der „Sozialen Ordnung in Freiwilligenvereinigungen“ (vgl. Nagel 2003a). Die Erkenntnisse der (sozialen) Netzwerkforschung zur sozialen Homogenität, zur Gendersegregation (vgl. Messner/Bozada-Deas 2009; Peter/Drobnič 2013; Popielarz 1999; Rotolo/Wilson 2007) und zu den selektiven Wirkungen von Homophilie (vgl. McPherson et al. 2001) stellen das soziale Integrationspotential bzw. die brückenbildende Funktion von Freiwilligenorganisationen ebenfalls in Frage.

Die Analyse des Zusammenhangs von Freiwilligenarbeit und sozialer Ungleichheit berührt dabei die von Hustinx et al. (2010a) unter den kritischen Perspektiven zusammengefassten Themen der negativen Konsequenzen, versteckten Ideologien und nichterfüllbaren Erwartungen der Freiwilligenarbeit. Auch für die basale Frage nach dem Warum („Why do people volunteer?"), d.h. nach den Voraussetzungen und Determinanten der Freiwilligenarbeit liefert die vorliegende Arbeit weitere Antworten. Die Arbeit stellt praxisrelevante Erkenntnisse für das (Personal-)Management von NPOs hinsichtlich der Rekrutierung und der Koordination von Freiwilligen bereit. Der ungleichheitstheoretische Zugang hat zum Ziel, für Themen des Diversitätsmanagements zu sensibilisieren und zu einem kritischen Blick auf die sozialen Wirkungen bewusster wie unbewusster Selektionsmechanismen anzuregen.

\section{(II) 2. Einflussfaktoren auf Freiwilligenarbeit}

Die Einflussfaktoren die formelle Freiwilligenarbeit prägen, können auf unterschiedlichen Kontextebenen verortet werden (siehe Abbildung 12). Auf der Ebene des gesellschaftlich-kulturellen Kontextes, des aktuellen sozialen Kontextes, des Herkunftskontextes sowie auf Ebene der Person (vgl. Meyer/Rameder 2011; More-Hollerweger/Rameder 2013). 
Abbildung 12: Einflussfaktoren auf Freiwilliges Engagement aus individueller Perspektive

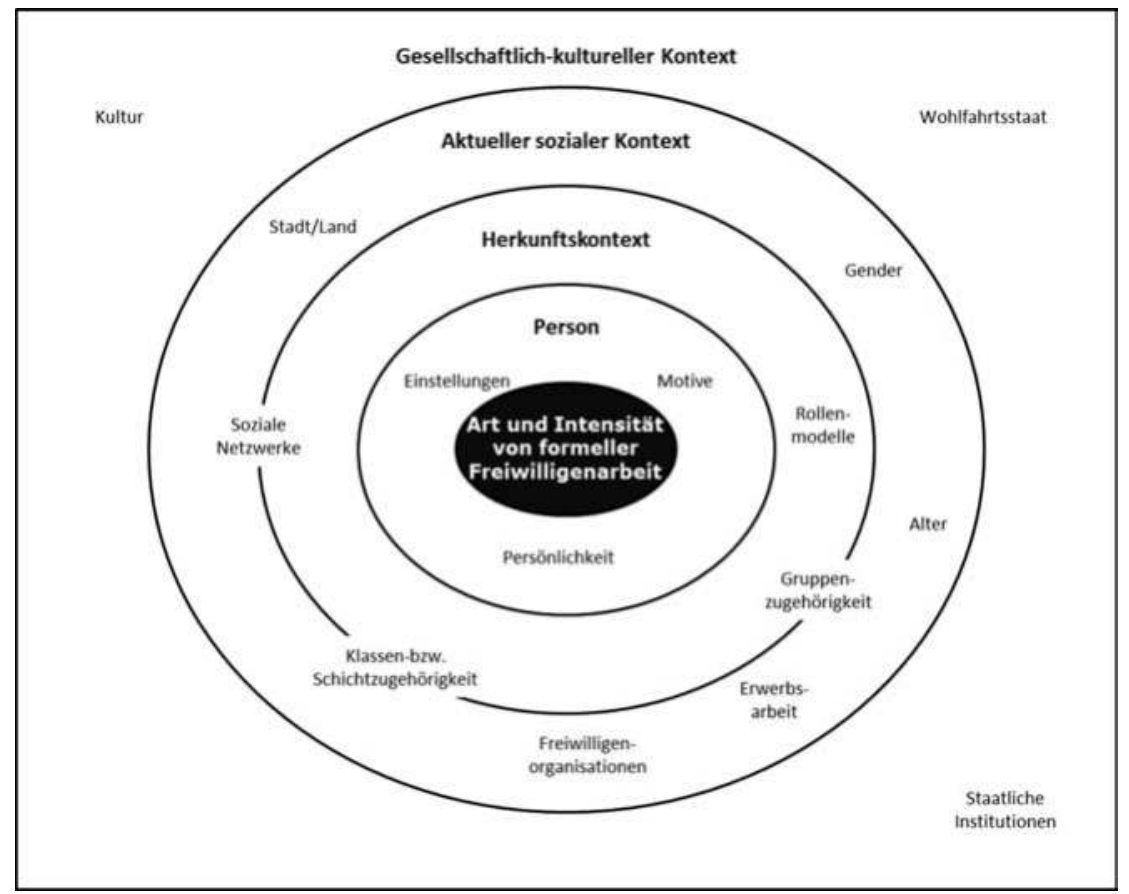

Quelle: Meyer/Rameder $(2011)^{38}$.

Der Fokus der vorliegenden Arbeit ist dabei primär auf den Einfluss der askriptiven (Geschlecht, Alter, ethnische Herkunft) und erworbenen (Bildungsgrad, Berufsstatus, etc.) sozialen Merkmale gerichtet. Wie das Kreismodell zu den Einflussfaktoren in Abbildung 12 zeigt, können diese Merkmale auf der Ebene des aktuellen sozialen Kontextes, d.h. u.a. der aktuellen sozialen Bewertung von Gender, Alter, Erwerbs- und Berufsstatus, sowie auf der vom Herkunftskontext abhängigen Ebene der Ausstattung an kulturellem (Bildungsgrad), sozialem (soziale Netzwerke) und ökonomischen (Vermögen) Kapitalien verortet werden. Bevor der Stand der Forschung zu den askriptiven wie erworbenen Merkmalen als Determinanten der Freiwilligenarbeit (Kap. (II).3.) im Detail beschrieben wird, werden die zentralen Erkenntnisse zu den Einflussfaktoren auf den

38 Die Grafik basiert auf den Arbeiten von Mayrhofer et al. (2007). 
Ebenen des gesellschaftlich-kulturellen Kontextes sowie auf Ebene der Persönlichkeit präsentiert.

Auf gesellschaftlich-kultureller Ebene sind vordergründig der Nonprofitsektor (z.B. die Vereinslandschaft), die staatlichen Institutionen, die etwa die rechtlichen Rahmenbedingungen festlegen sowie die Ausgestaltung des Wohlfahrtsstaates prägende Einflussfaktoren (vgl. u.a. DiMaggio/Anheier 1990; Hustinx et al. 2010b; Matsunaga et al. 2010; Salamon/Anheier 1998; Stadelmann-Steffen 2011). Studien dazu beschäftigt sich u.a. mit den nationalen Unterschieden in der Beteiligungsintensität und -struktur (vgl. Dekker/van den Broek 2005; Plagnol/Huppert 2010; Rotolo/Wilson 2012) sowie dem Einfluss gesamtgesellschaftlicher Heterogenitäts- und Ungleichheitsdimensionen auf die Ausprägung des Nonprofit-Sektors (vgl. Stater 2010). In Europa zeigen sich diesbezüglich große Unterschiede bei den Beteiligungsquoten ${ }^{39}$. Die skandinavischen Staaten weisen gemeinsam mit den Niederlanden die höchsten Beteiligungsquoten auf, während in Süd- und Osteuropa sich prozentuell die wenigsten Menschen freiwillig engagieren (vgl. Mackerle-Bixa et al. 2009). Diese Unterschiede sind sowohl auf politische wie kulturelle Faktoren (vgl. Plagnol/Huppert 2010; van Deth et al. 2007) als auch auf die unterschiedliche Ausprägung des Wohlfahrtsstaats (vgl. Esping-Andersen 1990; Salamon/Sokolowski 2001) zurückzuführen und stehen vielfach in Zusammenhang mit der länderspezifischen Bedeutung von Kirche und Familie (vgl. Parboteeah et al. 2004). Mit dem Einfluss unterschiedlicher Ausgestaltungsformen demokratischer Regierungsformen haben sich Stadelmann-Steffen/Freitag (2011) am Beispiel der Schweizer Kantonalregierungen beschäftigt. Höhere Engagementquoten werden in jenen Kantonen beobachtet, die entweder eine primär repräsentative, oder aber direkte Form der Demokratie anwenden. Jene Kantonen, in denen die beiden Demokratiemodelle und die damit teilweise widersprüchlichen Logiken kombiniert werden, weisen geringere Engagement- und Partizipationsquoten auf.

"By delegating powers either toward [sic] or away from the individual, both logics generate an environment that promotes voluntary engagement. On the other hand, a mixture of a representative conception of democracy with a relatively substantial access to direct democratic instruments may be disadvantageous in terms of civic engagement because the two modes of democracy follow very different mechanisms" (Stadelmann-Steffen/ Freitag 2011: 542f).

39 Die Beteiligungsquote beschreibt den Anteil der Freiwilligen an der jeweiligen Grundgesamtheit in der Bevölkerung. Zu Abgrenzung der Begriffe „Beteiligungsquote“, „Beteiligungsstruktur" und Beteiligungsintensität siehe Rameder/More-Hollerweger (2009: 50). 
Vor dem Hintergrund der aktuellen Finanzkrise ist auch die Frage nach dem Einfluss von Einkommensungleichheit (z.B. Gini-Koeffizient) auf die Freiwilligenarbeit bedeutsam (vgl. Hackl et al. 2007, 2012; Lancee/Van de Werfhorst 2012). Park/Subramanian (2012) kommen diesbezüglich zu dem Ergebnis, dass die positiven Effekte der Mitgliedschaft in freiwilligen Vereinigungen mit dem Grad der Einkommensungleichheit abnehmen. Stater (2010) wiederum beschäftigt sich mit den Einflussfaktoren auf die Heterogenität des Nonprofitsektors und dabei u.a. mit der Frage, in welchem Ausmaß die unterschiedlichen Interessen und Bedürfnisse der Bevölkerung in der Heterogenität des Nonprofit Sektors Wiederhall finden. Ausgehend von den beachtlichen Unterschieden in den Beteiligungsquoten zwischen den einzelnen US Bundestaaten (zwischen 18\% und 44\%) analysieren Rotolo/Wilson (2012) den Einfluss demografischer, institutioneller und kultureller Faktoren auf die Freiwilligenarbeit. Die Ergebnisse zeigen, dass die Höhe des Bildungsgrads nur auf individueller Ebene und nicht auf Makroebene die Beteiligung an der Freiwilligenarbeit positiv beeinflusst: „[H]aving a higher proportion of residents with college degrees or more is not associated with higher levels volunteering" (Rotolo/Wilson 2012: 466). Die Autoren finden dabei sogar einen negativen Effekt eines höheren Bildungsstands der Bevölkerung für religiöse Freiwilligenarbeit, nicht jedoch für säkulares Engagement. Rotolo/Wilson (2012) erklären sich diesen Unterschied wie folgt: „Education might well be an individual resource as far as volunteering is concerned but living in an environment of highly educated people seems to be a disincentive to religious volunteering" (467). Die Erwerbsquote in einer Region hat interessanterweise keinen Einfluss auf die Engagementquote. Die regionale Dichte an Nonprofit Organisationen steht wiederum nur mit nicht-religiösen Formen der Freiwilligenarbeit in positivem Zusammenhang (ebd.).

In Österreich und Deutschland sinken mit der Wohnortgröße und dem Urbanisierungsgrad die Engagementquoten signifikant, wohingegen sich dieser Zusammenhang in der Schweiz nicht zeigt (vgl. Meyer/Rameder 2011). Der geringere Anteil an Freiwilligen in urbanen Zentren wird dabei unterschiedlich erklärt. Einerseits spielen die höhere Mobilität und die heterogenere $\mathrm{Zu}$ sammensetzung der Bevölkerung in Städten eine Rolle, andererseits werden mit steigender Bevölkerungsdichte auch mehr Leistungen von öffentlicher bzw. auch privatwirtschaftlicher Seite abgedeckt (z.B. durch Berufsfeuerwehren, kommerzielle Sportangebote, ein breiteres Angebot an Kulturgütern - Museen, Theater etc.) sodass Freiwilligenarbeit weniger notwendig ist als in ländlicheren Regionen. Nicht zuletzt scheinen die soziale Solidarität und Kohäsion wie auch die Wirksamkeit sozialer Normen in ruralen Gebieten das Freiwillige Engagement zu fördern (vgl. ebd.). Hooghe/Botterman (2012) stellen den negativen Einfluss 
der Urbanisierung auf Freiwilliges Engagement in Frage. Bezogen auf Belgien konnten sie in ihrer Multilevel-Analyse keinen Einfluss des Urbanisierungsgrads auf die Freiwilligenarbeit im gesamten feststellen. Lediglich für das Engagement in traditionellen Freiwilligenorganisationen (Familien- und Seniorenvereinigungen) ist ein negativer Effekt nachweisbar (ebd.).

Betreffend der Persönlichkeitsmerkmale, Einstellungen und Motive belegen Studien, dass vor allem Religiosität positiv mit Freiwilligem Engagement korreliert (vgl. Borgonovi 2008; Taniguchi/Thomas 2011; van Tienen et al. 2011). Positive Einstellungen und die Zufriedenheit mit dem eigenen Leben und der eigenen Gesundheit finden sich häufiger bei Freiwilligen als bei nicht freiwillig engagierten Personen (vgl. Haski-Leventhal 2009; Lum/Lightfoot 2005). Das Vertrauen in andere Menschen ( generalized trust) ist bei Freiwilligen stärker ausgeprägt (vgl. Bekkers 2011; Jung/Kwon 2011; Park/Subramanian 2012; Uslaner/ Brown 2008) und sie sind in geringerem Ausmaß von sozialen Angststörungen $\left(\right.$ social anxiety ${ }^{40}$ ) betroffen (vgl. Handy/Cnaan 2007). Freiwillige sind vielfach extrovertierter und zeichnen sich durch eine vergleichsweise höhere Soziabilität aus (vgl. Elshaug/Metzer 2001). Ebenfalls von Bedeutung ist die Übereinstimmung des eigenen Selbstkonzepts mit jenem der Organisationen (vgl. Randle/ Dolnicar 2011). Der Motiv- und Wertewandel spiegelt sich auch in der Freiwilligenarbeit wieder. Bei den in den großen Surveys üblichen, vorgegebenen Motivabfragen steht der eigenorientierte „Spaß an der Tätigkeit“ jeweils gemeinsam mit dem altruistischen Wunsch „anderen zu helfen“ und dem Bedürfnis nach Sozialkontakten, d.h. „Menschen treffen“, „Freunde gewinnen“ und „gemeinsam etwas bewegen" an vorderster Stelle (vgl. BMASK 2009, 2013; Gensicke/Geiss 2010; Stadelmann-Steffen et al. 2010).

Schlussendlich entscheiden in den meisten Bereichen der formellen Freiwilligenarbeit jedoch die Organisationen, bewusst wie unbewusst, anhand impliziter oder expliziter Kriterien, welche Freiwilligen sie für welche Funktionen aufnehmen und halten (vgl. Meyer/Rameder 2011). Den aktuellen Stand der Forschung zu den organisationalen Einflussfaktoren der Koordination und des Managements von Freiwilligen haben Studer/Schnurbein (2013) in einer umfassenden Literaturanalyse zusammengefasst. Sie diskutieren dabei auch mögliche negative Effekte der Freiwilligenarbeit wie Burnout und Überidentifikation. Die Rolle der Organisationen bei der Genese und Reproduktion sozialer Schließung wird jedoch nicht thematisiert (vgl. Studer/von Schnurbein 2012: 428f). Soziale Gesetzmäßigkeiten wie jene der Homophilie (vgl. McPherson et al. 2001) sind

40 gemessen anhand der Liebowitz Social Anxiety Scale (LSAS). 
möglicherweise gerade bei der Freiwilligenarbeit in geringerem Ausmaß sozial sanktioniert und kommen damit stärker zum Tragen, als dies bei der Erwerbsarbeit der Fall ist. Vor diesem Hintergrund ist die Frage nach Kriterien des Zugangs zu Leitungspositionen, d.h. der Hierarchisierung in der Freiwilligenarbeit, von besonderem Interesse.

\section{(II) 3. Soziale Merkmale als Determinanten des Zugangs zur Freiwilligenarbeit}

Soziale, ökonomische und kulturelle Determinanten der Freiwilligenarbeit beschäftigen die soziologisch-orientierte Freiwilligenforschung in den USA seit den 1970er Jahren (vgl. Lemon et al. 1972; Smith 1975, 1994). In Europa finden sich vor allem seit den 1980er Jahren vermehrt Forschungsarbeiten dazu (z.B. Badelt 1985). Der ungleiche Zugang bezüglich sozialer, ökonomischer und kultureller Merkmale findet sich in nahezu allen empirischen Erhebungen und Studien zur Freiwilligenarbeit bestätigt (vgl. Borgonovi 2008; García-Mainar/ Marcuello 2007; Havens et al. 2006; Huth 2007; Musick/Wilson 2008; Rochester et al. 2010: 191f; Rotolo et al. 2010; Tschirhart 2006; Wilson/Musick 1997a, b, 2000) und kann verdichtet als „Mittelschichtbias der Freiwilligenarbeit“ bezeichnet werden (vgl. Alscher et al. 2009a; Beher 2008; Dathe 2011; Musick/Wilson 2008; Rotolo et al. 2010). Wie bereits in Kapitel (I) ausführlich erläutert sind im Zusammenhang mit sozialer Ungleichheit vordergründig askriptive, d.h. zugeschriebene Merkmale wie Geschlecht, Alter, soziale, regionale und ethnische Herkunft sowie erworbene Merkmale wie Bildungsstand, Berufsprestige, Vermögen und Einkommen von zentraler Bedeutung für die Reproduktion ungleicher Lebensverhältnisse.

Zur Hierarchisierung, d.h. zum Zugang zu den leitenden Positionen und Funktionen innerhalb der Freiwilligenarbeit gibt es bislang vergleichsweise wenige Untersuchungen (vgl. Musick/Wilson 2008; Rotolo/Wilson 2007). Ein Grund dafür ist, dass eine differenzierte Erfassung der Freiwilligenarbeit nach Tätigkeiten bzw. Funktionen in den länderübergreifenden Surveys (EVS, ESS) bisher nicht stattgefunden hat. In den nationalen Erhebungen gibt es zwar oftmals eine detaillierte Erfassung, jedoch ohne einheitliche Operationalisierung. So wird im Rahmen des Freiwilligenmonitors in der Schweiz zwischen (1) allgemeiner Freiwilligenarbeit und (2) Ehrenamt, d.h. gewählten Funktionen unterschieden (Stadelmann-Steffen et al. 2007; Stadelmann-Steffen et al. 2010). Im Rahmen der österreichweiten Erhebungen im Jahr 2006 (Statistik Austria) und 2012 (IFES) wurde hingegen zwischen (1) ausführenden, die Kernaufgaben der Organisation umfassenden Tätigkeiten, (2) administrativen 
und unterstützenden Tätigkeiten, sowie (3) Tätigkeiten, die Leitungsaufgaben umfassen, unterschieden (vgl. BMASK 2009, 2013). Da es, wie bereits Eingangs erwähnt, in den USA im Bereich der Freiwilligenarbeit eine längere Forschungstradition gibt, beziehen sich die meisten Analysen und Ergebnisse auf den USamerikanischen Raum. Musick/Wilson (2008) kommen bei ihrer Analyse der bisherigen Literatur sowie Daten des U.S. Independent Sector survey (1992, 1994, und 1996) zu dem Ergebnis, dass ethnische Herkunft, der Beschäftigungsstatus (full-time, part-time, retired) sowie der Kirchenbesuch die hierarchischen Position in der Freiwilligenarbeit zentral beeinflussen. Gesamt betrachtet zeigt sich, dass in der Freiwilligenarbeit Führungs- und Leitungspositionen eher von Männern (vorwiegend Fach- und Führungskräfte) sowie besser (aus-)gebildeten Personen eingenommen werden. Die Besetzung von Führungspositionen folgt damit in vielen Bereichen der Freiwilligenarbeit den impliziten und expliziten Regeln der Positionsbesetzung in der Erwerbsarbeit (vgl. Rotolo/Wilson 2007; Webb/Abzug 2008). In Europa finden sich zum Thema der internen hierarchischen Strukturierung vor allem Analysen zum Ehrenamt im Feld der Sportvereine (Baur et al. 2003; Hartmann-Tews/Combrink 2006; Nagel 2003a; Radtke 2007; Winkler 1995). Ergänzend finden sich Einzelfallstudien wie sie Beher (2008) zu den „Die vergessene Elite - Führungskräfte in gemeinnützigen Organisationen"veröffentlicht hat.

\section{(II) 3.1. Zugeschriebene Merkmale als Determinanten des Zugangs zur Freiwilligenarbeit}

\section{(II) 3.1.1. Geschlecht}

Das Geschlecht spielt nicht nur in der Erwerbsarbeit sondern auch in der formellen Freiwilligenarbeit auf unterschiedlichen Ebenen eine zentrale Rolle. (1) auf der Ebene des Zugangs zu Freiwilligenarbeit im Allgemeinen, (2) beim Zugang zu spezifischen Engagementfelder und (3) beim Zugang zu bestimmten Tätigkeiten bzw. (hierarchischen) Positionen innerhalb der Freiwilligenorganisationen. Ein Überblick zu aktuellen Studien dazu findet sich bei Musick/Wilson (2008) sowie Wilson (2012). Im Hinblick auf die Beteiligung in Österreich ${ }^{41}$ sowie den meisten anderen europäischen Ländern zeigen die empirischen Daten, dass mehr Männer als Frauen Freiwilligenarbeit leisten

41 Beteiligungsstruktur: 57\% Männer und 43\% Frauen. Dies entspricht einer Beteiligungsquote von 33\% bei den Männern und 23\% bei den Frauen (Statistik Austria 2008). 
(vgl. Gensicke/Geiss 2010; Neumayr/More-Hollerweger 2009; StadelmannSteffen et al. 2010). In Nordamerika hingegen sind mehr Frauen als Männer freiwillig engagiert (vgl. Musick/Wilson 2008; Themudo 2009) und sie leisten durchschnittlich mehr Stunden als Männer (vgl. Taniguchi 2006). Dieser Unterschied zwischen Nordamerika und Europa ist unter anderem darauf zurückzuführen, dass Freiwilligenarbeit in religiösen Kontexten in den USA weitaus verbreiteter ist als in Europa und dieser Bereich traditionell mehr Frauen attrahiert (vgl. Bourdieu 1998: 192; 2005a: 170). Das Geschlecht beeinflusst vor allem in Verbindung mit anderen askriptiven wie erworbenen Merkmalen, der sogenannten Intersektionalität (vgl. Winker/Degele 2009), das Ausmaß und die Form der Freiwilligenarbeit. Dies betrifft vor allem die Interaktion von Geschlecht und Alter (vgl. Fortuijn/van der Meer 2006), Geschlecht und Erwerbsstatus bzw. Berufsstatus (vgl. Marshall/Taniguchi 2012; Popielarz 1999; Tiehen 2000), Geschlecht und Bildungsgrad (vgl. Egerton/Mullan 2008) sowie Geschlecht und Familiensituation hinsichtlich Kinder und Familienstand (vgl. Musick/Wilson 2008; Taniguchi 2006). Den Einfluss, den Kinder auf die Freiwilligenarbeit ihrer Eltern haben ist je nach Alter der Kinder bei Müttern und Vätern höchst unterschiedlich ausgeprägt. „[T]he negative effect of having small children in the house is stronger for mothers than fathers" (Musick/ Wilson 2008: 245). Bei Kindern im Schulalter kehrt sich der Effekt allerdings um. Schulkinder fördern die Freiwilligenarbeit ihrer Mütter mehr als die ihrer Väter (vgl. ebd.: 246). In Österreich findet sich der genderabhängige Einfluss von Kindern teilweise bestätigt (vgl. Neumayr/More-Hollerweger 2009: 101). Frauen mit Kindern unter 3 Jahren im Haushalt weisen eine geringere Beteiligungsquote (17,6\%) auf als Männer (31,2\%) (siehe Tabelle 1). Bei älteren Kindern im Haushalt steigt zwar auch die Beteiligungsquote von Frauen auf 32,1\% an, liegt jedoch weiterhin unter jener, der ebenfalls ansteigenden Beteiligungsquote von Männern mit 38,5\%.

Tabelle 1: Beteiligungsquoten von Frauen und Männern ohne bzw. mit Kindern im Haushalt

\begin{tabular}{lccc}
\hline & \multicolumn{3}{c}{ Beteiligungsquote (\%) } \\
\cline { 2 - 4 } & Frauen & Männer & Gesamt \\
\hline Keine Kinder unter 15 Jahren im Haushalt & 22,5 & 32,4 & 27,5 \\
Kinder unter 3 Jahren im Haushalt & 17,6 & 31,2 & 24,6 \\
Kinder unter 15 Jahren, aber keine Kleinkinder unter 3 & 32,1 & 38,5 & 35,2 \\
Jahren im Haushalt & & & \\
\hline
\end{tabular}

Mikrozensus-Zusatzerhebung (2006); Formelle Freiwilligenarbeit; Basis: Freiwillige; gewichtet (in Anlehnung an Neumayr/More-Hollerweger 2009: 101). 
Neben der Familiensituation wirkt bei Männern und Frauen auch die Erwerbstätigkeit unterschiedlich auf deren Freiwilliges Engagement. Die Art der Erwerbstätigkeit (Job-Charakteristik), das Stundenausmaß, der Status des Berufs sowie eine etwaige Arbeitslosigkeit haben geschlechterspezifische Effekte auf die Freiwilligenarbeit (vgl. Lewis/Noguchi 2006; Taniguchi 2006). „Relative to fulltime employment, part-time employment encourages women's volunteer work but not men's, while unemployment exclusively inhibits men's volunteering" (Taniguchi 2006: 83). Für Österreich kann auf Basis der bestehenden Analysen der oben beschriebene Einfluss (vgl. Taniguchi 2006: 95; Stadelmann-Steffen et al. 2007: 63) nicht gleichermaßen beobachtet werden (vgl. Neumayr/MoreHollerweger 2009: 100). Männer in Teilzeitbeschäftigung weisen hier eine höhere Beteiligungsquote $(51,3 \%$ ) auf als Frauen in Teilzeit (33,0\%) (siehe Tabelle 2).

Tabelle 2: Beteiligungsquoten von Frauen und Männern nach Beschäftigungsausmaß

\begin{tabular}{lccc} 
& \multicolumn{3}{c}{ Beteiligungsquote (\%) } \\
\cline { 2 - 4 } & Frauen & Männer & Gesamt \\
\hline Vollzeit & 24,0 & 35,8 & 31,8 \\
Teilzeit bis 35 Stunden & 33,0 & 51,3 & 35,9 \\
\hline
\end{tabular}

Mikrozensus-Zusatzerhebung (2006); Formelle Freiwilligenarbeit; Basis: Freiwillige; gewichtet (in Anlehnung an Neumayr/More-Hollerweger 2009: 100).

Neben der Art der Beschäftigung (Vollzeit und Teilzeit) hat auch die Art der Tätigkeit (z.B. Führungs- und Leitungsfunktionen) für Frauen und Männer eine unterschiedliche Wirkung. Frauen in leitenden Positionen im Beruf bringen deutlich mehr Zeit für die Freiwilligenarbeit auf als Frauen ohne Leitungsfunktion (vgl. Marshall/Taniguchi 2012):

„Although women are less likely than men to supervise others, being a supervisor promotes women's volunteering but not men's. This finding supports the argument that gender-based expectations extend to the workplace and influence the ways in which job authority is exercised. Since women are expected to be communal, women who exercise supervisory authority may volunteer more than women who do not, while job authority does not seem to matter as much for men's volunteering decisions. Volunteering may be a way for women supervisors to receive positive evaluations since it allows them to balance masculine agentic traits with feminine communal traits, and appear less threatening and more acceptable" (Marshall/Taniguchi 2012: 228).

Der Einfluss der hierarchische Position im Beruf auf die Freiwilligenarbeit ist demzufolge bei Männern und Frauen unterschiedlich ausgeprägt. Der Beruf selbst, dürfte vor allem das Freiwillige Engagement von Männern beeinflussen: 
„Men in manual occupations volunteer significantly less time, either compared to men in managerial/professional occupations or to men in technical/service/clerical/service occupations" (Marshall/Taniguchi 2012: 224).

Rotolo und Wilson (2007) untersuchen anhand amerikanischer Surveydaten $(\mathrm{N}=91.807)$, ob und in welchem Ausmaß es analog zur Erwerbsarbeit zu einer vertikalen und horizontalen Geschlechtersegregation in der Freiwilligenarbeit kommt. Betreffend des Zusammenhangs von Erwerbsarbeit und Freiwilligenarbeit (vgl. dazu auch Staines 1980) formulieren sie zwei Hypothesen. Die Contrast Hypothesis besagt, dass es in der Freiwilligenarbeit zu einer Umkehrung bzw. zumindest Negierung der Geschlechtersegregation anderer Sphären, wie der Erwerbsarbeit oder der Familienarbeit kommt:

„Volunteering is what we do in our 'free time' where, presumably, we have a free choice.

[...] volunteer work offers itself as an alternative stratification system in which the powerless can gain authority and exercise power" (Rotolo/Wilson 2007: 559f).

Demgegenüber steht die Spillover Hypothesis die davon ausgeht, dass die Geschlechtersegregation in der Freiwilligenarbeit jene anderer Arbeitskontexte spiegelt bzw. reproduziert (vgl. Rotolo/Wilson 2007: 562). Als ein Argument für diese Hypothese führen Rotolo/Wilson (2007) an, dass Nonprofit Organisationen und Freiwilligenvereinigungen analog zu gewinnorientierten Unternehmen an einer effizienten Ressourcennutzung interessiert sind und daher bei Freiwilligen ähnliche Recruting-, Trainings- und Managementinstrumente wie bei bezahlten Mitarbeitern einsetzen. Die empirischen Ergebnisse stützen primär die SpilloverHypothese. Rotolo/Wilson (2007) erklären diesen Befund damit, dass Freiwilligenarbeit oftmals in ähnlicher Art und Weise organisiert ist wie die Erwerbsarbeit sowie die Haus- und Familienarbeit und die Aufgaben und Positionen in der Freiwilligenarbeit vielfach ebenso hierarchisch strukturiert sind. Ihre eigenen multivariaten Analysen stützen ebenfalls die Spillover-Hypothese. Männer finden sich traditioneller Weise häufiger als Frauen in „Boards“ und „Committees“ von Freiwilligenorganisationen. Rotolo/Wilson (2007) ergänzen jedoch, dass unter Kontrolle der Klassenzugehörigkeit die Wahrscheinlichkeit in Vorstandspositionen oder Komitees zu kommen bei Frauen uns Männer annähernd gleich ist:

„Gender has a strong influence on horizontal sex segregation, helping sort people into sex-linked jobs at more or less the same status level, but a more modest influence on the vertical dimension [...] The vertical division of labor seems to be influenced more by social class than gender" (580).

In Österreich (Tabelle 3) zeigen deskriptive Analysen bezüglich der horizontalen Geschlechtersegregation, dass mit 82 \% der Männeranteil im Bereich der Katastrophenhilfe am höchsten ist, gefolgt vom Feld des Sports (72\%) und der Politik 
(72\%) (Neumayr/More-Hollerweger 2009: 97). Der Frauenanteil ist wiederum im Feld der Religion (69\%) und der Bildung (65\%) am größten. Ähnliche Ergebnisse finden sich auch in Deutschland (Gensicke/Geiss 2010) und der Schweiz (Stadelmann-Steffen et al. 2010).

Tabelle 3: Beteiligungsstruktur nach Geschlecht in den Bereichen der formellen Freiwilligenarbeit

\begin{tabular}{lccccc}
\hline & \multicolumn{2}{c}{ In diesem } & Bereich tätig (\%) & & \multicolumn{2}{c}{ In diesem Bereich leitend tätig (\%) } \\
\cline { 2 - 3 } \cline { 5 - 6 } & Frauen & Männer & & Frauen & Männer \\
\hline Katastrophenhilfe & 18 & 82 & 9 & 91 \\
Politik & 28 & 72 & & 22 & 78 \\
Sport & 28 & 72 & & 17 & 83 \\
Umwelt & 35 & 65 & & 14 & 86 \\
Gemeinwesen & 37 & 63 & & 28 & 72 \\
Kultur & 46 & 54 & & 29 & 71 \\
Soziales & 53 & 47 & & 46 & 54 \\
Bildung & 65 & 35 & 52 & 48 \\
Religion & 69 & 31 & 59 & 41 \\
\hline Gesamt & $\mathbf{4 3}$ & $\mathbf{5 7}$ & $\mathbf{2 9}$ & $\mathbf{7 1}$ \\
\hline
\end{tabular}

Mikrozensus-Zusatzerhebung (2006); Formelle Freiwilligenarbeit; Basis: Freiwillige; gewichtet (in Anlehnung an: Neumayr/More-Hollerweger 2009: 98).

Betreffend der vertikalen Geschlechtersegregation sind bis auf die Engagementfelder der Bildung und der Religion Männer überproportional in leitenden Positionen vertreten (siehe Tabelle 3). Auch in der Schweiz haben deutlich mehr Männer als Frauen ein gewähltes Ehrenamt inne (vgl. Stadelmann-Steffen et al. 2010) und für Deutschland lässt sich resümieren, dass leitende Positionen häufiger mit gut ausgebildeten, älteren Männern besetzt werden (vgl. Gensicke/Geiss 2010). Erklärungsansätze beziehen sich ebenso auf persistente Geschlechterstereotypen hinsichtlich der tradierten Arbeitsteilung zwischen den Geschlechtern (vgl. Eagly 2009) sowie auf bereits genannten Spillover-Effekt der Erwerbsarbeit auf die Freiwilligenarbeit (vgl. Marshall/Taniguchi 2012; Wilson/Musick 1997b). Gesamt betrachtet sind es jedoch nur eine Handvoll Studien die sich explizit mit der geschlechtsspezifischen, horizontalen wie vertikalen Segregation innerhalb der Freiwilligenarbeit beschäftigen (vgl. Messner/Bozada-Deas 2009; Popielarz 1999; Rotolo/Wilson 2007). Zu einem der Kernthemen der vorliegenden Arbeit, der Frage nach den „gläsernen Wänden“ zwischen den 
Engagementfeldern und den "gläsernen Decken“ zwischen den hierarchischen Positionen der Freiwilligenorganisationen (vgl. Guy/Newman 2004) gibt es noch weiteren Forschungsbedarf. Bei der Analyse der Geschlechterrollen und stereotypen Reproduktionsmuster gilt es die generelle Machtasymmetrien zwischen der Erwerbsarbeit und der Freiwilligenarbeit mitzudenken (vgl. Baines/Hardill 2008; Little 1997). „Within both traditions [philanthropy and mutual aid] there is a longstanding association of volunteering with women's unpaid roles in social reproduction and reciprocal exchange“ (Baines/Hardill 2008: 308).

\section{(II) 3.1.2. Alter}

Das Alter ist neben dem Geschlecht ein weiterer zentraler Einflussfaktor auf Freiwilliges Engagement (vgl. Aner 2007; Aner/Hammerschmidt 2008; Backes/Höltge 2008; Gensicke 2008; Meyer et al. 2009; Musick/Wilson 2008; Schroeter 2006; Suanet et al. 2009; Tang 2006). Im Fokus steht dabei einerseits das Freiwillige Engagement von Jugendlichen und dessen Auswirkungen auf deren Persönlichkeit, deren gesellschaftliche Partizipation im Allgemeinen sowie deren spätere Karriere im Beruf (vgl. Handy et al. 2010; Janoski/Wilson 1995; Oesterle et al. 2004; Sundeen/Raskoff 2000). Andererseits stellt sich die Frage nach den individuellen wie gesellschaftlichen Voraussetzungen und Auswirkungen von Freiwilligem Engagement älterer Menschen, insbesondere jener im Ruhestand. Ein aktuelles Forschungsfeld legt dabei den Fokus primär auf die Effekte der Freiwilligenarbeit auf die Gesundheit, das Wohlbefinden und die Lebenszufriedenheit älterer Menschen (vgl. Backes 2005; Caro 2008; Erlinghagen 2008, 2010; Hank/Erlinghagen 2008; Morrow-Howell 2010; Mühlpfordt 2006; Mutchler et al. 2003; Placke/ Riess 2006; Syvertsen et al. 2011; Tang 2008; Wouters 2005). Gesamt betrachtet decken sich die meisten empirischen Befunde zum Zusammenhang von Alter und Freiwilligenarbeit. Die Beteiligungsquoten erreichen rund um die Lebensmitte ihren Höchststand und sinken danach langsam, mit dem Pensionsantritt dann deutlich ab (vgl. Gensicke et al. 2006; More-Hollerweger/Rameder 2009; Stadelmann-Steffen et al. 2010). Mit einem Austritt aus der Erwerbsarbeit ist oftmals auch das Ende des Freiwilligen Engagements verbunden. Die Gründe dafür sind vielfältig (vgl. Backes 2005; Meyer et al. 2009). Erlinghagen (2006; 2008) hat nachgewiesen, dass Menschen sich im Ruhestand eher freiwillig engagieren, wenn Freiwilligenarbeit bereits in ihrem bisherigen Lebenslauf ein Handlungsfeld dargestellt hat (Erlinghagen 2008, 2010; Erlinghagen et al. 2006). Diese Erkenntnisse sind im Besonderen im Kontext gesundheitlicher Ungleichheit im Alter von Bedeutung. Freiwilliges Engagement als Ressource für „aktives“ und damit „gesünderes“ Altern ist daher nicht erst zum Zeitpunkt des Bedarfs z.B. 
mit „Senioren- und Freiwilligenmessen“ förderbar sondern bedarf einer strukturellen Perspektive über den gesamten Lebenslauf.

\section{(II) 3.1.3. Ethnische und geographische Herkunft}

Die (ethnische) Herkunft spielt ebenfalls eine bedeutsame Rolle beim Zugang zur Freiwilligenarbeit. Die Freiwilligenforschung zum Themengebiet der Migration hat dabei in Nordamerika eine entsprechend längere Tradition als beispielsweise in Europa (vgl. Handy/Greenspan 2009; Kazemipur 2011; Sundeen et al. 2009; Wilson/Musick 2000). In Österreich steht die Forschung diesbezüglich noch am Beginn (vgl. Reinprecht 2009). Grundlegend lässt sich jedoch auf Basis der bestehenden Daten zusammenfassend sagen, dass die Beteiligungsquoten von Personen mit Migrationshintergrund deutlich unter dem Bevölkerungsdurchschnitt liegen (vgl. Alscher et al. 2009a; Reinprecht 2009; Stadelmann-Steffen et al. 2010). Die Beteiligungsquoten von Personen mit Migrationshintergrund liegen in Österreich mit rund 19\% deutlich unter dem Bevölkerungsdurchschnitt von 28\% (vgl. Reinprecht 2009). Carabain/Bekkers (2011) kommen für die Niederlande zu dem Ergebnis, dass Migrantinnen und Migranten vergleichsweise wenig Freiwilligenarbeit für säkulare Organisationen leisten, sich jedoch umso häufiger in religiösen Organisationen freiwillig betätigen. Generell wird in aktuellen Studien zur ethnischen Herkunft und Freiwilligenarbeit der Religion hohes Erklärungspotential zugeschrieben (vgl. Bekkers/Schuyt 2008; Carabain/ Bekkers 2011; Wilson/Musick 2000). Das Zusammenspiel der ethnischen Herkunft mit anderen sozio-ökonomischen Einflussfaktoren wie z.B. dem oftmals geringeren Bildungsgrad, der fehlenden bzw. schlecht bezahlten Erwerbsarbeit oder aber der, aufgrund eines fehlenden Aufenthaltstitel bedingten, Gettoisierung und Stigmatisierung, ist im Kontext der Freiwilligenarbeit bislang kaum erforscht worden. Forschungsmethodisch besteht weiterhin das Problem, dass das Thema "Migration“ in den bestehenden Erhebungsinstrumenten und damit in den Daten nicht repräsentativ abgebildet ist. Viele nationale Erhebungen werden nur in der Landessprache durchgeführt und schließen damit einen nicht unerheblichen Teil der jeweiligen Migrationspopulation davon aus. Zumindest für Österreich gesprochen sind demzufolge die Daten zum Zugang von Personen mit Migrationshintergrund zur Freiwilligenarbeit wie zu ehrenamtlichen Leitungspositionen wenig aussagekräftig.

\section{(II) 3.1.4. Soziale Herkunft}

Die soziale Herkunft, d.h. der Bildungsgrad, der Berufsstatus und das Einkommen der Eltern bzw. des sozialen Umfelds, ist trotz Bildungsexpansion weiterhin 
einer der zentralen Ausgangspunkte der Reproduktion sozialer Ungleichheit (vgl. Berger et al. 2011; Bourdieu 2000 (1973)). In diesem Zusammenhang stellt sich die Frage, welchen Einfluss die soziale Herkunft auf den Zugang zur Freiwilligenarbeit hat (vgl. Bekkers 2007; Janoski et al. 1998; Janoski/Wilson 1995; Oesterle et al. 2004). Auch der Zusammenhang von sozialer Mobilität und Freiwilligem Engagement ist diesbezüglich von Interessen. Laut Lichter et al. (2002) wirkt sich Armut in der Kindheit negativ auf das spätere prosoziale Verhalten aus, im besonderen Maße auf das von Mädchen. Der Einfluss der sozialen Herkunft, d.h. die Herkunftsfamilie und das erweiterte Herkunftsmilieu, auf Freiwilliges Engagement wird anhand verschiedener soziologischer Theorien erklärt. Diese reichen von allgemeinen Sozialisationstheorien über Role-Modeling-Theorien bis hin zu Status-Transmissions-Theorien, die die Vererbung von Klassen- und Schichtzugehörigkeit erklären (vgl.: Bekkers 2007; Janoski/Wilson 1995; Musick/ Wilson 2008: 225ff). Mustillo et al. (2004) weisen auf Basis von Längsschnittdaten aus den USA der Herkunftsfamilie eine zentrale Funktion im Kontext der Freiwilligenarbeit zu. Sie unterscheiden dabei jedoch zwischen jenen Faktoren, die für die Aufnahme von Freiwilligenarbeit in der Jugend von Bedeutung sind und jenen, die Freiwilligenarbeit im späteren Lebenslauf beeinflussen.

„The effect of family socioeconomic status on initial volunteering operates through mother's volunteering and daughter's education. Later growth in volunteering, however, is completely driven by family socioeconomic status" (Mustillo et al. 2004: 538).

Bekkers (2007) überprüft in seiner empirischen Studie die unterschiedlichen Modi der intergenerationalen Weitergabe von Freiwilligem Engagement. Er teilt diese dazu in direkte und indirekte Wege der Transmission ein. Zur direkten Übertragung zählen Banduras (1977) Social Learning Theory. „[P] arents can enhance their children's prosocial behaviour by modelling prosocial behavior" (Bekkers 2007: 100). Als indirekte Wege der Übertragung führt Bekkers (2007) mit Bezug auf Max Weber die Statusübertragung an: „[T]he transmission of volunteering is a by-product of the transmission of religion, social status and personality characteristics" (111). Durch die Weitergabe eines (hohen) sozio-ökonomischen Status werden Ressourcen übertragen, die für Freiwilliges Engagement förderlich sind (vgl. Bekkers 2007: 101; Wilson 2000). Die empirischen Ergebnisse von Bekkers stützen die indirekte TransmissionsThese bezogen auf Freiwilligenarbeit in religiösen und quasi-religiösen Vereinigung. In diesem Zusammenhang fragen Vermeer/Scheepers (2012) nach der Auswirkung religiöser Sozialisation in der Herkunftsfamilie auf nichtreligiöse Freiwilligenarbeit. Sie kommen zu dem Ergebnis, dass aktive Religiosität einen signifikant positiven Einfluss auf Freiwilliges Engagement hat. 
In nicht-religiösen Kontexten scheint darüber hinaus eine nicht zu strenge religiöse Sozialisation einen positiven Effekt auf das Engagement zu haben (Vermeer/Scheepers 2012). Der Religion wird jedoch nicht nur im Kontext der Sozialisation Bedeutung zugeschrieben (vgl. Bekkers 2007; Caputo 2009; Vermeer/Scheepers 2012), sondern gilt als zentraler eigenständiger Faktor, der positiv auf Freiwilliges Engagement wirkt (vgl. Bekkers/Schuyt 2008; Borgonovi 2008; Djupe/Grant 2001; Loveland et al. 2005; Taniguchi/Thomas 2011; van Tienen et al. 2011; Wilson/Musick 2000).

Von diesen Studien (Bekkers 2007; Caputo 2009; Mustillo et al. 2004; Vermeer/Scheepers 2012) abgesehen, finden sich keine weiteren aktuellen empirischen Befunde zum Zusammenhang von sozialer Herkunft und Freiwilligem Engagement. Die diesbezüglich geringe Forschungsdichte in Europa, mit Ausnahme der Niederlande, mag an der im Vergleich zu Nordamerika zeitlich deutlich verzögerten Implementierung von Fragen zur Freiwilligenarbeit in die nationalen wie europaweiten Erhebungsinstrumente der amtlichen Statistiken liegen. Die Erfassung von Daten zur sozialen Herkunft, gemeinsam mit Daten zur Freiwilligenarbeit der Eltern, ist bis heute weder in den zentralen europaweiten Surveys (wie z. B. $\mathrm{EVS}^{42}, \mathrm{ESS}^{43}$ ) noch in den nationalen Erhebungen (vgl. dazu: Freiwilligen-Monitor, Schweiz; Freiwilligensurvey, Deutschland; Mikrozensuszusatzerhebung zu Freiwilligenarbeit, Österreich) umgesetzt worden.

Dem Überblick zum Einfluss der askriptiven Merkmale auf Freiwilliges Engagement folgt nun der Stand der Forschung zu den erworbenen Merkmalen als Determinanten der Freiwilligenarbeit.

\section{(II) 3.2. Erworbene Merkmale als Determinanten des Zugangs zur Freiwilligenarbeit}

\section{(II) 3.2.1. Formaler Bildungsgrad}

Kulturelles Kapital in institutionalisierter Form von Bildungsabschlüssen (bzw. -titeln) ist in nahezu allen Studien einer der konsistentesten Prädiktoren für Freiwilliges Engagement (vgl. Brand 2010: 418; Mitani 2013; Musick/ Wilson 2008: 119ff; Wilson 2000: 219; 2012: 185f). Die Gründe und Ursachen diesbezüglich sind vielfach diskutiert und reichen von höherem Problembewusstsein, mehr Empathie, kritischerem Denken bis dahin, dass der längere

42 European Value Study (http://www.europeanvaluesstudy.eu).

43 European Social Survey (http://www.europeansocialsurvey.org). 
Verbleib im Bildungssystem mehr freie Zeitressourcen und mehr soziale Kontakte während dieser Lebensphase ermöglicht (ebd., Campbell 2009; Gesthuizen/Scheepers 2012). Darüber hinaus ist die Wahrscheinlichkeit „gefragt zu werden" aufgrund heterogenerer sozialer Netzwerk bei höher gebildeten Personen größer als bei den unteren Bildungsschichten (vgl. Musick/Wilson 2008: 120, 126). Der Einfluss des Bildungsgrades auf die Position innerhalb der Freiwilligenarbeit folgt den klassischen Selektionsmustern der Erwerbsarbeit. Freiwillige die über höhere Bildungsabschlüsse verfügen, werden häufiger gefragt „white collar“ Tätigkeiten und Führungsfunktionen zu übernehmen (vgl. Rotolo/Wilson 2007: 563).

Der Zusammenhang von Bildungsgrad und Freiwilligenarbeit (siehe Abbildung 13) wird laut Musick/Wilson (2008) von folgenden weiteren Moderatorund Mediatorvariablen ${ }^{44}$ beeinflusst. Das Geschlecht, die ethnische Herkunft und das Alter haben einen moderierenden Einfluss auf den Zusammenhang von Bildungsgrad und Freiwilligenarbeit. Die empirischen Befunde betreffend den Einfluss des Geschlechts sind jedoch widersprüchlich. Musick/Wilson (2008) konnten in ihren empirischen Analysen selbst keinen moderierenden Effekt des Geschlechts auf den Zusammenhang von Bildungsgrad und Freiwilligenarbeit feststellen. Den moderierenden Effekt der ethnischen Herkunft betreffend konnten sie hingegen nachweisen, dass der Bildungsgrad nur bei weißen Amerikanerinnen und Amerikanern positiv mit der Anzahl der geleisteten Freiwilligenstunden korreliert, nicht jedoch bei Afroamerikanerinnen und Afroamerikanern. Höheres Bildungskapital wirkt sich darüber hinaus stärker auf die Beteiligung an der Freiwilligenarbeit von Personen mittleren Alters (40 bis 59 Jahren) als von jüngeren Personen aus. Für Musick/Wilson (2008) bestätigt sich damit ihre Hyopothese, ,that education might have a 'sleeper effect' on volunteering" (125).

44 Als Moderatorvariablen bezeichnet man Variablen (z.B. Geschlecht, Alter, etc.), die den Zusammenhang zwischen einer abhängigen Variable (z.B. Freiwilligenarbeit) und einer unabhängigen Variable (z.B. Bildungsgrad) beeinflussen. D.h. Variable „C“ beeinflusst (moderiert) den Zusammenhang zwischen Variable „A“ und „B“. Im Gegensatz dazu handelt es sich bei Mediatorvariablen um sogenannte „Drittvariablen“ (z.B. Größe und Art des sozialen Netzwerks, subjektive Dispositionen) die den Zusammenhang zwischen einer abhängigen Variable (z.B. Freiwilligenarbeit) und einer unabhängigen Variable (z.B. Bildungsgrad) vermitteln (mediieren). D.h Variable „A“ wirkt auf Variable „B“, indem „A“ zuerst auf „C“ wirkt und „C“ wiederum auf „B“ wirkt. 
Abbildung 13: Moderator- und Mediatorvariablen des Zusammenhangs von Bildungsgrad und Freiwilligenarbeit

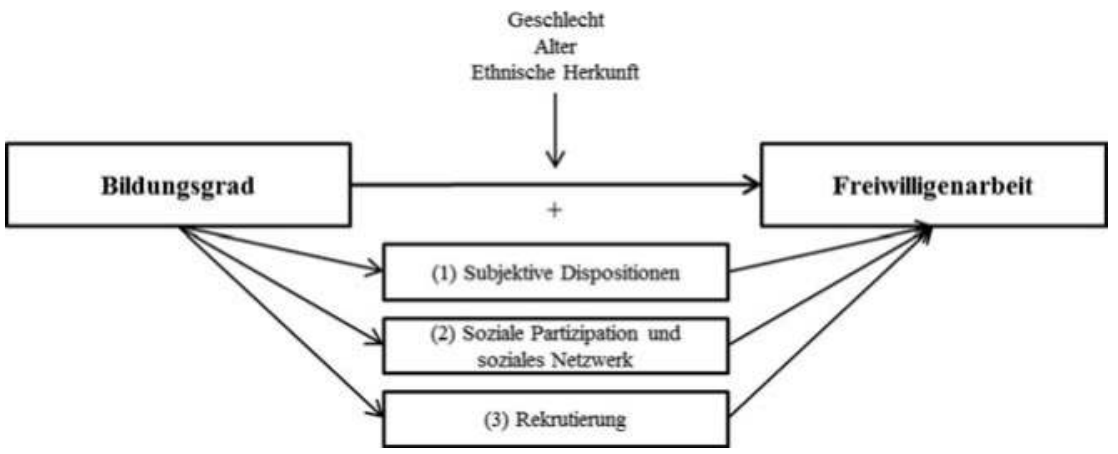

Quelle: Musick/Wilson (2008: 124ff); eigene Darstellung.

Subjektive Dispositionen, die Art und Größe des sozialen Netzwerks und die höhere Wahrscheinlichkeit rekrutiert zu werden haben hingegen keinen moderierenden, sondern einen mediierenden Einfluss (vgl. 124ff). (1) Höhere Bildung ist oftmals mit einer höheren Empathiefähigkeit, einer größeren Selbstwirksamkeitserwartung sowie vermehrtem Vertrauen in andere Menschen bzw. die Gesellschaft verbunden, alles Faktoren, die wiederum selbst positiv mit Freiwilligem Engagement korrelieren. (2) Höher gebildete Personen partizipieren in einem höheren Ausmaß am sozialen Leben. Musick/Wilson (2008) haben dies anhand der Anzahl der Organisationsmitgliedschaften als auch der informellen Kontakte bzw. Interaktionen gemessen. Bei ihren empirischen Analysen erklärt die Anzahl der Organisationsmitgliedschaften und der sozialen Interaktionen mehr als die Hälfte des Effekts des Bildungsgrads auf die Freiwilligenarbeit (ebd.: 126). (3) Personen mit höheren Bildungsabschlüssen haben, wie bereits angeführt, umfangreichere und heterogenere soziale Netzwerke und werden aufgrund der ability signaling Funktion von Bildungstiteln (vgl. Spence 1973) häufiger von anderen Personen für freiwillige Tätigkeiten rekrutiert.

Aus einer weiteren Perspektive stellt sich aufgrund der persistenten Korrelation von Bildungsgrad und sozialer Herkunft die Frage, ob nicht vor diesem Hintergrund der Bildungsgrad primär eine Mediatorvariable des Zusammenhangs von Freiwilligenarbeit und sozialer Herkunft darstellt. Egerton (2002) versucht diese beiden Effekte anhand von Daten von Schülern und Studenten zu entflechten und kommt zu dem Ergebnis, dass der Einfluss von höherer Bildung einen zusätzlichen, wenn auch sehr geringen, Erklärungswert abseits der soziale Herkunftsbedingungen liefert (Egeraton 2002). 
Abseits des Einflusses des individuellen Bildungsgrades gibt es, wie bereits weiter oben mit der Arbeit von Rotolo/Wilson (2012) angesprochen wurde, Forschungsarbeiten, die sich mit dem Makro-Level-Einfluss des Bildungsstands der Bevölkerung auf die Freiwilligenarbeit beschäftigt (vgl. Campbell 2009; Gesthuizen/Scheepers 2012; Rotolo/Wilson 2012) sowie dessen veränderten Einfluss im Zeitverlauf untersuchen (vgl. Syvertsen et al. 2011; van Ingen/Dekker 2010). Auch zwischen den einzelnen europäischen Staaten unterscheidet sich der $\mathrm{Zu}$ sammenhang von Bildungsgrad und Freiwilligenarbeit (vgl. Gesthuizen/Scheepers 2012). Campbell (2009) untersucht in seiner Studie die These, dass Bildung keinen absoluten, sondern einen relativen Einfluss auf zivilgesellschaftliches Engagement hat. Er begründet dies damit, dass der Bildungsgrad auch als Marker für sozialen Status interpretiert werden kann und die Höhe des Status von Ego, vom Status von Alter abhängt. Das sogenannte Sorting Model des Bildungsgrades konnte Campbell (2009) nur für politische Partizipation im Kontext von Wahlen und das primär für Männer bestätigten. Als Conclusio formuliert er folgende Empfehlung:

„Policymakers should be encouraged that the public investment in education appears to have a civic payoff after all. At a time when an instrumental, careerist perspective on education dominates public discourse, we are reminded that education - at all levels has a civic dimension as well“ (Campbell 2009).

Abschließend sei erwähnt, dass nur wenige Studien den Zusammenhang von Bildungsgrad und Freiwilligenarbeit hinterfragen (vgl. Brand 2010; Diekmann 2005; Schnittker/Behrman 2012; van Ingen/Dekker 2010).

Im Zusammenhang mit der Reproduktion sozialer Ungleichheit stellt sich die Frage, ob der Bildungsgrad (verstanden als kulturelles Kapital) nicht nur Voraussetzung, sondern auch durch Freiwilligenarbeit in Form von Zertifikaten und Bestätigungen (von z.B. sozialer Kompetenz) akkumuliert wird. Ein Trend zur Zertifizierung der Freiwilligenarbeit (z.B. österreichischer Freiwilligenpass) ist jedenfalls beobachtbar. Aufgrund fehlender Längsschnittuntersuchungen kann die Frage nach der Reproduktion von Bildungsungleichheiten durch die Freiwilligenarbeit derzeit nicht beantwortet werden.

\section{(II) 3.2.2. Erwerbstätigkeit und berufliche Stellung}

Die Erwerbstätigkeit beeinflusst auf differenzierte Weise die Freiwilligenarbeit. Die Art der beruflichen Tätigkeit sowie das damit verbundene symbolische Kapitel, d.h. der Wert und die Anerkennung, die man in den sozialen Feldern durch die Art der beruflichen Tätigkeit erfährt, prägen auch die Position und Funktion 
in der freiwilligen Tätigkeit. Der Einfluss des Beschäftigungsausmaßes (Teilzeit/ Vollzeit) sowie der beruflichen Stellung wurden bereits im Zusammenhang mit dem Geschlecht diskutiert. Ergänzend dazu werden die empirischen Befunde zur Bedeutung von Arbeitslosigkeit sowie von der beruflichen Stellung für die Position in der Freiwilligenarbeit beschrieben. In nahezu allen Erhebungen zur Freiwilligenarbeit liegen die Beteiligungsquoten von Arbeitslosen (vgl. Erlinghagen 2000; Strauß 2009) sowie generell von erwerbslosen Personen (z.B. haushaltsführende Personen, Personen in Ausbildung, Personen im Ruhestand) deutlich unter dem Bevölkerungsdurchschnitt (vgl. Hustinx 2007; Meyer/Rameder 2011; Musick/Wilson 2008; Webb/Abzug 2008; Wilson/Musick 1997b).

Tabelle 4: Formelle Freiwilligenarbeit - Ausgeübte Funktion und Berufsstatus

\begin{tabular}{lccccc} 
& \multicolumn{2}{c}{ Freiwilligenarbeit } & & \multicolumn{2}{c}{ Funktion (100\%) } \\
\cline { 2 - 3 } \cline { 5 - 6 } & BQ & BS & & leitend & ausführend \\
\hline Nicht-Erwerbstätige & 21,5 & 32,8 & & 18,5 & 81,5 \\
ArbeiterInnen & 27,6 & 15,1 & & 18,0 & 82,0 \\
Mithelfende Familienangehörige & 33,4 & 1,4 & & - \\
Vertragsbedienstete & 46,5 & 4,2 & & 22,1 & 77,9 \\
Angestellte & 31,7 & 29,1 & & 27,4 & 72,6 \\
BeamtInnen & 39,9 & 5,9 & & 39,9 & 60,1 \\
Freie DienstnehmerInnen & 47,3 & 1,4 & & - \\
Selbstständig ohne & 38,2 & 6,0 & & 36,8 & 63,2 \\
ArbeitnehmerInnen & 33,9 & 4,0 & & 41,5 & 58,5 \\
Selbstständig mit & & & & \\
ArbeitnehmerInnen & 27,9 & 100,0 & 24,6 & 75,4 \\
\hline Gesamt & & & &
\end{tabular}

Basis Beteiligungsquoten (BQ): österreichische Wohnbevölkerung; gewichtet.

Basis Beteiligungsstruktur (BS) und Funktion: Freiwillige (formell); gewichtet.

Mikrozensus-Zusatzerhebung (2006); eigene Berechnungen (in Anlehnung an: More-Hollerweger et al. 2009a: 77).

Auf Grund der höchst unterschiedlichen situativen Bedeutung der Arbeitslosigkeit weisen Musick/Wilson (2008: 154) darauf hin, dass der Einfluss der Arbeitslosigkeit, sofern er auf Querschnittdaten basiert, nur im Zusammenhang mit anderen Variablen (Geschlecht, Bildungsgrad, etc.) interpretiert werden sollte. Gesamt betrachtet belegt der Einfluss von beruflichen Tätigkeiten die große Bedeutung der Integration in formale und organisationale Strukturen für den Zugang zu Freiwilligem Engagement. Dies zeigt sich zum Beispiel an der bereits 
erwähnten mediierenden Funktion der Anzahl von Organisationsmitgliedschaften beim Zusammenhang von Bildungsgrad und Freiwilligenarbeit (Musick/ Wilson 2008: 126). Wie in anderen Ländern beeinflusst auch in Österreich die berufliche Stellung die Beteiligungsquote (siehe Tabelle 4). 27,6\% der Arbeiterinnen und Arbeiter engagieren sich freiwillig. Angestellte liegen mit 31,7\% etwas darüber. Deutlich höhere Beteiligungsquoten weisen hingegen Beamtinnen und Beamte (39,9\%), Vertragsbedienstete $(46,5 \%)$ sowie Freie Dienstnehmerinnen und Dienstnehmer (47,3\%) auf.

Auch der positive Zusammenhang zwischen der beruflichen Stellung und der Position (ausführende versus leitende Funktion) in der Freiwilligenarbeit findet sich in Österreich bivariat bestätigt (vgl. More-Hollerweger et al. 2009a). Gemeinsam mit Beamtinnen und Beamten (39,9\%) weisen Selbständige (41,5\%) mit Abstand die höchsten Leitungsquoten ${ }^{45}$ unter den Freiwilligen auf. Somit kann auch in Österreich von einem Spillover-Effekt des Berufs auf die Freiwilligenarbeit ausgegangen werden (vgl. Wilson 2012: 186; Wilson/Musick 1997b). Ehrhardt (2011) beschreibt diesen Aspekt bei gewählten Ehrenämtern in Vereinen wie folgt:

„Da die Vereinsmitglieder die Wahl ihrer Funktionsträger von den erwarteten funktionalen Kompetenzen (nützliche Kontakte, Managementkompetenzen usw.) abhängig machen, die ganz überwiegend aus der Berufstätigkeit der Kandidaten abgeleitet werden, kann man von einer Form der Positionsbesetzung sprechen, bei der die Erwartungen der Kandidaten und die der Mitglieder durch das Wahlverfahren mit einander abgeglichen werden. Je begehrter und wertvoller die Position, desto mehr Ressourcen benötigen Kandidaten, die sie der Organisation anbieten können“ (63).

\section{(II) 3.2.3. Prestige und Status}

Mit dem Beruf geht nicht nur die Entwicklung bestimmter Fähigkeiten und Fertigkeiten einher, sondern dieser ist auch zentrale Bestimmungsgröße für den sozialen Status. Für den Zugang und die interne Positionierung in der Freiwilligenarbeit ist somit nicht nur die Frage, ob man erwerbstätig ist, sondern auch, welchen Beruf man nachgeht, welches soziale Prestige damit verbunden ist und wie hoch das damit verbundene Einkommen ist, von Bedeutung. Bereits Max Weber (1921) hat sich im Zuge seiner "Herrschaftssoziologie“ mit den für ehrenamtliches Engagement in (politischen) Verbänden (sogenannten

45 Anteil an den freiwillig tätigen Beamtinnen und Beamten, die in der Freiwilligenarbeit in einer leitenden Position sind. 
„Honoratioren“) nötigen sozio-ökonomischen Ressourcen sowie den statusbezogenen Konsequenzen beschäftigt:

„[... , Honoratioren' sollen solche Personen heißen, welche

1. kraft ihrer ökonomischen Lage imstande sind, kontinuierlich nebenberuflich in einem Verband leitend und verwaltend ohne Entgelt oder gegen nominalen oder EhrenEntgelt tätig zu sein und welche

2. eine gleichviel worauf beruhende, soziale Schätzung derart genießen, daß sind die Chance haben, bei formaler unmittelbarer Demokratie kraft Vertrauens der Genossen freiwillig, schließlich traditional, die Aemter inne zu haben“ (Weber 1921/1980: 170).

Damit betont Weber sowohl die Bedeutung des ökonomischen als auch des symbolischen Kapitals (Prestige) für derartige ehrenamtliche Positionen. Welche weiteren Voraussetzungen zur damaligen Zeit zentral waren und insbesondere welche Berufe ehrenamtlichen Tätigkeiten ermöglichen, fasst er wie folgt zusammen:

„Unbedingte Voraussetzung der Honoratiorenstellung in dieser primären Bedeutung: für die Politik leben zu können, ohne von ihr leben zu müssen, ist ein spezifischer Grad von ,Abkömmlichkeit' aus eigenen privaten Geschäften. Diesen besitzen in Höchstmaß: Rentner aller Art: Grund-, Sklaven-, Vieh-, Haus-, Wertpapier-Rentner. Demnächst solche Berufstätige, deren Betrieb ihnen die nebenamtliche Erledigung der politischen Geschäfte besonders erleichtert: Saisonbetriebsleiter (daher: Landwirte), Advokaten (weil sie ein ,Bureau' haben) und einzelne Arten anderer freier Berufe. In starkem Maß auch: patrizische Gelegenheitshändler. Im Mindestmaß: gewerbliche Eigenunternehmer und Arbeiter" (Weber 1921/1980: 170).

Trotz der vor allem gegen Ende des 20ten Jahrhunderts zu beobachteten Diversifizierung und Expansion des Ehrenamts und der Freiwilligenarbeit, scheint der Einfluss der genannten sozio-ökonomischen und symbolischen Kapitalien, nicht nur für das politische Engagement, heute noch aktuell zu sein. Jedoch ist Prestige und damit symbolisches Kapital als einer der zentralen Einflussfaktoren in den letzten Jahrzehnten nur sehr selten in den Fokus empirischer Arbeiten gerückt. Dabei gibt es z.B. zahlreiche Hinweise (vgl. auch Tabelle 4), dass in der Freiwilligenarbeit die gesamtgesellschaftlich ungleiche Verteilung von Prestige und damit verbunden sozialer Anerkennung, zum Beispiel zwischen Frauen und Männern, durch die ungleiche Besetzung von ehrenamtlichen und freiwilligen Leitungspositionen noch verstärkt wird (vgl. Neumayr/MoreHollerweger 2009; Stadelmann-Steffen et al. 2010). Die sogenannte statistische Diskriminierung ${ }^{46}$ von Frauen ist erwartungsgemäß nicht auf die Erwerbswelt

46 „Von statistischer Diskriminierung wird [...] allgemein dann gesprochen, wenn Entscheidungen über das einzelne Individuum auf Grundlage von Verhaltensannahmen 
beschränkt, sondern findet sich auch in der hierarchischen Organisation der Freiwilligenarbeit wieder. Zum Beispiel sinkt beim Deutschen Alpenverein (DAV) der Frauenanteil (2011) von rund 40\% unter den Mitliedern auf 13\% bei den 2. Vorsitzenden und auf 6\% bei den 1. Vorsitzenden. Das DAV Präsidium ${ }^{47}$ bestand $2011 \mathrm{zu}$ 100\% aus Männern und auch im aktuellen Organigramm ${ }^{48}$ sind nur vier, der insgesamt 27 Positionen der Geschäftsführung und des Verbandsrats (Präsidium, Bundesausschüsse und Regionalvertreter), mit Frauen besetzt. Auch im Österreichischen Alpenverein (ÖAV) setzt sich das Präsidi$\mathrm{um}^{49} \mathrm{zu} 100 \%$ aus Männern zusammen und nur drei der 25 Bundesausschusssitze werden von Frauen besetzt.

Zum Zusammenhang von Status und Freiwilligenarbeit haben bivariate Analysen (Tabelle 5) zur Freiwilligenarbeit in Österreich gezeigt, dass ein positiver Zusammenhang zwischen der Position in der Freiwilligenarbeit (ausführende Funktion versus leitende Funktion) und dem Berufsstatus (ISEI ${ }^{50}$ ) besteht (vgl. More-Hollerweger et al. 2009a: 79).

bezüglich ganzer sozialer Gruppen getroffen werden“ (vgl. Solga et al. 2009: 20). Zum Beispiel, die Annahme von Arbeitgebern, dass Frauen aufgrund von Haushalts- und Kinderbetreuungspflichten zeitlich weniger flexibel sind als Männer. Dies wird unterstellt, ohne tatsächliche über die Lebenssituation der einzelnen Frau Bescheid zu wissen (vgl. ebd.).

47 Quelle: DAV Panorama (3/2012: 55-58): Gabi Funk: Frauen im Ehrenamt. http://www. alpenverein.de/DAV-Services/Panorama-Magazin/Panorama-Archiv/ - abgerufen am 28.5.2014.

48 DAV Organigramm 2014 - Besetzung. http://www.alpenverein.de/der-dav/dasstruktur-des-dav_aid_10415.html - abgerufen am 28.5.2014.

49 http://www.alpenverein.at/portal/der-verein/ueber-uns/praesidium/index.php - abgerufen am 28.5.2014.

50 Internationale Socio-Economic Index (ISEI). Der ISEI kann einen Wert zwischen 16 und 85 annehmen. Je höher der Wert, desto höher ist auch der sozio-ökonomische Status einer Berufsgruppe (vgl. Schimpl-Neimanns 2004). 
Tabelle 5: Formelle Freiwilligenarbeit - Vergleich des sozio-ökonomischen Status (ISEI) erwerbstätiger Personen

\begin{tabular}{|c|c|c|c|c|}
\hline & $\begin{array}{l}\text { keine } \\
\text { Freiwilligenarbeit }\end{array}$ & $\begin{array}{l}\text { Formelle } \\
\text { Freiwilligenarbeit } \\
\text { - ausführende } \\
\text { Funktion }\end{array}$ & $\begin{array}{l}\text { Formelle } \\
\text { Freiwilligenarbeit } \\
\text { - leitende } \\
\text { Funktion }\end{array}$ & $\begin{array}{l}\text { Formelle } \\
\text { Freiwilligenarbeit } \\
\text { - Gesamt }\end{array}$ \\
\hline Männer & 43,1 & 42,2 & 47,7 & 44,0 \\
\hline Frauen & 41,0 & 41,6 & 47,9 & 42,8 \\
\hline Gesamt & 42,1 & 41,9 & 47,7 & 43,5 \\
\hline
\end{tabular}

Formelle Freiwilligenarbeit; gewichtet.

ISEI (auf Basis ISCOO 883 Steller) Minimalwert: 16; Maximalwert 85.

Mikrozensus-Zusatzerhebung (2006); eigene Berechnungen (in Anlehnung an: More-Hollerweger et al. 2009a: 79).

Gesamt betrachtet weisen berufstätige Personen, die keine Freiwilligenarbeit leisten, mit einem durchschnittlichen ISEI-Wert von 42,1 einen signifikant niedrigeren sozio-ökonomischen Status auf als erwerbstätige Personen, die ehrenamtlich engagiert sind, mit einem ISEI-Wert von 43,5 (MoreHollerweger et al. 2009a: 79). Dieser Unterschied ist jedoch nur bei Frauen $(41,0 \mathrm{zu} 42,8)$ und nicht bei Männern (43,1 zu 44,0) signifikant (ebd.). Die ISEI-Werte von Freiwilligen mit Leitungsfunktion liegen mit 47,7 signifikant über den ISEI-Werten von Freiwilligen, die mit ausführenden Aufgaben betraut sind $(41,9)$ (vgl. More-Hollerweger et al. 2009a: 79). Auffällig ist, dass es bei den leitenden Funktionen in der Freiwilligenarbeit nur einen äußerst geringen und nicht signifikanten Unterschied zwischen Frauen $(47,9)$ und Männern $(47,7)$ gibt. Vor allem beruflich erfolgreiche Frauen scheinen es trotz der gläsernen Decken zu schaffen, in leitende Positionen zu gelangen (vgl. Rotolo/Wilson 2007).

Bei der Freiwilligenarbeit ist, in Abhängigkeit des Engagementbereichs, auch von wechselseitigen (symbolischen) Profiten zwischen den Organisationen und den Individuen auszugehen.

„Just as people exploit their cultural capital to obtain volunteer opportunities, so organizations offer purposive incentives in form of symbolic and expressive 'goods “" (Wilson/ Musick 1997, 709).

Handy/Mook (2011) gehen davon aus, dass es sich bei Freiwilligenarbeit in Gremien oder Vorständen um eine Form des gegenseitigen Prestigekonsums (conspicuous consumtion) handelt, d.h. diese sowohl für die Freiwilligen als auch für die Organisation mit Prestigegewinnen sowie mit einer Ausweitung des sozialen und beruflichen Netzwerks verbunden sind (Handy/Mook 2011: 413). 
„For those institutions symbolic of cultural capital - such as universities, museums, orchestras, large foundations, and nonprofits - status-enhancing prestige is a highly sought after by-product of volunteering. Unlike donations, which anyone can make, board seats are extremely limited, thereby creating an aura of selectivity" (Handy/Mook 2011: 414).

Je elitärer die Organisation, desto mehr Prestige wird mit einer ehrenamtlichen Vorstandsposition verbunden (vgl. Ostrower 2002). Dieser Perspektive folgen auch Ehrhardts (2011) Überlegungen zum Prestigegewinn bei Ehrenämtern, die er unter Bezug auf Webers (1920) Ausführungen zum Ehrenamt anstellt:

„Je höher die Wertschätzung des Amtes, desto mehr erhält der Stelleninhaber qua Reputation für seinen Arbeitsaufwand (Arbeitszeit) zurück. Folglich kann man erwarten: je höher das Prestige des Amtes, desto länger die Amtsdauer. [...] Das Ehrenamt, das dem Amtsinhaber Prestige vermittelt, ist mit dem Leistungsprinzip verknüpft“ (Ehrhardt 2011: 65).

\section{(II) 3.2.4. Einkommen und Vermögen}

Der materielle Wohlstand geht vielfach Hand in Hand mit dem Bildungsgrad und der beruflichen Position. Insofern bestätigen die Studien, dass das Einkommen und Vermögen einen überwiegend positiven Einfluss auf Freiwilliges Engagement hat (vgl. Musick/Wilson 2008: 128; Pearce 1993; Rochester et al. 2010: 45; Tang 2006). Der Einfluss von ökonomischem Kapital auf die Freiwilligenarbeit ist jedoch nicht linear. Mittlere Einkommen wirken sich positiv und vergleichsweise niedrige und vergleichsweise sehr hohe Einkommen wirken sich negativ auf das Engagement aus. Dies bestätigt den schon erwähnten „Mittelschichtbias" des Zugangs zur Freiwilligenarbeit. Einer aktuellen Erhebung des Markt- und Meinungsforschungsinstitutes IFES ${ }^{51}$ aus dem Jahr 2012 zufolge, steigt in Österreich mit dem Haushaltseinkommen auch die Beteiligungsquote (siehe Tabelle 6). Personen die über ein Haushaltseinkommen von weniger als 900 Euro verfügen, d.h. de facto unter der Armutsgrenze leben, weisen eine Beteiligungsquote von 19\% auf. Ein Haushaltseinkommen von mehr als 3000 Euro hingegen geht mit einer Beteiligungsquote von $40 \%$ einher. Mangels einer differenzierten Erfassung der oberen Einkommensklassen über 3000 Euro Haushaltseinkommen kann über den gesamten Verlauf des Zusammenhangs keine Aussage getroffen werden.

51 Institut für empirische Sozialforschung $\mathrm{GmbH}$ (http://www.ifes.at/). 
Tabelle 6: Beteiligungsquote nach Haushaltseinkommen

\begin{tabular}{lc}
\hline & Beteiligungsquote (\%) \\
\hline bis 900 Euro & 19 \\
900 bis 1200 Euro & 18 \\
1200 bis 1500 Euro & 20 \\
1500 bis 1800 Euro & 26 \\
1800 bis 2250 Euro & 32 \\
2250 bis 3000 Euro & 28 \\
über 3000 Euro & 40 \\
\hline
\end{tabular}

Datengrundlage: IFES Erhebung zur Freiwilligenarbeit in Österreich 2012; gewichtet (Quelle: BMASK 2013: 18).

Ohne multivariater Kontrolle für z.B. Bildungsgrad, Geschlecht, Alter, Kinder etc. lässt sich über die Gründe des Zusammenhangs zwischen dem Einkommen und der Freiwilligenarbeit nur spekulieren. Vor dem Hintergrund der Forschungsbefunde und motivationstheoretischer Überlegungen dürfte es sich bei materiellem Wohlstand und Einkommen in Bezug auf die Freiwilligenarbeit in der Herzberg'schen Unterscheidung, eher um einen „Hygienefaktor“ als um einen „Motivator" handeln (Herzberg 1987). Diesbezüglich sind DeVoe/Pfeffer (2007) zu einem differenzierteren Ergebnis gekommen: Neben der Höhe des Einkommens hat auch die Form der Entlohnung (z.B. monatliches Gehalt versus stundenweise Bezahlung) einen Einfluss auf die Bereitschaft sich freiwillig zu engagieren (vgl. DeVoe/Pfeffer 2007): Personen die nach geleisteten Arbeitsstunden (hourly wage) entlohnt werden, dürften eher in den Äquivalenten von Zeit und Geld denken und sind in Folge seltener freiwillig tätig als Personen, die über ein (fixes) monatliches Gehalt (salary pay) verfügen.

Rotolo et al. (2010) haben die Ungleichheiten im Vermögen und den damit verbundenen ungleichen Wohnbedingungen untersucht. Personen mit Wohneigentum leisten eher Freiwilligenarbeit als Personen, die in Miete leben, wobei der Wert des Wohnobjekts dabei keinen Einfluss auf das Ausmaß des Engagements hat. Der Unterschied wird dadurch erklärt, dass Personen, die in Eigentum leben, lokal und sozial verwurzelter sind. Personen, die in Miete leben, und dadurch auch häufiger ihren Wohnort wechseln (müssen) weisen hingegen ein geringeres Interesse an ihrem sozialen Umfeld (stake in society) auf und sind seltener freiwillig engagiert. Trotz der diesbezüglichen Unterschiede zwischen den USA und Europa sind vor dem Hintergrund der aktuellen Preisentwicklungen am (österreichischen) Immobilienmarkt sowie der Zunahme an befristeten 
Mietverträgen die Erkenntnisse von Rotolo et al. (2010) auch für die Freiwilligenarbeit in Europa von Bedeutung.

Als weitaus abgesichert gilt somit, Freiwilligenarbeit muss man sich leisten können, d.h. man muss über ein Mindestmaß an ökonomisch abgesicherte, freie Zeit verfügen. Damit ist Freiwilligenarbeit zu jenen Tätigkeiten wie Hobbys, Ausbildungen etc. zu zählen, die nicht unmittelbar mit ökonomischen Profit verbundenen sind, jedoch die Investition von Zeit und Geld erfordern (vgl. Bourdieu 1992a: 72).

\section{(II) 3.2.5. Soziales Kapital}

In der Sozialkapitalforschung stellt Freiwilligenarbeit neben Mitgliedschaften und Zugehörigkeit zu sozialen Netzwerken (vgl. Bekkers 2011; Jung/Kwon 2011; Uslaner/Brown 2008) einen zentralen Indikator für gesellschaftliche Teilhabe dar. Die zentrale Frage im Kontext des ungleichen Zugangs ist, ob die Selektions- oder die Sozialisationseffekte überwiegen, d.h. ob soziales Kapital primär Ergebnis oder aber Voraussetzung von Freiwilligem Engagement ist. Bislang konnten diese beiden Effekte in der empirischen Forschung nur teilweise entkoppelt werden (vgl. Braun 2007: 228). Obwohl durch Freiwilligenarbeit auch eine weitere Akkumulation von Kontakten und die Erweiterung der Netzwerke angenommen werden kann, ist soziales Kapital primär Voraussetzung und nicht Ergebnis der Freiwilligenarbeit, d.h. kurzgesprochen, die Selektionseffekte beim Zugang zur Freiwilligenarbeit überwiegen diesbezüglich (vgl. van Ingen 2009; van Ingen/Kalmihn 2010): „[O]nce selection effects were taken into account, we did not find a general membership effect on social resources and the effect of volunteering was small“ (van Ingen/Kalmihn 2010: 505).

Freiwilligenarbeit wird zunehmend projektorientiert, d.h. für einen begrenzten Zeitraum geleistet (vgl. Hustinx/Lammertyn 2003). Trotz dieser Beobachtung ist mit Freiwilligem Engagement weiterhin eine sozial bedeutsame Einbettung in relative homogene soziale Netzwerke über die gesamte Lebensspanne verbunden (vgl. McPherson et al. 2001).

„While voluntary groups are probably less important source of ties than school or work, they are important because they operate over the entire life course, from childhood to death, and because the represent a unique arena for watching the strong interplay of structurally induced and choice-produced homophily" (McPherson et al. 2001: 432).

Zusammenfassend belegt der Stand der empirischen Forschung zu den sozialen Merkmalen, dass abgesehen von anekdotischen Evidenzen die Freiwilligenarbeit selbst kaum zur gesellschaftlichen Inklusion benachteiligter Gruppen beitragen dürfte, sondern Exklusionseffekte und Ungleichheiten reproduziert oder aber in 
Teilbereichen noch verstärkt werden. Der sozial ungleiche Zugang zur Freiwilligenarbeit, das Zusammenspiel der Akteure, d.h. der Organisationen und der Personen, in den Feldern des Freiwilligen Engagements, werden nur selten im Zusammenhang mit den Konsequenzen in den Blick genommen. Aus diesem Grund folgt als nächstes ein Überblick zum Stand der Forschung zu den individuellen Auswirkungen bzw. Effekte der Freiwilligenarbeit.

\section{(II) 4. Stand der Forschung zu den Auswirkungen der Freiwilligenarbeit}

Neben der Bedeutung sozio-ökonomischer und sozio-demographischer Determinanten für den Zugang zur Freiwilligenarbeit sind es vor allem Studien zu den Auswirkungen der Freiwilligenarbeit, die wichtige Hinweise auf eine mögliche (Re-)Produktion sozialer Ungleichheit liefern. In welchen Dimensionen die Auswirkungen der Freiwilligenarbeit auf die Freiwilligen vermutet und untersucht werden, ist höchst unterschiedlich. Grundsätzlich ist dazu anzumerken, dass bislang nur die wenigsten Studien zu den Effekten der Freiwilligenarbeit um Selektionseffekte bereinigt sind und somit kausale Wirkrichtungen nur in den aller wenigsten Studien empirisch nachgewiesen sind. Dieses Problem besteht allerdings schon seit mehr als 40 Jahren. Smith schreibt (1975) zum Stand der Forschung zu den Effekten von Voluntary Association Participation (VAP): „However, non of these studies involed time series data that could be used to demonstrate causal linkage, so the hypothesized ,impact' of VAP is still in doubt" (264). Hier gilt es in Zukunft, wie bereits in einigen Studien im Ansatz vollzogen, die moderierenden und mediierenden Einflüsse der sozialen, askriptiven wie erworbenen, Merkmale auf den Zusammenhang zwischen dem Zugang zur Freiwilligenarbeit und der Auswirkungen der Freiwilligenarbeit zu untersuchen.

Musick/Wilson (2008) untergliedern die Studien zu den Konsequenzen der Freiwilligenarbeit in

1. Studien, die sich mit den Auswirkungen auf prosoziales Verhalten wie Einstellungen und auf die gesellschaftliche Partizipation im Allgemeinen (citizenship) beschäftigen, sowie in

2. Studien, die die Effekte auf den Beruf, das Einkommen sowie die Gesundheit und die Lebenszufriedenheit untersuchen (vgl. 459ff).

Erlinghagen (2003: 750ff) wiederum fasst die Erträge freiwilligen und ehrenamtlichen Engagements zu drei sogenannten Kuppelprodukten zusammen, die folgende Dimensionen umfassen: 
1. Reputation (z.B. „Signaling“ von sozialer Kompetenz und „Redlichkeit“),

2. weak ties (z.B. „Informationsvorsprung“ durch schwache soziale Bindungen) und

3. Humankapital (z.B. Teamfähigkeit, Lernfähigkeit, Flexibilität, etc.).

In diesem Zusammenhang weist er darauf hin, dass eine bestimmte Ausstattung an weak ties (vgl. Granovetter 1973, 2005) und Humankapital nicht nur ein Ergebnis, sondern eine Voraussetzung für Freiwilliges Engagement darstellen (ebd.: 753).

Handy/Mook (2011: 413) wiederum führen positive Auswirkungen von Freiwilligenarbeit u.a. in folgenden Bereichen an:

1. Die Zunahme an sozialem Kapital (auch von Migrantinnen und Migranten),

2. die Verbesserung der mentalen wie physischen Gesundheit, vor allem bei älteren Personen,

3. der Erwerb, das Training und die Erweiterung von Fähigkeiten und Fertigkeiten (Humankapital),

4. das Aussenden von positiven Signalen (signalling theory vgl. Spence 1973) an den Arbeitsmarkt (Wiedereinstieg, Karriere, Gehaltshöhe) oder an höhere Bildungsinstitutionen (vgl. Resumébuilding von Studierenden (Handy et al. 2010)).

Die positiven Auswirkungen variieren dabei je nach Person und nach Art und Umfang der Freiwilligenarbeit (ebd.). Der Statusgewinn stellt vor allem für ehrenamtliche Vorstands- und Gremienfunktionen einen zentralen Faktor dar (vgl. Handy et al. 2010: 413).

Die nachfolgende Darstellung der empirischen Ergebnisse trennt nach Effekten auf (1) die Erwerbstätigkeit, den Beruf und das Einkommen, sowie (2) auf die Gesundheit und die Lebenszufriedenheit sowie ergänzend die gesamtgesellschaftliche Ebene.

\section{(II) 4.1.1. Effekte auf Erwerbstätigkeit, Beruf und Einkommen}

Obwohl die positiven Effekte von Freiwilligem Engagement auf die employability immer wieder vor allem von politischer Seite hervorgehoben werden, gibt es kaum Studien, die sich mit diesem Zusammenhang im Detail beschäftigen (vgl. Nichols/Ralston 2011). Strauß (2009) belegt in ihrer ländervergleichenden Studie (Großbritannien und Deutschland) zum Einfluss von Freiwilligem Engagement auf die Wiederbeschäftigungschancen von (Langzeit-)Arbeitslosen, dass mögliche positive Effekte vom Wohlfahrtsstaatsmodell und dabei insbesondere vom Modell der Arbeitslosenversicherung und vom 
Bildungssystem abhängen. Strauß (2009) argumentiert diesen Zusammenhang folgendermaßen:

„Das Ergebnis, dass ehrenamtliches Engagement nur in Großbritannien, nicht aber in Deutschland einen positiven Effekt auf die Wiederbeschäftigungschancen von Arbeitslosen hat, legt die Vermutung nahe, dass der Erwerb von Sozialkapital durch freiwilliges Engagement in Vereinen und Verbänden einen größeren Einfluss auf die Arbeitsmarktchancen in Ländern hat, die aufgrund niedriger Arbeitslosentransfers die weniger kostenintensive informelle Arbeitssuche mit Hilfe von durch ehrenamtliches Engagement zustande gekommenen sozialen Kontakten unterstützt. Es ist ferner zu vermuten, dass in diesen Ländern ehrenamtliches Engagement als Signal für nicht-zertifizierte, in freiwilligem Engagement erworbene kognitive und nicht-kognitive Fertigkeiten eine größere Rolle spielt als in Ländern wie Deutschland, in denen eine standardisierte berufliche Ausbildung das entscheidende Signal für Arbeitgeber ist“ (662f).

Handy et al. (2010) haben in einem Ländervergleich untersucht, ob Studierende, die primär aus dem Motiv des résumé building, d.h. um den Lebenslauf für Arbeitgeber attraktiver zu gestalten und damit primär die eigene Karriere zu befördern, mehr Freiwilligenarbeit leisten als Studierende mit vordergründig altruistischen oder sozialen Motiven. Gesamt betrachtet kommt das Autorenteam dabei zu dem Ergebnis, dass Studierende mit utilitaristischen Motiven des résumé buildings nicht mehr Freiwilligenarbeit leisten als Studierende mit anderen Motiven. Sie tun dies jedoch, wie erwartet, in einer geringeren Intensität (z.B. Stunden pro Monat). Differenziert nach Länder hat sich gezeigt, dass in jenen Ländern in denen Freiwilligenarbeit eine positive Signalwirkungen auf den Arbeitsmarkt zugeschrieben wird, die Beteiligungsquoten, unabhängig von der Motivlage, höher sind (vgl. Handy et al. 2010).

Neben dem Erwerb von beruflichen Qualifikationen ist letztlich von Interesse, ob Freiwilligenarbeit auch zu einem höheren Einkommen im Beruf führt. Die Kausalität ist aufgrund der wenigen Panelstudien bis dato nicht eindeutig geklärt. Sofern nachgewiesen, wird der Zusammenhang vielfach von anderen Merkmalen wie dem Geschlecht (vgl. Day/Devlin 1996, 1998) und dem Bildungsgrad moderiert (vgl. Musick/Wilson 2008). Betreffend den moderierenden Effekt des Geschlechts vermuten Day/Devlin (1997), dass das überwiegende Engagement von Frauen in religiösen Bereichen eher negative Gewinne am Arbeitsmarkt nach sich zieht. Ökonomen versuchen den Zusammenhang zwischen Einkommen und Freiwilligenarbeit anhand zweier Modelle rationaler Entscheidungen zu erklären (vgl. Menchik/Weisbrod 1987). Aus Sicht des consumption model sind mit Freiwilligenarbeit Opportunitätskosten verbunden, die dazu führen, dass mit steigendem Einkommen das zeitliche Ausmaß an Freiwilligem Engagement sinkt, ceteris paribus Freiwilligenarbeit und Erwerbsarbeit als Substitute 
betrachtet werden. Das investment model, hingegen argumentiert, dass sich die Akkumulation von Humankapital (z.B. job skills) wie z.B. die in der Freiwilligenarbeit erworbenen Fähigkeiten positiv auf die zukünftige Einkommenshöhe, z.B. durch ability signaling auswirken (vgl. Hackl et al. 2007: 79f). Die empirischen Ergebnisse dazu bleiben widersprüchlich. Prouteau/Wolff (2006) finden in ihren Daten aus Frankreich $(\mathrm{N}=15.441)$ keinen Beleg für das Investment Model, wohingegen Hackl et al. (2007) anhand von Daten aus Oberösterreich $(\mathrm{N}=2.536$ Haushalte) dessen empirische Evidenz nachweisen konnten. Bruno/ Fiorillo (2013) konnten auf Basis von EU-SILC ${ }^{52}$ Daten $(\mathrm{N}=15.169)$ aus dem Jahr 2006 einen positiven Einfluss von Freiwilligem Engagement auf das Jahreseinkommen in der durchschnittlichen Höhe von 3,7\% feststellen. Dem gegenüber argumentiert Musick/Wilson (2008), dass Freiwilligenarbeit nicht direkt und kausal zu höheren Einkommen führt, Freiwillige jedoch Berufe mit einem höheren Prestige innehaben: „[L] ong-term volunteers select into occupations that score relatively high on the prestige scale but do not pay particularly well“ (492). Sie erklären dies u.a. damit, dass sich Freiwilliges Engagement vor allem positiv auf das soziale Netzwerk und dabei vor allem auf die weak ties (vgl. Granovetter 1973, 2005) auswirkt und infolge dazu beiträgt, „bessere“ Jobs zu finden, anschließend aber keinen Einfluss mehr auf die Einkommenshöhe hat (ebd.). Das Fazit von Musick/Wilson laut:

„First, more research is needed on the mechanism whereby volunteering leads to better Jobs [...] Second, not every kind of volunteer work brings economic benefits [...] Third, it is unclear how many people actually use the 'investment model' when choosing whether to volunteer or what to volunteer for" (2008.: 492f).

Ruiter/De Graaf (2009) kommen auf Basis der Lebenslaufdaten des Family Survey of the Dutch Population 2000 (FSDP2000), zu dem Ergebnis, dass die Einbindung (Mitgliedschaft, Freiwilligenarbeit) in Freiwillige Vereinigungen mit positiven sozio-ökonomischen Effekten betreffend des Berufsprestiges (ISEI) und der Gehaltshöhe verbunden ist. Sie nehmen dabei auch den Einfluss der sozialen Zusammensetzung der jeweiligen Freiwilligen Vereinigungen in den Blick.

"Another important finding relates to the composition of the voluntary association. If someone joins an association with more high-status co-members, this increases the likelihood to find a news job with higher status. Furthermore, joining associations with

52 EU-SILC - Community Statistics on Income an Living Conditions: Eine jährliche europaweite Befragung von Privathaushalten zum Einkommen und den Lebensbedingungen. Siehe auch: epp.eurostat.ec.europa.eu. 
relatively many co-members in supervising positions seems to lead to better paid jobs“ (Ruiter/De Graaf 2009:438).

Diese positiven Auswirkungen schreiben Ruiter/De Graaf (2009) primär dem sozialen Kapital zu und dabei ebenfalls dem Mechanismus der weak ties. Gegen das Argument, dass diese Auswirkungen auf den Erwerb von zusätzlichen Kompetenzen und Fertigkeiten, d.h. job-related-skills, beruhen, spricht der Befund, dass Freiwilligenarbeit gegenüber einfacher Mitgliedschaft nicht mit zusätzlichen Effekten verbunden ist (vgl. Ruiter/De Graaf 2009: 439).

„However, the fact that we do not find additional payoffs of volunteering (just being a member seems to suffice to reap socio-economic benefits of voluntary association involvement) seems more in line with the social network argument than with the skills argument" (Ruiter/De Graaf 2009: 439).

Für die vorliegende Arbeit von besonderer Bedeutung ist das Ergebnis, dass sich die untersuchten positiven Effekte zwischen statushöheren und statusniedrigeren Personen grundsätzlich nicht voneinander unterscheiden. „We could show that the effect of the voluntary association involvement is the same for low-status and high-status people" (Ruiter/De Graaf 2009: 438). Durch das Hinzufügen von Interaktionseffekten hinsichtlich der sozialen Herkunft wurde jedoch sichtbar, dass die positiven Effekte vom beruflichen Status des Vaters abhängen. Somit differenzieren Ruiter/De Graaf (2009) ihre Conclusio folgendermaßen:

„It indicates that people from lower status backgrounds do not gain from volunteering, whereas people from higher status backgrounds do gain occupational status if they volunteer for a voluntary association when entering the labor market" (Ruiter/De Graaf 2009: 438).

In diesem Kontext kommen van Houten et al. (2013) zu dem Befund, dass die Mitgliedschaft in Freiwilligenvereinigungen bei der intergenerationalen Übertragung des Berufsstatus zunehmend eine Rolle spielen dürfte und vor allem in statushöheren Familien eine kompensatorische Funktion bei der Statusübertragung erfüllen dürfte.

"Combined with the finding that the direct effect of parental occupational status on the [sic] that of their child has decreased over time, we conclude that social capital, defined here by voluntary association membership, is used more and more by higher status groups as a compensatory resource in the status attainment process" (van Houten et al. 2013: 25).

Gesamt betrachtet hat sich gezeigt, dass die Ergebnisse zum vielfach positiven Einfluss von Freiwilligenarbeit auf die Einkommenshöhe bzw. den Berufsstatus zumindest ansatzweise auf eine Reproduktion ökonomischer und symbolischer Dimensionen sozialer Ungleichheit durch Freiwilliges Engagement hindeuten. 


\section{(II) 4.1.2. Effekte auf Gesundheit und Lebenszufriedenheit}

Abgesehen von Einkommen, Berufsstatus und Bildungsgrad ist Gesundheit eine zentrale Dimension sozialer Ungleichheiten (vgl. Hradil 2005; Wilkinson/Pickett 2009). Die gesundheitlichen Auswirkungen von Freiwilligem Engagement werden in der Forschung primär im Kontext der gesellschaftlichen (Über-)Alterung diskutiert (vgl. Backes 2005; Caro 2008; Erlinghagen 2008; Erlinghagen/ Hank 2008; Hank/Stuck 2008; Huth 2002; Meyer et al. 2009; Mühlpfordt 2006; Olk 2002; Schroeter 2006; Wahrendorf/Siegrist 2008) und reichen bis hin zu geringeren Mortalitätsrisken von Freiwilligen (vgl. Konrath et al. 2012). Dass Freiwillige im Altersvergleich einen besseren gesundheitlichen Zustand aufweisen, wurde wiederholt in Studien bestätigt (vgl. Lum/Lightfoot 2005). Eine aktuelle Studie von Konrath et al. (2012) konnte ein geringeres Mortalitätsrisiko für jene Freiwilligen nachweisen, die außenorientierte, nicht jedoch für jene, die selbstorientierte Freiwilligenarbeit leisten. Burr et al. (2011) untersuchten wiederum den Zusammenhang von Freiwilligenarbeit mit systolischem und diastolischem Bluthochdruck. Freiwillige haben ein geringeres Risiko, an Bluthochdruck zu erkranken, jedoch nur bei moderater und nicht bei hoher zeitlicher Einbindung in die Freiwilligenarbeit. Es kommt daher auch bei der Freiwilligenarbeit nicht nur auf das Was sondern auch auf das Wie an. Von Interesse sind daher vor allem die Mechanismen, die für diesen positiven Effekt verantwortlich sind. Dabei spielen etwaige bedeutsame Moderatorvariablen, wie z.B. unterschiedliche Formen praktizierter Religiosität eine Rolle (vgl. McDougle et al. 2013).

Darüber finden sich Studien die sich auf einer allgemeineren Ebene mit Fragen des Zusammenhangs von Lebenszufriedenheit, Wohlbefinden und Vertrauen in die Gesellschaft mit Freiwilligenarbeit befassen (vgl. Fiorillo 2012; Howard/Gilbert 2008; Mojza et al. 2010; Wallace/Pichler 2009). Mojza et al. (2010) kommen in ihrer Studie zur Bedeutung von Freiwilligenarbeit bei der täglichen Erholung nach der Arbeit zu folgendem Ergebnis:

„Results from hierarchical linear modeling ( $n_{-} 529$ days) showed volunteer work during leisure time to be positively related to mastery experiences and community experiences suggesting volunteer work to contribute to successful recovery by creating new resources" (Mojza et al. 2010).

Es sei noch darauf hingewiesen, dass neben den Forschungsarbeiten zu den Auswirkungen der Freiwilligenarbeit auf individueller Ebene zahlreiche Arbeiten zu den gesamtgesellschaftlichen Effekten existieren. Diese beschäftigen sich z.B. mit der demokratiefördernden Wirkung (vgl. Hustinx et al. 2010a; Mackerle-Bixa et al. 2009; Prein et al. 2009; van Deth 2004; Verba et al. 2002) und der ökonomischen Bedeutung der Freiwilligenarbeit für die Gesellschaft (vgl. Salamon et al. 
2011) oder für einzelne Organisationen (Bowman 2009). Zum Beispiel führt ein gesamt gesehen geringeres Engagement, so argumentiert auch Putnam (1995: 167), zu einem Abbau von Sozialkapital (Vertrauen, Normen, Netzwerke) und bei jenen Gruppen mit geringerem Engagement zu einem Verlust der Fähigkeit, dieses auf andere Bereiche der Gesellschaft zu übertragen (Braun 2007: 202).

Ausgehend von den theoretischen Erörterungen in Kapitel (1) und vor dem Hintergrund des Standes der Forschung in Kapitel (II) widmet sich das Kapitel (III) nun den eigenen empirischen Analysen zur sozialen Schließung und Hierarchisierung in der Freiwilligenarbeit in Österreich. 


\section{(III) Empirische Analysen zur sozialen Schließung und Hierarchisierung in der Freiwilligenarbeit in Österreich}

\section{(III) 1. Fragestellungen für die empirischen Analysen}

Aus dem Stand der Forschung sowie den aus den aktuellen Ansätzen der Ungleichheitstheorie und der Theorie Bourdieus abgeleiteten Überlegungen lassen sich nachfolgende Fragestellungen für die Analyse der empirischen Daten zur Freiwilligenarbeit in Österreich ableiten.

\section{Soziale Schließung}

Welchen Einfluss haben askriptive wie erworbene soziale Merkmale (Geschlecht, Alter, ethnische Herkunft, Bildungsgrad, Vermögen, berufliche Stellung und Prestige) auf den Zugang zur Freiwilligenarbeit in Österreich?

\section{Hierarchisierung}

a) Welchen Einfluss haben askriptive wie erworbene soziale Merkmale (Geschlecht, Alter, ethnische Herkunft, Bildungsgrad, Vermögen, berufliche Stellung und Prestige) auf den Zugang zu Positionen mit Leitungsfunktion in der Freiwilligenarbeit in Österreich?

b) In welcher Form kommt es in der Freiwilligenarbeit zu einer Reproduktion von Prestige und Status der Erwerbsarbeit?

\section{Soziale Schließung und hierarchische Strukturierung in den Feldern der Freiwilligenarbeit}

a) Welche askriptive wie erworbene soziale Merkmale beeinflussen den Zugang zur Freiwilligenarbeit in den ausgewählten gesellschaftlichen Feldern (Soziales; Religion; Sport; Rettungsdienste)?

b) Mit welchen askriptiven wie erworbenen sozialen Merkmalen und Kapitalien korrespondiert der Zugang zu den unterschiedlichen Aufgabenbereichen und hierarchischen Positionen in den jeweiligen Feldern? 


\section{(III) 2. Methodik}

Zur Beantwortung der Fragestellungen wird auf Sekundärdaten zur Freiwilligenarbeit in Österreich aus dem Jahr 2006 zurückgegriffen. Diese Daten, die im Rahmen des Mikrozensus (4. Quartal 2006) in Form einer Zusatzerhebung erhoben wurden, stellen mit 11.661 Befragten den bis dato umfangreichsten Datensatz zur Freiwilligenarbeit in Österreich dar. Den forschungsökonomischen Vorteilen einer Sekundärdatenanalyse stehen eine Reihe an Nachteilen bzw. Einschränkungen gegenüber. Meist ist man mit dem Fehlen aus theoretischer Perspektive bedeutsamer Variablen bzw. deren ungenügender Operationalisierung konfrontiert. Dem kann vielfach nur forschungspragmatisch mit dem Verweis und dem Offenlegen der nicht in geplanter Form beantwortbaren Fragestellungen begegnet werden. Gerade jedoch für sozialstrukturelle Analysen, wie jene in der vorliegenden Arbeit, ist der Rückgriff auf bestehende Bevölkerungsstatistiken beinahe unumgänglich. Eine eigene Primärerhebung, die dem Umfang eines Dissertationsprojektes entsprechen würde, würde betreffend der Repräsentativität und damit der Verallgemeinerung der Ergebnisse deutlich hinter der Analyse von Sekundärdaten der amtlichen Statistik zurückbleiben. Vor diesem Hintergrund werden die Limitationen der Ergebnisse transparent gemacht und die Nachteile in Kauf genommen.

\section{(III) 2.1. Quantitative empirische Freiwilligenforschung}

Die empirische Erfassung von Freiwilligem Engagement hat in Europa ihren Beginn in den 1980er Jahren und ist, bis auf wenige Ausnahmen, durch quantitative Querschnittserhebungen geprägt ${ }^{53}$. Das Ziel der meisten Erhebungen war es bis dato, das Phänomen der Freiwilligenarbeit in seiner gesellschaftlichen und ökonomischen Bedeutung zu erfassen. Mittels deskriptiv ausgerichteter, repräsentativer Stichprobenuntersuchungen wird erfasst, ob die Befragten freiwillig tätig sind, und wenn ja, in welchen Bereichen, auf welche Art und in welchem zeitlichem Ausmaß. Die so erhobenen Daten werden mittels statistischer Hochrechnungs- und Gewichtungsverfahren zu Beteiligungsquoten (z.B. Anteil der Freiwilligen an der Bevölkerung) aggregiert. Das Beteiligungsausmaß, d.h. die von den Freiwilligen in einem bestimmten Referenzzeitraum, d.h. in der letzten Woche, Monat oder Jahr geleisteten Stunden werden aufsummiert als Volumen bezeichnet. Die Ermittlung des Volumens und die Umrechnung in Vollzeitäquivalente ist die Basis für die (ökonomische) Bewertung der Freiwil-

53 Zur Entwicklung der Engagementforschung im deutschsprachigen Raum siehe auch Priller (2011). 
ligenarbeit. Diese wird in einigen Ländern (Kanada, Neuseeland, Frankreich) bereits im Rahmen eines Satellitenkontos ${ }^{54}$ für den Nonprofit Sektor systematisch in die Volkswirtschaftliche Gesamtrechnung (VGR) mit einbezogen. Diese Daten sind vor allem für Ökonomen und in weiterer Folge für politische Akteure von Interesse. Ein dritter, vor allem aus soziologischer Sicht bedeutsamer Indikator ist die Beteiligungsstruktur, d.h. die Zusammensetzung der Freiwilligen nach sozio-demografischen und -ökonomischen Merkmalen. Neben den nationalen, europäischen sowie internationalen Erhebungen existieren noch eine Fülle an regionalen und bereichsspezifischen Erhebungen sowie eine Vielzahl an qualitativen Studien die sich mit der Beschreibung und den Veränderungen abseits der oben beschriebenen quantitativ messbaren Indikatoren beschäftigen, haben jedoch oftmals den Nachteil fehlender Vergleichbarkeit und Verallgemeinerbarkeit

In Österreich fand die erste umfangreiche quantitative Erhebung im Jahr 1982 statt (vgl. Badelt 1985) und umfasste, im Unterschied zu Erhebungen anderer Länder, damals schon die formelle und die informelle Freiwilligenarbeit. Knapp zwei Jahrzehnte danach fand im Rahmen eines universitären Forschungsprojekts die nächste Erhebung statt, ebenfalls mit dem primären Ziel, das Volumen zu ermitteln (vgl. Hollerweger 2001). Im Jahr 2006 beauftragte schließlich das Sozialministerium die Statistik Austria mit einer Zusatzerhebung zum Mikrozensus, die als Datenbasis für den ersten Freiwilligenbericht herangezogen wurde (vgl. BMASK 2009). 2012 wurde erneut eine Umfrage zur Freiwilligenarbeit vom BMASK in Auftrag gegeben. Die Erhebung wurde jedoch nicht wie 2006 von Statistik Austria als Zusatzmodul zum Mikrozensus, sondern von einem Markt- und Meinungsforschungsinstitut (IFES - Institut für empirische Sozialforschung $\mathrm{GmbH}$ ) durchgeführt. Die Stichprobe umfasst 4000 Personen und wurde im Unterschied zu der Erhebung 2006 nicht via Telefon, sondern Face-toFace durchgeführt. Erste Ergebnisse wurden in einem Studienbericht im Frühjahr 2013 veröffentlicht (BMASK 2013). Die Daten selbst waren jedoch bis zum Abschluss der vorliegenden Arbeit nicht für externe wissenschaftliche Zwecke freigegeben und damit für die eigene Analyse nicht verfügbar.

In Deutschland begann die Engagementforschung ebenfalls in den 1980er Jahren (vgl. Alscher et al. 2009b: 10-15) und führte, früher als in Österreich, zur Etablierung einer systematischen und regelmäßigen (alle 5 Jahre) Erhebung

54 Ein Satellitenkonto ermöglicht es, Nicht-Marktaktivitäten in das System der VGR zu integrieren und ihre ökonomische Bedeutung darzustellen (vgl. United Nations Department of Economic and Social Affairs Statistics Division 2003). 
der Freiwilligenarbeit, dem sogenannten Freiwilligensurvey (1999, 2005, 2009; vgl. Gensicke/Geiss 2010). Darüber hinaus liefern in Deutschland zahlreiche andere Studien (z.B. Engagementatlas 2009, ALLBUS, Zeitbudgetstudien, Freizeit Monitor), bereichsspezifische Erhebungen (z.B. Sportentwicklungsbericht 2007/2008) und Längsschnitterhebungen $\left(\mathrm{SOEP}^{55}\right)$ zusätzliche Daten zum Engagement (vgl. Priller 2011: 26ff.).

In der Schweiz existiert seit 1997 im Rahmen der jährlichen „Schweizer Arbeitskräfteerhebung“ (SAKE) ein Modul zur „unbezahlten Arbeit" und darin enthalten auch zur Freiwilligenarbeit. Dieses wurde bislang in den Jahren 1997, 2000, 2004, 2007 und 2010 eingeschaltet (vgl. Bundesamt für Statistik (BFS) 2011). Als Ergänzung dazu gibt es seit 2006 (alle drei Jahre) den sogenannten Freiwilligen-Monitor Schweiz der SGG ${ }^{56}$ (Stadelmann-Steffen et al. 2007; Stadelmann-Steffen et al. 2010).

Bei europaweiten Erhebungen wie dem ESS (European Social Survey) wurde Freiwilliges Engagement nur einmal im Rahmen jährlich wechselnder Fragebogenmodule in die Erhebungswelle 2002/03 eingebunden, bislang jedoch nicht als Standardmodul etabliert. Im Rahmen der (EVS) European Value Study werden zwar seit $1981(1990,1999,2008)$ Daten zur unbezahlten Arbeit erhoben, jedoch variieren dabei die abgefragten Engagementbereiche, sodass kein durchgängiger Vergleich der Beteiligungsquoten zwischen den Erhebungswellen möglich ist (European Values Study and GESIS Data Archive for the Social Sciences 2011). Weitere Daten zur Freiwilligenarbeit finden sich z.B. auch im Survey of Health, Ageing and Retirement in Europe (SHARE 2007).

Zusammengefasst liefern die verschiedenen Studien mit ihren unterschiedlichen Definitionen, Forschungszielen, Erhebungsinstrumenten und -methoden (z.B. Face-to-Face, Telefonbefragung, Onlinebefragungen, freiwillige versus verpflichtende Teilnahme) wenig international vergleichbare Daten. Das liegt u.a. auch daran, dass die Erhebungsfragen in Art, Detaillierungsgrad sowie die Referenzzeiträume zwischen den Studien vielfach stark variieren (vgl. Dekker 2011: 252ff). Zum Beispiel liegen die Beteiligungsquoten für Deutschland bei unterschiedlichen Studien (Erhebungen im Zeittraum zwischen 2001 und 2008) zwischen $18 \%{ }^{57}$ und $52 \%{ }^{58}$ (vgl. Alscher et al. 2009a: 23). Lediglich Längsschnittdaten, oder aber Querschnitterhebungen die die Erhebungsmethode und -instrument konstant halten (z.B. Freiwilligensurvey, Freiwilligen-Monitor), lassen grobe Trends erkennen. So zeigt sich, dass die Engagementquote in Deutschland

55 Sozio-ökonomisches Panel, jährliche Erhebung seit 1984.

56 Schweizer gemeinnützige Gesellschaft (http://www.sgg-ssup.ch/).

57 AWA (Institut für Demoskopie Allensbach) 2008.

58 Eurobarometer 2006. 
zwischen den Jahren 1999 - 2004 - 2009 mit 34\% - 36\% und 36\% kaum variiert (vgl. Gensicke/Geiss 2010). In diesem Kontext ernüchtern die Befunde von Dekker (2011: 253). Er konnte anhand der Analyse der Verlaufskurven verschiedener, teilweise parallel laufenden Längsschnitterhebungen in den Niederlanden, die ebenfalls die Freiwilligenarbeit erfasst haben, nachweisen, dass sich kein einheitlicher Entwicklungstrend erkennen lässt und somit beim Vergleich von Beteiligungsquoten in hohem Ausmaß mit Ehrhebungsverzerrungen und damit Methodenartefakten zu rechnen ist. Aus der jeweiligen (zeitlichen) Veränderung von Beteiligungsquoten Schlüsse für politische Interventionen abzuleiten ist daher als höchst problematisch einzustufen.

Die jeweils aktuellsten verfügbaren Ergebnisse zu den nationalen Engagementquoten finden sich in den Berichten der zuständigen staatlichen Institutionen (Österreich: BMASK ${ }^{59}$, Deutschland: $\mathrm{BMFSF}^{60}$, Schweiz: $\mathrm{EDI}^{61}$ ) und der statischen Ämter (vgl. auch die jeweiligen Engagementberichte BMASK 2009; Gensicke/Geiss 2010; Stadelmann-Steffen et al. 2010).

In Deutschland, Österreich und der Schweiz (siehe Tabelle 7) zeigen sich Ähnlichkeiten in Bezug auf die Bereiche, für die sich Menschen freiwillig engagieren. An vorderster Stelle steht in allen drei Ländern die Freiwilligenarbeit in den Bereichen Sport, Freizeit und Kultur, sowie jene in religiösen bzw. kirchennahen Vereinigungen. Der in Österreich in Relation auffällig hohe Anteil an Freiwilligen im Bereich der Katastrophenhilfs- und Rettungsdienste (6,0\%) ist zum Teil durch die Organisationsform der Freiwilligen Feuerwehren im ländlichen Raum sowie den vielfach durch Freiwillige getragenen Rettungsdienst erklärbar. In Deutschland engagieren sich im Verhältnis wiederum auffällig viele Personen im Bereich der Kindergärten und Schulen (6,9\%). Die Bereiche Umwelt- bzw. Naturschutz und, sofern erhoben Tierschutz, weisen geringere Engagementquoten auf, liegen aber in Österreich z. B. noch vor dem Bereich der Bildung. Die Tabelle 7 zeigt auch, dass trotz eines bestehenden internationalen Klassifikationsschemas $\left(\mathrm{ICNPO}^{62}\right)$ von Drittsektororganisationen bis dato keine länderübergreifend einheitliche Erhebung der Bereiche bzw. Engagementfelder existiert und damit eine differenzierte Vergleichbarkeit weiterhin eingeschränkt bleibt.

59 Bundesministerium für Arbeit, Soziales und Konsumentenschutz.

60 Bundesministerin für Familie, Senioren, Frauen und Jugend.

61 Eidgenössisches Departement des Innern.

62 International Classification of Nonprofit Organizations (ICNPO). 
Tabelle 7: Beteiligungsquoten formeller Freiwilligenarbeit nach Bereichen in Österreich, Deutschland und der Schweiz

\begin{tabular}{|c|c|c|c|c|c|}
\hline \multicolumn{2}{|c|}{$\begin{array}{c}\text { Österreich } \\
\text { (Rameder/More- } \\
\text { Hollerweger 2009: 52) }\end{array}$} & \multicolumn{2}{|c|}{$\begin{array}{c}\text { Deutschland (2009) } \\
\text { (Gensicke/Geiss 2010: 93) }\end{array}$} & \multicolumn{2}{|c|}{$\begin{array}{c}\text { Schweiz (2010) } \\
\text { (Stadelmann-Steffen et al. } \\
\text { 2010: 167) }\end{array}$} \\
\hline Bereich & Anteil & Bereich & Anteil & Bereich & Anteil \\
\hline \multirow{2}{*}{$\begin{array}{l}\text { Kunst, Kultur, } \\
\text { Unterhaltung und } \\
\text { Freizeit }\end{array}$} & \multirow[t]{2}{*}{$7,5 \%$} & Kultur, Kunst, Musik & $5,2 \%$ & Kulturelle Vereine & $5,8 \%$ \\
\hline & & $\begin{array}{l}\text { Freizeit und } \\
\text { Geselligkeit }\end{array}$ & $4,6 \%$ & $\begin{array}{l}\text { Spiel-/Hobby-/ } \\
\text { Freizeitvereine }\end{array}$ & $5,0 \%$ \\
\hline Sport und Bewegung & $6,9 \%$ & Sport und Bewegung & $10,1 \%$ & Sportvereine & $10,1 \%$ \\
\hline \begin{tabular}{|l} 
kirchlicher oder \\
religiöser Bereich
\end{tabular} & $6,2 \%$ & Religion und Kirche & $6,9 \%$ & $\begin{array}{l}\text { Kirchliche } \\
\text { Organisationen }\end{array}$ & $4,5 \%$ \\
\hline $\begin{array}{l}\text { Katastrophenhilfe } \\
\text { und Rettungsdienste }\end{array}$ & $6,0 \%$ & $\begin{array}{l}\text { Freiwillige Feuerwehr } \\
\text { und Rettungsdienste }\end{array}$ & $3,1 \%$ & & \\
\hline \multirow[t]{3}{*}{$\begin{array}{l}\text { politische Arbeit und } \\
\text { Interessensvertretung }\end{array}$} & \multirow[t]{3}{*}{$3,5 \%$} & $\begin{array}{l}\text { Politische } \\
\text { Interessensvertretung }\end{array}$ & $2,7 \%$ & $\begin{array}{l}\text { Politische/öffentli- } \\
\text { che Ämter }\end{array}$ & $1,2 \%$ \\
\hline & & \multirow{2}{*}{$\begin{array}{l}\text { Berufliche } \\
\text { Interessensvertretung }\end{array}$} & \multirow[t]{2}{*}{$1,8 \%$} & Interessensverbände & $3,0 \%$ \\
\hline & & & & Politische Parteien & $0,9 \%$ \\
\hline \multirow{2}{*}{$\begin{array}{l}\text { Sozial- und } \\
\text { Gesundheitsbereich }\end{array}$} & \multirow[t]{2}{*}{$3,3 \%$} & Sozialer Bereich & $5,2 \%$ & \multirow{2}{*}{$\begin{array}{l}\text { Soziale/karitative/ } \\
\text { gemeinnützige } \\
\text { Organisationen }\end{array}$} & \multirow[t]{2}{*}{$4,1 \%$} \\
\hline & & Gesundheit & $2,2 \%$ & & \\
\hline $\begin{array}{l}\text { Umwelt, Natur und } \\
\text { Tierschutz }\end{array}$ & $2,6 \%$ & Natur und Tierschutz & $2,8 \%$ & $\begin{array}{l}\text { Menschenrechts-/ } \\
\text { Umweltverbände }\end{array}$ & $1,1 \%$ \\
\hline \multirow[t]{2}{*}{ Bildung } & \multirow[t]{2}{*}{$2,5 \%$} & $\begin{array}{l}\text { Jugendarbeit und } \\
\text { Erwachsenenbildung }\end{array}$ & $2,6 \%$ & & \\
\hline & & $\begin{array}{l}\text { Kindergarten und } \\
\text { Schule }\end{array}$ & $6,9 \%$ & & \\
\hline \multirow[t]{2}{*}{$\begin{array}{l}\text { Bürgerliche } \\
\text { Aktivitäten und } \\
\text { Gemeinwesen }\end{array}$} & $2,1 \%$ & $\begin{array}{l}\text { Lokales } \\
\text { Bürgerengagement }\end{array}$ & $1,9 \%$ & Öffentlicher Dienst & $2,6 \%$ \\
\hline & & Kriminalitätsprobleme & $0,7 \%$ & & \\
\hline Gesamt $^{\mathrm{a}}$ & $27,9 \%$ & Gesamt $^{\mathrm{a}}$ & $36,0 \%$ & Gesamt $^{a}$ & $26,1 \%$ \\
\hline
\end{tabular}

Gesamtwerte bezeichnen die jeweilige Beteiligungsquote ohne Mehrfachnennungen (vgl. More-Hollerweger/Rameder 2013: 388). 
Rund um das Thema der Lückenbüßerfunktion der Freiwilligenarbeit für (sozial-)staatlichen Rückzug wird das Potential des Freiwilligen Engagements vielfach mit Bezug auf die Gesamt-Beteiligungsquote von z.B. in Österreich 27,9\% und nicht auf Basis der rund 9,3\% der Bevölkerung, die in den Bereichen Soziales und Gesundheit sowie Katastrophenhilfe (freiwillige Feuerwehren) und Rettungsdienste engagieren sind, diskutiert. Die Beteiligungsquote in diesem Bereich von 9,3\% Prozent entspricht rund 31\% aller Freiwilligen in Österreich. Zählt man noch die Freiwilligen im Bereich der Kirchen und der Religion mit einer Beteiligungsquote von 6,2\% hinzu, liegt der Anteil bei rund $50 \%$ aller Freiwilligen. Eine ähnliche Verteilung der Engagementbereiche findet sich auch in Deutschland und der Schweiz. In Deutschland sind bei einer Gesamt-Beteiligungsquote von 36\% rund 8\% der Bevölkerung im Sozial- und Gesundheitsbereich bzw. bei der Freiwilligen Feuerwehr und bei Rettungsdiensten freiwillig engagiert. In der Schweiz finden sich ähnliche Ergebnisse: 4,1\% der Bevölkerung sind als Freiwillige in sozialen, karitativen bzw. gemeinnützigen Organisationen tätig, die Gesamt-Beteiligungsquote beträgt hingegen 26,1\%. Realistischere Einschätzungen zu den Potentialen der Freiwilligenarbeit sollten sich daher künftig weniger an den Gesamt-Beteiligungsquoten orientieren, sondern der Heterogenität (Bereiche/Felder) und der Kontextabhängigkeit (Stadt/ Land) der Freiwilligenarbeit Rechnung tragend, feldspezifischen Analysen als Beurteilungsbasis heranziehen.

Abschließend sei noch die persönliche Einschätzung erwähnt, dass die Inhalte und die Indikatoren der amtlichen bzw. quasi-amtlichen großen statistischen Erhebungen zur Freiwilligenarbeit primär an deren ökonomischer Bewertung ausgerichtet sind. Als Auftraggeber ist der Staat (Politik, Ministerien) vorwiegend an der Anzahl an Freiwilligen und der dabei geleisteten Arbeitsstunden interessiert. Zusätzlich werden die Beteiligungsquoten zur Beurteilung der Entwicklung im Zeitverlauf und dem internationalen Benchmark benötigt. Diese Anforderungen sind jedoch für die wissenschaftliche Forschung vielfach ungenügend. Diese benötigt genauere Daten zu den Einflussfaktoren sowie den Wechsel- und Auswirkungen auf individueller, institutioneller und gesellschaftlicher Ebene. Die staatliche Finanzierung dafür nötiger kostenintensiver Längsschnittuntersuchungen oder Panelstudien ist jedoch bis dato in Österreich ausgeblieben. Für eine vielschichtige, die positiven wie negativen Externalitäten berücksichtigende Bewertung der Freiwilligenarbeit (z.B. mittels SROI Analysen) braucht es jedoch Grundlagenforschung zu den Bedingungen und Auswirkungen der Freiwilligenarbeit. 


\section{(III) 2.2. Beschreibung des Datensatzes}

\section{Zusatzerhebung zum Österreichischen Mikrozensus 2006/4.Quartal}

Die Erhebung zur „Struktur und Volumen der Freiwilligenarbeit in Österreich“ wurde von Statistik Austria im Jahr 2006 im Auftrag des damaligen Ministeriums für soziale Sicherheit, Generationen und Konsumentenschutz durchgeführt. Die Befragung erfolgte als Zusatzmodul zur Mikrozensuserhebung im 4. Quartal 2006 in den in Tabelle 8 beschriebenen Bereichen bzw. Feldern.

Tabelle 8: Tätigkeitsbereiche der Freiwilligenarbeit

\begin{tabular}{|c|c|}
\hline Bereich & Beispiele für Organisationen bzw. Tätigkeiten \\
\hline $\begin{array}{l}\text { Katastrophenhilfs- und } \\
\text { Rettungsdienste }\end{array}$ & $\begin{array}{l}\text { Freiwillige Feuerwehren, Rettungsdienste (Rotes Kreuz, } \\
\text { Bergrettung), humanitäre Hilfsorganisationen }\end{array}$ \\
\hline $\begin{array}{l}\text { Kunst, Kultur, } \\
\text { Unterhaltung und Freizeit }\end{array}$ & $\begin{array}{l}\text { Musikkapellen, Trachtengruppen, Theater- oder } \\
\text { Tanzgruppen, Kunstvereine, Museen, Geselligkeitsvereine }\end{array}$ \\
\hline $\begin{array}{l}\text { Umwelt, Natur und } \\
\text { Tierschutz }\end{array}$ & $\begin{array}{l}\text { Natur-, Berg- und Wandervereine, } \\
\text { Tierschutzorganisationen, Umweltorganisationen, Obst- } \\
\text { und Gartenbauvereine, Tierzuchtvereine }\end{array}$ \\
\hline Kirche und Religion & $\begin{array}{l}\text { Pfarrgemeinderäte, Gremien von Religionsgemeinschaften, } \\
\text { religiöse Kinder- oder Jugendgruppen bzw. Frauen und } \\
\text { Männerbewegungen }\end{array}$ \\
\hline Soziales und Gesundheit & $\begin{array}{l}\text { Sozial- bzw. Hilfsorganisationen, Jugendzentren bzw. } \\
\text {-gruppen, Seniorenorganisationen, Selbsthilfegruppen, } \\
\text { Besuchs- oder Begleitdienste }\end{array}$ \\
\hline $\begin{array}{l}\text { Politische Arbeit und } \\
\text { Interessenvertretung }\end{array}$ & $\begin{array}{l}\text { Politische Initiativen oder Vereine, Gemeinde- oder } \\
\text { Stadträte, Menschenrechts- oder Solidaritätsprojekte, } \\
\text { Organisationen der Entwicklungshilfe }\end{array}$ \\
\hline $\begin{array}{l}\text { Bürgerliche Aktivitäten } \\
\text { und Gemeinwesen }\end{array}$ & Bürgerinitiativen, BürgerInnenbüros, Nachbarschaftszentren \\
\hline Bildung & $\begin{array}{l}\text { Elternvereine, SchülerInnenvertretungen, } \\
\text { Erwachsenenbildungseinrichtungen, Lernhilfezentren, } \\
\text { Bibliotheken }\end{array}$ \\
\hline Sport und Bewegung & $\begin{array}{l}\text { Sport- oder Turnvereine, Fachverbände oder sonstige } \\
\text { Sportorganisationen, Bewegungsgruppen }\end{array}$ \\
\hline $\begin{array}{l}\text { Nachbarschaftshilfe und } \\
\text { informelle ehrenamtliche } \\
\text { Tätigkeiten }\end{array}$ & $\begin{array}{l}\text { Tätigkeiten für Personen außerhalb des eigenen Haushalts: } \\
\text { Hausarbeiten (Einkaufen, Blumen gießen), Reparaturen, } \\
\text { Besuche betreuungsbedürftiger bzw. pflegebedürftiger } \\
\text { Personen, Fahrtendienste, Gartenpflege, Schriftsachen und } \\
\text { Amtswege, private unbezahlte Nachhilfe }\end{array}$ \\
\hline
\end{tabular}

Quelle: BMASK (2009: 212ff); More-Hollerweger/Sprajcer (2009). 
Die Beantwortung der Fragen war im Gegensatz zum Grundprogramm des Mikrozensus, dessen Beantwortung verpflichtend ist, freiwillig und erfolgte ausschließlich telefonisch. Insgesamt wurden rund 63\% der Gesamtstichprobe $(\mathrm{N}=50.117)$ in der Haupterhebung $(\mathrm{N}=26.128)$ und der Nacherhebung $(\mathrm{N}=5.119)$ gebeten, zum Thema der Freiwilligenarbeit Auskunft zu geben (siehe Tabelle 9). Schlussendlich haben insgesamt 11.661 Personen den Fragebogen beantwortet, 19.586 Personen haben die Durchführung des Interviews abgelehnt.

Tabelle 9: Erhebungsdesign

\begin{tabular}{|l|l|l|l|l|}
\hline & Respondenten & Teilrespondenten & Verweigerer & insgesamt \\
\hline Haupterhebung & $\begin{array}{l}\text { 9360 Personen, } \\
\text { 63\% Freiwilligen- } \\
\text { arbeit }\end{array}$ & $\begin{array}{l}16768 \\
\text { Personen }\end{array}$ & $\begin{array}{l}26128 \\
\text { Personen }\end{array}$ \\
\hline Nacherhebung & $\begin{array}{l}\text { 2 301 Personen, } \\
\text { 51\% Freiwilligen- } \\
\text { arbeit }\end{array}$ & $\begin{array}{l}1810 \text { Personen, } \\
\text { 11\% Freiwilligen- } \\
\text { arbeit }\end{array}$ & 1008 Personen & $\begin{array}{l}5119 \\
\text { Personen }\end{array}$ \\
\hline
\end{tabular}

Quelle: Statistik Austria (2007: 8, leicht modifizierte Darstellung).

In der Haupterhebung war die Auskunftsbereitschaft mit 36\% (9.360 von 26.128) relativ gering (vgl. Statistik Austria 2007: 8). Aufgrund eines vermuteten NonResponse-Bias wurde von Statistik Austria eine Nacherhebung durchgeführt. Es hat sich dabei gezeigt, dass die Beteiligungsquote an der Zusatzerhebung zur Freiwilligenarbeit positiv mit der Beteiligungsquote an der Freiwilligenarbeit korreliert.

„Interessant ist weiters die Tatsache, dass sich in der Haupterhebung (nicht hochgerechnet) $63 \%$ als freiwillig Tätige deklariert haben, bei der Nacherhebung waren es nur mehr $51 \%$ und unter den TeilrespondentInnen der Nacherhebung gar nur 11\%. Es besteht also offensichtlich ein negativer Zusammenhang zwischen dem Ausmaß der Freiwilligenarbeit und der Ausschöpfung der Stichprobe“ (Statistik Austria 2007: 9).

Diesen Responsebias gilt es infolge bei der Interpretation und hinsichtlich der Verallgemeinerbarkeit der Ergebnisse zu berücksichtigen.

Um Aussagen über die Grundgesamtheit in Österreich treffen zu können, wurde von der Statistik Austria ein Basishochrechnungsgewicht, angepasst an Verteilungsvorgaben des Mikrozensus für das Freiwilligensample, errechnet (siehe dazu Statistik Austria 2007: 119ff). Berücksichtigt wurden das Geschlecht, das Alter, die Staatsbürgerschaft und die Erwerbstätigkeit. Da jedoch nicht für weitere, für die Freiwilligenarbeit bedeutsame Einflussfaktoren, wie z.B. den 
Bildungsgrad, die Wohnortgröße bzw. der Urbanisierungsgrad, etc. korrigiert wurde, wird für die in dieser Arbeit nachfolgenden Analysen, sofern nicht anders angegeben, auf die Verwendung des Hochrechnungsgewichts verzichtet, und damit bewusst Einschränkungen bei der Verallgemeinerbarkeit der Ergebnisse in Kauf genommen. Die in nachfolgenden Analysen dargestellten Beteiligungsquoten weichen daher von jenen aus den Berichten der Statistik Austria als auch des ersten Freiwilligenberichts zum Freiwilligen Engagement in Österreich ab und sind nicht direkt vergleichbar (vgl. BMASK 2009).

\section{(III) 2.3. Variablenbeschreibung}

Die abhängigen Variablen sind (a) der Zugang zur Freiwilligenarbeit, gemessen an den freiwilligen und ehrenamtlichen Tätigkeiten in den unterschiedlichen Bereichen, und (b) die Funktion in der Freiwilligenarbeit, gemessen an der Art der Aufgabe und Funktion im Rahmen der jeweiligen ehrenamtlichen Tätigkeiten. Als erklärende Variable fungieren jene im Datensatz vorhandenen Indikatoren für die askriptiven und erworbenen Merkmale, die als zentrale Determinanten sozialer Ungleichheit gelten. Somit wurden aus den verfügbaren Variablen des Mikrozensusdatensatzes nur jene Variablen ausgewählt, deren Einfluss auf die jeweilige abhängige Variable vor dem Hintergrund der jeweils konkreten Fragestellung sowohl theoretisch als auch auf Basis des Stands der Forschung, begründbar ist. Damit wird dem „Überladen“ der Erklärungsmodelle (vorwiegend logistische Regressionsanalysen) zur alleinigen Steigerung der Erklärungskraft (Pseudo $\mathrm{R}^{2}$ ) vorgebeugt.

\section{(III) 2.3.1. Abhängige Variablen}

\section{Zugang zur Freiwilligenarbeit}

Statistik Austria hat für jeden der neun Bereiche (siehe Tabelle 8) die ehrenamtlichen bzw. freiwilligen Tätigkeiten einzeln erhoben (siehe dazu den Fragebogen im Anhang). Der allgemeine Zugang zur Freiwilligenarbeit wurde anhand der Aggregation der Antworten auf die Fragen zu den ehrenamtlichen Tätigkeiten in den einzelnen Bereichen, gemessen.

Die Frage je Bereich lautete: „Sind Sie irgendwo im Bereich [A bis I] ehrenamtlich für eine Organisation oder einen Verein tätig? z.B. [...]“. Die Antwortmöglichkeiten umfassten jeweils Ja (1) und Nein (2). Für die weiterführenden Analysen wurden die Variablen zur Freiwilligenarbeit in einem ersten Schritt je Bereich in $\mathrm{Ja}$ (1) und Nein (0) neu kodiert, und anschließend über die Bereiche aufsummiert und wieder dichotomisiert, $\geq 1 \mathrm{Ja}$ (1) und $<1$ Nein (0). 


\begin{tabular}{lll}
\hline Gesamt & Freiwilligenarbeit & 0 nein \\
& & 1 ja \\
Je Bereich & Freiwilligenarbeit Bereich 1 bis 9 & 0 nein \\
& & $1 \mathrm{ja}$ \\
\hline
\end{tabular}

\section{Leitende Funktion in der Freiwilligenarbeit}

Für jede genannte ehrenamtliche Tätigkeit wurde auch nach der weiteren Spezifizierung der Aufgaben bzw. der Funktion gefragt: „Welche Aufgabe erfüllen Sie im Rahmen ihrer Tätigkeiten (Mehrfachantworten möglich)“. Die Antwortmöglichkeiten umfassten folgende vier vorgegebenen Kategorien:

1. Kernaufgabe der Organisation

2. Administrative/unterstützende Aufgaben

3. Leitende Funktion/FunktionärIn

4. Sonstige Aufgaben

Für die weitere Auswertung wurden diese Variablen wie folgt in dichotome Variablen neu kodiert.

\begin{tabular}{|l|l|}
\hline (1) Kernaufgabe der Organisation & (0) Kernaufgabe/administrative/sonstige \\
\cline { 1 - 2 } (2) Administrative/unterstützende Aufgaben & Funktion \\
\hline (4) Sonstige Aufgaben & \\
\hline (3) Leitende Funktion & (1) Leitende Funktion \\
\hline
\end{tabular}

Für die weiterführenden Analysen mittels logistischen Regressionsanalysen wurden die dichotomisierten Variablen zur Aufgabe/Funktion in der Freiwilligenarbeit wieder über die Bereiche aufsummiert und anschließend erneut nach dem Schema $\geq 1 \mathrm{Ja}$ (1) und $<1$ Nein (0) dichotomisiert.

\begin{tabular}{lll}
\hline Gesamt & Leitende Funktion in der Freiwilligenarbeit & 0 nein \\
& & $1 \mathrm{ja}$ \\
Je Bereich & Leitende Funktion im Bereich 1 bis 9 & 0 nein \\
& & $1 \mathrm{ja}$ \\
\hline
\end{tabular}

Für die feldspezifischen Analysen mittels Multipler Korrespondenzanalysen (Kapitel (III) 4.4.4.) wird hingegen wieder die ursprüngliche, nicht dichotomisierte Form der Variablenausprägung verwendet. 


\section{(III) 2.3.2. Erklärende Variablen}

Die erklärenden Variablen (Tabelle 10) umfassen somit die jeweiligen Indikatoren für die askriptiven Merkmale, Geschlecht, Alter und Geburtsland sowie für die erworbenen Merkmale, Bildungsgrad, Erwerbstätigkeit, beruflicher Status (ISEI und Leitungsfunktion), Wohnrechtsverhältnis und Familienstand. Als Kontextvariable wird der Urbanisierungsgrad hinzugenommen.

Tabelle 10: Erklärende Variablen - Merkmale und Variablenausprägung

\begin{tabular}{ll}
\hline Variablen (Label) & Variablenausprägung \\
\hline $\begin{array}{l}\text { Geschlecht: } \\
\text { (bsex) }\end{array}$ & Männlich (1) \\
\hline Alter & Weiblich (2) \\
(balt) & Alter in Jahren \\
\hline (balt recoded) & Alter in Gruppen: \\
& $15-29(1)$ \\
& $30-49(2)$ \\
& $50-65(3)$ \\
\hline Geburtsland & $65+(4)$ \\
(xbgeblao) & Österreich (1) \\
\hline Staatsbürgerschaft & Nicht- Österreich (2) \\
(xbstaato) & Österreich (1) \\
\hline Bildungsgrad & Nicht Österreich (2) \\
(xkartab recoded) & Pflichtschule/keine Pflichtschule (1) \\
& Lehrabschluss (Berufsschule) (2) \\
& Berufsbild. mittlere Schule (3) \\
& AHS, BHS (4) \\
& Kolleg, Uni-LG, hochschulverwandte \\
& Lehranstalten (5) \\
\hline Erwerbstätigkeit & Universität, Fachhochschule (6) \\
(cerw recoded) & Nein (0) \\
\hline Beruflicher Status (ISEI) & Ja (1) \\
(auf Basis des ISCO-4Steller) & Punktewerte von 16 bis 90 \\
\hline Berufliche Stellung & \\
(dbers2 recoded) & Nicht erwerbstätig (0) \\
& Landwirt (1) \\
& Selbstständig (2) \\
\hline & Arbeiter (3) \\
& Angestellter (4) \\
& Führungskraft (5) \\
\hline
\end{tabular}




\begin{tabular}{ll}
\hline $\begin{array}{l}\text { Leitungsfunktion in der beruflichen } \\
\text { Tätigkeit } \\
\text { (dleit recoded) }\end{array}$ & $\begin{array}{l}\text { Nein (0) } \\
\text { Ja (1) }\end{array}$ \\
\hline Wohnrechtsverhältnis: Eigentum & Nein (0) \\
(wrecht recoded) & Ja (1) \\
\hline Familienstand: Verheiratet & Nein (0) \\
(bfst recoded) & Ja (1) \\
\hline Eurostat-Urbanisierungsgrad & Niedrige Bevölkerungsdichte (1) \\
(xurb recoded) & Mittlere Bevölkerungsdichte (2) \\
& Hohe Bevölkerungsdichte (3) \\
\hline
\end{tabular}

Quelle: Mikrozensus Zusatzerhebungen Q4/2006; eigene Darstellung.

\section{(III) 2.5. Deskriptive Statistik}

Die Tabelle 11 zeigt die deskriptive Statistik ${ }^{63}$ hinsichtlich Messniveau, gültiger Fälle, Minimum und Maximum der Variablenausprägung sowie die Mittelwerte und ggf. die Standardabweichung. Im Falle der Variable „Freiwilligenarbeit“ bedeutet z.B. ein Mittelwert von 0,413, dass 41,3\% der Befragten ( $N=11.657)$ die Frage mit Ja (1) beantwortet haben. 26,8\% (Mittelwert: 0,268) der Freiwilligen sind im Rahmen ihrer Tätigkeiten mit ehrenamtlichen Leitungsfunktionen betraut.

Ein Beispiel zu den Feldern: Der Anteil an Personen des Samples (N=11.657) die im Feld der Katastrophenhilfe freiwillig tätig sind beträgt 8,2\% (Mittelwert: 0,082). Betreffend der erklärenden Variablen zeigt sich, dass 52,8\% der Respondenten Frauen sind, das Durchschnittsalter bei 45 Jahre liegt, rund 8\% nicht in Österreich geboren sind und 64,2\% erwerbstätig sind. Knapp 70\% der Respondenten leben in Wohneigentum. Darüber hinaus sind 58,5\% verheiratet. Der Gruppe der Berufstätigen kann im Durschnitt ein ISEI Wert von 43,6 Punkten (von maximal 90 Punkten) zugewiesen werden. Bei rund 28\% der Erwerbstätigen ist die Tätigkeit mit einer Leitungsfunktion verbunden. Die durchschnittliche Wochenarbeitszeit der Erwerbstätigen beträgt 38,9 Stunden. Der Vergleich der deskriptiven Ergebnisse mit der österreichischen Bevölkerung (Grundgesamtheit) erfolgt im ersten Kapitel zu den Ergebnissen zur Sozialstruktur (Kap. (IV) 3.1 )

63 Bei dichotomen Variablen geben die Mittelwerte Aufschluss über die prozentuelle Verteilung und stellen multipliziert mit der Zahl 100 die jeweiligen Prozentwerte der Verteilung dar. 
Tabelle 11: Deskriptive Statistik zu den abhängigen und erklärenden Variablen

\begin{tabular}{|c|c|c|c|c|c|}
\hline Variablen & Messniveau & $\mathbf{N}$ (gültig) & Min & Max & Mittelwert (SD) \\
\hline Freiwilligenarbeit & dichotom & 11.657 & 0 & 1 & 0,413 \\
\hline $\begin{array}{l}\text { Katastrophenhilfsdienste } \\
\text { binär }\end{array}$ & dichotom & 11.657 & 0 & 1 & 0,082 \\
\hline Religion binär & dichotom & 11.657 & 0 & 1 & 0,092 \\
\hline Soziales binär & dichotom & 11.657 & 0 & 1 & 0,051 \\
\hline Sport binär & dichotom & 11.657 & 0 & 1 & 0,102 \\
\hline $\begin{array}{l}\text { Leitende Funktion in } \\
\text { der Freiwilligenarbeit }\end{array}$ & dichotom & 4.812 & 0 & 1 & 0,268 \\
\hline $\begin{array}{l}\text { Mehrfachengagement } \\
\text { (Organisationen) }\end{array}$ & metrisch & 4.814 & 1 & 6 & $1,701(1,088)$ \\
\hline Geschlecht (ref. Männer) & dichotom & 11.657 & 0 & 1 & 0,528 \\
\hline Alter & metrisch & 11.657 & 15 & 94 & $45,030(16,224)$ \\
\hline $\begin{array}{l}\text { Geburtsland (Ref: } \\
\text { Österreich) }\end{array}$ & dichotom & 11.657 & 0 & 1 & 0,080 \\
\hline Bildungsgrad & ordinal & 11.657 & 1 & 6 & 2,716 \\
\hline $\begin{array}{l}\text { Erwerbstätigkeit (ref. } \\
\text { Erwerbslos) }\end{array}$ & dichotom & 11.657 & 0 & 1 & 0,642 \\
\hline $\begin{array}{l}\text { Leitende Funktion im Beruf } \\
\text { (ref. nicht leitend) }\end{array}$ & dichotom & 6.431 & 0 & 1 & 0,283 \\
\hline $\begin{array}{l}\text { Sozio-ökonomischer Status } \\
\text { (ISEI) }\end{array}$ & metrisch & 7.488 & 16 & 90 & $43,575(15,990)$ \\
\hline $\begin{array}{l}\text { normale } \\
\text { Wochenarbeitsstunden }\end{array}$ & metrisch & 7.441 & 0,5 & 126 & $38,861(14,930)$ \\
\hline $\begin{array}{l}\text { Wohnrechtsverhältnis } \\
\text { Eigentum (ref. Miete) }\end{array}$ & dichotom & 11.657 & 0 & 1 & 0,698 \\
\hline $\begin{array}{l}\text { Familienstand verheiratet } \\
\text { (ref. ledig, geschieden, } \\
\text { verwitwet) }\end{array}$ & dichotom & 11.657 & 0 & 1 & 0,585 \\
\hline Bevölkerungsdichte & ordinal & 11.657 & 1 & 3 & 1,844 \\
\hline
\end{tabular}

Quelle: Mikrozensus-Zusatzerhebung 2006/Q4; eigene Berechnung.

\section{(III) 2.6. Statistische Analyseverfahren}

Die Aufbereitung und Analyse der Daten erfolgte in der Statistiksoftware IBM SPSS 20.0. Die grafische Aufbereitung der Ergebnisse in Tabellen und Diagrammen erfolgte in Microsoft EXCEL 2010. Da es in SPSS 20.0 nicht möglich ist, 
Multiple Korrespondenzanalysen durchzuführen, wurde auf das EXCEL Plugin XLSTAT in der Version 2013.3.02 zurückgegriffen.

\section{(III) 2.6.1. Deskriptive und bivariate Analyseverfahren}

Die deskriptive wie bivariate Analyse erfolgte in Abhängigkeit des Messniveaus der Variablen, bei kategorialen Variablen anhand von Kontingenztabellen (Kreuztabellen) und im Falle von metrischen Variablen, je nach Fragestellung (Zusammenhänge oder Unterschiede), mit Korrelationsanalysen oder Mittelwertvergleichen.

\section{(III) 2.6.2. Multivariate Analyseverfahren}

\section{Logistische Regressionsanalyse}

Zur multivariaten Überprüfung des Einflusses der erklärenden Variablen auf die abhängigen Variablen werden aufgrund der dichotomen Variablenausprägung logistischen Regressionsanalysen verwendet (vgl. Backhaus 2006: 425ff; Mood 2009; Pampel 2000). Die logistische Regressionsanalyse zählt zu den strukturprüfenden Verfahren (vgl. Backhaus 2006: 428) und errechnet bzw. schätzt den Einfluss der unabhängigen Variablen auf die Eintrittswahrscheinlichkeit für ein bestimmtes Ereignis (abhängige Variable). Im Gegensatz zur linearen Regressionsanalyse unterstellt die logistische Regressionsanalyse „einen nicht-linearen Zusammenhang zwischen der Eintrittswahrscheinlichkeit der binären, abhängigen Variable $[\mathrm{P}(\mathrm{y}=1)]$ und den unabhängigen Variablen als Modellprämisse“ (Backhaus 2006: 432). Dies hat vor allem Auswirkungen auf die Interpretation der Ergebnisse, d.h. konkret der Regressionskoeffizienten. Die Koeffizienten sind erstens nicht untereinander vergleichbar und die Wirkung der erklärenden Variablen ist nicht „über die gesamte Breite ihrer Ausprägung konstant“ (Backhaus 2006: 439). Interpretierbar ist die Richtung des Einflusses der erklärenden Variablen, wobei ein negativer Koeffizient (Beta) mit einer sinkenden Wahrscheinlichkeit und ein positiver Koeffizient mit einer steigenden Wahrscheinlichkeit für das Ereignis $\mathrm{y}=1$ verbunden ist. Eine Erleichterung der Interpretation der Koeffizienten (Beta) wird durch die Angabe der sogenannten Odds (Chancen) erreicht. Diese, auch als Odds Ratio $(\operatorname{Exp}(B))$ bezeichneten Werte stellen „nicht die Eintrittswahrscheinlichkeit $\mathrm{P}(\mathrm{y}=1)$ selbst [dar], sondern ihr Verhältnis zur Gegenwahrscheinlichkeit $\mathrm{P}(\mathrm{y}=0)$ bzw. 1-P $(\mathrm{y}=1)$ [...] Dieses Wahrscheinlichkeitsverhältnis spiegelt die Chance (Odd) wider, das Ergebnis $\mathrm{y}=1 \mathrm{im}$ Vergleich zum Ergebnis y=0 zu erhalten“" (Backhaus 2006: 442): 


$$
\operatorname{Odds}(y=1)=\frac{p(y=1)}{1-P(y=1)}
$$

Zur Beurteilung des Gütemaßes des Regressionsmodells (Gesamtfit) stehen unterschiedliche Pseudo-R-Quadrat-Statistiken (Cox und Schnell, McFadden oder Nagelkerke) zur Verfügung. Aufgrund seiner eindeutigeren Interpretierbarkeit (der Wert kann maximal 1 erreichen) ist dem Nagelkerke- $\mathrm{R}^{2}$ der Vorzug zu geben (vgl. Backhaus 2006: 449).

\section{Multiple Korrespondenzanalyse (MCA)}

Die multiple Korrespondenzanalyse (MCA) (vgl. Greenacre 2007; Le Roux/ Rouanet 2010) ist ein multivariates Verfahren zur grafischen Darstellung von mehrdimensionalen Kontingenztabellen. Im Vordergrund stehen die Visualisierung von Beziehungen und die Entdeckung von Strukturen zwischen kategorialen Variablen. In der Literatur findet sich eine anhaltende Diskussion über die Gefahr möglicher Fehlinterpretationen der grafisch dargestellten MCA. Grundsätzlich stellt die euklidische Distanz zwischen den einzelnen Variablenausprägungen die relationale Häufigkeit ihres gemeinsamen Auftretens dar. Variablenausprägungen, die weiter von einander entfernt liegen, kommen seltener (unterdurchschnittlich) gemeinsam vor als jene, die vergleichsweise näher (überdurchschnittlich) beieinander liegen. In der vorliegenden Arbeit beschränkt sich die Interpretation auf die Nähe und Distanz der im Forschungsinteresse stehenden Faktoren.

\section{(III) 3. Analysestrategie}

Die Beantwortung der Fragestellungen zur (1) sozialen Schließung und (2) Hierarchisierungen in der Freiwilligenarbeit sowie deren (3) feldspezifische Ausprägung erfolgt in vier Schritten.

In einem ersten Schritt (Kapitel (III).4.1.) wird die Bedeutung der einzelnen sozialen Merkmale (Geschlecht, Alter, Geburtsland, Staatsbürgerschaft, Bildungsgrad, Erwerbstätigkeit, Wohnrechtsverhältnis und Familienstand) für den Zugang zur Freiwilligenarbeit im Allgemeinen sowie zu den leitenden Positionen im Besondern bivariat analysiert.

Danach folgt in einem zweiten Schritt (Kapitel (III).4.2.) die multivariate Analyse (logistische Regressionsmodelle) der Determinanten des Zugangs zur Freiwilligenarbeit (soziale Schließung). Die Ergebnisse dazu werden mittels spezifischer Analysen weiter differenziert. Erstens, durch eine Gegenüberstellung 
der Determinanten des Zugangs zur Freiwilligenarbeit mit jenen des Zugangs zur Erwerbsarbeit. Zweitens, wird ausgehend von den Unterschieden in den Engagementmustern von Männern und Frauen der Einfluss der Determinanten getrennt nach dem Geschlecht analysiert und die Analyse um haushaltsbezogene Variablen (Haushaltsgröße sowie Anzahl und Alter der Kinder) ergänzt. Drittens wird ein Blick auf die Determinanten von Mehrfachengagement geworfen, ebenfalls getrennt nach Geschlecht. Viertens werden die für den Zugang der untersten Bildungsschicht relevanten Ressourcen analysiert. Abschließend erfolgt die Auswertung der subjektiv wahrgenommenen Barrieren des Zugangs zur Freiwilligenarbeit sowie deren Determinanten.

Im dritten Schritt (Kapitel (III).4.3.) werden die Determinanten der Hierarchisierung in der Freiwilligenarbeit, d.h. konkret des Zugangs zu leitenden Positionen in den Fokus der Analyse gestellt. Einer multivariaten Analyse zu den Einflussfaktoren folgen analog zu Schritt zwei, differenziertere Auswertungen. Wiederum erstens, Analysen zum Vergleich der Hierarchisierung in der Freiwilligenarbeit und der Erwerbsarbeit. Zweitens Auswertungen zum Einfluss von Mehrfachengagement auf die Position innerhalb der Freiwilligenarbeit. Drittens werden die Unterschiede zwischen Männern und Frauen analysiert und die Modelle um Variablen zum Berufsprestige erweitert. Den Abschluss dieses Kapitels bilden bivariate Analysen zum Zusammenhang von (Erwerbs-)Arbeitszeit und Freiwilligenarbeit.

Die feldspezifischen Analysen zum Zugang und zur Hierarchisierung in der Freiwilligenarbeit folgen im vierten und letzten Schritt (Kapitel (III).4.4.). Zur differenzierteren Analyse wurden vier, für die Freiwilligenarbeit zentrale Felder ausgewählt (Soziales und Gesundheit; Kirche und Religion; Sport und Bewegung; Katastrophenhilfs- und Rettungsdienste). Einleitend wird die Auswahl der vier Felder begründet und anschließend die Über- und Unterrepräsentanz der sozialen Merkmalsgruppen in diesen Feldern analysiert, grafisch dargestellt und sofern verfügbar mit empirischen Ergebnissen anderer Studien verglichen. Der bivariaten Gegenüberstellung folgen die multivarianten Analysen (logistische Regressionsmodelle) zu den feldspezifischen Determinanten des Zugangs. Abschließend werden die Regeln des Zugangs zu den unterschiedlichen hierarchischen Positionen in den vier Feldern mittels multiplen Korrespondenzanalysen untersucht. 


\section{(III) 4. Ergebnisse}

\section{(III) 4.1. Bivariate Analysen}

In einem ersten Schritt wird die Verteilung der askriptiven und erworbenen Merkmale (1) in der Österreichischen Bevölkerung (Grundgesamtheit) ${ }^{64}$, (2) der Respondentengruppe, (3) der Freiwilligen sowie (4) der leitenden Freiwilligen gegenübergestellt. Die Abbildungen (Scherengrafiken) auf den nachfolgenden Seiten zeigen die Struktur hinsichtlich der sozialen Merkmale in der Grundgesamtheit (kurz: $O ̈ \geq 15$ Jahre), der Respondentengruppe der Mikrozensus-Zusatzerhebung „Freiwilligenarbeit“ (kurz: MZ-Sample), der Gruppe der Freiwilligen (kurz: Freiwillige-gesamt) sowie der Gruppe der Freiwilligen deren Positionen mit Leitungsfunktionen verbunden sind (kurz: Freiwillige-leitend). Die Linien zwischen den Punkten stellen keine interpolierten Verläufe dar und sind somit auch nicht im statistischen Sinne zu interpretieren. Für die Darstellung in Form der „Scherengrafiken“ wurden jeweils auch mehrstufige und intervallskalierte Variablen (Alter, Bildungsgrad, Familienstand) dichotomisiert ${ }^{65}$.

\section{(III) 4.1.1. Geschlecht}

Der Anteil der Frauen in der Grundgesamtheit (Ö $\geq 15$ Jahre) von knapp über $52 \%$ bleibt im MZ-Sample konstant. In der Freiwilligenarbeit hingegen sinkt der Frauenanteil auf $44 \%$ und unter den Freiwilligen in Positionen mit Leitungsfunktion sinkt der Anteil weiter auf rund 31\% (siehe Abbildung 14). Vergleicht man die Geschlechterstruktur in der Freiwilligenarbeit mit jener in der Erwerbsarbeit, zeigen sich deutliche Parallelen. Der Anteil der Frauen an der erwerbstätigen Bevölkerung lag 2006 bei 45,3\% und der Anteil der Frauen unter den Erwerbstätigen mit Leitungsfunktion lag bei 31,4\% (vgl. Statistik Austria - Mikrozensus 2006/Q4).

64 Es werden hier die ungewichteten Daten des Mikrozensus herangezogen.

65 Die Tabellen zu den Häufigkeiten und Anteilen der vollständigen Variablenausprägungen finden sich weiter oben im Text im Rahmen der Samplebeschreibung sowie im Anhang. 
Abbildung 14: Geschlecht und Alter
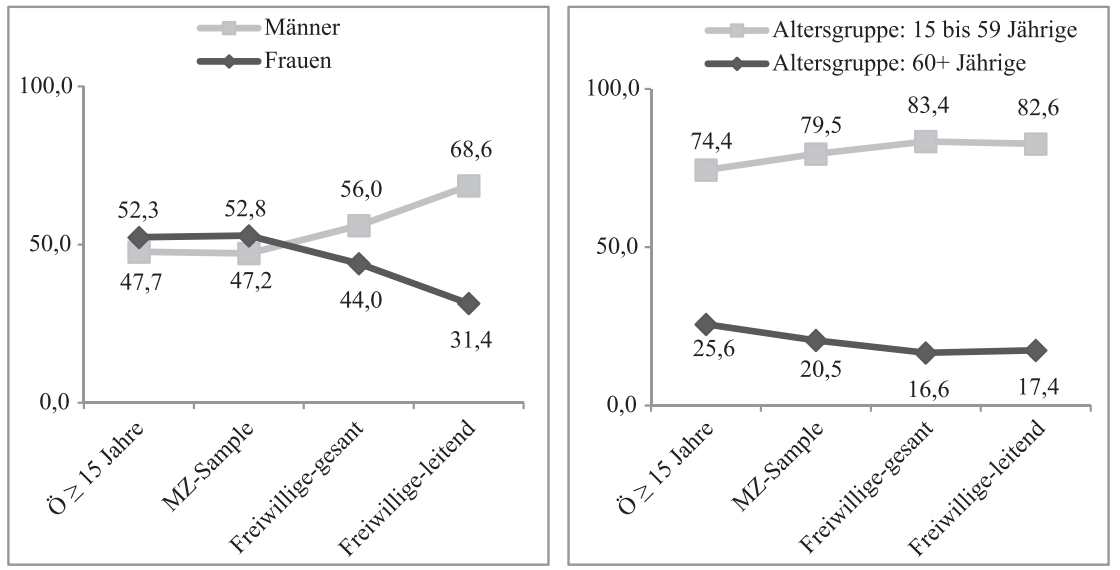

Quelle: Mikrozensus-Zusatzerhebung 2006/Q4; eigene Berechnungen; ungewichtet.

\section{(III) 4.1.2. Alter}

Aufgrund des Rückgangs der Beteiligungsquoten mit dem Pensionseintritt wurden für die bivariate Analyse die Befragten in die Gruppe der 15- bis 59-Jährigen und in die Gruppe der über 60-Jährigen aufgeteilt. Die Abbildung 14 zeigt, dass sich der Anteil der über 60-Jährigen von 25,6\% in der Grundgesamtheit auf 20,5\% im Sample der Freiwilligenerhebung reduziert. Unter den Freiwilligen selbst sind nur mehr rund $17 \%$ der Personen über 60 Jahre alt. Der Anteil von 17\% der über 60-Jährigen bleibt unter den Freiwilligen mit Leitungsfunktion konstant. Vergleicht man die Altersstruktur der Freiwilligenarbeit mit jener der Erwerbsarbeit, zeigt sich erwartungsgemäß ein deutlicher Unterschied. Dem Anteil von 17\% an über 60-Jährigen unter den Freiwilligen steht ein Anteil von rund 3\% dieser Gruppe an der erwerbstätigen Bevölkerung in Österreich gegenüber. Ab dem Pensionseintritt nehmen zwar auch die Beteiligungsquoten an der Freiwilligenarbeit mit zunehmenden Alter weiter ab (Backes/Höltge 2008; More-Hollerweger/Rameder 2009; Rameder/More-Hollerweger 2009), gesamt gesehen scheint Freiwilligenarbeit jedoch durchschnittlich für jeden Fünften über 60 Jahre ein Betätigungsfeld darzustellen ${ }^{66}$. Wie erwähnt, stellt Freiwilligenarbeit vor allem für jene Menschen im Ruhestand ein Handlungsfeld dar, die

66 Die Beteiligungsquote (gewichtet) der über 60-Jährigen liegt bei 19,3\% (Quelle: Mikrozensus-Zusatzerhebung 2006/Q4; eigene Berechnung) 
bereits früher im Lebenslauf Erfahrungen als Freiwillige gesammelt haben (vgl. Erlinghagen 2008; Erlinghagen et al. 2006).

\section{(III) 4.1.3. Ethnische Herkunft}

Die ethnische Herkunft (Geburtsland) betreffend zeigt sich, dass sich der Anteil von Personen, die nicht in Österreich geboren sind von rund 13\% in der Grundgesamtheit, bereits bei der Teilnahme an der Befragung zur Freiwilligenarbeit (MZ-Sample) auf $8 \%$ reduziert (siehe Abbildung 15). Der Anteil verringert sich unter den Freiwilligen weiter auf 5,4\%. Bei den Positionen mit Leitungsfunktion steigt der Anteil wiederum leicht um einen Prozentpunkt auf 6,4\% an. Im Vergleich liegt der Anteil von nicht in Österreich geborenen Personen unter der erwerbstätigen Bevölkerung bei 15,6\% und bei beruflichen Tätigkeiten mit Leitungsfunktionen bei 8,7\%. In der formellen Freiwilligenarbeit erscheint die ethnische Herkunft gemessen am Geburtsland ein „härteres“ Selektionskriterium zu sein als dies in der Erwerbsarbeit der Fall ist.

Abbildung 15: Geburtsland und Staatsbürgerschaft
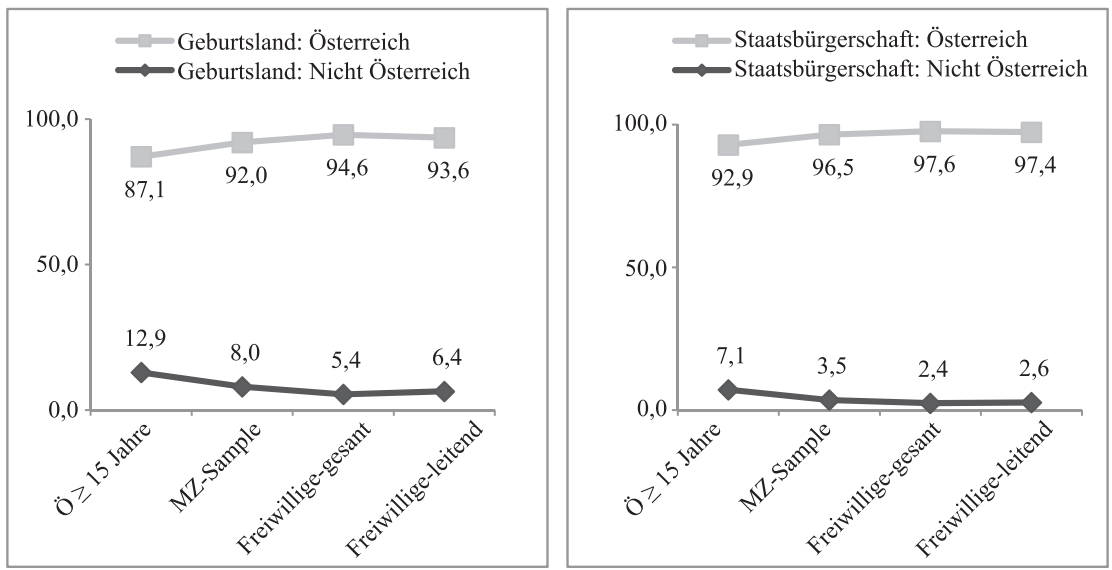

Quelle: Mikrozensus-Zusatzerhebung 2006/Q4; eigene Berechnungen; ungewichtet.

\section{(III) 4.1.4. Staatsbürgerschaft}

Personen ohne österreichische Staatsbürgerschaft sind in der Freiwilligenarbeit nur zu 2,4\% vertreten und das, obwohl ihr Anteil in der Bevölkerung bei 7,1\% liegt. Wie die wenigen Studien zu diesem Themengebiet bestätigen, haben in der Migrationspopulation informelle Formen der Freiwilligenarbeit eine größere Bedeutung 
als formelle Engagementformen (vgl. Reinprecht 2009). Darüber hinaus spiegelt sich in den vorliegenden Daten das bekannte Problem wider, dass mit den klassischen Instrumenten der Survey Forschung Personen aus der Migrationspopulation vergleichsweise schwer erreichbar sind (vgl. Meyer/Rameder 2011:18).

\section{(III) 4.1.5. Bildungsgrad}

Neben den zentralen askriptiven Merkmalen (Geschlecht, Alter, ethnische Herkunft) hat auch der sozio-ökonomische Status (Einkommen bzw. Vermögen, Bildungsgrad und beruflicher Status) einen bedeutsamen Einfluss auf den Zugang zur Freiwilligenarbeit. Der Bildungsgrad, verstanden als kulturelles Kapital und Ressource, ist dabei für Freiwilliges Engagement von zentraler Bedeutung. Entsprechend des vielfach bestätigten Zusammenhangs von Bildungsgrad und Freiwilligenarbeit sinkt der Anteil von Personen der bildungsfernsten Schicht, d.h. Person ohne Schulabschluss bzw. höchstens Pflichtschulabschluss, von 28,7\% (Ö $\geq 15$ Jahre) bereits auf 20\% bei der Erhebungsteilnahme (MZ-Sample). Unter den Freiwilligen sinkt der Anteil der untersten Bildungsschicht auf 15,0\%, und lediglich 6,9\% der leitenden Freiwilligen zählen zu dieser Gruppe. Vom Kompetenzerwerb durch Freiwilligenarbeit bleiben damit gerade die bildungsfernsten Schichten in einem hohen Maße ausgeschlossen. In welcher Weise der Einfluss der Bildung auf Freiwilliges Engagement durch Merkmale wie Alter, Geschlecht und ethnische Herkunft (vgl. Musick/Wilson 2008: 124ff) auch in Österreich moderiert wird, werden die multivariaten Analysen in den nachfolgenden Kapiteln zeigen.

Abbildung 16: Bildungsgrad und Erwerbstätigkeit
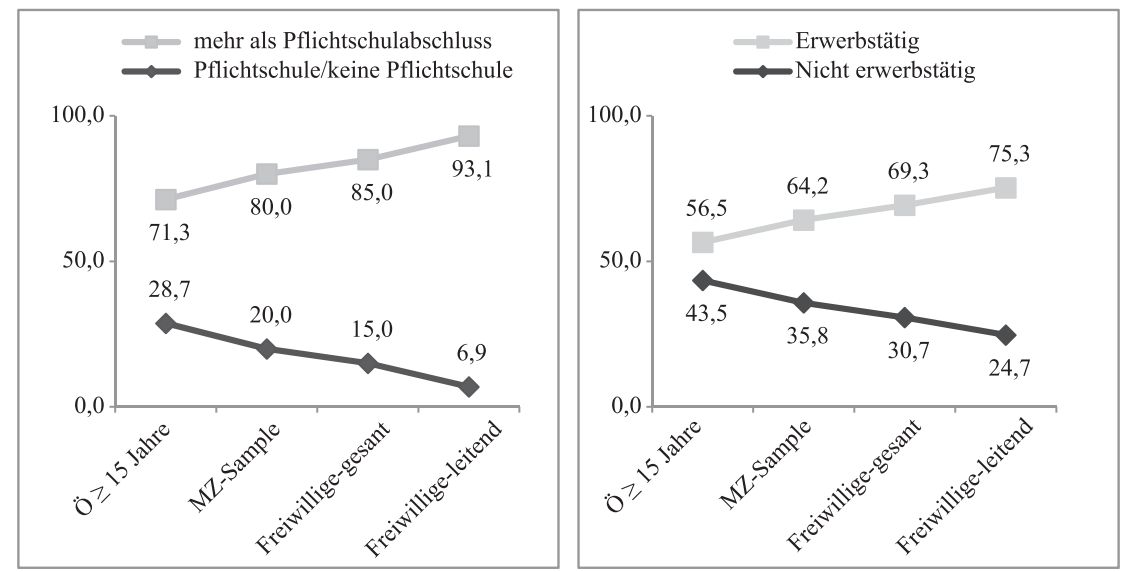

Quelle: Mikrozensus-Zusatzerhebung 2006/Q4; eigene Berechnungen; ungewichtet. 


\section{(III) 4.1.6. Erwerbstätigkeit}

Ein zweiter Indikator für den sozio-ökonomischen Status ist die Erwerbstätigkeit im Allgemeinen wie der konkrete Berufsstatus im Besonderen. Der Anteil von Personen die einer beruflichen Tätigkeit nachgehen, erhöht sich von 56,5\% in der Grundgesamtheit auf 64,2\% im Mikrozensus Sample und weiter auf 69,3\% bei den Freiwilligen. Bei den Freiwilligen in Positionen mit Leitungsfunktion sind 75,3\% erwerbstätig. Umgekehrt betrachtet halbiert sich der Anteil der Nicht-Erwerbstätigen (Arbeitslose, Pensionistinnen und Pensionisten und haushaltsführende Personen) von 43,5\% in der Grundgesamtheit (> 15 Jahren) auf rund 24,7\% unter den leitenden Freiwilligen. Hier gilt es im besonderen Maße den moderierenden Einfluss von Alter und Geschlecht in den multivariaten Analysen zu beachten.

\section{(III) 4.1.7. Wohnrechtsverhältnis}

Das Einkommen und das Vermögen bilden die dritte Säule des sozio-ökomischen Status. Wie in der Beschreibung des Datensatzes festgestellt, werden im Rahmen des Mikrozensus keine Daten zum Einkommen und Vermögen der Befragten erhoben. Aus diesem Grund kann der Zusammenhang zwischen Freiwilligenarbeit, Einkommen und Vermögen lediglich über die Variable des sogenannten „Wohnrechtsverhältnisses" und in weiterer Folge über den Berufsstatus approximiert werden.

In Österreich leben rund 41\% (der über 14-Jährigen) in Miete und rund 59\% in Wohneigentum, d.h. in Wohnungs- oder Hauseigentum ${ }^{67}$. Diese Verteilung verschiebt sich unter den zur Freiwilligenarbeit Befragten auf knapp 70\% in Wohneigentum Lebenden. Unter den Freiwilligen selbst erhöht sich der Anteil nochmals auf 77\%. Unter den Freiwilligen mit Leitungsfunktion verändert sich der Anteil mit 78,6\% kaum mehr. Damit dürfte, wie in anderen Studien bereits bestätigt, eine bestimmte finanzielle Grundabsicherung für den Zugang zur Freiwilligenarbeit nötig sein (vgl. Musick/Wilson 2008: 127ff) bzw. Wohneigentum zu einer verstärkten sozialen Verwurzelung und damit verbunden einem größeren Interesse am Gemeinwesen führen (vgl. Rotolo et al. 2010). Auf die interne, hierarchische Strukturierung scheint das Wohnrechtsverhältnis zumindest bivariat keinen bedeutsamen Einfluss mehr haben.

67 Die Variable enthält keine Informationen über den monetären Wert der Immobilien bzw. über deren etwaige Fremdfinanzierung. 
Abbildung 17: Wohnrechtsverhältnis und Familienstand
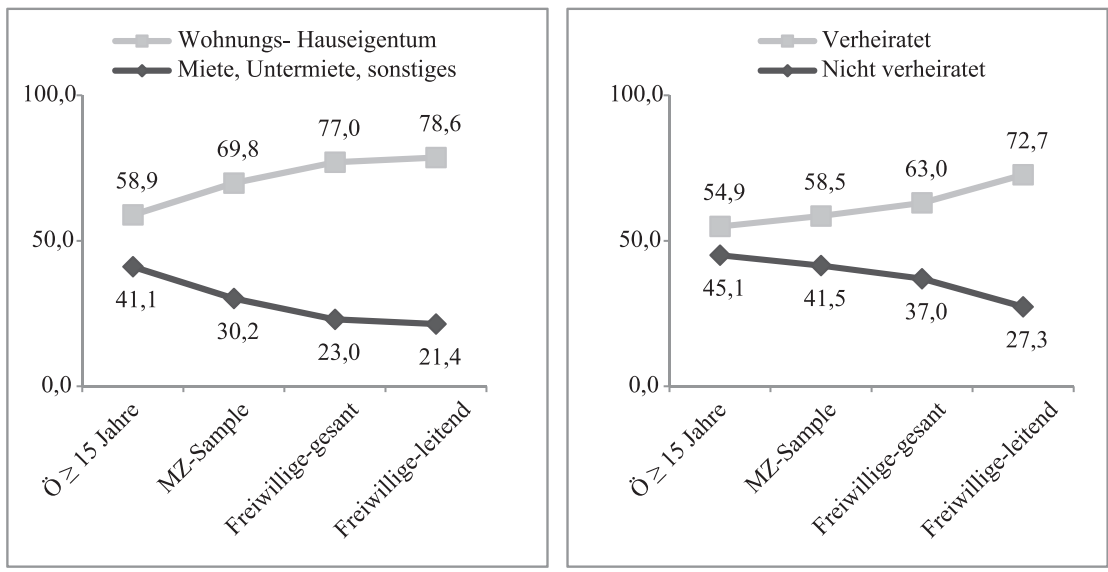

Quelle: Mikrozensus-Zusatzerhebung 2006/Q4; eigene Berechnungen; ungewichtet.

\section{(III) 4.1.8. Familienstand}

Der Familienstand, in diesem Fall reduziert auf die traditionelle Dichotomie verheiratet und nicht verheiratet (ledig, geschieden, verwitwet), weist ebenfalls einen Zusammenhang mit Freiwilligem Engagement auf. So erhöht sich der Anteil der Verheirateten von rund 55\% in der Grundgesamtheit auf $63 \%$ bei den Freiwilligen und nochmals auf $72,7 \%$ bei Freiwilligen mit Leitungsfunktion. Ob hinter dem Familienstand der wiederholt bestätigte positive Einfluss der Religiosität steht (vgl. Taniguchi/Thomas 2011; van Tienen et al. 2011; Vermeer/ Scheepers 2012), kann mangels entsprechender Variablen zur Konfession bzw. zur tatsächlichen Religionsausübung nicht beantwortet werden. Weiterführende Analysen zu den Interaktionseffekten des Familienstands mit dem Geschlecht könnten jedoch Hinweise liefern, ob hinter dem Familienstand eher religiöse Einstellungen oder primär das Sozialsystem der Familie bzw. vielfach weiterhin wirksamen Geschlechterrollenstereotypen stehen.

\section{(III) 4.1.9. Zusammenfassung der Ergebnisse}

In der Zusammenschau zeigt der bivariate Vergleich der Sozialstruktur der österreichischen Bevölkerung mit (1) den Befragten im Mikrozensusdatensatzes zur Freiwilligenarbeit, (2) den Freiwilligen sowie (3) den Freiwilligen mit Leitungsfunktion eine Unterrepräsentanz bei Personen, die folgenden sozialen Merkmalsgruppen zugehören: 
Tabelle 12: Übersicht zu den bivariaten Ergebnissen zur sozialen Schließung

\begin{tabular}{|c|c|c|c|}
\hline & \multicolumn{3}{|c|}{ Soziale Schließung bezüglich... } \\
\hline & $\begin{array}{c}\text { (1) der } \\
\text { Teilnahme an } \\
\text { der Befragung } \\
\text { (MZ-Sample) }\end{array}$ & $\begin{array}{l}\text { (2) des Zugangs zu } \\
\text { Freiwilligenarbeit } \\
\text { (Freiwillige-gesamt) }\end{array}$ & $\begin{array}{l}\text { (3) des Zugangs zu } \\
\text { Leitungspositionen } \\
\text { (Freiwillige-leitend) }\end{array}$ \\
\hline Geschlecht: Frau & & $\mathrm{x}$ & $\mathrm{x}$ \\
\hline Alter: $60+$ & $\mathrm{x}$ & $\mathrm{x}$ & (x) \\
\hline $\begin{array}{l}\text { Geburtsland: } \\
\text { Nicht-Österreich }\end{array}$ & $\mathrm{x}$ & $\mathrm{x}$ & (x) \\
\hline $\begin{array}{l}\text { Staatsbürgerschaft: } \\
\text { Nicht-Österreich }\end{array}$ & $\mathrm{x}$ & $\mathrm{x}$ & (x) \\
\hline $\begin{array}{l}\text { Bildungsgrad: } \\
\text { Pflichtschulabschluss }\end{array}$ & $\mathrm{x}$ & $\mathrm{x}$ & $\mathrm{x}$ \\
\hline Nicht-Erwerbstätig & $\mathrm{x}$ & $\mathrm{x}$ & $\mathrm{x}$ \\
\hline $\begin{array}{l}\text { Wohnrechtsverhältnis: } \\
\text { Miete }\end{array}$ & $\mathrm{x}$ & $\mathrm{x}$ & (x) \\
\hline $\begin{array}{l}\text { Familienstand: Nicht } \\
\text { verheiratet }\end{array}$ & $\mathrm{x}$ & $\mathrm{x}$ & $\mathrm{x}$ \\
\hline
\end{tabular}

1. Schon bei der (freiwilligen) Teilnahme an der Mikrozensus-Zusatzbefragung zur Freiwilligenarbeit zeigen sich erste Schließungstendenzen. Personen über 60 Jahren, Migrantinnen und Migranten (Geburtsland sowie Staatsbürgerschaft), untere Bildungsschichten, Nicht-Erwerbstätige, in Miete lebende und Nicht-Verheiratete haben überdurchschnittlich oft eine Teilnahme an der Befragung abgelehnt. Lediglich hinsichtlich des Geschlechts entspricht das Sample der Verteilung in der Gesamtbevölkerung.

2. Bei der Freiwilligenarbeit selbst sind Frauen unterrepräsentiert und alle weiteren, schon bei der Befragung bedeutsamen sozialen Merkmale bleiben ebenso schließungsrelevant: Alter, Geburtsland, Staatsbürgerschaft, Bildungsgrad, Erwerbstätigkeit, Wohnrechtsverhältnis und Familienstand.

3. Innerhalb der Freiwilligenarbeit fungieren weiterführend primär das Geschlecht, der Bildungsgrad, der Erwerbsstatus und der Familienstand als Determinanten der internen hierarchischen Strukturierung. Frauen, Angehörige der untersten Bildungsschicht, in Miete lebende sowie unverheiratete Personen sind innerhalb der Freiwilligenarbeit überdurchschnittlich vom $\mathrm{Zu}$ gang zu leitenden Positionen ausgeschlossenen. Geht man nicht von bereits 
freiwillig Engagierten aus, sondern von der österreichischen Gesamtbevölkerung, sind alle ausgewählten Merkmale für den Zugang zu hierarchischen Positionen in der Freiwilligenarbeit bedeutsam.

Somit wurde in einem ersten Schritt anhand der bivariaten Analysen sichtbar welche sozialen Merkmale für den Zugang zur Freiwilligenarbeit in Österreich von Bedeutung sind und welche Bevölkerungsgruppen tendenziell von sozialen Schließungsprozessen in der Freiwilligenarbeit betroffen sind. Genaueren Aufschluss darüber liefern jedoch nur multivariate Analysen. Diese folgen in den nächsten drei Kapiteln.

\section{(III) 4.2. Multivariate Analysen zur sozialen Schließung in der Freiwilligenarbeit}

Die zentrale Frage in diesem Kapital lautet: Welchen Einfluss haben askriptive wie erworbene soziale Merkmale (Geschlecht, Alter, ethnische Herkunft, Bildungsgrad, Vermögen, berufliche Stellung und Prestige) auf den Zugang zur Freiwilligenarbeit in Österreich? Ergänzend werden Analysen zu Fragen durchgeführt, die sich erst im Zuge der Beantwortung der obigen Ausgangsfrage gestellt haben. Diese umfassen den Vergleich der Determinanten des Zugangs zur Freiwilligenarbeit mit jenen des Zugangs zur Erwerbsarbeit; die Gegenüberstellung der Determinanten des Engagements von Männern und Frauen; die Analyse der Determinanten von Mehrfachengagement; Detailanalysen zum Zugang der untersten Bildungsschicht und abschließend Auswertungen zu den subjektiven Barrieren des Zugangs zur Freiwilligenarbeit und deren Determinanten.

\section{(III) 4.2.1. Determinanten des Zugangs zur Freiwilligenarbeit}

Wie aus der logistischen Regression in Tabelle 13 ersichtlich wird, werden bis auf den Einfluss der Erwerbstätigkeit alle, oben in den Scherengrafiken gezeigten Einflüsse auch in der multivariaten Analyse in ihrer Wirkrichtung bestätigt. Damit decken sich die Ergebnisse zu den Determinanten der formellen Freiwilligenarbeit, mit Ausnahme der Erwerbsarbeit, in ihrer Grundtendenz mit den Ergebnissen der europäischen wie internationalen Forschung. Dies deutet darauf hin, dass auch die nachfolgenden Ergebnisse zumindest in Ansätzen auf andere, mit der österreichischen Freiwilligenstruktur vergleichbare Länder wie z.B. Deutschland und die Schweiz übertragbar sein dürften. 
Tabelle 13: Logistische Regression - Einflussfaktoren auf den Zugang zur formellen Freiwilligenarbeit

\begin{tabular}{|c|c|c|c|c|c|c|}
\hline & \multicolumn{6}{|c|}{ Abhängige Variable: Formelle Freiwilligenarbeit } \\
\hline & \multicolumn{3}{|c|}{ Modell I } & \multicolumn{3}{|c|}{ Modell II } \\
\hline & Beta & SE & $\overline{\operatorname{Exp}(B)}$ & Beta & SE & $\operatorname{Exp}(B)$ \\
\hline Frauen (Ref. Männer) & $-0,581$ & 0,039 & $0,559^{* * *}$ & $-0,562$ & 0,04 & $0,570^{* * *}$ \\
\hline Alter & $-0,01$ & 0,001 & $0,990^{* * *}$ & $-0,01$ & 0,001 & $0,990^{* * *}$ \\
\hline Geburtsland (Ref. Österreich) & $-0,551$ & 0,08 & $0,576^{* * *}$ & $-0,543$ & 0,08 & $0,581^{* * *}$ \\
\hline Bildungsgrad ( 1 bis 6 ) & 0,200 & 0,014 & $1,222^{* * *}$ & 0,196 & 0,014 & $1,217^{* * *}$ \\
\hline $\begin{array}{l}\text { Erwerbstätig (Ref. Erwerbslos, } \\
\text { Arbeitslos, in Pension, Präsenz- } \\
\text { oder Zivildiener) }\end{array}$ & 0,049 & 0,046 & 1,050 & 0,01 & 0,049 & 1,010 \\
\hline $\begin{array}{l}\text { Leitenden Funktion im Beruf } \\
\text { (Ref. keine leitenden Funktion) }\end{array}$ & & & & 0,135 & 0,054 & $1,145^{* *}$ \\
\hline $\begin{array}{l}\text { Wohnrechtsverhältnis Eigentum } \\
\text { (Ref. Miete, Untermiete, } \\
\text { sonstiges) }\end{array}$ & 0,352 & 0,047 & $1,422^{* * *}$ & 0,351 & 0,047 & $1,421^{* * *}$ \\
\hline $\begin{array}{l}\text { Verheiratet (Ref. Ledig, } \\
\text { Geschieden, Verwitwet) }\end{array}$ & 0,334 & 0,045 & $1,396^{* * *}$ & 0,33 & 0,045 & $1,390^{* * *}$ \\
\hline Bevölkerungsdichte & $-0,301$ & 0,025 & $0,740^{* * *}$ & $-0,301$ & 0,026 & $0,740^{* * *}$ \\
\hline Konstante & 1,081 & 0,145 & $2,947^{* * *}$ & 1,068 & 0,145 & $2,911^{* * *}$ \\
\hline $\mathrm{N}$ & & 11.657 & & & 11.657 & \\
\hline $\mathrm{Chi}^{2} / \mathrm{df}$ & & $931 / 8$ & & & $936 / 9$ & \\
\hline -2 Log-Likelihood & & $14.874,4$ & & & $14.868,17$ & \\
\hline Pseudo R ${ }^{2}$ (Nagelkerke’s) & & 0,103 & & & 0,104 & \\
\hline
\end{tabular}

Quelle: Mikrozensus-Zusatzerhebung 2006/Q4; eigene Berechnung; $\left(p^{*}<0.05 ; p^{* *}<0.01 ; p^{* * *}<0.001\right)$.

Die Ergebnisse im Detail: Einen zentralen Einfluss auf den Zugang zur Freiwilligenarbeit hat das Geschlecht. So reduziert sich bei Frauen gegenüber Männern die Chance, sich in der Freiwilligenarbeit zu engagieren, um 44,1\% (odds ratio: $\left.0,559^{* * *}\right)$. Ähnliches gilt für die ethnische Herkunft. Nicht in Österreich geboren zu sein verringert die Wahrscheinlichkeit, Zugang zur formellen Freiwilligenarbeit zu finden, um 42,4\% (odds ratio: $0,576^{* * *}$ ) gegenüber gebürtigen Österreicherinnen und Österreichern. Die Höhe des Bildungsgrads hat erwartungsgemäß einen positiven Einfluss auf den Zugang zu Freiwilligenarbeit und 
bleibt trotz der Kontrolle der anderen Einflussfaktoren wie Geschlecht, Alter und ethnische Herkunft signifikant: Die Wahrscheinlichkeit für Freiwilliges Engagement erhöht sich je Bildungsgrad ${ }^{68}$ durchschnittlich um jeweils 22,2\% (odds ratio: $1,222^{* * *}$ ). Ob man erwerbstätig ist oder nicht, hat im Kanon der anderen Variablen mit einer odds ratio von 1,050 keinen signifikanten Einfluss auf den Zugang zur Freiwilligenarbeit. In diesem Punkt weichen die Ergebnisse aus Österreich teilweise von den Ergebnissen bestehender Studien (vgl. Musick/Wilson 2008: 148ff) ab. Es bleibt offen, ob dies dem Erhebungsdesign und der damit verbundenen Verzerrung (vgl. Abbildung 16) bei der Erhebungsteilnahme geschuldet ist, oder ob die Erwerbstätigkeit ${ }^{69}$ tatsächlich in Österreich eine, verglichen z.B. mit den USA, untergeordnete Rolle spielt. Aus der Regressionsanalyse in Modell II in Tabelle 13 wird ersichtlich, dass sich bei jenen Personen, die in ihrer beruflichen Tätigkeit eine leitenden Funktion innehaben die Chance, freiwillig engagiert zu sein, geringfügig um 14,5\% (odds ratio: $1,145^{* *}$ ) erhöht. Der Erklärungsbeitrag (Pseudo $\mathrm{R}^{2}$ ) des Modells erhöht sich dabei jedoch nur sehr gering von 0,103 in Modell I auf 0,104 in Modell II. Die Variablen zur Erwerbstätigkeit und der Funktion im Beruf leisten damit nur einen sehr kleinen Beitrag zur Erklärung der Unterschiede im Zugang zur Freiwilligenarbeit. Der verwendete Indikator für ökonomisches Kapital, das Wohnrechtsverhältnis, bestätigt mit einer odds ratio von $1,422^{* * *}$ den positiven Einfluss dieses Merkmals. In Wohneigentum zu leben erhöht die Wahrscheinlichkeit für formelles Engagement demnach um 42,2\%. Dieser Einfluss lässt sich vor dem Hintergrund der Forschung zweifach interpretieren. Das Wohnrechtsverhältnis, als Indikator für ein Mindestmaß an ökonomischem Kapital, unterstreicht die These, dass man sich Freiwilliges Engagement leisten können muss. Der Erklärungsansatz von Rotolo et al. (2010) knüpft zwar grundlegend auch an der Vermögensausstattung an, erklärt die höhere Engagementquote von Personen, die in Wohneigentum leben, jedoch durch deren dauerhafteren und damit verwurzelteren Bezug zum Wohnumfeld (stake in society) und den damit verbundenen größeren Interesse, dieses mitzugestalten und darin aktiv zu werden. Um diese beiden Effekte trennen zu können, müsste die Wohndauer mitanalysiert werden. Diese ist jedoch durch den Mikrozensus nicht erfasst. Einen ähnlich hohen positiven Einfluss hat jedoch auch der Familienstand. Verheiratet zu sein erhöht die Wahrscheinlichkeit,

68 6-stufige Skalierung (siehe Variablenbeschreibung).

69 Ersetz man die Variable „erwerbstätig“ durch die differenzierte Variable der beruflichen Stellung (siehe Variablenbeschreibung) erhöht dies die Erklärungskraft (Pseudo $\mathrm{R}^{2}$ (Nagelkerke's)) des Modeln lediglich von 0,104 auf 0,107. 
sich in der Freiwilligenarbeit zu engagieren um 39,6\% (odds ratio: 1,396 ${ }^{* *}$ ). Da auch die Wohnortgröße bzw. der Urbanisierungsgrad die Freiwilligenarbeit beeinflusst, wird den multivariaten Modellen die Bevölkerungsdichte als Kontrollvariable hinzugefügt. In beiden Modellen in Tabelle 13 wirkt sich eine höhere Bevölkerungsdichte negativ auf die Wahrscheinlichkeit (odds ratio: $\left.0,740^{* * *}\right)$ aus, Freiwilligenarbeit zu leisten.

Bis auf den Einfluss der Erwerbstätigkeit bestätigt die multivariate Analyse zur sozialen Schließung die bivariaten Ergebnisse zum Einfluss von Geschlecht, Alter, ethnischer Herkunft, Bildungsgrad (kulturelles Kapital), Wohnrechtsverhältnis (ökonomisches Kapital) und dem Familienstand. Die Erwerbstätigkeit im Allgemeinen hat unter Beachtung der anderen Faktoren keinen Einfluss auf den Zugang zur Freiwilligenarbeit, eine leitende Position im Beruf hingegen wirkt förderlich. Im Hinblick auf die in der Problemstellung kritisierte Überschätzung des integrativen Potentials der Freiwilligenarbeit macht auch die multivariate Überprüfung der Einflussfaktoren deutlich, dass die Freiwilligenarbeit in ihrer jetzigen Form betreffend der Inklusion von Frauen, Migrantinnen und Migranten und bildungsfernen Schichten kein Selbstläufer ist. Der große Einfluss des Wohnrechtsverhältnisses auf den Zugang zur Freiwilligenarbeit lässt ungeachtet der dahinterstehenden Mechanismen, die Frage aufkommen, wie sich die derzeitige Preisentwicklung am Wohnungsmarkt und das weitere Auseinanderklaffen der Einkommensschere auf die künftige Beteiligung an der Freiwilligenarbeit auswirken wird. Eine Zunahme der sozialen Ungleichheit in den Dimensionen Einkommen und Vermögen sowie betreffend die Wohnverhältnisse beeinflusst demzufolge auch die Prozesse sozialer Schließung in der Freiwilligenarbeit.

Ausgehend von den Ergebnissen zum Einfluss des Geschlechts, Alters, der ethnischer Herkunft und des Bildungsgrads lässt sich die These formulieren, dass Freiwilligenarbeit und Erwerbsarbeit in weiten Bereichen ähnliche $\mathrm{Zu}$ gangsvoraussetzungen aufweisen.

\section{(III) 4.2.2. Determinanten des Zugangs zur Freiwilligenarbeit und Erwerbsarbeit im Vergleich}

Vergleicht man den Einfluss der askriptiven wie erworbenen Merkmale für den Zugang zur Freiwilligenarbeit mit deren Bedeutung für den Zugang zur Erwerbsarbeit, zeigt sich folgendes Bild: Frauen haben in beiden Feldern eine um rund $45 \%$ geringere Wahrscheinlichkeit tätig zu sein als Männer. Nicht in Österreich geboren zu sein wirkt sich sowohl in der Erwerbsarbeit als auch der Freiwilligenarbeit negativ auf die Zugangschancen aus (odds ratio: 0,738 
und $0,574^{* * *}$ ). In beiden Feldern hat der Bildungsgrad einen signifikant positiven Einfluss. In Eigentum zu leben ist hingegen nur für die Freiwilligenarbeit von signifikanter Bedeutung (odds ratio: $1,422^{* * *}$ ). Verheiratet $\mathrm{zu}$ sein ist ebenfalls in der Erwerbsarbeit wie in der Freiwilligenarbeit von großem Einfluss (odds ratio: $2,308^{* * *}$ und $1,407^{* * *}$ ). Die verwendeten unabhängigen Variablen erklären dabei jedoch den Zugang zur Erwerbsarbeit mit einem Pseudo $\mathrm{R}^{2}$ von 0,343 besser als den Zugang zur Freiwilligenarbeit mit einem Pseudo $\mathrm{R}^{2}$ von 0,103 .

Tabelle 14: Logistische Regression - Determinanten des Zugangs zur Erwerbsarbeit und der Freiwilligenarbeit

\begin{tabular}{|c|c|c|c|c|c|c|}
\hline & \multicolumn{3}{|c|}{$\begin{array}{l}\text { Abhängige Variable: } \\
\text { Erwerbsarbeit }\end{array}$} & \multicolumn{3}{|c|}{$\begin{array}{c}\text { Abhängige Variable: } \\
\text { Formelle } \\
\text { Freiwilligenarbeit }\end{array}$} \\
\hline & Beta & SE & $\operatorname{Exp}(B)$ & Beta & SE & $\operatorname{Exp}(B)$ \\
\hline Frauen (Ref. Männer) & $-0,605$ & 0,023 & $0,546^{* * *}$ & $-0,586$ & 0,039 & $0,557^{* * *}$ \\
\hline Alter & $-0,063$ & 0,001 & $0,939^{* * *}$ & $-0,011$ & 0,001 & $0,990^{* * *}$ \\
\hline Geburtsland (Ref. Österreich) & $-0,304$ & 0,036 & $0,738^{* * *}$ & $-0,555$ & 0,080 & $0,574^{* * *}$ \\
\hline Bildungsgrad & 0,357 & 0,009 & $1,428^{* * *}$ & 0,203 & 0,014 & $1,225^{* * *}$ \\
\hline $\begin{array}{l}\text { Wohnrechtsverhältnis Eigentum } \\
\text { (Ref. Miete, Untermiete, sonstiges) }\end{array}$ & $-0,009$ & 0,027 & 0,991 & 0,352 & 0,047 & $1,422^{* * *}$ \\
\hline $\begin{array}{l}\text { Verheiratet (Ref. Ledig, } \\
\text { Geschieden, Verwitwet) }\end{array}$ & 0,836 & 0,027 & $2,308^{* * *}$ & 0,342 & 0,044 & $1,407^{* * *}$ \\
\hline Bevölkerungsdichte & $-0,081$ & 0,015 & $0,923^{* * *}$ & $-0,302$ & 0,025 & $0,739^{* * *}$ \\
\hline Konstante & 3,299 & 0,076 & $27,090^{* * *}$ & 1,141 & 0,133 & $3,130^{* * *}$ \\
\hline $\mathrm{N}$ & \multicolumn{3}{|c|}{41.412} & \multicolumn{3}{|c|}{11.657} \\
\hline $\mathrm{Chi}^{2} / \mathrm{df}$ & \multicolumn{3}{|c|}{$12238 / 7$} & \multicolumn{3}{|c|}{$930 / 7$} \\
\hline -2 Log-Likelihood & \multicolumn{3}{|c|}{44458,712} & \multicolumn{3}{|c|}{14875,52} \\
\hline Pseudo R² (Nagelkerke’s) & \multicolumn{3}{|c|}{0,343} & \multicolumn{3}{|c|}{0,103} \\
\hline
\end{tabular}

Quelle: Mikrozensus-Zusatzerhebung 2006/Q4; eigene Berechnung; $\left(p^{*}<0.05 ; p^{* *}<0.01\right.$; $\left.p^{* * *}<0.001\right)$.

Somit decken sich in der Erwerbsarbeit und der Freiwilligenarbeit, bis auf die Variable zur Vermögensausstattung (Wohnrechtsverhältnis), die Wirkrichtung und die Signifikanz der Determinanten. Daraus lässt sich zumindest ansatzweise schließen, dass die formelle Freiwilligenarbeit nicht frei von organisationalen 
Zugangs- und Selektionsmechanismen ist, wie sie in den Feldern der Erwerbsarbeit üblich sind. Zusätzlich ist für den Zugang zur Freiwilligenarbeit auch noch ein Mindestmaß an ökonomischem Kapital nötig. Vor dem Hintergrund dieser Ergebnisse scheint das Integrationspotential der Freiwilligenarbeit nicht größer zu sein als jenes der Erwerbsarbeit. Damit bestätigt sich auch die These, dass gesellschaftliche Integration primär über die Integration in die (Erwerbs-) Arbeit erfolgt und erfolgen sollte, und Freiwilligenarbeit derzeit vielmehr Ausdruck gelungener Integration als Mittel zur Integration ist. Damit stellt sich auch die Frage, ob die Bereiche bzw. die Felder der Freiwilligenarbeit tatsächlich als eigene Kontextebene der Genese sozialer Ungleichheit zu denken sind, oder aber diese, wie es Diewald/Faist (2010) implizit vorgeschlagen haben, gemeinsam mit der Erwerbsarbeit in die Analyse der ungleichheits(re)produzierenden Wirkungen und Prozesse jeglicher Ausprägungsformen formaler Organisationen miteinzuschließen sind.

Als kleiner Exkurs sei noch die Gegenprobe erwähnt: Tauscht man in der Regressionsanalyse die „Freiwilligenarbeit“ mit der „Erwerbsarbeit“, d.h. macht die Erwerbsarbeit zur abhängigen Variable und fügt die Freiwilligenarbeit als erklärende Variable dem Modell hinzu (siehe Tabelle 47 im Anhang), zeigt sich, dass die Freiwilligenarbeit keinen signifikanten Einfluss auf den Zugang zur Erwerbsarbeit hat ${ }^{70}$. Durch das Hinzufügen der Freiwilligenarbeit als erklärende Variable erhöht sich das Pseudo $\mathrm{R}^{2}$ lediglich um 0,001 von 0,287 in Model I auf Model II 0,288.

\section{(III) 4.2.3. Determinanten des Zugangs von Frauen und Männern im Vergleich}

Die bisherige Forschung hat wiederholt gezeigt, dass vor allem sozioökonomische Faktoren (z.B. Berufstätigkeit, Teilzeit/Vollzeit) bei Frauen und Männern einen unterschiedlichen Einfluss auf den Zugang zur Freiwilligenarbeit haben (vgl. Marshall/Taniguchi 2012; Musick/Wilson 2008).

70 Die Samplegröße im Regressionsmodell zur Erklärung der Erwerbsarbeit reduziert sich durch die Hinzunahme der Freiwilligenarbeit als erklärende Variable von ursprünglich $\mathrm{N}=20.104$ auf den $\mathrm{N}=11.657$. 
Tabelle 15: Logistische Regression - Formelle Freiwilligenarbeit und der Einfluss der Leitungsfunktion im Beruf getrennt nach Frauen und Männer

\begin{tabular}{|c|c|c|c|c|c|c|}
\hline & \multicolumn{6}{|c|}{ Abhängige Variable: Formelle Freiwilligenarbeit } \\
\hline & \multicolumn{3}{|c|}{ Frauen } & \multicolumn{3}{|c|}{ Männer } \\
\hline & Beta & SE & $\overline{\operatorname{Exp}(B)}$ & Beta & SE & $\operatorname{Exp}(B)$ \\
\hline Alter & $-0,009$ & 0,002 & $0,991^{* * *}$ & $-0,010$ & 0,002 & $0,990^{* * *}$ \\
\hline Geburtsland (Ref. Österreich) & $-0,466$ & 0,108 & $0,628^{* * *}$ & $-0,646$ & 0,118 & $0,524^{* * *}$ \\
\hline Bildungsgrad & 0,233 & 0,020 & $1,262^{* * *}$ & 0,163 & 0,020 & $1,177^{* * *}$ \\
\hline $\begin{array}{l}\text { Erwerbstätig (Ref. Erwerbslos, } \\
\text { Arbeitslos, in Pension, Präsenz- oder } \\
\text { Zivildiener) }\end{array}$ & $-0,015$ & 0,065 & 0,985 & 0,030 & 0,074 & 1,031 \\
\hline $\begin{array}{l}\text { Leitenden Funktion im Beruf } \\
\text { (Ref. keine leitenden Funktion) }\end{array}$ & 0,052 & 0,091 & 1,053 & 0,191 & 0,069 & $1,210^{* *}$ \\
\hline $\begin{array}{l}\text { Wohnrechtsverhältnis Eigentum } \\
\text { (Ref. Miete, Untermiete, sonstiges) }\end{array}$ & 0,332 & 0,067 & $1,394^{* * *}$ & 0,357 & 0,066 & $1,429^{* * *}$ \\
\hline $\begin{array}{l}\text { Verheiratet (Ref. Ledig, Geschieden, } \\
\text { Verwitwet) }\end{array}$ & 0,386 & 0,060 & $1,472^{* * *}$ & 0,272 & 0,068 & $1,312^{* * *}$ \\
\hline Bevölkerungsdichte & $-0,214$ & 0,036 & $0,807^{* * *}$ & $-0,390$ & 0,036 & $0,677^{* * *}$ \\
\hline Konstante & $-0,434$ & 0,179 & $0,648^{*}$ & 0,857 & 0,191 & $2,355^{* * *}$ \\
\hline $\mathrm{N}$ & & 6155 & & & 5502 & \\
\hline $\mathrm{Chi}^{2} / \mathrm{df}$ & & $344 / 8$ & & & $368 / 8$ & \\
\hline -2 Log-Likelihood & & 7579,061 & & & 7257,825 & \\
\hline Pseudo R² (Nagelkerke’s) & & 0,075 & & & 0,086 & \\
\hline
\end{tabular}

Quelle: Mikrozensus-Zusatzerhebung 2006/Q4; eigene Berechnung; $\left(p^{*}<0.05 ; p^{* *}<0.01\right.$; $\left.p^{* * *}<0.001\right)$.

Bei einem ersten Blick auf die Ergebnisse in Tabelle 15 zeigt sich, dass in Bezug auf die analysierten Einflussfaktoren keine grundlegenden Unterschiede zwischen Männern und Frauen bestehen. Der Einfluss des Alters, der ethnischen Herkunft, des Bildungsgrades, der Erwerbstätigkeit, des Wohneigentums, des Familienstandes wie der Bevölkerungsdichte deckt sich grundsätzlich in Wirkrichtung und Signifikanz. Genauer betrachtet zeigt sich, dass für Frauen

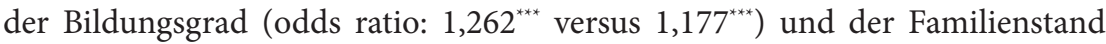
(odds ratio: $1,394^{* * *}$ versus 1,429**) mehr Bedeutung für den Zugang zur Freiwilligenarbeit hat. Bei Männer wiederum eine leitenden Funktion im Beruf (odds 
ratio: $1,210^{* *}$ versus 1,053$)$ sowie die Vermögensausstattung an Wohneigentum (odds ratio: $1,429^{* * *}$ versus 1,394 ${ }^{* * *}$ ) von größerem Einfluss ist. Die Befunde von Marshall/Taniguchi (2012), dass sich bei Frauen eine Leitungsfunktion im Beruf positiv auf den Zugang zur Freiwilligenarbeit auswirkt, treffen somit in Österreich nicht zu.

Fügt man den Modellen noch Variablen zur Haushaltsgröße und zur Anzahl und zum Alter der im Haushalt gemeinsam lebenden Kinder hinzu, werden erwartungsgemäß Unterschiede zwischen den Geschlechtern sichtbar.

Die Haushaltsgröße wurde anhand der Anzahl der Personen die im gemeinsamen Haushalt leben gemessen sowie weiter spezifiziert, ob darunter auch Kinder unter 3 Jahren oder aber im Alter zwischen 3 und 15 Jahren sind. Die ersten beiden Modelle (Model I und II) in Tabelle 16 zeigen, dass die Hinzunahme der Variablen zur Haushaltstruktur den Einfluss des Familienstands zwar reduziert (von $1,396^{* * *}$ auf $1,240^{* * *}$ ), jedoch nichts an dessen Signifikanz und Wirkrichtung verändert. Der Familienstand erhöht demnach unabhängig von der Haushaltsgröße die Wahrscheinlichkeit für Freiwilliges Engagement. Sofern Kinder unter drei Jahren im gemeinsamen Haushalt wohnen, reduziert dies im Allgemeinen signifikant den Zugang zur Freiwilligenarbeit, und zwar um 33,2\% (odds ratio:,668 $\left.{ }^{* * *}\right)$. Lebt man gemeinsam mit Kinder im Alter zwischen 3 und 15 Jahren, fördert dies die Wahrscheinlichkeit für Freiwilligenarbeit (odds ratio: 1,131*). Den bestehenden Studien zufolge beeinflussen Kinder abhängig von deren Alter vor allem das Freiwillige Engagement von Frauen negativ (vgl. Musick/Wilson 2008: 245). Wie die Modelle III-VI zeigen, beeinflusst die Haushaltsgröße sowohl das Engagement von Frauen als auch von Männern positiv. Der negative Einfluss von Kindern unter 3 Jahren sowie der positive Einfluss von Kindern im Alter zwischen 3 und 15 Jahren zeigen sich hingegen nur bei Frauen als signifikant. Damit bestätigen sich die Befunde von Musick/Wilson (2008) aus den USA auch in Österreich. Bei den Männern verliert durch die Aufnahme der erweiterten Haushaltsdaten sogar der positive Einfluss des Familienstands an Bedeutung und ist nicht mehr signifikant. Alleine zu leben ist somit für Männer eine zentrale Barriere für Freiwilliges Engagement.

Zusammengefasst fördert das Zusammenleben in größeren Haushalten die Freiwilligenarbeit. Die Erklärungsansätze dazu sind vielfältig und reichen von der Reduktion der relativen Belastung durch Haushaltsaufgaben, sei es dadurch, dass sie von einer Person alleine getragen werden oder aber tatsächlich aufgeteilt werden, bis hin einer höheren Ressourcenausstattung (Einkommen, Bildungsgrad, Sozialkontakten, Urbanisierungsgrad, etc.) von sogenannten DINK Paaren (Dual Income No Kids). 
Tabelle 16: Logistische Regression - Formelle Freiwilligenarbeit: Frauen und Männer (inkl. Haushaltsdaten $)^{71}$

\begin{tabular}{|c|c|c|c|c|c|c|}
\hline & \multicolumn{6}{|c|}{ Abhängige Variable: Formelle Freiwilligenarbeit } \\
\hline & \multicolumn{2}{|c|}{ Gesamt } & \multicolumn{2}{|c|}{ Frauen } & \multicolumn{2}{|c|}{ Männer } \\
\hline & Model I & Model II & $\begin{array}{l}\text { Model } \\
\text { III }\end{array}$ & $\begin{array}{l}\text { Model } \\
\text { IV }\end{array}$ & $\begin{array}{l}\text { Model } \\
\text { V }\end{array}$ & $\begin{array}{c}\text { Model } \\
\text { VI }\end{array}$ \\
\hline & $\operatorname{Exp}(B)$ & $\operatorname{Exp}(B)$ & $\operatorname{Exp}(B)$ & $\operatorname{Exp}(B)$ & $\operatorname{Exp}(B)$ & $\operatorname{Exp}(B)$ \\
\hline Frauen (Ref. Männer) & $0,559^{* * *}$ & $0,557^{* * *}$ & & & & \\
\hline Alter & $0,990^{* * *}$ & $0,995^{* *}$ & $0,991^{* * *}$ & 0,996 & $0,990^{* * *}$ & 0,996 \\
\hline Geburtsland (Ref. Österreich) & $0,576^{* * *}$ & $0,575^{* * *}$ & $0,627^{* * *}$ & $0,631^{* * *}$ & $0,515^{* * *}$ & $0,510^{* * *}$ \\
\hline Bildungsgrad (1 bis 6) & $1,222^{* * *}$ & $1,241^{* * *}$ & $1,264^{* * *}$ & $1,292^{* * *}$ & $1,187^{* * *}$ & $1,199^{* * *}$ \\
\hline $\begin{array}{l}\text { Erwerbstätig (Ref. Erwerbslos, } \\
\text { Arbeitslos, in Pension, } \\
\text { Präsenz-oder Zivildiener) }\end{array}$ & 1,050 & 1,038 & 0,994 & 0,954 & 1,114 & 1,125 \\
\hline $\begin{array}{l}\text { Wohnrechtsverhältnis } \\
\text { Eigentum (Ref. Miete, } \\
\text { Untermiete, sonstiges) }\end{array}$ & $1,422^{* * *}$ & $1,290^{* * *}$ & $1,393^{* * *}$ & $1,266^{* * *}$ & $1,437^{* * *}$ & $1,301^{* * *}$ \\
\hline $\begin{array}{l}\text { Verheiratet (Ref. Ledig, } \\
\text { Geschieden, Verwitwet) }\end{array}$ & $1,396^{* * *}$ & $1,240^{* * *}$ & $1,470^{* * *}$ & $1,328^{* * *}$ & $1,336^{* * *}$ & 1,157 \\
\hline $\begin{array}{l}\text { Haushaltsgröße ( } 1 \text { bis } 9 \\
\text { Personen) }\end{array}$ & & $1,135^{* * *}$ & & $1,117^{* * *}$ & & $1,161^{* * *}$ \\
\hline $\begin{array}{l}\text { Kinder unter } 3 \text { Jahre im } \\
\text { Haushalt lebend }\end{array}$ & & $0,668^{* * *}$ & & $0,530^{* * *}$ & & 0,832 \\
\hline $\begin{array}{l}\text { Kinder zwischen } 3 \text { und } 15 \\
\text { Jahren im Haushalt lebend }\end{array}$ & & $1,131^{*}$ & & $1,292^{* * *}$ & & 0,975 \\
\hline Urbanisierungsgrad & $0,740^{* * *}$ & $0,755^{* * *}$ & $0,807^{* * *}$ & $0,822^{* * *}$ & $0,677^{* * *}$ & $0,693^{* * *}$ \\
\hline Konstante & $2,947^{* * *}$ & $1,719^{* * *}$ & 0,645 & $0,386^{* * *}$ & $2,280^{* * *}$ & 1,229 \\
\hline $\mathrm{N}$ & 11.657 & 11.657 & 6155 & 6155 & 5502 & 5502 \\
\hline $\mathrm{Chi}^{2} / \mathrm{df}$ & $931 / 8$ & $1024 / 11$ & $344 / 7$ & $426 / 10$ & $360 / 7$ & $393 / 10$ \\
\hline -2 Log-Likelihood & $14.874,40$ & 14781,541 & 7579,38 & 7497,34 & 7265,37 & 7232,12 \\
\hline Pseudo R² (Nagelkerke’s) & 0,103 & 0,113 & 0,075 & 0,092 & 0,084 & 0,092 \\
\hline
\end{tabular}

Quelle: Mikrozensus-Zusatzerhebung 2006/Q4; eigene Berechnung; $\left(p^{*}<0.05 ; p^{* *}<0.01 ; p^{* * *}<0.001\right)$.

71 Zur übersichtlicheren Darstellung werden nur die Odds ratios (Exp(B)) angeführt, nicht jedoch die Beta-Koeffizienten sowie die Standardfehler. 


\section{(III) 4.2.4. Determinanten von Mehrfachengagement}

Im Zusammenhang mit der Frage nach der Akkumulation von symbolischem wie sozialem Kapital ist auch von Bedeutung, in wie vielen Organisationen man im Rahmen der Freiwilligenarbeit tätig ist.

Tabelle 17: Häufigkeiten von Mehrfachengagement - Anzahl der Organisationen

\begin{tabular}{|c|c|c|c|c|c|c|}
\hline \multirow{2}{*}{$\begin{array}{l}\text { Anzahl der } \\
\text { Organisationen }\end{array}$} & \multicolumn{2}{|c|}{ Gesamt } & \multicolumn{2}{|c|}{ Männer } & \multicolumn{2}{|c|}{ Frauen } \\
\hline & $\mathbf{N}$ & $\%$ & $\mathbf{N}$ & $\%$ & $\mathbf{N}$ & $\%$ \\
\hline 1 & 2816 & 58,5 & 1509 & 56,0 & 1307 & 61,7 \\
\hline 2 & 1184 & 24,6 & 707 & 26,2 & 477 & 22,5 \\
\hline 3 & 459 & 9,5 & 259 & 9,6 & 200 & 9,4 \\
\hline 4 & 185 & 3,8 & 112 & 4,2 & 73 & 3,4 \\
\hline 5 & 90 & 1,9 & 58 & 2,2 & 32 & 1,5 \\
\hline 6 und mehr & 80 & 1,7 & 52 & 1,9 & 28 & 1,3 \\
\hline Gesamt & 4814 & 100,0 & 2697 & 100,0 & 2117 & 100,0 \\
\hline Mittelwert & 1,71 & & 1,76 & & 1,64 & \\
\hline Standardabweichung & 1,09 & & 1,13 & & 1,04 & \\
\hline
\end{tabular}

Quelle: Mikrozensus-Zusatzerhebung 2006/Q4; eigene Berechnungen.

Wie aus Tabelle 17 ersichtlich ist, üben mit 58,5\% etwas mehr als die Hälfte der Freiwilligen ihr Engagement in nur einer Organisation aus. Rund ein Viertel $(24,6 \%)$ ist in zwei und rund $17 \%$ in drei oder mehr Organisationen tätig. Vergleicht man die Verteilung betreffend der Organisationszugehörigkeit zwischen den Frauen und Männer zeigt sich, dass der Mittelwert bei den Männern mit 1,76 signifikant über jenem der Frauen mit 1,64 liegt ${ }^{72}$.

Für das nachfolgende Modell zur Analyse der Determinanten von Mehrfachengagement (Tabelle 18) wurde (vgl. Mood 2009) auf das Schätzverfahren der linearen Regression zurückgegriffen. Das Geschlecht und das Geburtsland zeigen dabei den gleichen Einfluss wie auch schon beim Zugang zur Freiwilligenarbeit. Eine Frau zu sein $\left(-0,093^{* *}\right)$ sowie nicht in Österreich geboren $\mathrm{zu}$ sein $\left(-0,180^{* *}\right)$ wirkt signifikant negativ auf Mehrfachengagement. Der Bildungsgrad $\left(0,080^{* * *}\right)$ hingegen steht in einem positiven Zusammenhang mit der Anzahl an Organisationen, ebenso wie der Familienstand verheiratet sein $\left(0,132^{* * \star}\right)$.

$72 \mathrm{t}(4693,550)=3,742, \mathrm{p}<, 001$ 
Die Erwerbstätigkeit im Allgemeinen hat keinen signifikanten Einfluss auf das Mehrfachengagement, eine leitenden Funktion im Beruf inne zu haben wirkt hingegen förderlich $\left(0,089^{*}\right)$.

Tabelle 18: Lineare Regression - Mehrfachengagement in der Freiwilligenarbeit

\begin{tabular}{|c|c|c|c|c|c|c|}
\hline & \multicolumn{6}{|c|}{$\begin{array}{c}\text { Abhängige Variable: Mehrfachengagement (1 bis 6+ } \\
\text { Organisationen) }\end{array}$} \\
\hline & \multicolumn{2}{|c|}{ Gesamt } & \multicolumn{2}{|c|}{ Männer } & \multicolumn{2}{|c|}{ Frauen } \\
\hline & B & SE & B & SE & B & SE \\
\hline Konstante & $1,723^{* * *}$ & 0,116 & $1,589^{* * *}$ & 0,155 & $1,564^{* * *}$ & 0,146 \\
\hline Frauen (Ref. Männer) & $-0,093^{* *}$ & 0,032 & & & & \\
\hline Alter & $0,003^{*}$ & 0,001 & 0,002 & 0,002 & $0,004^{*}$ & 0,002 \\
\hline Geburtsland (Ref. Österreich) & $-0,180^{* *}$ & 0,070 & $-0,087$ & 0,105 & $-0,268^{* *}$ & 0,091 \\
\hline Bildungsgrad & $0,080^{* * *}$ & 0,011 & $0,070^{* * *}$ & 0,015 & $0,096^{* * *}$ & 0,016 \\
\hline $\begin{array}{l}\text { Erwerbstätig (Ref. Erwerbslos, } \\
\text { Arbeitslos, in Pension, } \\
\text { Präsenz- oder Zivildiener) }\end{array}$ & 0,015 & 0,039 & 0,014 & 0,058 & 0,006 & 0,052 \\
\hline Leitenden Funktion im Beruf & $0,089^{*}$ & 0,041 & 0,090 & 0,052 & 0,079 & 0,071 \\
\hline $\begin{array}{l}\text { Wohnrechtsverhältnis } \\
\text { Eigentum (Ref. Miete, } \\
\text { Untermiete, sonstiges) }\end{array}$ & 0,034 & 0,039 & 0,049 & 0,054 & 0,017 & 0,055 \\
\hline $\begin{array}{l}\text { Verheiratet (Ref. Ledig, } \\
\text { Geschieden, Verwitwet) }\end{array}$ & $0,132^{* * *}$ & 0,037 & $0,178^{* * *}$ & 0,055 & $0,098^{*}$ & 0,050 \\
\hline Bevölkerungsdichte & $-0,114^{* * *}$ & 0,020 & $-0,117^{* * *}$ & 0,029 & $-0,112^{* * *}$ & 0,029 \\
\hline $\mathrm{R}^{2}$ & 0,031 & 1,072 & 0,026 & 1,113 & 0,034 & 1,019 \\
\hline $\mathrm{N}$ & 4.814 & & 2.697 & & 2.117 & \\
\hline
\end{tabular}

Quelle: Mikrozensus-Zusatzerhebung 2006/Q4; eigene Berechnung; $\left(p^{*}<0.05 ; p^{* *}<0.01 ; p^{* * *}<0.001\right)$.

Auf das Mehrfachengagement von Männern wirkt primär der Bildungsgrad und der Familienstand verheiratet förderlich. Bei den Frauen ist der positive Einfluss des Bildungsgrades mit $0,096^{* * *}$ stärker ausgeprägt als bei den Männer mit $0,070^{* * *}$. Nicht in Österreich geboren zu sein $\left(0,268^{* *}\right)$ ist nur für Frauen ein signifikant hinderlicher Faktor für Mehrfachengagement. Die Ergebnisse zeigen damit, dass kulturelles Kapital nicht nur für den Zugang zur Freiwilligenarbeit, sondern auch für Mehrfachengagement ein zentraler Einflussfaktor 
ist. Ökonomisches Kapitel hingegen ist primär für den generellen Zugang zur Freiwilligenarbeit mitentscheidend. Auf die Intensität der Freiwilligenarbeit in Form von Mehrfachengagement hat das Wohnrechtsverhältnis hingegen keine fördernde Wirkung und kann damit, wie schon angedeutet, auch als „Hygienefaktor" (vgl. Herzberg 1987) der Freiwilligenarbeit bezeichnet werden. Der Familienstand ist neben dem Bildungsgrad jener Einflussfaktor, dessen Einfluss sowohl auf den Zugang als auch auf Mehrfachengagement durchgehend signifikant ist. Dabei ist verheiratet zu sein förderlicher für das Engagement der Männer als das der Frauen.

Vor dem Hintergrund der Forschungsfrage und konkret vor der These einer sozialen Schließung in der Freiwilligenarbeit lassen sich die Ergebnisse folgendermaßen interpretieren. Einen erschwerten Zugang zur Freiwilligenarbeit haben insbesondere Personen die über ein geringes Ausmaß an kulturellem Kapital, d.h. über einen niedrigen Bildungsabschluss verfügen, die über eine vergleichsweise geringe Ausstattung an ökonomischem Kapital (in Form von Wohneigentum) verfügen und alleine leben. Da davon auszugehen ist, dass mit der Anzahl der Organisationen, in denen man freiwillig engagiert ist, auch die Größe des sozialen Netzwerkes, vor allem der weak ties (Granovetter 1973), positiv zusammenhängt, dürfte es durch die ungleiche Verteilung von Mehrfachengagement auch zu einer ungleichen Akkumulation von sozialem und symbolischem Kapital in der Freiwilligenarbeit kommen. Es ist davon auszugehen, dass Reputationsgewinnen durch Freiwilliges Engagement zu weiterem Engagement führen (Matthäus-Effekt), wie dies z.B. bei ehrenamtlichen Vorstandpositionen oftmals zu beobachten ist.

\section{(III) 4.2.5. Determinanten des Zugangs der bildungsfernsten Schicht}

Vor dem Hintergrund der Bestrebungen der gesellschaftlichen Integration von sozial benachteiligten Bevölkerungsgruppen durch Freiwilliges Engagement sind jene Faktoren von Interesse die konkret das Freiwillige Engagement der bildungsfernsten Gruppe, die ja mitunter die niedrigste Beteiligungsquote aufweist, beeinflussen. Da besonders bei dieser Gruppe große Unterschiede zwischen den Geschlechtern zu erwarten sind, erfolgt die Analyse getrennt nach Männer und Frauen. 
Tabelle 19: Logistische Regression - Formelle Freiwilligenarbeit von Personen mit höchstens Pflichtschulabschluss getrennt nach Frauen und Männer

\begin{tabular}{|c|c|c|c|c|c|c|}
\hline & \multicolumn{6}{|c|}{ Abhängige Variable: Formelle Freiwilligenarbeit } \\
\hline & \multicolumn{3}{|c|}{$\begin{array}{c}\text { Frauen (Bildungsgrad } \\
=<\text { Pflichtschule) }\end{array}$} & \multicolumn{3}{|c|}{$\begin{array}{l}\text { Männer (Bildungsgrad } \\
=<\text { Pflichtschule) }\end{array}$} \\
\hline & Beta & SE & $\operatorname{Exp}(B)$ & Beta & SE & $\operatorname{Exp}(B)$ \\
\hline Alter & $-0,019$ & 0,003 & $0,981^{* * *}$ & $-0,014$ & 0,005 & $0,986^{* *}$ \\
\hline Geburtsland (Ref. Österreich) & $-0,423$ & 0,266 & 0,655 & $-0,682$ & 0,319 & $0,505^{*}$ \\
\hline $\begin{array}{l}\text { Erwerbstätig (Ref. Erwerbslos, } \\
\text { Arbeitslos, in Pension, Präsenz- oder } \\
\text { Zivildiener) }\end{array}$ & $-0,284$ & 0,131 & $0,753^{* *}$ & $-0,020$ & 0,157 & 0,980 \\
\hline $\begin{array}{l}\text { Wohnrechtsverhältnis Eigentum (Ref. } \\
\text { Miete, Untermiete, sonstiges) }\end{array}$ & 0,063 & 0,139 & 1,065 & 0,626 & 0,180 & $1,869^{* * *}$ \\
\hline $\begin{array}{l}\text { Verheiratet (Ref. Ledig, Geschieden, } \\
\text { Verwitwet) }\end{array}$ & 0,379 & 0,132 & $1,461^{* *}$ & 0,118 & 0,215 & 1,125 \\
\hline Bevölkerungsdichte & $-0,208$ & 0,080 & $0,812^{*}$ & $-0,263$ & 0,099 & $0,769^{* *}$ \\
\hline Konstante & 0,489 & 0,381 & 1,630 & 0,917 & 0,471 & 2,502 \\
\hline $\mathrm{N}$ & & 1544 & & & 782 & \\
\hline $\mathrm{Chi}^{2} / \mathrm{df}$ & & $51 / 6$ & & & $51 / 6$ & \\
\hline -2 Log-Likelihood & & 1694,4 & & & 1014,7 & \\
\hline Pseudo R² (Nagelkerke’s) & & 0,048 & & & 0,085 & \\
\hline
\end{tabular}

Quelle: Mikrozensus-Zusatzerhebung 2006/Q4; eigene Berechnung; $\left(p^{*}<0.05 ; p^{* *}<0.01 ; p^{* *}<0.001\right)$.

Die Beteiligungsquote von Frauen der untersten Bildungsschicht liegt mit 25,0\% deutlich unter jener der Männer mit 42,3\%. Damit scheint primär der erschwerte Zugang von Frauen dieser Bildungsschicht für die geringe Beteiligungsquote dieser Gruppe verantwortlich zu sein. Aus umgekehrter Perspektive stellt sich die Frage, über welche Kapitalien jene Personen verfügen, die trotz einer vergleichsweise geringen Ausstattung an kulturellem Kapital Zugang zur formellen Freiwilligenarbeit finden.

Anhand des Modells in Tabelle 19 wird sichtbar, dass für Frauen der bildungsfernsten Schicht eine Erwerbstätigkeit (odds ratio: $0,753^{* *}$ ) hinderlich, verheiratet zu sein (odds ratio: $1,461^{* *}$ ) jedoch in hohem Maße förderlich auf freiwilliges Engagement wirkt. Die ethnische Herkunft (odds ratio: 0,655) und das ökonomische Kapital (in Form von Wohneigentum; odds ratio: 1,065) haben keinen signifikanten Einfluss auf den Zugang zur formellen Freiwilligenarbeit von Frauen. Ob vor dem Hintergrund klassischer Geschlechterrollenstereotypen verheiratet sein bei Frauen weiterhin als ökonomische Absicherung zu 
interpretieren ist, kann nicht beantwortet werden. Bei den Männern hat die Tatsache, nicht in Österreich geboren zu sein, mit einer odds ratio von, $505^{*}$ einen signifikant negativen Einfluss auf die Freiwilligenarbeit und reduziert die Chance um fast 50\%. Die Erwerbstätigkeit hat weder einen signifikant negativen noch eine positiven Einfluss auf das Freiwillige Engagement von Männern der bildungsfernsten Schicht. Das ökonomische Kapital (Wohneigentum) hingegen erhöht signifikant die Wahrscheinlichkeit für Freiwilligenarbeit bei den Männern und zwar um $86,9 \%$ (odds ratio: $1,869^{* * *}$ ). Verheiratet zu sein wiederum hat keinen signifikanten Einfluss. Zusammengefasst, dürfte es damit vor allem für unverheiratete, erwerbstätige Frauen der untersten Bildungsschicht besonders schwierig sein, Zugang zu formeller Freiwilligenarbeit zu finden. Ihre Beteiligungsquote ist mit 23,3\% etwa nur halb so hoch wie jener der unverheirateten, erwerbstätigen Männer mit 44,7\%. Demgegenüber dürfte es vorrangig für Männer der bildungsfernsten Schicht, die nicht über Wohneigentum und damit einer basalen ökonomischen Absicherung verfügen und/oder nicht in Österreich geboren sind, im besonderen Maße schwierig sein, sich in der Freiwilligenarbeit zu engagieren. Für Frauen der untersten Bildungsschicht scheint es nur in den wenigsten Fällen möglich zu sein Erwerbsarbeit und Freiwilligenarbeit zu verbinden, was darauf hindeutet, dass es sich bei dieser Gruppe bei der Erwerbsarbeit und der Freiwilligenarbeit um konfliktäre Kontextebenen handelt (vgl. Diewald/Faist 2011: 106).

\section{(III) 4.2.6. Subjektive Einschätzung der Barrieren der Freiwilligenarbeit}

Im Rahmen der Mikrozensus-Zusatzerhebung (siehe Fragebogen im Anhang) zur Freiwilligenarbeit wurden die Personen, die angegeben haben, weder formelle noch informelle Freiwilligenarbeit zu leisten, nach den Gründen gefragt. Die Respondenten mussten bei zehn vorgegeben Aussagen beurteilen, ob diese jeweils auf sie zutreffen oder nicht (vgl. BMASK 2009: 224).

Die größte Zustimmung bei klassisch externalen Gründen haben familiäre Aufgaben (72,1\%), nicht gefragt worden sein $(60,1 \%)$ und zeitliche Unvereinbarkeit mit dem Beruf (45,5\%). Eine geringe Zustimmung haben internale Gründe wie nicht darüber nachgedacht zu haben $(39,6 \%)$ oder das Gefühl, keinen nützlichen Beitrag leisten zu können (9,3\%), gefunden. Gesundheitliche (18,6\%) und ökonomische $(15,1 \%)$ Gründe werden ebenfalls nur von einem kleinen Teil der Befragten als Hindernisse angesehen. 
Tabelle 20: Gründe sich nicht ehrenamtlich bzw. freiwillig zu engagieren

\begin{tabular}{|c|c|c|c|c|}
\hline Vorgegebene Aussagen & $\begin{array}{l}\text { trifft } \mathrm{zu} \\
\quad \%\end{array}$ & $\begin{array}{c}\text { Gültig } \\
\mathrm{n}\end{array}$ & $\begin{array}{c}\text { keine } \\
\text { Antwort } \\
\mathrm{n}\end{array}$ & $\begin{array}{c}\text { weiß } \\
\text { nicht } \\
\mathrm{n}\end{array}$ \\
\hline „Ich bin durch familiäre Aufgaben ausgefüllt" & 72,1 & 4562 & 19 & 11 \\
\hline "Ich bin niemals gefragt und gebeten worden" & 60,1 & 4566 & 17 & 9 \\
\hline $\begin{array}{l}\text { „Es lässt sich mit meinem Beruf zeitlich nicht } \\
\text { vereinbaren“" }\end{array}$ & 45,5 & 4564 & 21 & 6 \\
\hline „Ich habe nie darüber nachgedacht" & 39,6 & 4563 & 18 & 11 \\
\hline $\begin{array}{l}\text { „Es gibt in meiner Nähe keine für mich attrakti- } \\
\text { ven Möglichkeiten, ehrenamtlich zu arbeiten" }\end{array}$ & 24,6 & 4475 & 22 & 94 \\
\hline $\begin{array}{l}\text { "Ich fühle mich durch Krankheit oder } \\
\text { Behinderung nicht in der Lage“" }\end{array}$ & 18,6 & 4567 & 17 & 7 \\
\hline „Das ist nichts für meine Altersgruppe“ & 16,7 & 4544 & 23 & 24 \\
\hline $\begin{array}{l}\text { „Ich kann mir das nicht leisten, da es für mich } \\
\text { mit zu hohen Kosten verbunden ist“ }\end{array}$ & 15,1 & 4549 & 21 & 21 \\
\hline $\begin{array}{l}\text { "Ich habe das Gefühl, dass ich keinen nützlichen } \\
\text { Beitrag leisten kann“" }\end{array}$ & 9,3 & 4548 & 22 & 21 \\
\hline „Ich habe schlechte Erfahrungen gemacht“ & 5,7 & 4564 & 22 & 5 \\
\hline
\end{tabular}

Quelle: Mikrozensus-Zusatzerhebung 2006/Q4; eigene Berechnung.

Für die weitere multivariate Analyse wurden folgenden vier Antworten ausgewählt. (I) Die Aussage „Ich kann mir das nicht leisten, da es für mich mit zu hohen Kosten verbunden ist" kann dabei der ökonomischen Sphäre zugeordnet werden. (II) „Ich habe nie darüber nachgedacht" kann vor dem Hintergrund der Forschung zum Zusammenhang von Bildungsgrad und Freiwilligenarbeit der kulturellen Sphäre zugeordnet werden. (III) Die Aussage „Ich bin niemals gefragt und gebeten worden" entspringt der Sphäre der sozialen Netzwerke. (IV) „Ich habe das Gefühl, dass ich keinen nützlichen Beitrag leisten kann"kann einerseits der Sphäre des Selbstwerts und der Selbstwirksamkeit (subjektivistisch) zugeordnet werden und begründet sich andererseits auch aus dem zugeschriebenen sozialen Status (objektivistisch).

Analysiert man die Zustimmungsquoten zu den vier ausgewählten Antworten nach den zentralen sozialen Merkmalsgruppen zeigt sich, dass hier teils beträchtliche Unterschiede bestehen. 
Tabelle 21: Logistische Regression - Gründe für kein freiwilliges Engagement

\begin{tabular}{|c|c|c|c|c|}
\hline & \multicolumn{4}{|c|}{ Abhängige Variablen: } \\
\hline & $\begin{array}{l}\text { Modell I } \\
\text { „Ich bin } \\
\text { niemals } \\
\text { gefragt und } \\
\text { gebeten } \\
\text { worden" } \\
(60,1 \%)\end{array}$ & $\begin{array}{l}\text { Modell II } \\
\text { „Ich habe } \\
\text { nie darüber } \\
\text { nachgedacht“ } \\
(39,6 \%)\end{array}$ & $\begin{array}{l}\text { Modell III } \\
\text { „Ich kann } \\
\text { mir das } \\
\text { nicht leisten, } \\
\text { da es für } \\
\text { mich mit zu } \\
\text { hohen Kosten } \\
\text { verbunden } \\
\text { ist“ }(15,1 \%)\end{array}$ & $\begin{array}{l}\text { Modell IV } \\
\text { „Ich habe das } \\
\text { Gefühl, dass } \\
\text { ich keinen } \\
\text { nützlichen } \\
\text { Beitrag } \\
\text { leisten kann" } \\
(9,3 \%)\end{array}$ \\
\hline Frauen (Ref. Männer) & $1,350^{* * *}$ & 0,896 & $1,250^{*}$ & 0,905 \\
\hline Alter & $0,984^{* * *}$ & $0,989^{* * *}$ & $1,007^{*}$ & $1,029^{* * *}$ \\
\hline Geburtsland (Ref. Österreich) & 1,052 & $1,280^{*}$ & $1,919^{* * *}$ & $1,572^{* *}$ \\
\hline Bildungsgrad ( 1 bis 6 ) & $0,946^{*}$ & $0,821^{* * *}$ & $0,769^{* * *}$ & $0,713^{* * *}$ \\
\hline $\begin{array}{l}\text { Erwerbstätig (Ref. } \\
\text { Erwerbslos, Arbeitslos, in } \\
\text { Pension, Präsenz-oder } \\
\text { Zivildiener) }\end{array}$ & $0,808^{* *}$ & 0,868 & $0,455^{* * *}$ & $0,493^{* * *}$ \\
\hline $\begin{array}{l}\text { Wohnrechtsverhältnis } \\
\text { Eigentum (Ref. Miete, } \\
\text { Untermiete, sonstiges) }\end{array}$ & 0,883 & $0,821^{* *}$ & $0,619^{* * *}$ & $0,679^{* * *}$ \\
\hline $\begin{array}{l}\text { Verheiratet (Ref. Ledig, } \\
\text { Geschieden, Verwitwet) }\end{array}$ & 1,132 & 1,027 & 0,855 & 0,968 \\
\hline $\begin{array}{l}\text { Haushaltsgröße ( } 1 \text { bis } 9 \\
\text { Personen) }\end{array}$ & $0,886^{* * *}$ & 0,984 & 1,013 & 0,901 \\
\hline Urbanisierungsgrad & $1,166^{* * *}$ & 0,992 & 0,998 & 0,984 \\
\hline Konstante & $2,602^{* * *}$ & $2,049^{* *}$ & $0,167^{* *}$ & $0,084^{* * *}$ \\
\hline $\mathrm{N}$ & 4566 & 4563 & 4549 & 4548 \\
\hline $\mathrm{Chi}^{2} / \mathrm{df}$ & $116 / 9$ & $118 / 9$ & $291 / 9$ & $366 / 9$ \\
\hline -2 Log-Likelihood & 6026,599 & 6009,631 & 3564,149 & 2457,588 \\
\hline Pseudo $\mathrm{R}^{2}$ (Nagelkerke's) & 0,034 & 0,035 & 0,108 & 0,167 \\
\hline
\end{tabular}

Quelle: Mikrozensus-Zusatzerhebung 2006/Q4; eigene Berechnung; $\left(p^{*}<0.05 ; p^{* *}<0.01 ; p^{* * *}\right.$ $<0.001)$.

Bivariat (siehe Tabelle 44 im Anhang) zeigt sich, dass „Niemals gefragt oder gebeten worden sein" für mehr als die Hälfte der Befragten zutrifft. Überdurchschnittlich trifft dies wiederum auf die Gruppe der 15- bis 29-Jährigen zu (65,5\%), Personen die nicht in Österreich geboren sind (62,8\%), jene die mit Kindern unter 3 Jahren zusammenwohnen $(64,2 \%)$ sowie in Miete $(64,8 \%)$ leben. Eine hohe 
Bevölkerungsdichte erhöht ebenfalls die Zustimmung (65,0\%). Die bivariaten Zusammenhänge bestätigen sich auch im multivariaten Modell I (siehe Tabelle 21). Die Chance, dass Frauen als Grund angeben, „Niemals gefragt oder gebeten worden sein“, ist um 35\% (odds ratio: 1,350**) höher als bei Männern. „Niemals gefragt oder gebeten worden sein" trifft darüber hinaus eher auf jüngere Personen mit niedrigerem Bildungsgrad zu, die nicht erwerbstätig sind, eher alleine und in der Stadt leben. Die Chance nicht gefragt zu werden, reduziert sich mit jeder Person mehr im Haushalt um 11,4\% (odds ratio: 0,886 $6^{* * *}$ ). Die Ergebnisse bestätigen somit die These, dass die subjektive Barriere „Niemals gefragt oder gebeten worden sein" mit der Höhe an sozialem Kapital (Haushaltsgröße und/oder Beruf) und kulturellem Kapital (Bildungsgrad) zusammenhängt (vgl. Musick/ Wilson 2008: 120, 126).

„Nie darüber nachgedacht zu haben" findet überdurchschnittlich hohe $\mathrm{Zu}$ stimmung bei Personen zwischen 15 und 29 Jahren (48,0\%), bei Personen nicht österreichischer Herkunft (44,6\%), Personen mit höchstens Pflichtschulabschluss (44,6\%) und Lehrabschluss (43,3\%), bei Personen die in Miete leben (43,3\%) (siehe Tabelle $44 \mathrm{im}$ Anhang). Dies bestätigt sich auch im multivariaten Modell (II) (siehe Tabelle 21). "Nie darüber nachgedacht zu haben" trifft eher auf jüngere Menschen zu, die nicht in Österreich geboren sind, über ein geringeres Bildungsniveau verfügen und eher in Miete als in Eigentum leben. Mit diesem Ergebnis finden gleich mehrere Thesen ihre Bestätigung. Erstens, dass für Personen, die nicht in Österreich geboren und vielfach von einem anderen Kulturkreis geprägt sind, die tradierten Formen des formellen Engagements wie sie in Österreich üblich sind seltener optionale Handlungsfelder darstellen. Bei dieser Personengruppe sind vielfach informelle Formen der Freiwilligenarbeit verstärkt anzutreffen (vgl. Reinprecht 2009). Zweitens bestätigen sich die Befunde, dass mit einer geringeren Ausstattung an kulturellem Kapital vielfach ein geringeres Problembewusstsein für zivilgesellschaftliche Anliegen einhergeht (vgl. Campbell 2009; Gesthuizen/Scheepers 2012).

"Ich kann mir das nicht leisten, da es für mich mit zu hohen Kosten verbunden ist", d.h. ein Mangel an ökonomischem Kapital ist für Frauen (16,9\%) bedeutsamer als für Männer (12,5\%) ebenso wie für Personen zwischen 50 und 64 Jahren $(17,7 \%)$ und der Gruppe der über 65-Jährigen (23,7\%) (siehe Tabelle 44 im Anhang). Rund ein Viertel der Personen, die nicht in Österreich geboren sind, geben an, dass Freiwilliges Engagement für sie mit zu hohen Kosten verbunden ist. Ähnliche Ergebnisse finden sich bei der bildungsfernsten Schicht, d.h. jene mit höchstens Pflichtschulabschluss (24,8\%), der Gruppe der Nicht-Erwerbstätigen $(23,3 \%)$ und jenen, die in Miete $(19,5 \%)$ leben. Auch bei den ökonomischen Barrieren bestätigen sich die Einflussfaktoren im multivariaten Modell (III) (siehe 
Tabelle 21). Die Chance, dass Erwerbstätige dieser Aussage zustimmen, ist um rund 55\% geringer als bei Erwerbslosen, die Chance von in Eigentum Lebenden ist um 38\% geringer als bei jenen, die in Miete leben. Damit findet sich auch in diesem Zusammenhang die These bestätigt, dass man sich formelle Freiwilligenarbeit tatsächlich leisten können muss. Personen mit einer vergleichsweise geringen Ausstattung an ökonomischem Kapital (MigrantInnen, bildungsferne Schichten, Erwerbslose und in Miete lebende Personen) nehmen diese Barriere auch selbst als solche wahr.

"Keinen nützlichen Beitrag leisten zu können" findet vor allem bei Personen über 65 Jahren überdurchschnittlich hohe Zustimmung (24,7\%). Ebenso bei Personen, die nicht in Österreich geboren sind $(13,4 \%)$, die höchstens über einen Pflichtschulabschluss verfügen $(16,6 \%)$ sowie nicht erwerbstätige Personen (16,7\%) (siehe Tabelle 44 im Anhang). In Miete zu leben erhöht ebenfalls die Chance für das Gefühl keinen Beitrag leisten zu können. Dieses Bild wird auch im multivariaten Modell IV bestätigt. Das Gefühl keinen Beitrag leisten zu können, wird signifikant durch das Alter, das Geburtsland, den Bildungsgrad, die Erwerbslosigkeit und das Wohnrechtsverhältnis beeinflusst. Bourdieus These zur "Grausamkeit" der Verteilung von gesellschaftlicher Anerkennung (vgl. Bourdieu 2001b: 309f) findet in diesen Ergebnissen ihren Widerhall. Jene Personen, denen vergleichsweise wenig soziale Anerkennung zu Teil wird, wie Migrantinnen und Migranten, Erwerbs- und Arbeitslosen und Angehörigen bildungsferner Schichten, erleben sich selbst vergleichsweise häufiger als nutzlos.

\section{(III) 4.2.7. Zusammenfassung der Ergebnisse zur sozialen Schließung}

Die multivariate Analyse hat den bivariat beobachtbaren Einfluss der askriptiven Merkmale (Geschlecht, Alter, ethnische Herkunft) auf den Zugang zur Freiwilligenarbeit in Österreich bestätigt. Frauen, ältere Personen sowie Migrantinnen und Migranten sind seltener freiwillig engagiert und sind somit sozialen Schließungstendenzen in der Freiwilligenarbeit ausgesetzt. Hinsichtlich der erworbenen Merkmale (Bildungsgrad, beruflichen Stellung und Vermögen) zeigt sich ein etwas differenziertes Bild: Ein höherer Bildungsgrad wirkt uneingeschränkt förderlich auf den Zugang. Demgegenüber steht eine Erwerbstätigkeit nur dann mit einer freiwilligen Tätigkeit in positivem Zusammenhang, wenn damit auch eine Leitungsfunktion verbunden ist. Die Vermögensausstattung in Form von Wohneigentum hat sich als zentraler Einflussfaktor für den Zugang zur Freiwilligenarbeit in Österreich herausgestellt. Verheiratet zu sein bildet vielfach ebenso die soziale und finanzielle Basis für Freiwilliges Engagement. Darüber hinaus ist Freiwilliges Engagement in Regionen mit einem geringeren Urbanisierungsgrad 
häufiger anzutreffen als in großen Städten. Ergänzend hat der Vergleich der Determinanten des Zugangs zur Freiwilligenarbeit mit jenen des Zugangs zur Erwerbsarbeit gezeigt, dass sich abgesehen von der Vermögensausstattung an Wohneigentum, die Zugangskriterien zu den beiden Tätigkeitsfeldern zwar hinsichtlich ihrer Einflussstärke, nicht jedoch in ihrer grundlegenden Wirkrichtung und Bedeutung unterscheiden. Erwerbsarbeit und Freiwilligenarbeit stellen damit, zumindest betreffend ihre Zugangsregeln vorwiegend kongruente und nicht komplementäre bzw. kompensatorische Tätigkeitsfelder dar.

Die Gegenüberstellung der Determinanten des Engagements von Männern und Frauen hat deutlich gemacht, dass betreffend der weiteren askriptiven Merkmale (Alter und ethnische Herkunft) keine bemerkenswerten Unterschiede bestehen. Bei den erworbenen Merkmalen hat sich gezeigt, dass für Frauen der Bildungsgrad und der Familienstand und bei Männer eine leitenden Funktion im Beruf und die Vermögensausstattung an Wohneigentum mehr Bedeutung für den Zugang zur Freiwilligenarbeit haben. Die Anzahl der Personen, die im gemeinsamen Haushalt leben, hat bei Männern und Frauen einen positiven Einfluss auf das Engagement. Handelt es sich dabei um Kinder unter 3 Jahren, wirkt sich das primär negativ auf das Freiwillige Engagement von Frauen aus. Sind die Kinder zwischen 3 und 15 Jahren alt, fördert dies wiederum vorwiegend das Engagement von Frauen.

Mehr als 40\% der Freiwilligen engagieren sich in mehr als einer Organisation ehrenamtlich. Die dafür förderlichen Ressourcen bzw. Kapitalien umfassen einen höheren Bildungsgrad, eine leitende Funktion im Beruf und einen Ehepartner. Frauen sowie Migrantinnen und Migranten sind seltener in mehreren Organisationen freiwillig tätig als Männer sowie in Österreich geborene Personen. Die Vermögensausstattung in Form von Wohneigentum spielt für Mehrfachengagement keine bedeutsame Rolle.

Ein Blick auf die Determinanten des Engagements der untersten Bildungsschicht hat gezeigt, dass bei Frauen und Männern unterschiedliche Ressourcen einen Einfluss haben. Für Frauen dieser Gruppe ist neben dem Alter vor allem eine Erwerbstätigkeit hinderlich, wohingegen das Leben in Ehe förderlich auf das Freiwillige Engagement wirkt. Bei Männern hingegen ist neben dem Alter primär nicht in Österreich geboren zu sein ein hinderlicher Faktor. Verfügen Männer der untersten Bildungsschicht über Wohneigentum fördert das in hohem Ausmaß deren Freiwilliges Engagement.

Die Analyse der subjektiven Barrieren der Freiwilligenarbeit und deren Determinanten hat offen gelegt, dass sich die "subjektiv“ wahrgenommenen Barrieren vielfach mit der "objektiv“ nötigen Ressourcenausstattung bzw. den Kapitalien für den Zugang zur Freiwilligenarbeit decken. Die in den sozialen, 
ökonomischen, kulturellen und symbolischen Dimensionen benachteiligten Bevölkerungsgruppen nehmen ihre diesbezügliche Schlechterstellung auch subjektiv als Barrieren beim Zugang zur Freiwilligenarbeit wahr. Sofern als gesellschaftspolitisches Ziel definiert, gilt es für die vermehrte gesellschaftliche Integration dieser Bevölkerungsgruppen, den Zugang zu besagten Kapitalien zu fördern und die Integration in die Freiwilligenarbeit als Indikator für das Gelingen zu interpretieren. Gelungene Sozialpolitik sollte sich somit, ceteris paribus, positiv auf den Abbau von Barrieren und auf die Beteiligung aller Bevölkerungsschichten an der Freiwilligenarbeit auswirken.

Gesamt betrachtet ist damit zu erwarten, dass die soziale Schließung in der Freiwilligenarbeit zu einer Verfestigung und teilweisen Verstärkung der sozialen Ungleichheit beiträgt und nicht, wie vielfach angenommen, Freiwilliges Engagement ein kompensatorisches Handlungsfeld darstellt.

\section{(III) 4.3. Multivariate Analysen zur internen Hierarchisierung in der Freiwilligenarbeit}

In diesem Kapitel steht die Analyse des Zugangs zu leitenden Positionen in der Freiwilligenarbeit im Zentrum: Welchen Einfluss haben askriptive wie erworbene soziale Merkmale (Geschlecht, Alter, ethnische Herkunft, Bildungsgrad, Vermögen, berufliche Stellung und Prestige) auf den Zugang zu Positionen mit Leitungsfunktion in der Freiwilligenarbeit in Österreich? Diese Frage wird ergänzt durch die Perspektiven auf die Statusreproduktion in der Freiwilligenarbeit: In welcher Form kommt es in der Freiwilligenarbeit zu einer Reproduktion von Prestige und Status der Erwerbsarbeit? Dazu folgen im Anschluss Analysen zum Vergleich der Hierarchisierung in der Freiwilligenarbeit und der Erwerbsarbeit; zum Einfluss von Mehrfachengagement auf die Position innerhalb der Freiwilligenarbeit; zu den Unterschieden zwischen Männern und Frauen; sowie zum Einfluss des Berufsprestiges. Abschließend wird vor dem Hintergrund der Statusreproduktion der bivariate Zusammenhang von (Erwerbs-)Arbeitszeit und Freiwilligenarbeit analysiert.

\section{(III) 4.3.1. Determinanten des Zugangs zu Positionen mit} Leitungsfunktion

Die Scherengrafiken, wie sie im einleitenden Kapitel zu den bivariaten Ergebnissen dargestellt wurden, zeigen die Bedeutung des Geschlechts, des Bildungsgrades, der Erwerbstätigkeit und des Familienstandes für den Zugang zu Positionen mit Leitungsfunktion in der Freiwilligenarbeit. Die ethnische Herkunft und die Vermögensausstattung (Wohnrechtsverhältnis) scheinen aufgrund der schon 
beim Zugang zur Freiwilligenarbeit erfolgten Selektion und sozialen Schließung für die interne hierarchische Strukturierung und Positionsbesetzung keinen Einfluss mehr zu haben.

Tabelle 22: Logistische Regression - Leitende Funktion in der Freiwilligenarbeit

\begin{tabular}{|c|c|c|c|}
\hline \multirow[t]{2}{*}{ Modell I } & \multicolumn{3}{|c|}{$\begin{array}{l}\text { Abhängige Variable: Leitende Funktion in der } \\
\text { Freiwilligenarbeit }\end{array}$} \\
\hline & Beta & SE & $\operatorname{Exp}(B)$ \\
\hline Frauen (Ref. Männer) & $-0,670$ & 0,073 & $0,512^{* * *}$ \\
\hline Alter & 0,011 & 0,003 & $1,011^{* * *}$ \\
\hline Geburtsland (Ref. Österreich) & 0,200 & 0,146 & 1,221 \\
\hline Bildungsgrad & 0,192 & 0,023 & $1,212^{* * *}$ \\
\hline $\begin{array}{l}\text { Erwerbstätig (Ref. Erwerbslos, } \\
\text { Arbeitslos, in Pension, Präsenz- oder } \\
\text { Zivildiener) }\end{array}$ & 0,213 & 0,092 & $1,238^{*}$ \\
\hline Leitenden Funktion im Beruf & 0,348 & 0,083 & $1,416^{* * *}$ \\
\hline $\begin{array}{l}\text { Wohnrechtsverhältnis Eigentum } \\
\text { (Ref. Miete, Untermiete, sonstiges) }\end{array}$ & 0,014 & 0,085 & 1,014 \\
\hline $\begin{array}{l}\text { Verheiratet (Ref. Ledig, Geschieden, } \\
\text { Verwitwet) }\end{array}$ & 0,423 & 0,083 & $1,527^{*+*}$ \\
\hline Bevölkerungsdichte & $-0,072$ & 0,045 & 0,931 \\
\hline Konstante & $-1,779$ & 0,259 & 0,169 \\
\hline $\mathrm{N}$ & & 4.812 & \\
\hline $\mathrm{Chi}^{2} / \mathrm{df}$ & & $333 / 9$ & \\
\hline -2 Log-Likelihood & & 5258,339 & \\
\hline Pseudo R² (Nagelkerke’s) & & 0,097 & \\
\hline
\end{tabular}

Quelle: Mikrozensus-Zusatzerhebung 2006/Q4; eigene Berechnung; $\left(p^{*}<0.05 ; p^{* *}<0.01 ; p^{* * *}<0.001\right)$.

Das Regressionsmodell in Tabelle 22 bestätigt diesbezüglich die bivariaten Zusammenhänge. Frauen haben im Vergleich zu Männern eine um 48,8\% (odds ratio: $\left.0,512^{* * *}\right)$ geringere Chance, in eine Positionen mit Leitungsfunktion in der Freiwilligenarbeit zu kommen. Das Alter hingegen hat einen positiven Einfluss (odds ratio: $1,011^{* * *}$ ) und deutet darauf hin, dass Seniorität auch in der Freiwilligenarbeit Leitungsfunktionen kennzeichnet. Kulturelles Kapital in Form des Bildungsgrads (odds ratio: $1,212^{* * *}$ ) erhöht ebenfalls, und dabei unabhängig von der beruflichen Position, die Wahrscheinlichkeit, Leitungsaufgaben in der Freiwilligenarbeit zu 
übernehmen bzw. übertragen zu bekommen. Im Unterschied zum Zugang zur Freiwilligenarbeit, wo die Erwerbstätigkeit im multivariaten Modell keinen signifikanten Einfluss hat, wirkt sich eine berufliche Tätig signifikant positiv auf den Zugang zu Leitungsfunktionen in der Freiwilligenarbeit aus (odds ratio: 1,239*). Damit bestätigen die Ergebnisse die These von Ehrhardt (2011), nach der „Menschen mit einem inferioren Status (z.B. Arbeitslose) kein >mittleres oder gehobenes Engagement $<$ beginnen können, da sie nicht die Zugangsbedingungen dafür erfüllen" (Ehrhardt 2011: 64). Ob Personen, wie Ehrhardt weiter vermutet, auch aus einem solchen Ehrenamt ausscheiden, wenn sie arbeitslos werden, kann anhand der vorliegenden Daten nicht beantwortet werden. Der berufliche Status hat ebenfalls einen zentralen Einfluss auf die hierarchische Position in der Freiwilligenarbeit: Eine leitende Funktion im Beruf inne zu haben, erhöht die Wahrscheinlichkeit, in der Freiwilligenarbeit ebenfalls leitend tätig zu sein, um 41,6\% (odds ratio: $1,416^{* * *}$ ). Das Ergebnis zum Wohneigentum (odds ratio: 1,014) bestätigt die These: Vermögen ist für den Zugang zentral, jedoch in weiterer Folge nicht für die interne hierarchische Strukturierung. Hier ist vor allem die kulturelle, soziale und symbolische Kapitalausstattung von Bedeutung. Darüber hinaus hat der Familienstand verheiratet einen signifikant positiven Einfluss auf die Wahrscheinlichkeit eine leitenden Position inne zu haben (odds ratio: 1,527 $7^{* * *}$ ). Die Regeln in den Felder, in denen die Freiwilligenarbeit geleistet wird, scheinen jedenfalls dafür zu sorgen, dass sich der soziale wie berufliche Status mit den Positionen, die die Personen bei ihrer freiwilligen Tätigkeit im jeweiligen institutionellen Gefüge inne haben, tendenziell in Deckung bringen (vgl. Ehrhardt 2011: 62f).

\section{(III) 4.3.2. Determinanten des Zugangs zu Positionen mit Leitungsfunktion in der Freiwilligenarbeit und Erwerbsarbeit im Vergleich}

Beim Vergleich des Zugangs zu Leitungsfunktionen zeigt sich eine ähnliche Tendenz wie zuvor beim Vergleich des allgemeinen Zugangs zur Erwerbsarbeit und zur Freiwilligenarbeit (siehe Tabelle 23). Bis auf die ethnische Herkunft decken sich auch hier die Wirkrichtungen und Signifikanzen der Determinanten. Der Zugang zu Positionen mit Leitungsfunktion ist sowohl in der Erwerbsarbeit als auch in der Freiwilligenarbeit für Frauen, Personen mit niedrigerem Bildungsgrad und jene, die nicht verheiratet sind, unwahrscheinlicher. In der Erwerbarbeit haben zusätzlich noch Personen, die nicht in Österreich geboren sind, eine geringere Wahrscheinlichkeit (58,5\%) mit Leitungsaufgaben betraut zu werden als in Österreich geborene Personen. Die Regeln der internen Hierarchisierung nach Geschlecht, Alter und Bildungsgrad (kulturellem Kapital), wie sie in der 
Erwerbsarbeit wirksam sind, gelten auch für die interne vertikale Strukturierung der Freiwilligenarbeit. Ein gravierender Unterschied besteht jedoch bislang zwischen diesen beiden „Arbeitsfeldern“. In der Erwerbsarbeit wird zumindest versucht, in Teilbereichen durch Gleichstellungsagenden und Quotenregelung zur positiven Diskriminierung, bestimmten ungleichheitsgenerierenden Mechanismen entgegenzuwirken. In der Freiwilligenarbeit finden derartige Maßnahmen bislang nur als konjunktivierte Willenserklärung in politischen Dokumenten zum Jahr der Freiwilligenarbeit ${ }^{73}$ ihren Ausdruck bzw. werden vereinzelt von Organisationen, die in diesem Bereich eine besondere Schieflage aufweisen oder aber die Integration explizit zum Ziel haben, thematisiert.

Tabelle 23: Logistische Regression - Determinanten des Zugangs zu Positionen mit Leitungsfunktion in der Erwerbsarbeit und der Freiwilligenarbeit

\begin{tabular}{|c|c|c|c|c|c|c|}
\hline & \multicolumn{3}{|c|}{$\begin{array}{l}\text { Abhängige Variable: } \\
\text { Erwerbsarbeit } \\
\text { - Leitende Funktion }\end{array}$} & \multicolumn{3}{|c|}{$\begin{array}{l}\text { Abhängige Variable: } \\
\text { Formelle } \\
\text { Freiwilligenarbeit } \\
\text { - Leitende Funktion }\end{array}$} \\
\hline & Beta & SE & $\operatorname{Exp}(B)$ & Beta & SE & $\operatorname{Exp}(B)$ \\
\hline Frauen (Ref. Männer) & $-1,003$ & 0,036 & $0,367^{* * *}$ & $-0,770$ & 0,070 & $0,463^{*+*+}$ \\
\hline Alter & 0,030 & 0,002 & $1,031^{* * *}$ & 0,007 & 0,003 & $1,007^{* *}$ \\
\hline Geburtsland (Ref. Österreich) & $-0,904$ & 0,064 & $0,405^{*+*}$ & 0,147 & 0,146 & 1,158 \\
\hline Bildungsgrad & 0,310 & 0,012 & $1,364^{* * *}$ & 0,221 & 0,023 & $1,247^{*+*+}$ \\
\hline $\begin{array}{l}\text { Wohnrechtsverhältnis Eigentum } \\
\text { (Ref. Miete, Untermiete, sonstiges) }\end{array}$ & 0,061 & 0,041 & 1,062 & 0,018 & 0,085 & 1,018 \\
\hline $\begin{array}{l}\text { Verheiratet (Ref. Ledig, } \\
\text { Geschieden, Verwitwet) }\end{array}$ & 0,188 & 0,040 & $1,207^{* * *}$ & 0,513 & 0,081 & $1,671^{* *+x}$ \\
\hline Bevölkerungsdichte & $-0,039$ & 0,022 & 0,962 & $-0,072$ & 0,045 & 0,931 \\
\hline Konstante & $-0,816$ & 0,118 & $0,442^{*+*}$ & $-1,304$ & 0,230 & $0,271^{* * *}$ \\
\hline $\mathrm{N}$ & & 20.104 & & & 4.812 & \\
\hline $\mathrm{Chi}^{2} / \mathrm{df}$ & & $2247 / 7$ & & & $299 / 7$ & \\
\hline -2 Log-Likelihood & & 20506,853 & & & 5291,681 & \\
\hline Pseudo R² (Nagelkerkes) & & 0,156 & & & 0,088 & \\
\hline
\end{tabular}

Quelle: Mikrozensus-Zusatzerhebung 2006/Q4; eigene Berechnung; $\left(p^{*}<0.05 ; p^{* *}<0.01 ; p^{* * *}<0.001\right)$.

73 „Das, Europäische Jahr der Freiwilligentätigkeit ' zur Förderung der aktiven Bürgerschaft könnte [Hervorh. PR] auch dazu beitragen, geschlechterbezogene Ungleichheiten im Freiwilligensektor anzugehen, beispielsweise [...] in Bezug auf Repräsentation in ehrenamtlichen Führungspositionen“" (Der Rat der Europäischen Union 2010: L17/44). 
Wie bereits im Kapitel zum allgemeinen Zugang (sozialer Schließung) zur Freiwilligenarbeit wird auch an dieser Stelle die „Gegenprobe“ zum Einfluss der Freiwilligenarbeit auf den Zugang zu leitenden Positionen in der Erwerbsarbeit gemacht. Dabei zeigt sich, dass die Freiwilligenarbeit im Allgemeinen keinen signifikanten Einfluss auf den Zugang zu leitenden Funktionen in der Erwerbsarbeit hat (siehe Tabelle 48 im Anhang). Eine leitende Funktion in der Freiwilligenarbeit steht jedoch in einem signifikant positiven Zusammenhang (odds ratio: $\left.1,461^{* * *}\right)$ mit einer leitenden Funktion im Beruf. Dieses Ergebnis kann zum Teil dadurch erklärt werden, dass sich durch die Hinzuahme der Variablen zur Freiwilligenarbeit die Samplegröße auf jene Befragten reduziert hat, die an der Erhebung zur Freiwilligenarbeit teilgenommen haben. Durch die bereits beschriebenen Selektionseffekte ist anzunehmen, dass Personen mit Leitungsfunktionen im Beruf und einer Affinität zu Freiwilligenarbeit überrepräsentiert sind. Ergänzt man das vorangegangene Modell zur Erklärung des Zugangs zu leitenden Positionen in der Erwerbsarbeit um die Frage nach dem Einfluss der einzelnen Bereiche der Freiwilligenarbeit (siehe Tabelle 47 im Anhang) zeigt sich, dass primär das Freiwillige Engagement im Feld des Sports $\left(1,221^{*}\right)$ förderlich wirkt und ein Engagement im Feld der Kirche und Religion $\left(0,742^{* *}\right)$ einen negativen Zusammenhang mit einer leitenden Funktion im Beruf aufweist. Die Frage nach der tatsächlichen Kausalität kann jedoch mangels Längsschnittdaten nicht beantwortet werden.

\section{(III) 4.3.3. Mehrfachengagement und der Zugang zu Positionen mit Leitungsfunktion}

Die Einflussfaktoren auf Mehrfachengagement wurden bereits in Kapitel (III) 4.2.4. untersucht. Als signifikante Determinanten haben sich das Geschlecht, das Alter, das Geburtsland, der Bildungsgrad, eine leitende Funktion im Beruf und verheiratet zu sein herausgestellt. Fügt man die Variable Mehrfachengagement dem Regressionsmodell zur Erklärung des Zugangs zu leitenden Funktion in der Freiwilligenarbeit hinzu (Tabelle 24), zeigt sich, dass dieses signifikant die Wahrscheinlichkeit (odds ratio: 1,661 $1^{* * *}$ ), leitend tätig zu sein, erhöht. Das Pseudo $\mathrm{R}^{2}$ (Nagelkerkes) erhöht sich von 0,097 im Ausgangsmodell (Tabelle 22) auf 0,176 im erweiterten Modell (Tabelle 24). 
Tabelle 24: Logistische Regression - Leitenden Funktion in der Freiwilligenarbeit (inkl. Mehrfachengagement)

\begin{tabular}{|c|c|c|c|}
\hline & \multicolumn{3}{|c|}{$\begin{array}{l}\text { Abhängige Variable: Leitende Funktion } \\
\text { in der Freiwilligenarbeit }\end{array}$} \\
\hline & Beta & SE & $\operatorname{Exp}(B)$ \\
\hline Frauen (Ref. Männer) & $-0,671$ & 0,075 & $0,511^{* * *}$ \\
\hline Alter & 0,010 & 0,003 & $1,010^{* * *}$ \\
\hline Geburtsland (Ref. Österreich) & 0,316 & 0,150 & $1,372^{*}$ \\
\hline Bildungsgrad & 0,159 & 0,024 & $1,172^{* * *}$ \\
\hline $\begin{array}{l}\text { Erwerbstätig (Ref. Erwerbslos, } \\
\text { Arbeitslos, in Pension, Präsenz-oder } \\
\text { Zivildiener) }\end{array}$ & 0,210 & 0,094 & $1,234^{*}$ \\
\hline Leitenden Funktion im Beruf & 0,328 & 0,087 & $1,388^{* * *}$ \\
\hline $\begin{array}{l}\text { Wohnrechtsverhältnis Eigentum } \\
\text { (Ref. Miete, Untermiete, sonstiges) }\end{array}$ & 0,001 & 0,088 & 1,001 \\
\hline $\begin{array}{l}\text { Verheiratet (Ref. Ledig, Geschieden, } \\
\text { Verwitwet) }\end{array}$ & 0,366 & 0,085 & $1,442^{* * *}$ \\
\hline Bevölkerungsdichte & $-0,004$ & 0,047 & 0,996 \\
\hline Mehrfachengagement ( 1 bis $6+$ ) & 0,507 & 0,031 & $1,661^{* * *}$ \\
\hline Konstante & $-2,733$ & 0,274 & $0,065^{* * *}$ \\
\hline $\mathrm{N}$ & & 4812 & \\
\hline $\mathrm{Chi}^{2} / \mathrm{df}$ & & $418 / 10$ & \\
\hline -2 Log-Likelihood & & 4972,375 & \\
\hline Pseudo R² (Nagelkerke’s) & & 0,176 & \\
\hline
\end{tabular}

Quelle: Mikrozensus-Zusatzerhebung 2006/Q4; eigene Berechnung; $\left(p^{*}<0.05 ; p^{* *}<0.01 ; p^{* * *}<0.001\right)$.

Zum Beispiel wird der Einfluss der ethnischen Herkunft in dem Maße signifikant, dass Nicht-Österreicher nun eine 37,2\% erhöhte Wahrscheinlichkeit beim Zugang zu leitenden Funktionen haben als in Österreich geborene Personen. Dieser Effekt entsteht dadurch, dass der erschwerte Zugang von nicht in Österreich geborenen Personen bereits im Mehrfachengagement latent abgebildet ist.

\section{(III) 4.3.4. Determinanten des Zugangs von Frauen und Männer zu Positionen mit Leitungsfunktion im Vergleich}

Der Zugang von Frauen zu Positionen mit Leitungsfunktion in der Freiwilligenarbeit ist gegenüber Männern analog zur Erwerbsarbeit 
deutlich eingeschränkt. $68,6 \%$ der Leitungspositionen sind in der Freiwilligenarbeit von Männern besetzt, nur 31,4\% von Frauen und das, obwohl $44 \%$ der Freiwilligen Frauen sind. Die vertikale bzw. hierarchische Geschlechtersegregation setzt sich damit in der Freiwilligenarbeit fort.

Tabelle 25: Logistische Regression - Leitende Funktion in der Freiwilligenarbeit getrennt nach Frauen und Männer

\begin{tabular}{|c|c|c|c|c|c|c|}
\hline & \multicolumn{6}{|c|}{$\begin{array}{l}\text { Abhängige Variable: Leitende Funktion } \\
\text { in der Freiwilligenarbeit }\end{array}$} \\
\hline & \multicolumn{3}{|c|}{ Frauen } & \multicolumn{3}{|c|}{ Männer } \\
\hline & Beta & SE & $\operatorname{Exp}(B)$ & Beta & SE & $\operatorname{Exp}(B)$ \\
\hline Alter & 0,009 & 0,005 & 1,009 & 0,012 & 0,004 & $1,012^{* * *}$ \\
\hline Geburtsland (Ref. Österreich) & 0,216 & 0,213 & 1,241 & 0,186 & 0,200 & 1,204 \\
\hline Bildungsgrad & 0,226 & 0,039 & $1,254^{* * *}$ & 0,173 & 0,030 & $1,189^{* * *}$ \\
\hline $\begin{array}{l}\text { Erwerbstätig (Ref. Erwerbslos, } \\
\text { Arbeitslos, in Pension, } \\
\text { Präsenz-oder Zivildiener) }\end{array}$ & 0,198 & 0,138 & 1,219 & 0,207 & 0,123 & 1,230 \\
\hline Leitenden Funktion im Beruf & 0,188 & 0,166 & 1,207 & 0,400 & 0,099 & $1,492^{* * *}$ \\
\hline $\begin{array}{l}\text { Wohnrechtsverhältnis } \\
\text { Eigentum (Ref. Miete, } \\
\text { Untermiete, sonstiges) }\end{array}$ & 0,001 & 0,142 & 1,001 & 0,025 & 0,107 & 1,026 \\
\hline $\begin{array}{l}\text { Verheiratet (Ref. Ledig, } \\
\text { Geschieden, Verwitwet) }\end{array}$ & 0,312 & 0,129 & $1,367^{*}$ & 0,481 & 0,109 & $1,617^{* * *}$ \\
\hline Bevölkerungsdichte & $-0,003$ & 0,073 & 0,997 & $-0,119$ & 0,058 & $0,888^{*}$ \\
\hline Konstante & $-3,137$ & 0,380 & $0,043^{* * *}$ & $-2,403$ & 0,317 & $0,090^{* * *}$ \\
\hline $\mathrm{N}$ & \multicolumn{3}{|c|}{2117} & \multicolumn{3}{|c|}{2696} \\
\hline $\mathrm{Chi}^{2} / \mathrm{df}$ & \multicolumn{3}{|c|}{$63 / 8$} & \multicolumn{3}{|c|}{$160 / 8$} \\
\hline -2 Log-Likelihood & \multicolumn{3}{|c|}{2000,63} & \multicolumn{3}{|c|}{3250,28} \\
\hline Pseudo R² (Nagelkerke’s) & \multicolumn{3}{|c|}{0,047} & \multicolumn{3}{|c|}{0,080} \\
\hline
\end{tabular}

Quelle: Mikrozensus-Zusatzerhebung 2006/Q4; eigene Berechnung; $\left(p^{*}<0.05 ; p^{* *}<0.01\right.$; $\left.p^{* * *}<0.001\right)$.

Die Regressionsmodelle in Tabelle 25 zeigen, dass die erklärenden Variablen den Zugang von Frauen zu Positionen mit Leitungsfunktion mit einem Pseudo $R^{2}$ von 0,047 weniger gut erklären, als den Zugang von Männern mit einem Pseudo $\mathrm{R}^{2}$ von 0,080 . Für den Zugang zu leitenden Funktionen ist bei Frauen der Bildungsgrad (odds ratio 1,254 ${ }^{* * *}$ ) und verheiratet $\mathrm{zu}$ sein (odds ratio: 
$1,367^{*}$ ) entscheidend. Bei den Männern wirkt das Alter (odds ratio: 1,012 ${ }^{* * *}$ ), der Bildungsgrad (odds ratio: $1,189^{* * *}$ ), eine leitenden Funktion im Beruf (odds ratio: $1,492^{* * *}$ ) und verheiratet zu sein (odds ratio: $1,617^{* * *}$ ), signifikant auf die hierarchische Strukturierung in der Freiwilligenarbeit. Somit ist kulturelles Kapital in Form von Bildungstiteln sowie der Familienstand sowohl für Männer als auch für Frauen ein zentraler Faktor der vertikalen Strukturierung in der Freiwilligenarbeit. Eine leitende Funktion im Beruf als Indikator für symbolisches Kapital des Feldes der Erwerbsarbeit ist hingegen primär für Männer ein zentraler Faktor.

In einem letzten Schritt wird noch der Einfluss erwerbs- bzw. berufsspezifischer Faktoren wie der berufliche Status (ISEI) sowie von Teilzeit- und Vollzeitanstellungen auf die hierarchische Position in der Freiwilligenarbeit analysiert.

\section{(III) 4.3.5. Beruflicher Status (ISEI) als Determinante bei Männern und Frauen}

Für die nachfolgende Analyse wurde das Sample auf die zum Zeitpunkt der Erhebung berufstätigen Freiwilligen reduziert (3.334 von 4.812 Respondenten) und die Regressionsmodelle um die Variable zum Zeitausmaß der Anstellung (Vollzeit/Teilzeit) sowie um den Berufsstatus (ISEI) erweitert. Aufgrund der Freiwilligkeit bei der Teilnahme an der Zusatzerhebung zur Freiwilligenarbeit beinhaltet der Datensatz nicht die vollständigen Angaben aller Haushaltsmitglieder. Im vorliegenden Fall kann nicht-erwerbstätigen Personen, nicht wie sonst üblich der Berufsstatus des erwerbstätigen Partners zugeordnet werden, sondern lediglich jenen Personen ein ISEI (sozio-ökonomischer Status) zugeordnet werden, die zum Zeitpunkt der Erhebung Angaben zu ihrer Erwerbstätigkeit gemacht haben. Die Größe des in die Regressionsanalyse einbezogenen Samples reduziert sich daher nachfolgend bei den Frauen auf N=1.345 $(63,5 \%)$ und bei Männer auf N=1.989 (73,8\%).

Für erwerbstätige und freiwillig tätige Frauen ist der Bildungsgrad (odds ratio: $1,179^{* * *}$ ) entscheidend, ob sie Zugang zu einer Position mit Leitungsfunktion finden oder nicht. Eine Vollzeitanstellung und/oder eine leitenden Funktion im Beruf sind dafür nicht ausschlaggebend. Bei den erwerbstätigen männlichen Freiwilligen hingegen erhöht das Alter (odds ratio: 1,013*), der Bildungsgrad (odds ratio: $1,117^{* *}$ ), die Höhe des Berufsstatus (ISEI) (odds ratio: 1,009*), eine leitende Funktion im Beruf (odds ratio: 1,396 $6^{* * *}$ und ein Ehepartner $\left(1,819^{* * *}\right.$ ) die Wahrscheinlichkeit eine Position mit Leitungsfunktion in der Freiwilligenarbeit inne zu haben. 
Tabelle 26: Logistische Regression - Leitende Funktion in der Freiwilligenarbeit der Erwerbstätigen getrennt nach Frauen und Männer

\begin{tabular}{|c|c|c|c|c|c|c|}
\hline & \multicolumn{6}{|c|}{$\begin{array}{l}\text { Abhängige Variable: Leitende Funktion in der } \\
\text { Freiwilligenarbeit }\end{array}$} \\
\hline & \multicolumn{3}{|c|}{ Frauen } & \multicolumn{3}{|c|}{ Männer } \\
\hline & Beta & SE & $\operatorname{Exp}(B)$ & Beta & SE & $\operatorname{Exp}(B)$ \\
\hline Alter & 0,013 & 0,007 & 1,013 & 0,013 & 0,005 & $1,013^{* *}$ \\
\hline Geburtsland (Ref. Österreich) & 0,288 & 0,284 & 1,333 & 0,183 & 0,246 & 1,201 \\
\hline Bildungsgrad & 0,165 & 0,058 & $1,179^{+*+*}$ & 0,110 & 0,042 & $1,117^{* *}$ \\
\hline Teilzeit (Ref. Vollzeit>35h) & $-0,039$ & 0,143 & 0,962 & $-0,171$ & 0,186 & 0,843 \\
\hline Berufsstatus (ISEI) & 0,008 & 0,005 & 1,008 & 0,009 & 0,004 & $1,009^{*}$ \\
\hline Leitenden Funktion im Beruf & 0,150 & 0,169 & 1,162 & 0,333 & 0,103 & $1,396^{* * *}$ \\
\hline $\begin{array}{l}\text { Wohnrechtsverhältnis Eigentum } \\
\text { (Ref. Miete, Untermiete, sonstiges) }\end{array}$ & $-0,105$ & 0,179 & 0,900 & $-0,020$ & 0,126 & 0,980 \\
\hline $\begin{array}{l}\text { Verheiratet (Ref. Ledig, Geschieden, } \\
\text { Verwitwet) }\end{array}$ & 0,214 & 0,166 & 1,239 & 0,598 & 0,124 & $1,819^{* * *+}$ \\
\hline Bevölkerungsdichte & $-0,106$ & 0,090 & 0,899 & $-0,185$ & 0,068 & $0,831^{* *}$ \\
\hline Konstante & $-3,003$ & 0,484 & $0,050^{* * *}$ & $-2,347$ & 359 & $0,096^{*+*}$ \\
\hline $\mathrm{N}$ & & 1345 & & & 1989 & \\
\hline $\mathrm{Chi}^{2} / \mathrm{df}$ & & $38 / 9$ & & & $133 / 9$ & \\
\hline -2 Log-Likelihood & & 1341,225 & & & 2433,948 & \\
\hline Pseudo R² (Nagelkerke’s) & & 0,043 & & & 0,089 & \\
\hline
\end{tabular}

Quelle: Mikrozensus-Zusatzerhebung 2006/Q4; eigene Berechnung; $\left(p^{*}<0.05 ; p^{* *}<0.01 ; p^{* * *}<0.001\right)$.

Diese Ergebnisse lassen sich dahingehend interpretieren, dass sich in der Freiwilligenarbeit primär die männerdominierten Regeln der Erwerbsarbeit reproduzieren und es dadurch implizit wie explizit zu einer Reproduktion gesamtgesellschaftlicher Ungleichheit, z.B. betreffend der Kopplung von Leitungsfunktionen an Bildungstitel kommt. Darüber hinaus, kommt es in der Freiwilligenarbeit durch die Kopplung von Leitungsfunktionen an Leitungsfunktionen in der Erwerbsarbeit und an den beruflichen Status zu einer weiteren Akkumulation von symbolischem Kapital, d.h. konkret von Anerkennung, Prestige und Macht sowie von sozialem Kapital, d.h. von bedeutsamen Sozialkontakten, insbesondere von weak ties (vgl. Granovetter 1973). Im Sinne Bourdieus Analysen zur Arbeitsteilung in der katholischen Kirche (vgl. Bourdieu 1998), ist auch in der Freiwilligenarbeit davon auszugehen, dass zumindest in Teilbereichen verschleierte, d.h. in die Doxa des jeweiligen Feldes eingeschriebene und 
zur Natürlichkeit gewordene, Ausbeutungsverhältnissen existieren. Freiwillige aus benachteiligten sozialen Gruppen müssen so meist scheinbar ihren (vergleichsweise geringen) Fähigkeiten und Kompetenzen entsprechend, mit einfachen Tätigkeiten und Funktionen vorlieb nehmen. Der politisch verkündete Kompetenzerwerb durch Freiwilliges Engagement beschränkt sich damit vorwiegend auf bereits "gut ausgestatte" Bevölkerungsgruppen und folgt damit dem Prinzip des Matthäus-Effekts (DiPrete/Eirich 2006; Merton 2010).

Zum Abschluss wird noch ein Blick auf den vielfach kontraintuitiven $\mathrm{Zu}$ sammenhang der Arbeitszeit in der Erwerbsarbeit und der Freiwilligenarbeit geworfen.

\section{(III) 4.3.6. „Paradox der Gestreßtheit“: Zum Zusammenhang von Arbeitszeit und Freiwilligenarbeit}

Dieses Kapitel geht der Frage nach, in welcher Form und in welchem Ausmaß sich das Bourdieu'sche „Paradox der Gestreßtheit" und Überbeanspruchung der Privilegierten auch in der Freiwilligenarbeit wieder findet. Vergleicht man in einem ersten Schritt (Tabelle 27) die Beteiligungsquote an der Freiwilligenarbeit von Teilzeitbeschäftigten $(43,5 \%)$ mit jenen von Vollzeitbeschäftigen (44,9\%), zeigt sich ein nur geringfügiger Unterschied von 1,4 Prozentpunkten. Personen die keiner erwerbstätigen Beschäftigung nachgehen, weisen hingegen eine deutlich niedrigere Beteiligungsquote von 35,5\% auf. Betrachtet man die Leitungsquote, d.h. z.B. den Anteil an Freiwilligen mit Teilzeitbeschäftigung, die in der Freiwilligenarbeit eine Position mit Leitungsfunktion bekleiden, zeigt sich, dass $22 \%$ der teilzeitbeschäftigten und 31,6\% der vollzeitbeschäftigten Freiwilligen eine leitende Funktion innehaben. Der Unterschied beträgt in diesem Fall zugunsten der Vollzeiterwerbstätigen fast 10 Prozentpunkte.

Tabelle 27: Beteiligungsquoten - Formelle Freiwilligenarbeit und Leitungsfunktion in der Freiwilligenarbeit nach Teilzeit- oder Vollzeitbeschäftigung

\begin{tabular}{lcccccc}
\hline & \multicolumn{2}{c}{$\begin{array}{c}\text { Beteiligungsquote } \\
\text { formelle Freiwilligenarbeit }\end{array}$} & & \multicolumn{2}{c}{$\begin{array}{c}\text { Leitungsquote in der } \\
\text { Freiwilligenarbeit }\end{array}$} \\
\cline { 2 - 3 } \cline { 5 - 6 } & $\mathbf{N}$ & \% & & $\mathbf{N}$ & \% \\
\hline Teilzeit bis 35 h/Wo & 1.984 & 43,5 & & 864 & 22,0 \\
Vollzeit ab 36 h/Wo & 5.457 & 44,9 & & 2.449 & 31,6 \\
stark schwankend & 47 & 44,7 & & 21 & 33,3 \\
\hline Gesamt & 7.488 & 44,5 & & 3.334 & 29,1 \\
\hline
\end{tabular}

Quelle: Mikrozensus-Zusatzerhebung 2006/Q4; eigene Berechnung. 
Wie sich jedoch bereits im Kapitel zur sozialen Schließung gezeigt hat, ist das Beschäftigungsausmaß im multivariaten Modell nicht signifikant. Das Regressionsmodell in Tabelle 28 bestätigt die These, dass hinter der geringen Leitungsquote von Teilzeitbeschäftigten (Tabelle 27) die geringe Chance von Frauen (odds ratio:, $531^{* * *}$ ) steht, Zugang zu Positionen mit Leitungsfunktion in der Freiwilligenarbeit zu finden.

Tabelle 28: Logistische Regression - Einfluss von Beschäftigungsausmaß und Geschlecht beim Zugang zu leitenden Positionen in der Freiwilligenarbeit

\begin{tabular}{|c|c|c|c|c|c|c|}
\hline & \multicolumn{6}{|c|}{$\begin{array}{l}\text { Abhängige Variable: Leitende Funktion } \\
\text { in der Freiwilligenarbeit }\end{array}$} \\
\hline & \multicolumn{3}{|c|}{ Modell I } & \multicolumn{3}{|c|}{ Modell II } \\
\hline & Beta & SE & $\operatorname{Exp}(B)$ & Beta & SE & $\operatorname{Exp}(B)$ \\
\hline Teilzeit (Ref. Vollzeit>35h) & $-0,494$ & 0,092 & $0,610^{* * *}$ & $-0,145$ & 0,106 & 0,865 \\
\hline Frauen (Ref. Männer) & & & & $-0,633$ & 0,094 & $0,531^{* * *}$ \\
\hline Konstante & $-0,771$ & 0,043 & $0,463^{* * *}$ & 0,010 & 0,122 & 1,010 \\
\hline $\mathrm{N}$ & & 3334 & & & 3334 & \\
\hline $\mathrm{Chi}^{2} / \mathrm{df}$ & & $30 / 1$ & & & $77 / 2$ & \\
\hline -2 Log-Likelihood & & 3990,695 & & & 3943,402 & \\
\hline Pseudo R² (Nagelkerke’s) & & 0,013 & & & 0,033 & \\
\hline
\end{tabular}

Quelle: Mikrozensus-Zusatzerhebung 2006/Q4; eigene Berechnung; $\left(p^{*}<0.05 ; p^{* *}<0.01 ; p^{* * *}<0.001\right)$.

In einem nächsten Schritt (Tabelle 29) wird nun die durchschnittliche Wochenarbeitszeit von Freiwilligen mit ausführenden und administrativen Funktionen mit jener der Freiwilligen mit Leistungsfunktion verglichen. Die durchschnittliche normale berufliche Wochenarbeitszeit von erwerbstätigen Freiwilligen mit Leitungsfunktion ist mit 42,8 Std. deutlich höher als die von erwerbstätigen Freiwilligen ohne Leitungsfunktion mit 38,5 Std (Tabelle 29). Die mittlere Wochenarbeitszeit der Freiwilligen mit Leitungsfunkton in der Freiwilligenarbeit liegt damit mit 4,3 Stunden signifikant über jener der Freiwilligen ohne Leitungsfunktion. 
Tabelle 29: Normale Wochenarbeitsstunden in der Erwerbsarbeit von Freiwilligen mit und ohne Leitungsfunktion im Vergleich

\begin{tabular}{lcc}
\hline & $\begin{array}{c}\text { Freiwillige in } \\
\text { ausführender/ } \\
\text { administrativer/ } \\
\text { sonstiger Funktion }\end{array}$ & $\begin{array}{c}\text { Freiwillige in leitender } \\
\text { Funktion }\end{array}$ \\
\hline N Gültig & 2350 & 963 \\
Fehlend & 14 & 7 \\
Mittelwert & $38,541^{* *+}$ & $42,825^{* * *}$ \\
Median & 40,000 & 40,000 \\
Standardabweichung & 15,526 & 16,220 \\
Standardfehler des Mittelwertes & 0,320 & 0,523 \\
Minimum & 0,75 & 0,50 \\
Maximum & 120,00 & 126,00 \\
\hline
\end{tabular}

Grundgesamtheit: nur Erwerbstätige - N=3.335.

Quelle: Mikrozensus-Zusatzerhebung 2006/Q4; eigene Berechnung; Mittelwertwergleich (T-Test für unabhängige Stichproben; $p^{*}<0.05 ; p^{* *}<0.01 ; p^{* * *}<0.001$ ).

Fasst man die Wochenarbeitszeit zu Stundenkategorien zusammen und setzt diese in Beziehung zum Anteil an Freiwilligen mit Leitungsfunktion (Abbildung 18), zeigt sich, dass mit der Höhe der Wochenarbeitszeit auch der Anteil der Personen

Abbildung 18: Zusammenhang der Wochenarbeitszeit (Std.) in der Erwerbsarbeit (Referenzwoche) mit der Leitungsfunktion in der Freiwilligenarbeit

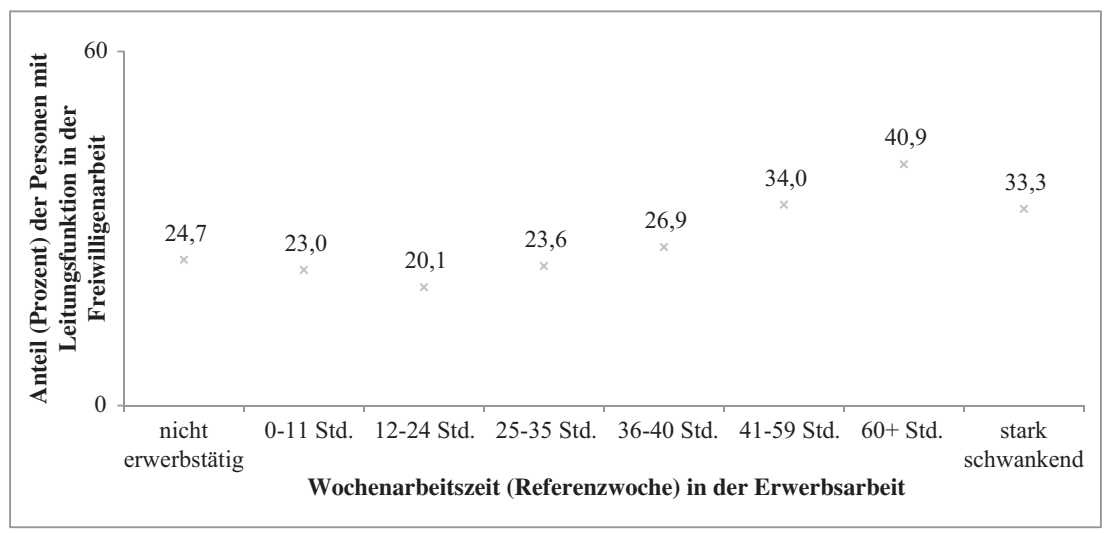

Quelle: Mikrozensus-Zusatzerhebung 2006/Q4; eigene Berechnung; N=4812. 
mit Leitungsfunktion steigt. Rund 23\% der Freiwilligen, die wöchentlich zwischen 0-11 Stunden erwerbstätig sind, haben eine ehrenamtliche Leitungsfunktion inne, wohingegen rund $41 \%$ der Freiwilligen die 60 und mehr Stunden pro Woche ihrer Erwerbstätigkeit nachgehen im Ehrenamt in Leitender Funktion sind.

Diese Ergebnisse deuten darauf hin, dass die These Bourdieus (2001b: 291) zur "Gestreßtheit der Privilegierten" auch auf das Engagement in der Freiwilligenarbeit zutrifft. Sie verweisen damit zum wiederholten Male auf die mit der Freiwilligenarbeit verbundenen symbolischen und sozialen Profite, ohne die sich ein Engagement der privilegierten Schichten nicht „lohnen“ würde.

\section{(III) 4.3.7. Zusammenfassung der Ergebnisse zur Hierarchisierung}

Die bivariaten Befunde zu den Determinanten des Zugangs zu leitenden Positionen der Freiwilligenarbeit haben sich auch in der multivariaten Analyse bestätigt. Die askriptiven Merkmale Geschlecht und Alter beeinflussen die interne hierarchische Strukturierung in den Freiwilligenorganisationen. Frauen haben eine um fast die Hälfte geringere Chance in ehrenamtliche Leitungspositionen zu kommen. Die ethnische Herkunft hat dabei keinen Einfluss mehr. Bei den erworbenen Merkmalen (Bildungsgrad, berufliche Stellung, berufliches Prestige, Vermögensausstattung und Familienstand) sind der Bildungsgrad, die Erwerbstätigkeit und dabei in besonderem Maße eine leitende Funktion sowie das Leben in einer ehelichen Partnerschaft von zentraler Bedeutung. Die Vermögensausstattung in Form von Wohneigentum verliert im Rahmen der internen Hierarchisierung ihre Bedeutung.

Der Vergleich der Determinanten der Hierarchisierung in der Freiwilligenarbeit mit jenen in der Erwerbsarbeit macht deutlich, dass die beiden Tätigkeitsfelder nicht nur über ähnliche Zugangsregeln, sondern ebenso über ähnliche Regeln der internen Strukturierung verfügen. Hinsichtlich des Geburtslandes ist die Erwerbsarbeit weniger selektiv beim allgemeinen Feldzugang, jedoch umso selektiver bei der Auswahl von Leitungspositionen. Umgekehrtes trifft auf die Freiwilligenarbeit zu. Hat man es als nicht in Österreich geborener in die Freiwilligenarbeit geschafft, bildet das Geburtsland alleine kein weiteres Selektionskriterium beim Zugang zu ehrenamtlichen Leitungsfunktionen. Hingegen stellt ein höherer Bildungsgrad in beiden Feldern, sowohl beim Zugang als auch bei der Positionsbesetzung einen zentralen Faktor dar. Der Familienstand „verheiratet" ist in beiden Feldern von Bedeutung, jedoch in der Freiwilligenarbeit ungleich förderlicher für leitende Positionen. Zusammengefasst sind Erwerbsarbeit und Freiwilligenarbeit auch betreffend der Regeln der internen Positionsbesetzung vergleichbare Kontextebenen.

Personen, die in mehreren Organisationen freiwillig tätig sind, sind auch häufiger in einer oder mehreren leitenden Funktionen zu finden. Die diesbezüglichen 
Kausalitäten sind ohne entsprechenden Daten (z.B. Längsschnitterhebungen) nicht zu entflechten. Sowohl Mehrfachengagement als auch leitende Positionen verlangen nach einer vergleichsweise hohen Ausstattung an kulturellem und symbolischem Kapital. Es ist daher anzunehmen, dass hier, sich wechselseitig verstärkende, Effekte (z.B. Matthäus-Effekt) wirksam sind und zu der hohen Korrelation von Mehrfachengagement und leitender Position beitragen.

Der Vergleich der Determinanten der internen Hierarchisierung bei Männern und Frauen hat gezeigt, dass betreffend der weiteren askriptiven Merkmale (Alter und ethnische Herkunft) keine bemerkenswerten Unterschiede bestehen. Bei den erworbenen Merkmalen wurde deutlich, dass für Frauen der Bildungsgrad und für Männer eine leitenden Funktion im Beruf den Zugang zu leitenden Positionen in der Freiwilligenarbeit begünstigt. Verheiratet zu sein wirkt sich bei beiden Geschlechtern auf die Position in der Freiwilligenarbeit aus, hat jedoch für Männer eine größere Bedeutung. Betreffend der Statusreproduktion in der Freiwilligenarbeit hat sich somit gezeigt, dass die Reproduktion von Prestige und Status der Erwerbsarbeit in der Freiwilligenarbeit vorwiegend bei den Männern zu beobachten ist.

Das „Paradox der Gestreßtheit“ (Bourdieu 2001b: 291) trifft auch auf Personen in ehrenamtlichen Leitungsfunktionen zu. Je höher die berufliche Wochenarbeitszeit von Freiwilligen ist, desto häufiger bekleiden sie auch Leitungspositionen in der Freiwilligenarbeit. Ein höherer beruflicher Status und eine hohe berufliche Auslastung führen anscheinend dazu, sich ehrenamtlich um möglicherweise weniger zeitintensive Leitungstätigkeiten zu bemühen, als sich für zeitintensiver Kernaufgaben (z.B. Sanitäter bei Rettungsdiensten) zur Verfügung zu stellen.

$\mathrm{Da}$ vor dem Hintergrund der feldspezifischen Bedeutung der Kapitalien (Bourdieu 1977, 1982) anzunehmen ist, dass die vorangegangenen Ergebnisse und Thesen nicht auf alle Engagementbereiche in gleichem Maße zutreffen, bedarf es weiterer feldspezifischer Analysen. D.h. bevor aus den Ergebnissen allgemeine Schlüsse zu den Mechanismen und Prozessen sozialer Schließung und Hierarchisierung gezogen werden, werden vier der zentralen Engagementbereiche im nächsten Kapitel im Detail analysiert.

\section{(III) 4.4 Multivariate Analysen zu ausgewählten Feldern der Freiwilligenarbeit}

Das vierte und abschließende Analysekapitel widmet sich den feldspezifischen Determinanten und Ausprägungen der soziale Schließung und Hierarchisierung in der Freiwilligenarbeit. Folgende zwei Fragen sollen dabei beantwortet werden. (1) 
Welche askriptive wie erworbene soziale Merkmale beeinflussen den Zugang zur Freiwilligenarbeit in den ausgewählten gesellschaftlichen Feldern (Soziales; Religion; Sport; Rettungsdienste)? (2) Mit welchen askriptive wie erworbene sozialen Merkmalen und Kapitalien korrespondiert der Zugang zu den unterschiedlichen Aufgabenbereichen und hierarchischen Positionen in den jeweiligen Feldern? Einleitend wird die Auswahl der vier Felder begründet und anschließend die Über- und Unterrepräsentanz der sozialen Merkmalsgruppen in diesen Feldern analysiert und grafisch dargestellt. Der bivariaten Gegenüberstellung folgen die multivarianten Analysen (logistischen Regressionsmodelle) zu den feldspezifischen Determinanten des Zugangs. Abschließend werden die Regeln des Zugangs zu den unterschiedlichen hierarchischen Positionen in den vier Feldern mittels Multipler Korrespondenzanalysen untersucht.

\section{(III) 4.4.1. Ausgewählte Bereiche für die feldspezifischen Analysen}

Folgende Felder wurden zur weiteren Analyse ausgewählt: Soziales und Gesundheit; Kirche und Religion; Sport und Bewegung; Katastrophenhilfs- und Rettungsdienste (siehe Tabelle 30). Die Auswahl der Bereiche aus der Mikrozensuserhebung erfolgt auf Basis (1) der Bedeutung im österreichischen Freiwilligenbzw. Nonprofit-Sektor (vgl. BMASK 2009: 52), (2) der Geschlechterverteilung, und (3) der Zuordnung zu Eigenleistungs- und Fremdleistungsorganisationen bzw. zu binnen- und außenorientierten Vereinigungen (vgl. Braun 2007). Die Freiwilligen in den vier ausgewählten Bereichen, repräsentieren knapp 68\% der befragten Freiwilligen ( $\mathrm{N}=4.814$ ) der Mikrozensus-Zusatzerhebung (2006).

Tabelle 30: Tätigkeitsbereiche der Freiwilligenarbeit

\begin{tabular}{|l|l|l|l|l|}
\hline Bereich & $\begin{array}{l}\text { Beispiele für } \\
\text { Organisationen } \\
\text { bzw. Tätigkeiten }\end{array}$ & $\begin{array}{l}\text { Größe des } \\
\text { Freiwilligen- } \\
\text { sektors } \\
\text { (hochgerechnet) }\end{array}$ & $\begin{array}{l}\text { Geschlechter- } \\
\text { verteilung }\end{array}$ & $\begin{array}{l}\text { Orientierung } \\
\text { der Leistungser- } \\
\text { stellung }\end{array}$ \\
\hline $\begin{array}{l}\text { Soziales und } \\
\text { Gesundheit }\end{array}$ & $\begin{array}{l}\text { Sozial- bzw. } \\
\text { Hilfsorgani- } \\
\text { sationen, } \\
\text { Jugendzentren } \\
\text { bzw. -gruppen, } \\
\text { Seniorenorgani- } \\
\text { sationen, } \\
\text { Selbsthilfe- } \\
\text { gruppen, } \\
\text { Besuchs- oder } \\
\text { Begleitdienste }\end{array}$ & $\begin{array}{l}\sim 228.000 \\
\text { Freiwillige }\end{array}$ & $\begin{array}{l}\text { Frauen }> \\
\text { Männer } \\
(\sim 55 \%\end{array}$ & $\begin{array}{l}\text { vorwiegend } \\
\text { Fremdleistungs- } \\
\text { Frganisationen }) \\
\text { (außenorientiert) }\end{array}$ \\
\hline
\end{tabular}




\begin{tabular}{|c|c|c|c|c|}
\hline $\begin{array}{l}\text { Kirche und } \\
\text { Religion }\end{array}$ & $\begin{array}{l}\text { Pfarrgemein- } \\
\text { deräte, } \\
\text { Gremien von } \\
\text { Religionsgemein- } \\
\text { schaften, religiö- } \\
\text { se Kinder- oder } \\
\text { Jugendgruppen } \\
\text { bzw. Frauen und } \\
\text { Männerbewe- } \\
\text { gungen }\end{array}$ & $\begin{array}{l}\sim 429.000 \\
\text { Freiwillige }\end{array}$ & $\begin{array}{l}\text { Frauen > } \\
\text { Männer } \\
(\sim 66 \% \\
\text { Frauen })\end{array}$ & $\begin{array}{l}\text { gemischt } \\
\text { Fremd- und } \\
\text { Eigenleistungs- } \\
\text { organisationen } \\
\text { (außen- und } \\
\text { innenorientiert) }\end{array}$ \\
\hline $\begin{array}{l}\text { Sport und } \\
\text { Bewegung }\end{array}$ & $\begin{array}{l}\text { Sport- oder } \\
\text { Turnvereine, } \\
\text { Fachverbände } \\
\text { oder sonstige } \\
\text { Sportorgani- } \\
\text { sationen, } \\
\text { Bewegungs- } \\
\text { gruppen }\end{array}$ & $\begin{array}{l}\sim 475.000 \\
\text { Freiwillige }\end{array}$ & $\begin{array}{l}\text { Frauen < } \\
\text { Männer } \\
(\sim 69 \% \\
\text { Männer })\end{array}$ & $\begin{array}{l}\text { vorwiegend } \\
\text { Eigenleistungs- } \\
\text { organisationen } \\
\text { (innenorientiert) }\end{array}$ \\
\hline $\begin{array}{l}\text { Katastrophen- } \\
\text { hilfs- und } \\
\text { Rettungs- } \\
\text { dienste }\end{array}$ & $\begin{array}{l}\text { Freiwillige } \\
\text { Feuerwehren, } \\
\text { Rettungsdienste } \\
\text { (Rotes Kreuz, } \\
\text { Bergrettung), } \\
\text { humanitäre } \\
\text { Hilfsorgani- } \\
\text { sationen }\end{array}$ & $\begin{array}{l}\sim 413.000 \\
\text { Freiwillige }\end{array}$ & $\begin{array}{l}\text { Frauen < } \\
\text { Männer } \\
(\sim 83 \% \\
\text { Männer })\end{array}$ & $\begin{array}{l}\text { vorwiegend } \\
\text { Fremdleistungs- } \\
\text { organisationen } \\
\text { (außenorientiert) }\end{array}$ \\
\hline
\end{tabular}

Quelle: BMASK (2009: 212ff); More-Hollerweger/Sprajcer (2009).

(III) 4.4.2. Bivariate Analysen zur Über- und Unterrepräsentanz der sozialen Merkmalsgruppen in den Feldern der Freiwilligenarbeit

Da Art und Ausmaß der Kapitalakkumulation (symbolisch, sozial, kulturell etc.) durch Freiwilliges Engagement in den verschiedenen Feldern unterschiedlich ausgeprägt sein dürfte, ist zu erwarten, dass sich auch die soziale Zusammensetzung der Freiwilligen in den Engagementbereichen unterscheidet. In einem ersten Schritt wird die Über- bzw. Unterrepräsentanz einzelner Merkmalsgruppen in den vier ausgewählten Feldern untersucht. Zu diesem Zweck wird die Merkmalsverteilung in den Feldern mit der Merkmalsverteilung in der Österreichischen Gesamtbevölkerung ( $\geq 15$ Jahren) verglichen und die jeweiligen Abweichungen grafisch dargestellt ${ }^{74}$. Ergänzend werden Ergebnisse zur Über- und

74 Es werden hier im Sinne der Konsistenz die ungewichteten Daten des Mikrozensus 2006, 4. Quartal als Ausgangspunkt herangezogen. Die Anteile (Prozentwerte) weichen 
Unterrepräsentanz sozialer Gruppen in der Schweizer Freiwilligenarbeit angeführt (Stadelmann-Steffen et al. 2010: 170ff). Für Deutschland liegen leider keine derartigen Vergleichsergebnisse vor.

\section{Geschlecht}

Das Geschlechterverhältnis ist in den vier ausgewählten Feldern höchst unterschiedlich ausgeprägt. Die Verteilung im Feld des Sozialen und der Gesundheit (kurz: Soziales) entspricht nahezu der Verteilung in der österreichischen Gesamtbevölkerung. Frauen sind in diesem Feld in der Freiwilligenarbeit zu rund $+3 \%$ überrepräsentiert. Anders im Feld der Kirche und Religion (kurz: Religion). Hier sind Frauen mit $+14 \%$ deutlich überrepräsentiert. Im Feld des Sports und der Bewegung (kurz: Sport) und im Feld der Katastrophenhilfs- und Rettungsdienste (kurz: Katastrophenhilfe) kehrt sich die Verteilung deutlich um. Im Sport sind unter den Freiwilligen Männer mit $+22 \%$ deutlich überrepräsentiert. In der Katastrophenhilfe liegt der Männeranteil sogar $+36 \%$ über dem Anteil in der Bevölkerung.

Abbildung 19: Über- und Unterrepräsentation nach Geschlechtern in den Feldern

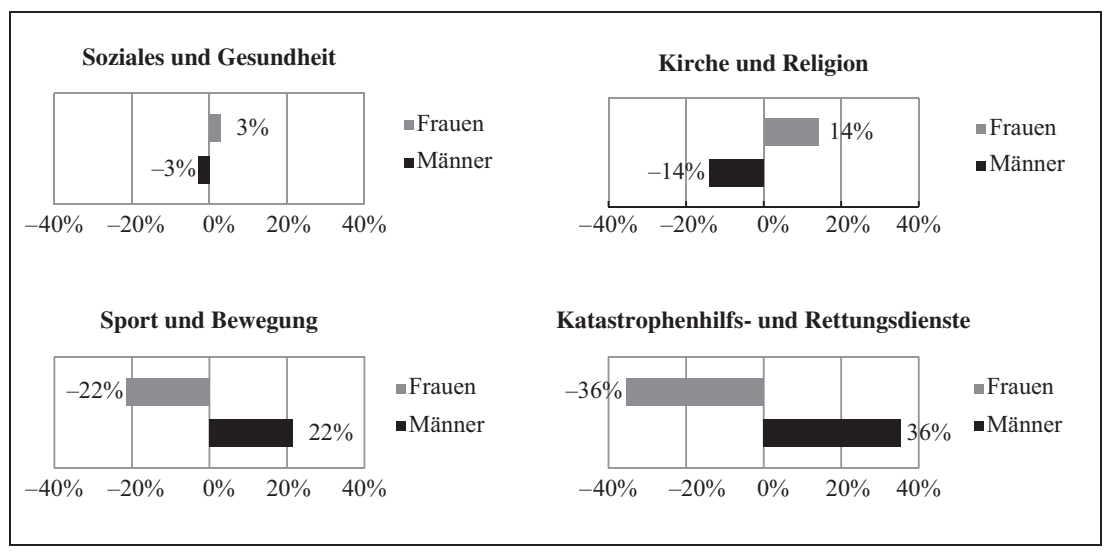

Quelle: Mikrozensus-Zusatzerhebung 2006/Q4; eigene Berechnungen.

daher geringfügig von anderen Publikationen der amtlichen Bevölkerungsstatistik, die überwiegend gewichtete Ergebnisse veröffentlichen, ab. Die Darstellungsform orientiert sich dabei an jener von Stadelmann-Steffen et al. (2010: 170ff) zur Analyse der brückenbildenden Funktion von Freiwilligenorganisationen im Rahmen des Schweizer Freiwilligenmonitors. 
Die Ergebnisse decken sich in ihrer Tendenz mit jenen aus der Schweiz aus dem Jahr 2010 (Stadelmann-Steffen et al. 2010: 172). Frauen sind dort in Sportvereinen mit -16\% etwas weniger stark unterrepräsentiert als in Österreich. In sozialen und karitativen Vereinen sind Frauen hingegegen mit $+12 \%$ deutlicher als in Österreich überrepräsentiert. Mit $+14 \%$ decken sich die Ergebnisse in beiden Ländern zur Überrepräsentanz von Frauen in kirchlichen Organisationen. In der Schweiz ist die Feuerwehr in den meisten Kantonen als Pflichtfeuerwehr ${ }^{75}$ organisiert und daher fehlt im Schweizer Freiwilligenmonitor ein Bereich, der in Umfang und Struktur der Freiwilligenarbeit im Feld der Katastrophenhilfs- und Rettungsdienste in Österreich entspricht.

\section{Alter}

Bezüglich der Über- und Unterrepräsentation der Altersgruppen gibt es zwischen den einzelnen Feldern der Freiwilligenarbeit erhebliche Unterschiede. Im Feld des Sozialen (-8\%) und im Feld der Religion (-9\%) ist die Gruppe der 15- bis 29-Jährigen deutlich unterrepräsentiert wohingegen im Feld des Sports $(-14 \%)$ und im Feld der Katastrophenhilfe (-13\%) die Gruppe der über 65-Jährigen deutlich unterrepräsentiert ist. Betreffend der Altersverteilung zeigt sich in den Schweizer Vereinen ein teilweise differentes Bild. Im Sport sind die über 65-Jährigen mit -11\% unterrepräsentiert, jedoch im Gegensatz zu Österreich in den sozialen und karitativen Vereinen $(+11 \%)$ sowie in den kirchlichen Organisationen (+8\%) deutlich überrepräsentiert (vgl. StadelmannSteffen et al. 2010: 175). Die Unterschiede zwischen den Feldern sind zum Teil auch dadurch erklärbar, dass zumindest in Österreich in bestimmten Bereichen der Katastrophenhilfe und der Rettungsdienste Altersgrenzen für bestimmte Tätigkeitsbereich (Rettungsfahrer, Bedienten bestimmter Geräte, etc.) existieren. Die Frage die sich diesbezüglich stellt ist, ob es den Organisationen künftig gelingen wird, für ältere Personen neue passende Tätigkeitsbereiche in den eigenen Organisationen oder aber auch in anderen Bereichen der Freiwilligenarbeit zu finden.

75 vgl. Schweizerischer Feuerwehrverband (http://www.swissfire.ch/ - abgerufen am 28.5.2014). 
Abbildung 20: Über- und Unterrepräsentation nach Altersgruppen in den Feldern

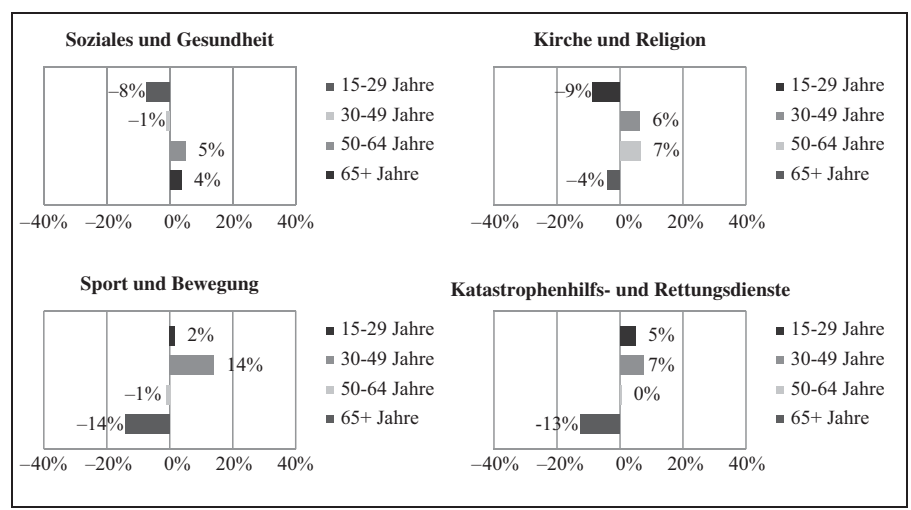

Quelle: Mikrozensus-Zusatzerhebung 2006/Q4; eigene Berechnung.

In diesem Zusammenhang sei auf die Arbeiten von Brudney/Meijs (2009) verwiesen, die Freiwillige als eine Art „erneuerbare“ Ressource auffassen. Freiwillige sollen demnach auf solche Art und Weise "gemanagt" und insbesondere „verabschiedet" werden, dass sie aufgrund positiver Erfahrungen für andere Freiwilligenorganisationen sowie Tätigkeits- und Engagementbereiche wieder zur Verfügung stehen.

\section{Geburtsland und Staatsbürgerschaft}

Personen, die nicht in Österreich geboren sind, sind in allen vier Feldern unterrepräsentiert. Mit rund $-5 \%$ ist dies im Feld des Sozialen, verglichen mit dem Feld der Religion und dem Feld des Sports mit jeweils -8\% und dem Feld der Katastrophenhilfe mit -10\%, am wenigsten der Fall.

Abbildung 21: Über- und Unterrepräsentation nach Geburtsland in den Feldern

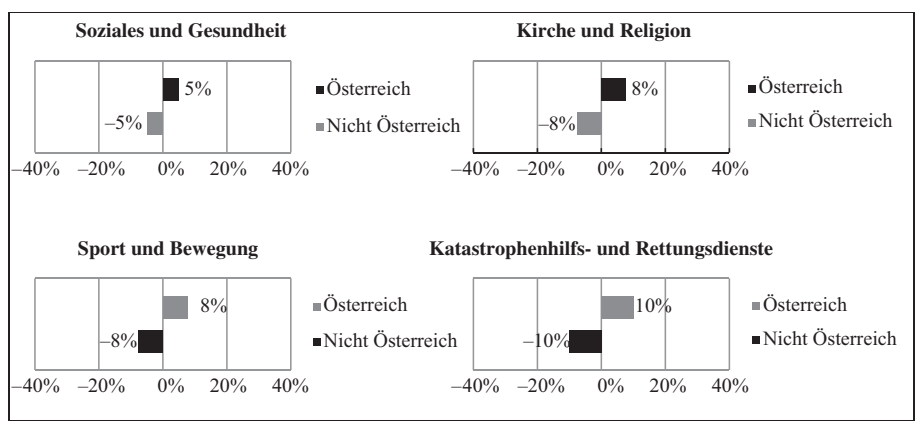

Quelle: Mikrozensus-Zusatzerhebung 2006/Q4; eigene Berechnung. 
Bei der Staatsbürgerschaft fällt die Unterrepräsentanz von Nicht-Österreichern in der Freiwilligenarbeit etwas geringer als beim Geburtsland aus. Dies liegt u.a. daran, dass der Anteil an Personen ohne österreichische Staatsbürgerschaft $(7,1 \%)^{76}$ in der Wohnbevölkerung geringer ist als der Anteil an Personen, die nicht in Österreich geboren (12,9\%) sind. Infolge sind auch die möglichen Abweichungen geringer.

Abbildung 22: Über- und Unterrepräsentation nach Staatsbürgerschaft in den Feldern

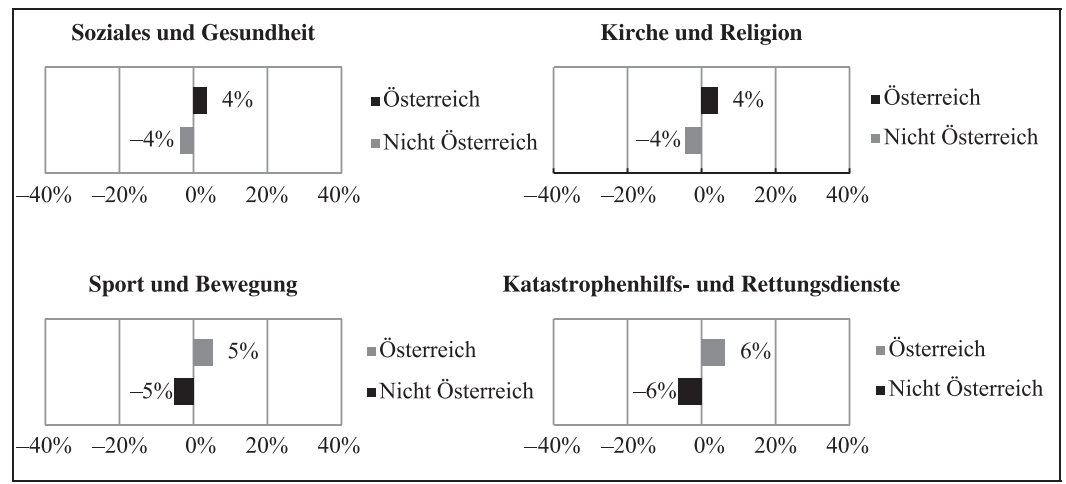

Quelle: Mikrozensus-Zusatzerhebung 2006/Q4; eigene Berechnung.

Gerade aber sind vor dem Hintergrund der Ergebnisse in Abbildung 22 die nun bereits gesetzlich verankerten Einbürgerungserleichterung durch den Nachweis einer dreijährigen Freiwilligentätigkeit, wie z.B. bei einer der Katastrophenhilfsund Rettungsdiensten (Feuerwehr, Rotes Kreuz, etc.), äußerst kritisch zu betrachten. Der Anteil von Personen ohne österreichische Staatsbürgerschaft lag 2006 bei den Katastrophenhilfs- und Rettungsdiensten bei lediglich 0,7\%. Auch bei der Freiwilligenarbeit im Feld des Sports, der Religion und des Sozialen sind Personen ohne österreichische Staatsbürgerschaft deutlich unterrepräsentiert. In der Schweiz sind aufgrund des höheren Anteils an Personen ohne Schweizer Staatsbürgschaft ${ }^{77}$ an der Wohnbevölkerung die Abweichungen etwas stärker ausgeprägt, deuten jedoch in dieselbe Richtung (Stadelmann-Steffen et al. 2010: 175).

76 Laut Statistik Austria lag der Anteil an Personen ohne österreichische Staatsbürgerschaft an der österreichischen Wohnbevölkerung am 1.1.2007 bei 14,7\%) (Quelle: http:// www.statistik.gv.at)

772010 lag der Anteil an Personen ohne Schweizer Staatsbürgerschaft bei 22\% der Wohnbevölkerung (Quelle: Statistik Schweiz: http://www.bfs.admin.ch/bfs/portal/ de/index/themen/01/07/blank/key/01/01.html) 


\section{Bildungsschicht}

Der Bildungsgrad als Determinante für Freiwilliges Engagement ist unbestritten, der Einfluss jedoch in den ausgewählten Feldern der Freiwilligenarbeit unterschiedlich ausgestaltet. Im Feld des Sozialen (-14\%) und der Religion (-10\%) sind sowohl Personen mit höchsten Pflichtschulabschluss als auch Personen mit Lehrabschluss deutlich unterrepräsentiert. Überrepräsentiert sind hingegen im Feld des Sozialen primär Absolventinnen und Absolventen tertiärer Ausbildungen (Kolleg, Uni-LG, hochschulverwandte Lehranstalten, Fachhochschulen und Universitäten) mit zusammengenommen $+14 \%$. Im Feld der Religion ist diese Gruppe mit $+9 \%$ ebenfalls überrepräsentiert. Die Ergebnisse zum Feld des Sozialen decken sich in ihrer Richtung mit den Ergebnissen aus der Schweiz. Personen mit einem tertiären Bildungsabschluss sind in diesem Bereich $\mathrm{zu}+17 \%$ überrepräsentiert (Stadelmann-Steffen et al. 2010: 176). Bei den kirchlichen Organisationen hingegen gibt es in der Schweiz nur geringfügige Abweichungen von der Bevölkerungsverteilung (ebd.). Dies könnte u.a. auf die unterschiedliche Ausgestaltung des religiösen Feldes in der Schweiz, z.B. betreffend der Anzahl an Freikirchen, und Österreich zurückzuführen sein.

Abbildung 23: Über- und Unterrepräsentation nach Bildungsschicht in den Feldern

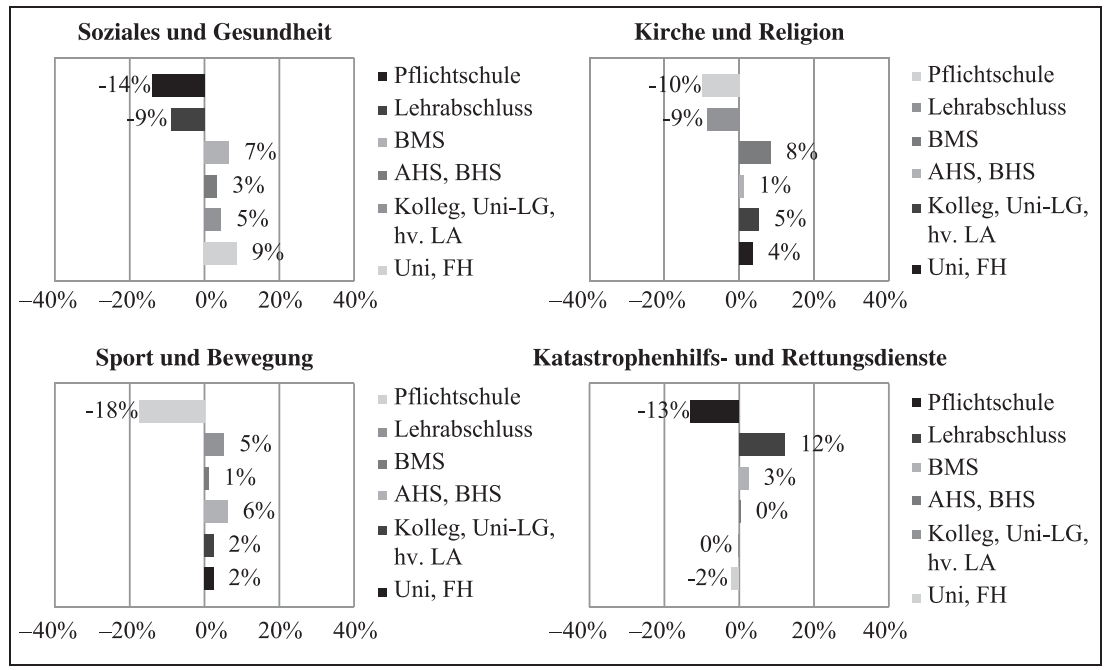

Quelle: Mikrozensus-Zusatzerhebung 2006/Q4; eigene Berechnung.

Im Feld des Sports ist vor allem die unterste Bildungsschichte mit -18\% erheblich unterrepräsentiert. Damit spiegelt sich die vergleichsweise geringe Sportbeteiligung 
von bildungsfernen Schichten auch bei der Freiwilligenarbeit im Sport wieder (vgl. Nagel 2003b). In der Schweiz fällt die Abweichung vom Durchschnitt mit -6\% deutlich geringer aus (Stadelmann-Steffen et al. 2010: 176). Die Katastrophenhilfe weist ebenfalls eine Unterrepräsentanz hinsichtlich Freiwilliger mit dem niedrigsten Bildungsabschluss von -13\% auf. Mit 12\% deutlich überrepräsentiert sind Personen mit Lehrabschluss. Dies erklärt sich zum Teil aus den z.B. bei der Freiwilligen Feuerwehr benötigten handwerklichen Kompetenzen und der größeren Bedeutung dieses Engagementbereichs in ländlichen Regionen. Universitäts- und FachhochschulabsolventInnen sind im Feld der Katastrophenhilfe mit-2\% unterrepräsentiert.

\section{Erwerbstätigkeit und leitenden Funktion im Beruf}

Wie sich in den vorangegangen Analysen zum Einfluss der Erwerbstätigkeit auf den allgemeinen Zugang zu Freiwilligenarbeit gezeigt hat (vgl. Kapitel (III) 2.1. und (III) 2.2.), gibt es zwar bivariat einen positiven Zusammenhang zwischen Erwerbsarbeit und Freiwilligenarbeit, in den multivariaten Modellen konnte dieser jedoch nicht bestätigt werden. Betreffend den Zugang zu den einzelnen Feldern der Freiwilligenarbeit scheint der Einfluss der Erwerbsarbeit jedoch stark zu variieren.

Abbildung 24: Über- und Unterrepräsentation nach Erwerbstätigkeit in den Feldern

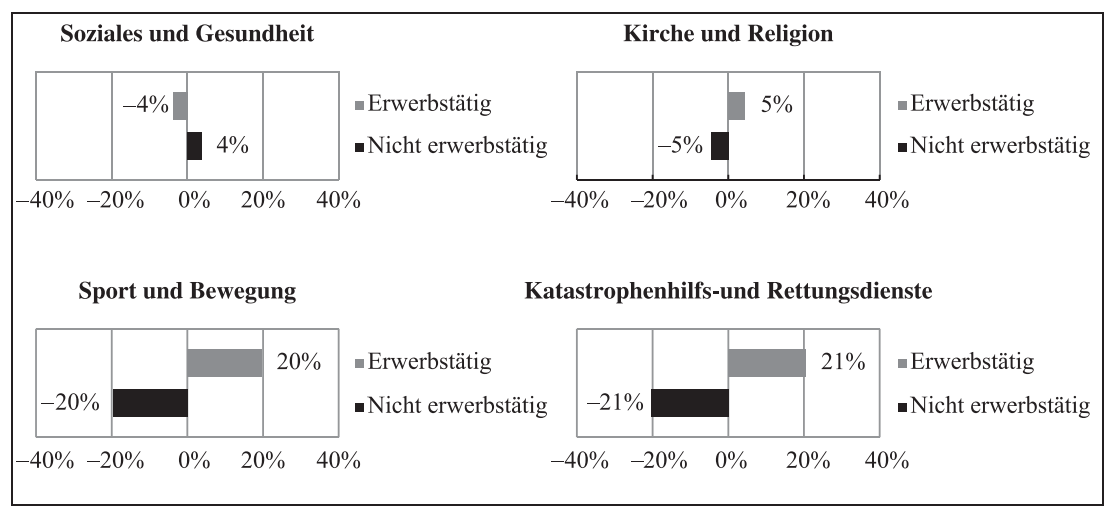

Quelle: Mikrozensus-Zusatzerhebung 2006/Q4; eigene Berechnung.

Im Feld des Sozialen und im Feld der Religion sind nicht-erwerbstätige Personen geringfügig unterrepräsentiert, im Sozialen um -4\% und in der Religion um -5\%. Ein anderes Bild zeigt sich im Feld des Sports und im Feld der Katastrophenhilfe. In diesen Bereichen der Freiwilligenarbeit sind nicht-erwerbstätige Personen mit rund $-20 \%$ stark unterrepräsentiert. Hier gilt es in den multivariaten 
Regressionsanalysen (siehe Kapitel (III) 4.4.3.) zu überprüfen, ob diese Ergebnisse durch den hohen Männeranteil in diesen beiden Feldern moderiert wird. Stadelmann-Steffen et al. (2010) bieten zu beruflichen Merkmalen keine Ergebnisse für die Schweiz.

Abbildung 25: Über- und Unterrepräsentation nach Funktion im Beruf in den Feldern

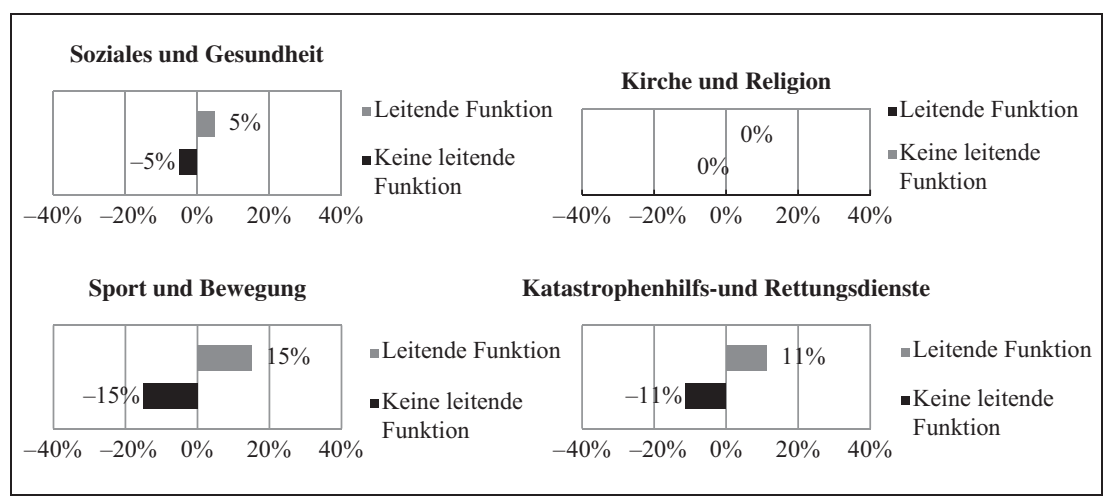

Quelle: Mikrozensus-Zusatzerhebung 2006/Q4; eigene Berechnung.

Sofern man erwerbstätig ist, stellt sich auch die Frage, ob die Tätigkeit mit einer Leitungsfunktion und damit auch mit einem bestimmten Mindestmaß an beruflichem Status verbunden ist. Im Feld des Sozialen sind Personen mit Leitungsfunktion im Beruf mit $+5 \%$ überrepräsentiert, wohingegen die Verteilung im Feld der Religion diesbezüglich der Verteilung in der Bevölkerung entspricht. In den „männerdominierten“ Feldern Sport und Katastrophenhilfe sind erwartungsgemäß Personen mit Leitungsfunktion im Beruf, mit $+15 \%$ im Sport und mit $+11 \%$ in der Katastrophenhilfe überrepräsentiert. Auch in diesem Fall stellt sich die Frage nach dem moderierenden Einfluss des Geschlechts.

\section{Wohnrechtsverhältnis}

Zum Abschluss stellt sich noch die Frage nach dem Einfluss des ökonomischen Kapitals auf die Freiwilligenarbeit in den ausgewählten Feldern. Trotz der erheblichen Unterschiede zwischen den Feldern bei den sozialen Merkmalen Geschlecht, Alter, Bildungsgrad und Erwerbstätigkeit, decken sich die Einflussrichtungen des Zusammenhangs von Wohneigentum und Freiwilligenarbeit in den ausgewählten Feldern.

Personen, die in Wohneigentum leben, sind im Feld des Sozialen mit 11\%, im Feld des Sports mit $+17 \%$, im Feld der Religion mit $+21 \%$ und schließlich 
im Feld der Katastrophenhilfe mit $+22 \%$ überrepräsentiert. Trotz fehlender Daten zum Einkommen in den Mikrozensusdaten 2006 sei kurz auf die Daten des Schweizer Freiwilligenmonitors verwiesen. In der Schweiz sind Personen mit einem monatlichen Einkommen unter 5.000 CHF in sozialen und karitativen Vereinen mit $-11 \%$ und in Sportvereinen mit $-12 \%$ unterrepräsentiert.

Abbildung 26: Über- und Unterrepräsentation nach Wohnrechtsverhältnis in den Feldern

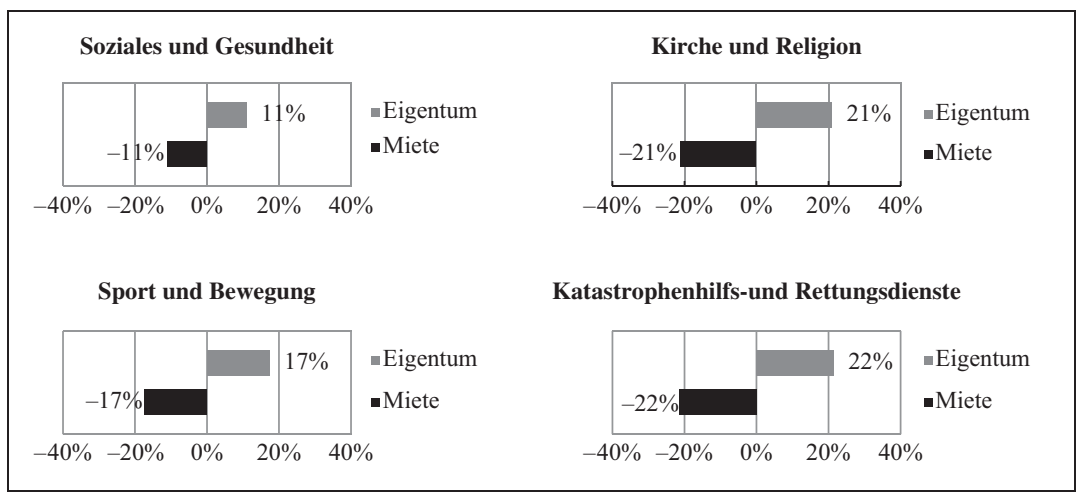

Quelle: Mikrozensus-Zusatzerhebung 2006/Q4; eigene Berechnung.

In kirchlichen Organisationen hingegen sind Personen mit einem Einkommen über 11.000 CHF unterrepräsentiert (vgl. Stadelmann-Steffen et al. 2010: 178). Diese Ergebnisse machen nochmals die Bedeutung von Wohneigentum, interpretiert als ökonomisches Kapital in Form von Vermögen oder aber als ein Anzeichen einer ausgeprägten Verwurzelung am Wohnort (vgl. Rotolo et al. 2010), für Freiwilliges Engagement deutlich.

In einem nächsten Schritt erfolgt die multivariate Überprüfung der Ergebnisse zum Einfluss der sozialen Merkmale auf den Zugang zur Freiwilligenarbeit in den ausgewählten Feldern.

\section{(III) 4.4.3. Multivariate Regressionsanalysen zur sozialen Schließung in den Feldern}

Die bivariaten Ergebnisse zum Zugang von Männern und Frauen zu den ausgewählten Feldern bestätigt sich in der Grundtendenz auch in den multivariaten Regressionsanalysen (siehe Tabelle 31). Im Feld des Sozialen haben Frauen eine geringfügig höhere Chance für den Zugang (odds ratio: 1,150). Im Feld der Religion ist hingegen die Chance von Frauen gegenüber Männern um 90\% 
erhöht (odds ratio: $1,900^{* * *}$ ). Im Feld des Sports sowie der Katastrophenhilfe haben Frauen eine geringere Chance als Männer: Im Sport um 61,7\% (odds ratio: $0,393^{* * *}$ ) und in der Katastrophenhilfe um $83,8 \%$ (odds ratio: $0,162^{* * *}$ ).

Tabelle 31: Logistische Regression - Determinanten der formelle Freiwilligenarbeit in den vier ausgewählten Feldern

\begin{tabular}{|c|c|c|c|c|c|}
\hline & \multicolumn{5}{|c|}{ Abhängige Variable: Formelle Freiwilligenarbeit } \\
\hline & $\begin{array}{l}\text { Formelle } \\
\text { Freiwilli- } \\
\text { genarbeit }\end{array}$ & $\begin{array}{l}\text { Soziales } \\
\text { und } \\
\text { Gesund- } \\
\text { heit }\end{array}$ & $\begin{array}{l}\text { Kirche } \\
\text { und } \\
\text { Religion }\end{array}$ & $\begin{array}{l}\text { Sport und } \\
\text { Bewegung }\end{array}$ & $\begin{array}{l}\text { Katastro- } \\
\text { phenhilfs- } \\
\text { und Rettungs- } \\
\text { dienste }\end{array}$ \\
\hline & $\operatorname{Exp}(B)$ & $\operatorname{Exp}(B)$ & $\operatorname{Exp}(B)$ & $\operatorname{Exp}(B)$ & $\operatorname{Exp}(B)$ \\
\hline Frauen (Ref. Männer) & $0,570^{* * *}$ & 1,150 & $1,900^{* * *}$ & $0,393^{* * *}$ & $0,162^{* * *}$ \\
\hline Alter & $0,990^{* * *}$ & $1,015^{* * *}$ & $1,006^{*}$ & $0,982^{* * *}$ & $0,987^{* * *}$ \\
\hline $\begin{array}{l}\text { Geburtsland } \\
\text { (Ref. Österreich) }\end{array}$ & $0,581^{* * *}$ & 0,822 & $0,569^{* * *}$ & $0,715^{*}$ & $0,467^{* * *}$ \\
\hline Bildungsgrad ( 1 bis 6 ) & $1,217^{* * *}$ & $1,305^{* * *}$ & $1,200^{* * *}$ & $1,102^{* * *}$ & $0,928^{* *}$ \\
\hline $\begin{array}{l}\text { Erwerbstätig (Ref. } \\
\text { Erwerbslos, Arbeitslos, } \\
\text { in Pension, Präsenz- } \\
\text { oder Zivildiener) }\end{array}$ & 1,010 & $0,571^{* * *}$ & 0,915 & 1,146 & $1,430^{* * *}$ \\
\hline $\begin{array}{l}\text { Leitenden Funktion } \\
\text { im Beruf (Ref. keine } \\
\text { leitenden Funktion) }\end{array}$ & $1,145^{* *}$ & $1,307^{*}$ & $0,798^{*}$ & $1,367^{* * *}$ & 0,979 \\
\hline $\begin{array}{l}\text { Wohnrechtsverhältnis } \\
\text { Eigentum (Ref. Miete, } \\
\text { Untermiete, sonstiges) }\end{array}$ & $1,421^{* * *}$ & 0,942 & $1,394^{* * *}$ & $1,225^{* *}$ & $1,269^{* *}$ \\
\hline $\begin{array}{l}\text { Verheiratet (Ref. Ledig, } \\
\text { Geschieden, Verwitwet) }\end{array}$ & $1,390^{* * *}$ & 1,035 & $1,909^{* * *}$ & $1,209^{*}$ & 1,048 \\
\hline Bevölkerungsdichte & $0,740^{* * *}$ & 0,927 & $0,809^{* * *}$ & $0,828^{* * *}$ & $0,541^{* * *}$ \\
\hline Konstante & $2,911^{* * *}$ &, $019^{* * *}$ & $0,024^{* * *}$ & 0,944 & $8,755^{* * *}$ \\
\hline $\mathrm{N}$ & 11.657 & 11.657 & 11.657 & 11.657 & 11.657 \\
\hline $\mathrm{Chi}^{2} / \mathrm{df}$ & $936 / 9$ & $158 / 9$ & $347 / 9$ & $454 / 9$ & $922 / 9$ \\
\hline-2 Log-Likelihood & $14.868,17$ & 4541,160 & 6821,242 & 7218,465 & 5685,946 \\
\hline $\begin{array}{l}\text { Pseudo R }{ }^{2} \\
\text { (Nagelkerke’s) }\end{array}$ & 0,104 & 0,041 & 0,064 & 0,079 & 0,176 \\
\hline
\end{tabular}

Quelle: Mikrozensus-Zusatzerhebung 2006/Q4; eigene Berechnung; $\left(p^{*}<0.05 ; p^{* *}<0.01 ; p^{* * *}<0.001\right)$. 
Ein höheres Alter wirkt sich tendenziell im Feld des Sozialen (odds ratio: $1,015^{* * *}$ ) und der Religion (odds ratio: $1,006^{*}$ ) positiv auf ein Engagement aus, im Feld des Sports (odds ratio: 0,982 $2^{* * *}$ ) und der Katastrophenhilfe (odds ratio: $\left.0,987^{* * *}\right)$ hingegen negativ.

Die bivariaten feldspezifischen Ergebnisse zum Einfluss des Geburtslands bestätigen sich im Großen und Ganzen auch multivariat. Lediglich im Feld des Sozialen ist die ethnische Herkunft, gemessen am Geburtsland, keine signifikante Determinante (odds ratio: 0,822). Im Feld der Kirche (odds ratio: 0,569 $9^{* * *}$ ), des Sports (odds ratio: $0,715^{*}$ ) und des Katastrophenhilfe (odds ratio:0,467 ${ }^{* *}$ ) finden nicht in Österreich geborene Personen, auch unter Beachtung des Geschlechts, des Alters, und des Bildungsgrads erschwerten Zugang zur Freiwilligenarbeit.

Dass sich Investitionen in den Bildungsgrad der Bevölkerung grundsätzlich positiv auf die Beteiligung am Freiwilligen Engagement auswirken, zeigen neben den Ergebnissen zur gesamten formellen Freiwilligenarbeit auch die feldspezifischen Analysen. Eine Ausnahme unter den ausgewählten Feldern bildet jedoch die Katastrophenhilfe (odds ratio: $0,928^{* *}$ ). Hier wirkt sich ein höherer und insbesondere ein tertiärer Bildungsgrad negativ auf die Beteiligung aus. $\mathrm{Ob}$ es in Folge einer weiteren Bildungsexpansion, d.h. konkret einer Tertiärisierung, langfristig in diesen Bereichen zu einer notwendigen Professionalisierung im Sinne eines Wandels zum Hauptamt kommen wird und wer ggf. dafür die Kosten trägt, bleibt abzuwarten.

Der bivariat beobachtbare Einfluss der Erwerbstätigkeit auf den Zugang zum Freiwilligen Engagement bestätigt sich nicht in allen der ausgewählten Felder. Im Feld des Sozialen haben Erwerbstätige eine um 42,9\% reduzierte Chance des Zugangs zu freiwilligen Tätigkeiten. Im Feld der Religion gibt, vorausschlich moderiert durch das Geschlecht, einen negativen jedoch keinen signifikanten Einfluss des Erwerbsstatus (odds ratio: 0,915). Im Feld des Sports dürfte ebenfalls der Einfluss der Erwerbstätigkeit durch den hohen Männeranteil moderiert werden und ist positiv, jedoch nicht signifikant (odds ratio: 1,146). Hingegen ist im Feld der Katastrophenhilfe der Einfluss der Erwerbstätigkeit trotz der Kontrolle für das Geschlecht und das Alter mit einer odds ratio von $1,430^{* * *}$ hoch signifikant. Die weitere Spezifizierung der beruflichen Funktion zeigt, dass eine leitende Position in der Erwerbsarbeit einen positiven Einfluss auf den Zugang zum Feld des Sozialen (odds ratio: 1,307 ${ }^{*}$ ) und zum Feld des Sports (odds ratio: $\left.1,367^{* * *}\right)$ hat. Einen negativen Einfluss hat eine leitende Funktion im Beruf für den Zugang zur Freiwilligenarbeit in der Religion (odds ratio: 0,797*). Auf den Zugang zur Freiwilligenarbeit im Feld der Katastrophenhilfe hingegen hat die 
berufliche Position neben der der Erwerbstätigkeit im Allgemeinen keinen zusätzlichen Einfluss mehr.

Der positive Einfluss von Wohneigentum auf den Zugang zur Freiwilligenarbeit bestätigt sich, mit Ausnahme des Feldes des Sozialen, für die ausgewählten Felder. Wohneigentum erhöht die Chance für Freiwilliges Engagement im Feld der Religion um 39,4\%, im Feld des Sports um 22,5\% und im Feld der Katastrophenhilfe um $26,9 \%$.

Gesamt betrachtet gelten die Zugangsvoraussetzungen, d.h. ein bestimmtes Maß an Kapitalausstattung, wie es für die Freiwilligenarbeit im Gesamten zu beobachten ist, mit jeweils kleinen Abweichungen und Besonderheiten auch in den ausgewählten Feldern der Freiwilligenarbeit. Das Feld des Sozialen scheint primär über kulturelles Kapital zu selektieren, das Geschlecht, das Geburtsland und die ökonomische Ausstattung dürften von nachgeordneter Bedeutung sein. Das Feld der Religion selektiert zentral über das Geschlecht, die ethnische Herkunft sowie das kulturelle wie ökonomische Kapital. Im Feld des Sports und des Katastrophenhilfe ist der Zugang vordergründig durch das Geschlecht, die ethnische Herkunft, das symbolische Kapital des Erwerbsfeldes sowie durch das ökonomische Kapital bestimmt.

Prozesse sozialer Schließung sind den empirischen Ergebnissen zur Folge in allen Feldern zu beobachten. Die sozialen Merkmale, die als Schließungskriterien fungieren, unterscheiden sich jedoch teils erheblich zwischen den Feldern des Engagements. Da die Daten nur auf Feldebene und nicht auf Organisationsebene erfasst wurden, sind diese Ergebnisse primär als Anregung für die einzelnen Organisationen gedacht, ihre impliziten wie expliziten $\mathrm{Zu}$ gangsregeln einer kritischen Reflexion zu unterziehen. Ganzen Engagementfeldern grundlegend ihr integratives Potential abzusprechen, kann und soll nicht behauptet werden. Auf politischer Ebenen hingegen sollten die Befunde dazu führen, die Erwartungen an die Freiwilligenarbeit sowie die damit verbundenen Kausalannahmen hinsichtlich der positiven Effekte zu überdenken und ihre Potentialeinschätzungen in Zukunft vermehrt an empirischen Evidenzen auszurichten.

\section{(III) 4.4.4. Multivariate Korrespondenzanalysen zur Hierarchisierung in den Feldern}

Wie bereits im Kapitel zu den statischen Analyseverfahren beschrieben, handelt es sich bei der multiplen Korrespondenzanalyse (kurz: MCA) um ein multivariates Verfahren zur grafischen Darstellung von mehrdimensionalen Kontingenztabellen (vgl. Greenacre 2007; Le Roux/Rouanet 2010). Es gibt bei diesem 
Verfahren keine „abhängigen“ und „unabhängigen“ Variablen. Die Analyse beschränkt sich in der vorliegenden Arbeit auf die Interpretation der Diagramme. Die nachfolgenden grafischen Darstellungen zu den Engagementbereichen (Abbildungen 27 bis 30) sind folgendermaßen zu lesen und zu interpretieren: Variablenausprägungen, die räumlich weiter von einander entfernt liegen, kommen seltener (unterdurchschnittlich) gemeinsam vor als jene, die vergleichsweise näher (überdurchschnittlich) liegen. Die Lage der Variablen sagt dabei jedoch nichts über deren absolute Häufigkeit aus.

\section{Variablenbeschreibungen}

Für die Analyse und Bestimmung der Stellung der freiwillig tätigen Personen in den jeweiligen Feldern der Freiwilligenarbeit wurden sechs Variablen auf Basis der vorangegangen Ergebnisse ausgewählt (siehe Tabelle 32). Die beiden Merkmale Geschlecht und Alter wurden zu einer neuen Variable kombiniert, die je Geschlecht vier Altersgruppen umfasst. Als weitere Variablen fungieren das Wohnrechtsverhältnis als Indikator für ökonomisches Kapital und der Bildungsgrad als institutionalisiertes kulturelles Kapital. Das symbolische Kapital des Erwerbsfeldes wird durch die berufliche Stellung abgebildet, und das symbolische Kapital im jeweiligen Feld der Freiwilligenarbeit durch die Funktion, bzw. Position in der Freiwilligenarbeit ${ }^{78}$. Die Tabelle 32 gibt auch über die Häufigkeit und die prozentuelle Verteilung der Variablen in den einzelnen Feldern Aufschluss.

78 Im Rahmen der Mikrozensusbefragung war es möglich, sofern man in einem Bereich (z.B. Sport) in mehreren Organisationen tätig ist, für jede Organisation die jeweilige Funktion anzugeben. Da Mehrfachantworten keine eindeutige Zuordnung der Personen zu einem Funktionsbereich (leitend, ausführend, ...) ermöglichen, wurde je Person und Engagementbereich nur die, für die erstgenannte Organisation angeführte Funktion, für die Auswertung herangezogen. Dieser Schritt ist auch damit zu begründen, dass angenommen werden kann, dass die erstgenannte Organisation auch die bedeutsamste für die Person darstellt. 
Tabelle 32: Variablen, Kategorien und deren Ausprägung (absolute und relative Häufigkeiten) in den Feldern

\begin{tabular}{|c|c|c|c|c|c|c|c|c|c|}
\hline \multirow[b]{2}{*}{ Variable } & \multirow[b]{2}{*}{ Kategorien } & \multicolumn{2}{|c|}{$\begin{array}{l}\text { Soziales und } \\
\text { Gesundheit }\end{array}$} & \multicolumn{2}{|c|}{$\begin{array}{l}\text { Kirche und } \\
\text { Religion }\end{array}$} & \multicolumn{2}{|c|}{$\begin{array}{l}\text { Sport und } \\
\text { Bewegung }\end{array}$} & \multicolumn{2}{|c|}{$\begin{array}{c}\text { Katastro- } \\
\text { phenhilfs- und } \\
\text { Rettungsdienste }\end{array}$} \\
\hline & & $\mathbf{n}$ & $\%$ & $\mathbf{n}$ & $\%$ & $\mathrm{n}$ & $\%$ & $\mathrm{n}$ & $\%$ \\
\hline \multirow[t]{8}{*}{$\begin{array}{l}\text { Geschlecht und } \\
\text { Alter }\end{array}$} & $\begin{array}{l}\text { männlich } \\
\text { 15-29 Jahre }\end{array}$ & 40 & 6,7 & 53 & 4,9 & 193 & 16,3 & 195 & 20,4 \\
\hline & $\begin{array}{l}\text { männlich } \\
\text { 30-49 Jahre }\end{array}$ & 86 & 14,5 & 131 & 12,2 & 413 & 34,8 & 367 & 38,5 \\
\hline & $\begin{array}{l}\text { männlich } \\
\text { 50-64 Jahre }\end{array}$ & 72 & 12,1 & 102 & 9,5 & 171 & 14,4 & 175 & 18,3 \\
\hline & $\begin{array}{l}\text { männlich } \\
65+\text { Jahre }\end{array}$ & 68 & 11,4 & 75 & 7,0 & 45 & 3,8 & 57 & 6,0 \\
\hline & $\begin{array}{l}\text { weiblich } \\
\text { 15-29 Jahre }\end{array}$ & 37 & 6,2 & 73 & 6,8 & 68 & 5,7 & 46 & 4,8 \\
\hline & $\begin{array}{l}\text { weiblich } \\
30-49 \text { Jahre }\end{array}$ & 134 & 22,5 & 346 & 32,2 & 205 & 17,3 & 67 & 7,0 \\
\hline & $\begin{array}{l}\text { weiblich } \\
50-64 \text { Jahre }\end{array}$ & 86 & 14,5 & 200 & 18,6 & 71 & 6,0 & 35 & 3,7 \\
\hline & $\begin{array}{l}\text { weiblich } \\
65+\text { Jahre }\end{array}$ & 72 & 12,1 & 94 & 8,8 & 21 & 1,8 & 12 & 1,3 \\
\hline \multirow{4}{*}{$\begin{array}{l}\text { Wohnrechts- } \\
\text { verhältnis }\end{array}$} & Hauseigentum & 390 & 65,5 & 820 & 76,4 & 870 & 73,3 & 768 & 80,5 \\
\hline & Miete & 132 & 22,2 & 142 & 13,2 & 217 & 18,3 & 123 & 12,9 \\
\hline & $\begin{array}{l}\text { sonstiges } \\
\text { Wohnrechts- } \\
\text { verhältnis }\end{array}$ & 13 & 2,2 & 24 & 2,2 & 11 & 0,9 & 13 & 1,4 \\
\hline & Wohneigentum & 60 & 10,1 & 88 & 8,2 & 89 & 7,5 & 50 & 5,2 \\
\hline \multirow[t]{6}{*}{ Bildungsgrad } & $\begin{array}{l}\text { höchstens } \\
\text { Pflichtschule }\end{array}$ & 87 & 14,6 & 202 & 18,8 & 133 & 11,2 & 150 & 15,7 \\
\hline & Lehrabschluss & 161 & 27,1 & 294 & 27,4 & 490 & 41,3 & 460 & 48,2 \\
\hline & $\begin{array}{l}\text { berufsbildende } \\
\text { mittlere Schule }\end{array}$ & 120 & 20,2 & 236 & 22,0 & 175 & 14,7 & 154 & 16,1 \\
\hline & $\begin{array}{l}\text { Matura } \\
\text { (AHS/BHS) }\end{array}$ & 95 & 16,0 & 148 & 13,8 & 223 & 18,8 & 124 & 13,0 \\
\hline & $\begin{array}{l}\text { Kolleg, Uni } \\
\text { Lehrgang, PädAk }\end{array}$ & 39 & 6,6 & 79 & 7,4 & 54 & 4,5 & 18 & 1,9 \\
\hline & Uni/FH & 93 & 15,6 & 115 & 10,7 & 112 & 9,4 & 48 & 5,0 \\
\hline Berufliche & Arbeiter & 32 & 5,4 & 88 & 8,2 & 193 & 16,3 & 257 & 26,9 \\
\hline \multirow[t]{5}{*}{ Stellung } & Angestellte & 171 & 28,7 & 387 & 36,0 & 502 & 42,3 & 305 & 32,0 \\
\hline & Erwerbslose & 281 & 47,2 & 419 & 39,0 & 284 & 23,9 & 219 & 23,0 \\
\hline & Führungskräfte & 47 & 7,9 & 63 & 5,9 & 115 & 9,7 & 59 & 6,2 \\
\hline & Landwirte & 12 & 2,0 & 66 & 6,1 & 13 & 1,1 & 69 & 7,2 \\
\hline & Selbständige & 52 & 8,7 & 51 & 4,7 & 80 & 6,7 & 45 & 4,7 \\
\hline \multirow[t]{4}{*}{$\begin{array}{l}\text { Funktion in der } \\
\text { Freiwilligenarbeit }\end{array}$} & $\begin{array}{l}\text { Administrative } \\
\text { Aufgaben }\end{array}$ & 184 & 30,9 & 318 & 29,6 & 399 & 33,6 & 164 & 17,2 \\
\hline & Kernaufgaben & 267 & 44,9 & 516 & 48,0 & 422 & 35,6 & 661 & 69,3 \\
\hline & $\begin{array}{l}\text { Leitenden } \\
\text { Funktion }\end{array}$ & 85 & 14,3 & 91 & 8,5 & 190 & 16,0 & 66 & 6,9 \\
\hline & sonstige Aufgaben & 59 & 9,9 & 149 & 13,9 & 176 & 14,8 & 63 & 6,6 \\
\hline Gesamt & & 595 & 100,0 & 1.074 & 100,0 & 1.187 & 100,0 & 955 & 100,0 \\
\hline
\end{tabular}

Quelle: Mikrozensus-Zusatzerhebung 2006/Q4; eigene Berechnung. 


\section{(III) 4.4.5. Positionen im Feld des Sozialen und der Gesundheit}

Laut Hochrechnung von Statistik Austria waren im Jahr 2006 im Feld des Sozialen und der Gesundheit rund 288.000 Freiwillige tätig, die wöchentlich rund 565.000 Arbeitsstunden geleistet haben (vgl. More-Hollerweger/Sprajcer 2009: 41). Das Feld des Sozialen und der Gesundheit umfasst u.a. Sozial- bzw. Hilfsorganisationen, Jugendzentren bzw. -gruppen, Seniorenorganisationen, Selbsthilfegruppen sowie Besuchs- oder Begleitdienste. In Österreich sind die größten Organisationen in diesem Feld u.a. die Caritas ${ }^{79}$, das Rote Kreuz ${ }^{80}$, das Österreichische Hilfswerk $^{81}$, die Diakonie ${ }^{82}$ und die Volkshilfe Österreich ${ }^{83}$ (anzunehmen sind Überschneidung mit dem Feld der Katastrophenhilfs- und Rettungsdienste sowie dem Feld der Kirche und Religion). Zur internen Strukturierung und Hierarchisierung der Freiwilligen gibt es von Seiten der Organisationen keine Angaben.

Wie die Analyse der Determinanten des Zugangs zum Feld des Sozialen und der Gesundheit gezeigt hat, ist vor allem die Höhe des Bildungsgrads, d.h. des kulturellen Kapitals entscheidend. Mit steigendem Bildungsgrad erhöht sich die Wahrscheinlichkeit für ein Freiwilliges Engagement in diesem Feld. Erwerbstätig zu sein ist hingegen hinderlich, außer man ist im Beruf in einer leitenden Position. Der negative Einfluss der Erwerbstätigkeit lässt sich zumindest zum Teil mit dem vergleichsweise hohen Anteil an Freiwilligen über 65 Jahre von rund 24\% erklären (siehe auch Tabelle 40). Zumindest betreffend den Zugang zur Freiwilligenarbeit im Feld des Sozialen und der Gesundheit zeigt sich unter den vier ausgewählten Feldern die loseste Kopplung mit dem Feld der Erwerbsarbeit. Wohneigentum spielt entgegen dessen positiven Einfluss auf die Freiwilligenarbeit im Gesamten, im Feld des Sozialen und der Gesundheit keine Rolle.

79 Caritas: 35.000 Freiwillige (Quelle: http://www.caritas.at/ueber-uns/zahlen-fakten/ abgerufen am 22.5.2014).

80 Rotes Kreuz: 56.000 Freiwillige (Quelle: http://www.roteskreuz.at/mitmachen/mitarbeit/freiwillige-mitarbeit/ - abgerufen am 22.5.2014).

81 Hilfswerk: 20.000 Freiwillige (Quelle: http://www.hilfswerk.at/jobs/freiwillige-undehrenamtliche-mitarbeit - abgerufen am 22.5.2014).

82 Diakonie: 2.000 Freiwillige (Quelle: http://www.diakonie.at/goto/de/diakonie/mitarbeit?type=ehrenamtlich - abgerufen am 22.5.2014).

83 Volkshilfe: 3. 000 Freiwillige (Quelle: https://www.volkshilfe.at/presse-archiv?iD=82 - abgerufen am 22.5.2014). 
Abbildung 27: Multiple Korrespondenzanalyse - Positionen in der Freiwilligenarbeit im Feld des Sozialen und der Gesundheit

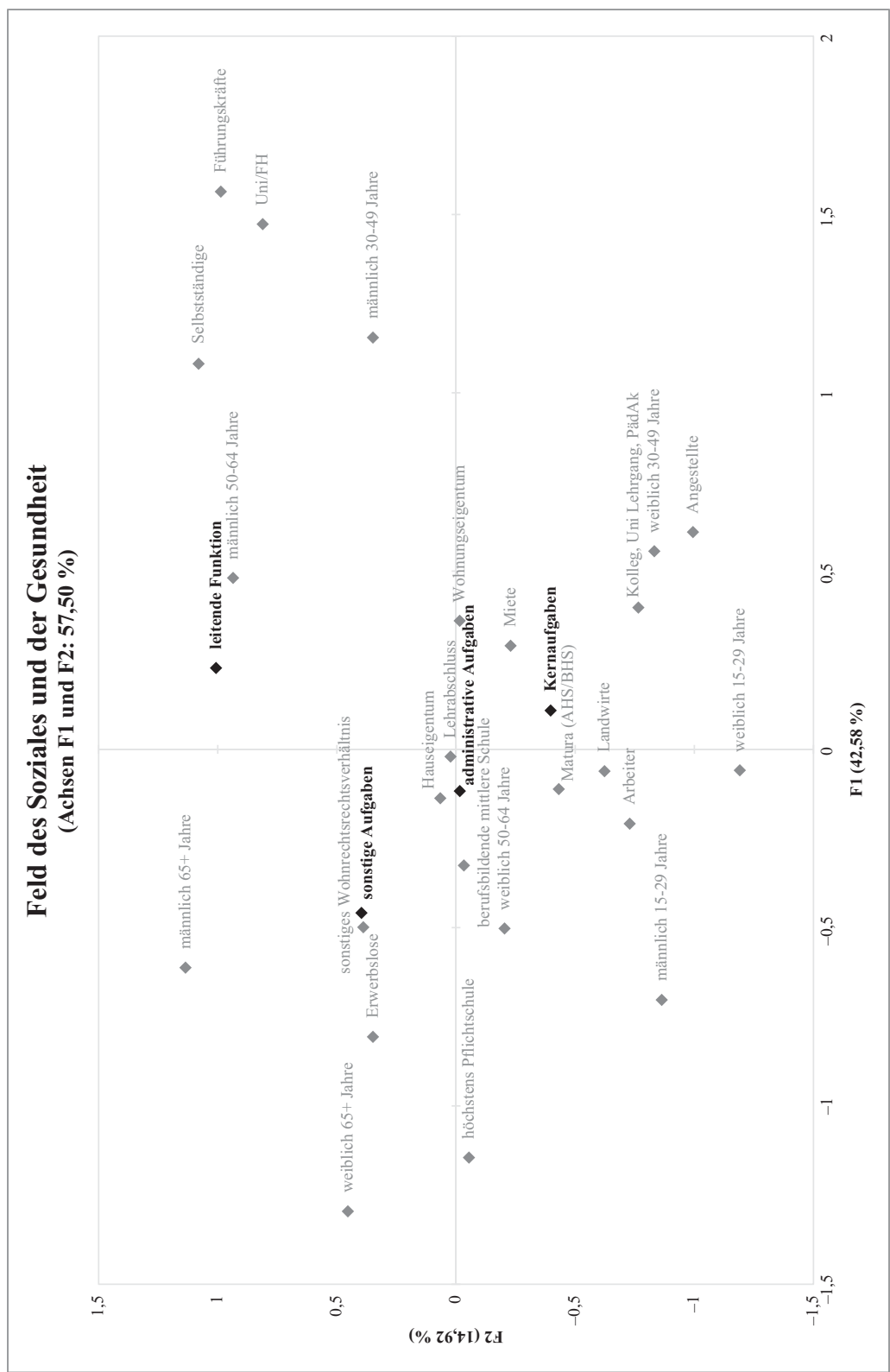


Abbildung 28: Multiple Korrespondenzanalyse - Positionen in der Freiwilligenarbeit im Feld der Kirche und Religion

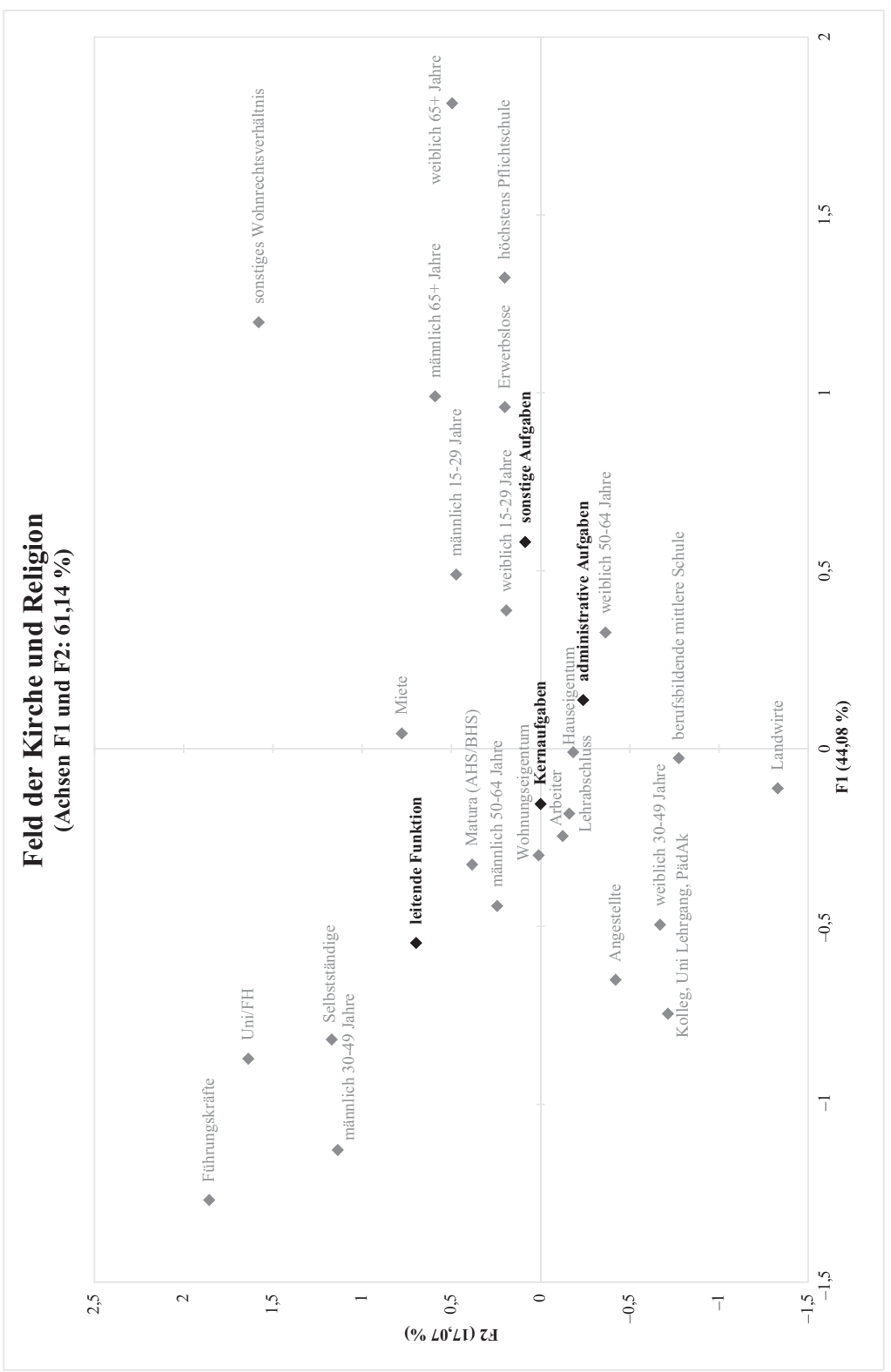


Die grafische Darstellung (Abbildung 27) der Ergebnisse der multiplen Korrespondenzanalyse (MCA) zu den Aufgaben und Positionen im Feld des Sozialen und der Gesundheit zeigt deutlich, dass Positionen mit Leitungsfunktion überdurchschnittlich oft mit Männern zwischen 50 und 64 Jahren besetzt sind. Darüber hinaus geht eine ehrenamtliche Leitungsfunktion überdurchschnittlich oft Hand in Hand mit einer Führungsfunktion im Beruf, beruflicher Selbständigkeit sowie mit einem Universitäts- oder Fachhochschulabschluss. Die Kernaufgaben werden hingegen überdurchschnittlich oft von Freiwilligen mit einem AHS oder BHS Abschluss ausgeführt, sowie von Landwirten und Arbeiterinnen und Arbeitern. Kernaufgaben werden auch überdurchschnittlich oft von Frauen im Alter zwischen 30 und 49 Jahren, die über einen Abschluss eines Kollegs, eines Universitätslehrgangs oder einer Pädagogischen Akademie verfügen und in einem Angestelltenverhältnis berufstätig sind, ausgeführt. Sonstige Aufgaben übernehmen vorwiegend Erwerbslose (z.B. Frauen über 65 Jahre). Damit sind die Erwerbstätigkeit und der Beruf zwar nicht für den Zugang entscheidend, sehr wohl jedoch für die Funktions- und Positionsbesetzung. Darüber hinaus wird deutlich, dass obwohl betreffend des Geschlechts scheinbar keine Zugangsbarrieren zur Freiwilligenarbeit im Feld des Sozialen und der Gesundheit bestehen, die interne Strukturierung hinsichtlich Aufgaben und Funktionen den klassischen stereotypen Rollenmustern der Erwerbsarbeit und auch der Familienarbeit folgt. Sehr gut ausgebildete Männer mittleren bis höheren Alters werden mit Leitungsfunktionen betraut, die Kernaufgaben sowie die administrativen Aufgaben übernehmen überproportional häufig Frauen mit mittlerer Ausstattung an kulturellem Kapital (berufsbildende mittlere Schule oder Matura).

\section{(III) 4.4.6. Positionen im Feld der Kirche und Religion}

Im Jahr 2006 waren hochgerecht knapp 429.000 Freiwillige im Feld der Kirche und Religion freiwillig engagiert. Organisationen, die diesem Feld zugerechnet wurden sind u.a. Pfarrgemeinderäte, Gremien der verschiedenen Religionsgemeinschaften, religiöse Kinder- oder Jugendgruppen bzw. Frauen und Männerbewegungen (vgl. More-Hollerweger/Sprajcer 2009: 40).

Die multivariate Analyse der Determinanten des Zugangs zum Feld der Kirche und Religion (siehe Tabelle 31) zeugt abermals von der hohen Bedeutung des kulturellen Kapitals, d.h. des Bildungsgrads für ehrenamtliches Engagement. Erwartungsgemäß hat verheiratet zu sein, d.h. in der überwiegenden Zahl der Fälle das heilige Sakrament der Ehe empfangen zu haben, in diesem Feld den vergleichsweise größten Einfluss. Zusätzlich spielt das Wohnrechtsverhältnis eine entscheidende Rolle. Wohneigentum und die damit verbundene stärkere Verwurzelung 
im Umfeld fördern den Zugang zu kirchlichem und religiösem Engagement. In diesem Zusammenhang dürfte auch die kulturelle Verwurzelung einen bedeutsamen Einfluss haben. Personen, die nicht in Österreich geboren sind, haben eine fast um die Hälfte geringe Wahrscheinlichkeit in den traditionellen kirchlichen und religiösen Vereinigungen freiwillig tätig zu sein. Bezogen auf den Berufsstatus ist auffällig, dass eine leitende Funktion im Beruf der Freiwilligenarbeit in diesem Feld entgegenwirkt. Sind Führungskräfte allerdings in kirchlichen und religiösen Organisationen aktiv, dann bekleiden sie überdurchschnittlich häufig ein leitendes Ehrenamt.

Das Feld der Religion und der Kirche ist dabei jenes Feld der Freiwilligenarbeit mit dem höchsten Frauenanteil (66,4\%) (siehe Anhang: Tabelle 41). Der Frauenanteil reduziert sich jedoch bei den leitenden Positionen auf rund 56\%. Bourdieu (2005) führt dies in diesem Feld auf die bei bestimmten Bevölkerungsgruppen noch immer vorherrschende Arbeitsteilung zwischen den Geschlechtern zurück, wo für Frauen mangels eigenem Erwerbseinkommen „die Äquivalenz zwischen der Arbeit und ihrem in Geld ausgedrückten Wert nicht eindeutig feststeht" (Bourdieu 1998: 192). Dieser Befund bestätigt sich auch in der multiplen Korrespondenzanalyse: Betrachtet man die MCA in Abbildung 28 wird ersichtlich, dass trotz des hohen Frauenanteils die interne Hierarchisierung den klassischen Rollenmustern folgt. Ehrenamtliche Leitungsfunktionen im Feld der Kirche und der Religion werden analog zum Feld des Sozialen und der Gesundheit überdurchschnittlich oft von Männer im Alter zwischen 30 und 49 Jahren, mit Universitäts-oder Fachhochschulabschlüssen besetzt, die in ihrem Beruf ebenfalls Führungspositionen bekleiden oder aber selbstständig tätig sind. Administrative Aufgaben hingegen erfüllen überdurchschnittlich oft, ebenfalls den traditionellen Rollenmustern folgend, Frauen im Alter zwischen 50 und 64 Jahren mit mittleren bis unteren Bildungsabschlüssen. Freiwillige, die über ein geringeres kulturelles Kapital verfügen (höchstens Pflichtschule) sowie die erwerbslos sind, was oftmals auch Hand in Hand geht, ordnen sich selbst überdurchschnittlich häufig der Residualkategorie der sonstigen Aufgaben zu. Über die Tätigkeiten, die die Kategorie der sonstigen Aufgaben umfasst, lässt sich mangels verfügbarer Daten nur spekulieren. Fehlendes Organisationswissen, dass zur Zuordnung der eigenen Tätigkeiten zu den Antwortkategorien nötig ist, könnte eine Erklärung für diese beobachtete Korrespondenz darstellen. Auch die Gruppe der 15- bis 29-Jährigen (Männer wie Frauen) findet sich überdurchschnittlich mit sonstigen Aufgaben, die anzunehmender Weise mit geringem Status behaftet sind, betraut. In diesem Zusammenhang wäre weiterführend noch interessant gewesen, die Variable zum Familienstand der MCA hinzuzufügen um zu sehen, mit welchen Aufgaben und 
Tätigkeiten die nicht verheirateten Freiwilligen betraut sind. Gesamt betrachtet zeigen die Ergebnisse, dass sich im Feld der Religion und Kirche nicht nur traditionelle Rollen- und Funktionszuweisungen das Geschlecht betreffend, sondern auch das Alter betreffend reproduzieren bzw. auch ihren Ausgang nehmen.

\section{(III) 4.4.7. Positionen im Feld des Sports und der Bewegung}

Mit hochgerechnet rund 475.000 Freiwilligen stellt das Feld des Sports und der Bewegung den zweitgrößten Bereich in der österreichischen Freiwilligenarbeit dar. Die Freiwilligen sind überwiegend in Sportvereinen tätig, die den drei Dachverbände, ASVÖ (Allgemeiner Sportverband Österreich) ${ }^{84}$, ASKÖ (Arbeitsgemeinschaft für Sport und Körperkultur in Österreich ${ }^{85}$ und der Sportunion Österreich ${ }^{86}$ zugeordnet werden können. Die Ergebnisse bestehender Studien legen nahe, dass es gerade im Feld des Sports zu einer Reproduktion von hierarchischen Positionen der Erwerbsarbeit und damit von Prestige und Status kommt (vgl. Braun 2003c; Hartmann-Tews/Combrink 2006; Hovden 2000; Nagel 2003a, b). Der Frauenanteil unter den Freiwilligen im Sport mit rund 31\% wird nur noch im Feld der Katastrophenhilfs- und Rettungsdienste (17\%) unterboten. Im Feld des Sports und der Bewegung werden schließlich nur rund 17\% der ehrenamtlichen Leitungspositionen mit Frauen besetzt (siehe Anhang: Tabelle 42). Wie zu erwarten zeigt die MCA (Abbildung 29), dass auch im Feld des Sports Männer, zwischen 30 und 49 Jahren, mit einer hohen Ausstattung an kulturellem Kapital, die beruflich als Führungskräfte oder als Selbstständige tätig sind, überdurchschnittlich oft mit ehrenamtlichen Leitungsfunktionen betraut sind. Darüber hinaus scheinen auch Personen mit einem Lehrabschluss, die beruflich erfolgreich sind den Sprung ins leitende Ehrenamt, d.h. in Funktionärspositionen im Sport zu schaffen. Administrative Aufgaben korrespondieren wie auch schon in den ersten beiden vorangegangen Feldanalysen mit Frauen mittleren Alters mit mittlerer Ausstattung an kulturellem Kapital. Die Kategorie der sonstigen Aufgaben findet sich wiederum überdurchschnittlich oft bei Männern unter 29 Jahren und über 65 Jahren sowie bei Erwerbslosen und den bildungsfernsten Gruppen.

84 über 1 Million Mitglieder in rund 5.400 Vereinen (http://www.asvoe.at - abgerufen am 28.5. 2014).

85 ca. 1,2 Millionen Mitgliedern in ca. 4.200 Mitgliedsvereine (http://www.askoe.at abgerufen am 28.5. 2014).

86865.000 Mitglieder in 3.803 Vereinen (http://sportunion.at - abgerufen am 28.5. 2014). 
Analysiert man die aktuelle Geschlechterverteilung in Leitungsgremien der drei großen Sportdachverbände in Österreich, hat sich zumindest an den $\mathrm{Zu}-$ gangskriterien zur Führungsspitze seit 2006 kaum etwas verändert.

Tabelle 33: Geschlechterverteilung in den Leitungsgremien der österreichischen Sportdachverbände

\begin{tabular}{|c|c|c|c|c|c|c|c|c|c|}
\hline & \multicolumn{3}{|c|}{ ASKÖ } & \multicolumn{3}{|c|}{ ASVÖ } & \multicolumn{3}{|c|}{ UNION } \\
\hline & $\mathbf{N}$ & $\begin{array}{l}\text { Frauen- } \\
\text { Anteil }\end{array}$ & $\begin{array}{c}\text { Akademiker- } \\
\text { Anteil }\end{array}$ & $\mathbf{N}$ & $\begin{array}{l}\text { Frauen- } \\
\text { Anteil }\end{array}$ & $\begin{array}{c}\text { Akademiker- } \\
\text { Anteil }\end{array}$ & $\mathbf{N}$ & $\begin{array}{l}\text { Frauen- } \\
\text { Anteil }\end{array}$ & $\begin{array}{c}\text { Akademiker- } \\
\text { Anteil }\end{array}$ \\
\hline Präsidium & 21 & $19 \%$ & $29 \%$ & 24 & $4 \%$ & $33 \%$ & 10 & $30 \%$ & $30 \%$ \\
\hline Vorstand & 5 & $20 \%$ & $80 \%$ & 5 & $0 \%$ & $60 \%$ & 10 & $60 \%$ & $40 \%$ \\
\hline $\begin{array}{l}\text { Sportaus- } \\
\text { schuss }\end{array}$ & 22 & $14 \%$ & $36 \%$ & 9 & $0 \%$ & $33 \%$ & k.A. & k.A. & k.A. \\
\hline
\end{tabular}

Quellen: http://www.askoe.at;. http://www.asvoe.at; http://sportunion.at; Stand Februar 2014.

Die Bandbreite des Anteils von Frauen in den Präsidien reicht von rund 19\% (ASKÖ) bis hin zu rund 4\% (ASVÖ). Im Vorstand der ASKÖ findet sich eine Frau, im Vorstand des ASVÖ hingegen keine einzige Frau. Die UNION bildet bei der Geschlechterverteilung im Vorstand die Ausnahme, mit einem Frauenanteil von $60 \%$. Der Akademikeranteil liegt bei den Vorständen der Dachverbände zwischen $40 \%$ und $80 \%$. In den anderen Gremien liegt der Akademikeranteil durchschnittlich bei einem Drittel. Geschlecht und Bildungsgrad bilden damit neben dem Berufsstatus, weiterhin auch die zentralen Kriterien der internen hierarchischen Strukturierung im Feld des Sports.

Im weiterhin männerdominierten und vorwiegend auf die eigenen Mitglieder ausgerichteten Feld des Sports kommt es zur Reproduktion und möglicherweise auch Verstärkung sozial ungleicher Macht- und Handlungsspielräume. Die Gründe dafür mögen, analog zum Wirtschafts- wie Wissenschaftssystem, folgende sein: Unter dem Deckmantel der Chancengleichheit und der Leistungsgerechtigkeit, die durch den Wettkampf (im Sport wie am freien Markt) abgesichert und objektiviert wird, haben ungleichheitsgenerierende Prozesse (Matthäus-Effekt, statistischen Diskriminierung, etc.) freie Hand und sind nur durch eine bewusst gestaltete Regeländerung (wie z.B. durch Quotenregelungen) veränderbar. Die Illusion der Chancengleichheit ist prototypisch für das Feld des Sports und gilt scheinbar auch für die vielfach ehrenamtliche Organisation desselben. Damit wäre erklärbar warum derartige soziale Schieflagen betreffend den Zugang zu Leitungspositionen persistent in die Regeln des Feldes eingeschrieben und sich bis heute vielfach als legitimiert unsichtbar gemacht haben. 
Abbildung 29: Multiple Korrespondenzanalyse: Positionen in der Freiwilligenarbeit im Feld des Sports und der Bewegung

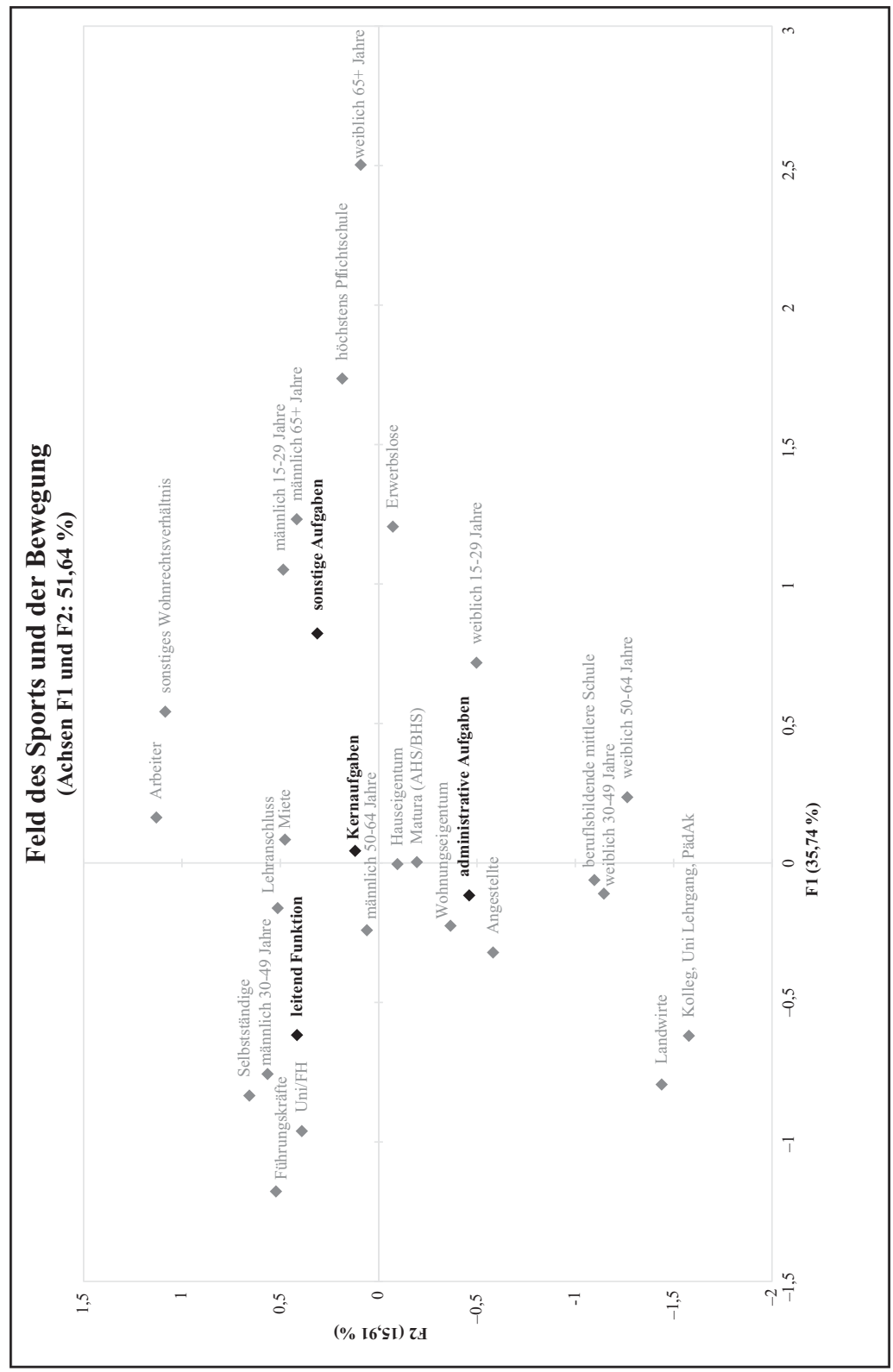


Abbildung 30: Multiple Korrespondenzanalyse: Positionen in der Freiwilligenarbeit im Feld der Katastrophenhilfs- und Rettungsdienste

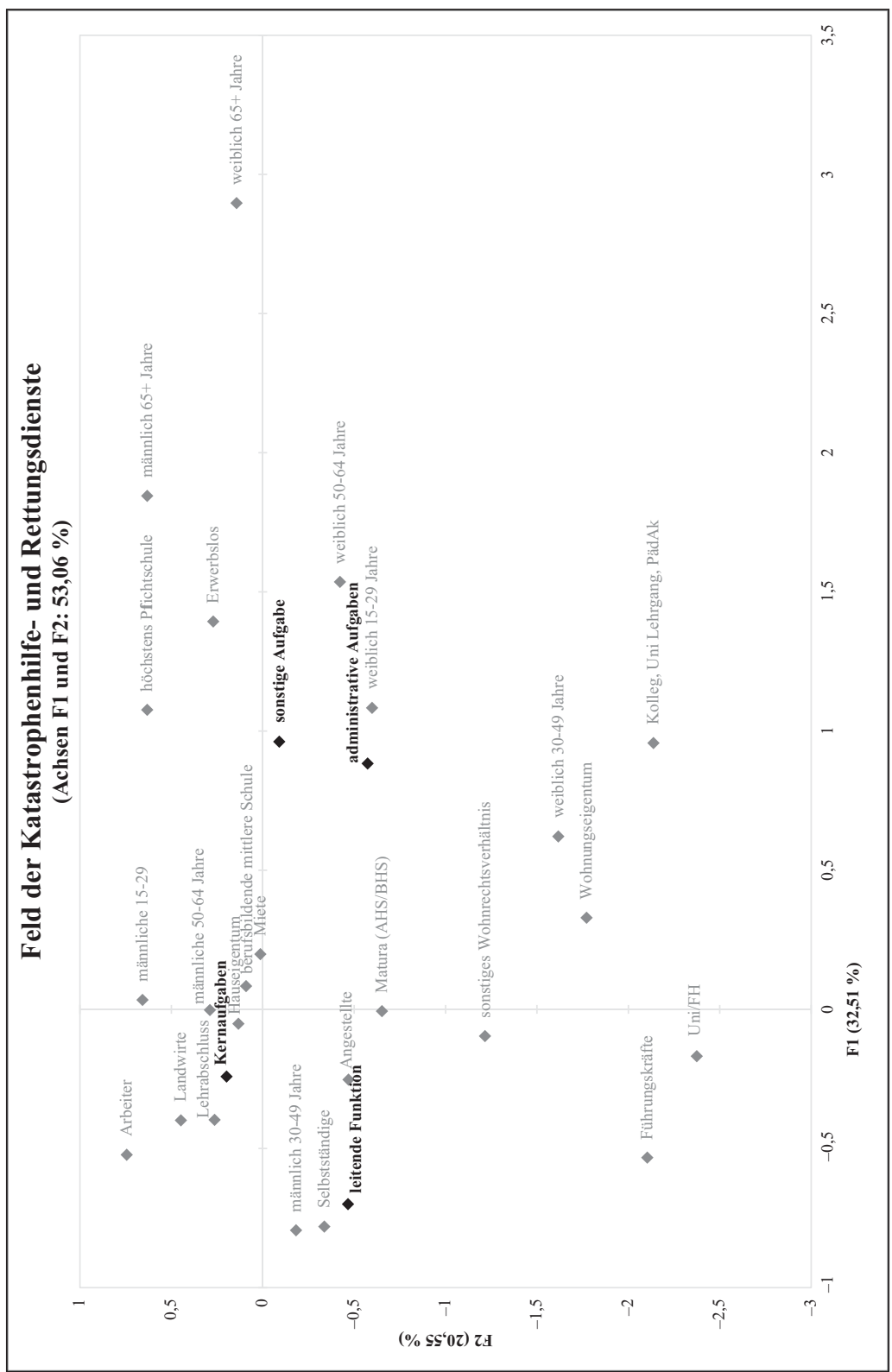




\section{(III) 4.4.8. Positionen im Feld der Katastrophenhilfs- und Rettungsdienste}

Im Feld der Katastrophenhilfs- und Rettungsdienste waren im Jahr 2006 rund 413.000 Freiwillige tätig. Damit ist dies der drittgrößten Bereich der Freiwilligenarbeit in Österreich (More-Hollerweger/Sprajcer 2009: 36f). Das Feld umfasste zum Zeitpunkt der Erhebung (Stand 2007) 4.527 Freiwillige Feuerwehren ${ }^{87}$, die Rettungsdienste des Roten Kreuz, des Arbeiter-Samariter Bundes, des Grünen Kreuzes, des Malteser Hospitaldienstes ${ }^{88}$ sowie der Johanniter-Unfall-Hilfe. Darüber hinaus zählt dazu auch die Bergrettung ${ }^{89}$, die Rettungshundebrigade ${ }^{90}$, die Wiener Wasserrettung sowie die österreichische Höhlenrettung. Der Frauenanteil unter den Freiwilligen in diesem Feld liegt bei rund 17 \%, unter den leitenden Positionen sinkt er auf rund 8\%. Die vergleichsweise wenigen Frauen im Feld sind erwartungsgemäß überdurchschnittlich oft mit administrativen Aufgaben betraut (Abbildung 30). Sowohl leitende Funktionen als auch Kernaufgaben werden primär von Männern ausgeführt. Leitende Funktionen korrespondieren mit Männern im Alter zwischen 30 und 49 Jahren, die selbstständig oder in einem Angestelltenverhältnis tätig sind, und über mittlere bis hohe Bildungsabschlüsse verfügen. Die Kernaufgaben werden wiederum überdurchschnittlich oft von Männern im Alter zwischen 15 und 29 sowie zwischen 50 und 64 Jahren mit mittlerem Bildungsabschluss (Lehrabschluss, berufsbildende mittlere Schule) ausgeführt, die beruflich als Arbeiter oder Landwirte tätig sind. Damit spiegeln sich in der Freiwilligenarbeit im Feld der Katastrophenhilfs- und Rettungsdienste nicht nur Geschlechterstereotypen hinsichtlich der Aufgabenverteilung wieder, sondern generell die Strukturierung und Hierarchisierung des Erwerbsarbeitsfeldes nach Bildungstiteln.

Auch eine Studie zur Sozialstruktur im Österreichischen Roten Kreuz (vgl. Czech 2010) aus dem Jahr 2010, die sowohl Ehrenamtliche als auch Hauptamtliche beinhaltet, kommt zu dem Ergebnis, dass der Frauenanteil mit steigender Dienstrangklasse signifikant abnimmt. Liegt der Frauenanteil bei den sogenannten Mannschaftsgraden noch bei 33,4\% sinkt dieser bei den Offiziersrängen auf $24 \%$. Schließlich findet sich unter den sieben höchsten Funktionsträgern im Roten Kreuz Niederösterreich nur eine Frau (14, 3\%) (ebd: 65). Auch der Einfluss des kulturellen Kapitals auf die interne Hierarchisierung findet sich bestätigt. So sinkt der Anteil an Personen mit höchstens Maturaabschluss von rund $82 \%$ bei den Mannschaftsgraden auf 33\% bei den Offiziersrängen und weiter auf 14,3\%

87249.507 Freiwillige.

88300 Freiwillige.

8911.211 Freiwillige.

90724 Freiwillige. 
bei den höchsten Funktionsträgern. Der Anteil der Akademikerinnen und Akademiker bei den Offiziersrängen liegt bei 43\% und bei den höchsten Funktionsträgern bei rund $71 \%$. Obwohl in dieser Studie bei der Analyse nicht zwischen Ehrenamtlichen und Hauptamtlichen unterschieden wurde, wird deutlich, das die Regeln des Zugangs wie der internen Hierarchisierung im Feld der Rettungsdienste auch für die Freiwilligen Geltung besitzen dürften.

Der geringe Frauenanteil und die Regeln der Positionsbesetzung im Feld der Katastrophenhilfs- und Rettungsdienste kann zu einem Gutteil damit erklärt werden, dass sich die meisten der Organisationen in diesem Feld „quasimilitärischer" hierarchischer Strukturen bedienen. Dies erklärt mitunter auch den vergleichsweise geringen Anteil an Personen der oberen Bildungsschichten, die militärischen Organisationstrukturen, ungeachtet deren Funktionalität, eher kritisch gegenüber stehen.

\section{(III) 4.4.9. Zusammenfassung der Ergebnisse zu den ausgewählten Feldern}

Die Ergebnisse zu den Feldern der Freiwilligen machen deutlich, dass es selbst in jenen Feldern wie dem Sozialen und der Religion, die hinsichtlich unterschiedlicher sozialer Merkmale beim Zugang eine vergleichsweise geringe Selektivität aufweisen, im Rahmen der internen Hierarchisierung zu einer Reproduktion bestehender Muster sozial ungleicher Strukturierung nach Geschlecht, Alter, Berufsstatus und Bildungsgrad kommt. Hinsichtlich der Bedeutung der Kapitalien lassen sich die Ergebnisse folgendermaßen zusammenfassen:

Institutionalisiertes kulturelles Kapital. Je höher der Bildungsabschluss, desto höher ist der Beteiligungsgrad an formeller Freiwilligenarbeit. Dieser Einfluss bleibt selbst unter Bedacht der andern Kapitalformen sowie der Kontrolle für Alter, Geschlecht und Bevölkerungsdichte signifikant. Institutionalisiertes kulturelles Kapital in Form von Bildungsabschlüssen variiert jedoch in seiner Bedeutung, je nach dem, in welchem Feld das Freiwillige Engagement erfolgt. Für den Zugang hat es in den analysierten Feldern, bis auf jenes der Katastrophenhilfs- und Rettungsdienste, einen positiven Einfluss. Für die interne Hierarchisierung, d.h. konkret für den Zugang zu Positionen mit leitender Funktion, ist ein hoher Bildungsabschluss in allen vier analysierten Feldern entscheidend. Somit bildet institutionalisiertes kulturelles Kapital in allen vier Feldern, wenn auch auf unterschiedlichem Niveau, eine zentrale Voraussetzung für Freiwilliges Engagement. Allen vier Feldern gemein ist auch die geringe Chance des Zugangs zu leitenden Funktionen von Personen der untersten Bildungsschichten, d.h. jener mit der geringsten Ausstattung an kulturellem Kapital. 
Ökonomisches Kapital. Oftmals wird Freiwilligenarbeit auch als Spende von Zeit bezeichnet. Die Spende von Zeit setzt jedoch voraus, dass man über die eigene Zeit auch tatsächlich frei verfügen kann, um sie infolge auch spenden zu können. Was Bourdieu für den Erwerb von kulturellem Kapital beschrieben hat, scheint den empirischen Ergebnissen zur Folge auch für die Freiwilligenarbeit zuzutreffen: Es bedarf, „,von ökonomischen Zwängen befreite Zeit“ (Bourdieu 1983, S. 188). Gesamt gesehen hat ökonomisches Kapital in Form von Wohneigentum einen signifikant positiven Einfluss auf den Zugang zur Freiwilligenarbeit, nicht jedoch für die weitere interne Hierarchisierung. Nach Feldern differenziert zeigt sich, dass die Bedeutung von ökonomischer Absicherung in Form von Wohneigentum nicht konsistent die gleiche Bedeutung im Kontext der anderen Kapitalien hat. Im Feld des Sports und der Bewegung, der Kirche und Religion sowie der Katastrophenhilfs- und Rettungsdienste beeinflusst Wohneigentum positiv den Zugang zu Freiwilligenarbeit, im Feld des Sozialen und der Gesundheit hat diese Form des ökonomischen Kapitals hingegen keinen signifikanten Einfluss.

Symbolisches Kapital. Die Bedeutung des symbolischen Kapitals im Kontext der Freiwilligenarbeit wurde seit der Begriffswandlung weg vom „Ehrenamt“ und hin zu „Freiwilligenarbeit" und „Freiwilligem Engagement" zunehmend aus dem Blick verloren. Damit haben sich scheinbar auch die Perspektive und die Aufmerksamkeit von der „Statuskomponente" des Ehrenamts (vgl. Weber 1921/1980) auf die „Sozialkapitaldimension“ des Freiwilligen Engagements verschoben. Wie eng jedoch weiterhin die Freiwilligenarbeit von statusbezogenen sozio-ökomischen Dimensionen beeinflusst ist, haben die empirischen Ergebnisse zur Bedeutung der beruflichen Position beim Zugang zu leitenden Funktionen in der Freiwilligenarbeit gezeigt.

\section{(III) 5. Zusammenfassung der zentralen empirischen Ergebnisse}

\section{(III) 5.1. Ergebnisse zur sozialen Schließung}

Der Zugang zur Freiwilligenarbeit ist in Österreich sowohl hinsichtlich zugeschriebener als auch erworbener sozialer Merkmale selektiv und damit durch soziale Schließung gekennzeichnet. Einerseits sind angeborene (d.h. zugeschriebene) Merkmale wie Geschlecht, Alter und ethnische Herkunft für den Zugang zur Freiwilligenarbeit entscheidend. So sind Frauen, ältere Menschen sowie Migrantinnen und Migranten aufgrund von Zugangsbarrieren und sozialer Schließungsmechanismen seltener freiwillig engagiert. Andererseits entscheiden auch erworbene soziale Merkmale wie der Bildungsgrad, die Vermögensausstattung und der Familienstand über den Zugang zur Freiwilligenarbeit. Ein 
geringer Bildungsgrad, d.h. eine geringere Ausstattung an kulturellem Kapital erschwert den Eintritt in die Freiwilligenarbeit. Die Erwerbstätigkeit alleine hat keinen Einfluss auf das Engagement, in Verbindung mit einer Leitungsfunktion wirkt sie allerdings förderlich. Die Vermögensausstattung in Form von Wohneigentum stellt darüber hinaus einen zentralen positiven Einflussfaktor für den Zugang zur Freiwilligenarbeit dar. Unverheiratet zu sein sowie in Regionen mit einem hohen Urbanisierungsgrad zu leben verringert hingegen die Wahrscheinlichkeit für ein Ehrenamt. Weiterführend hat die Gegenüberstellung der Determinanten des Engagements von Männern und Frauen gezeigt, dass für Frauen der Bildungsgrad und der Familienstand und für Männer eine leitende Funktion im Beruf und die Vermögensausstattung (Wohneigentum) eine vergleichsweise höhere Bedeutung für den Zugang zur Freiwilligenarbeit haben. Kinder unter 3 Jahren wirken sich primär negativ auf das Freiwillige Engagement von Frauen aus, Kinder zwischen 3 und 15 Jahren alt fördern wiederum vorwiegend das Engagement von Frauen. Frauen und Migrantinnen und Migranten sind seltener in mehreren Organisationen freiwillig tätig als Männer sowie in Österreich geborene Personen. Die für Mehrfachengagement förderlichen Ressourcen bzw. Kapitalien umfassen einen höheren Bildungsgrad, eine leitende Funktion im Beruf und einen Ehepartner. Die Vermögensausstattung in Form von Wohneigentum spielt für Mehrfachengagement keine bedeutsame Rolle. Der Vergleich der Determinanten des Zugangs zur Freiwilligenarbeit mit jenen des Zugangs zur Erwerbsarbeit hat gezeigt, dass die beiden Felder ihrer Zugangsregeln betreffend vorwiegend kongruente und nicht komplementäre bzw. alternative Tätigkeitsfelder darstellen. Die Analyse der subjektiven Barrieren der Freiwilligenarbeit und deren Determinanten hat gezeigt, dass die in den sozialen, ökonomischen, kulturellen und symbolischen Dimensionen benachteiligten Bevölkerungsgruppen ihre diesbezügliche Schlechterstellung auch subjektiv als Barrieren für Zugang zur Freiwilligenarbeit wahrnehmen. Gesamt betrachtet deuten die Ergebnisse darauf hin, dass der sozial selektive Zugang zur Freiwilligenarbeit zu einer Verfestigung und teilweisen Verstärkung des allgemeinen Zustandes sozialer Ungleichheit beiträgt.

\section{(III) 5.2. Ergebnisse zur internen Hierarchisierung}

Die Positionsbesetzung in der Freiwilligenarbeit folgt in Österreich klassischen Mustern der Hierarchisierung. Was Bourdieu (1998) über die Hierarchisierung und Aufgabenverteilung innerhalb des kirchlichen Ehrenamts geschrieben hat, trifft damit in Grundzügen auf alle Felder der Freiwilligenarbeit zu. Frauen haben eine, um fast die Hälfte geringere Chance, in ehrenamtliche Leitungspositionen zu kommen. Der Bildungsgrad, eine Erwerbstätigkeit und dabei in 
besonderem Maße eine leitenden Funktion, sind von zentraler Bedeutung für den Zugang zu leitenden Positionen im Ehrenamt. So haben beispielsweise in der Freiwilligenarbeit Angehörige der bildungsfernsten Schichten sowie Arbeitslose kaum die Möglichkeit Leitungs- bzw. Führungserfahrungen zu sammeln. Der Vergleich der Determinanten der Hierarchisierung in der Freiwilligenarbeit mit jenen in der Erwerbsarbeit macht deutlich, dass die beiden Tätigkeitsfelder nicht nur über ähnliche Zugangsregeln, sondern ebenso über ähnliche Regeln der internen Strukturierung verfügen. Mehrfachengagement und Leitungsfunktion gehen vielfach Hand in Hand. Die diesbezüglichen Kausalitäten sind ohne entsprechende Daten (z.B. Längsschnitterhebungen) jedoch nicht zu entflechten. Sowohl Mehrfachengagement als auch leitende Positionen verlangen nach einer vergleichsweise hohen Ausstattung an kulturellem und symbolischem Kapital. Es ist daher anzunehmen, dass hier, sich wechselseitig verstärkende, Effekte der Kapitalakkumulation (Matthäus-Effekt) wirksam sind. Der Vergleich der Determinanten der internen Hierarchisierung bei Männern und Frauen hat gezeigt, dass für Frauen ein höherer Bildungsgrad und für Männer eine leitende Funktion im Beruf den Zugang zu leitenden Positionen in der Freiwilligenarbeit begünstigen. Die Reproduktion von Prestige und Status der Erwerbsarbeit in der Freiwilligenarbeit ist vorwiegend bei den Männern zu beobachten. Freiwilligenarbeit ist als Quelle für symbolisches und soziales Kapital oftmals über den gesamten Lebenslauf wirksam und führt, im Gegensatz zu beruflichen Karrieren, zu einer meist verschleierten Akkumulation und Transformation statusrelevanter Kapitalien.

\section{(III) 5.3. Ergebnisse zur sozialen Schließung und Hierarchisierung in den Feldern}

Die soziale Selektivität ist in Österreich in den Feldern der Freiwilligenarbeit unterschiedlich stark ausgeprägt. Die Hierarchisierung folgt hingegen selbst in den weniger selektiven Feldern den klassischen Hierarchisierungsmustern der Erwerbsarbeit. Die Felder des Sozialen und der Religion sind beim Zugang hinsichtlich der sozialen Merkmale vergleichsweise offen, im Rahmen der internen Hierarchisierung werden das Geschlecht, das Alter, der Berufsstatus und der Bildungsgrad jedoch bedeutsam und Ausgangspunkte von Ungleichbehandlungen. Das Engagement im Sport und in der Katastrophenhilfe folgt bereits beim Zugang und in hohem Ausmaß bei der Positionsbesetzung der tradiert persistenten, stereotypen Rollenaufteilung zwischen den Geschlechtern. Die Männer mittleren bis höheren Alters übernehmen Führungsfunktionen und die Kernaufgabengebiete, die ohnehin schon wenigen Frauen in diesen Feldern 
finden sich in unterstützenden und administrierenden Aufgabengebieten wieder. Allen vier Feldern gemein ist die geringe Chance von Personen der untersten Bildungsschichten beim Zugang zu leitenden Funktionen. Für den Zugang zur Freiwilligenarbeit ist die Vermögensausstattung in Form von Wohneigentum in allen analysierten Feldern bis auf das Feld des Sozialen und der Gesundheit von Bedeutung. Die interne Hierarchisierung wird jedoch in keinem der Felder von dieser Form der Vermögensausstattung beeinflusst. Die Ergebnisse zur Bedeutung der beruflichen Position beim Zugang zu leitenden Funktionen in den untersuchten Feldern haben gezeigt, wie stark weiterhin die Freiwilligenarbeit von statusbezogenen Dimensionen beeinflusst wird. Die Felder tragen somit, in unterschiedlicher Form und in unterschiedlichem Ausmaß, zur Reproduktion sozialer Ungleichheit bei.

\section{(III) 6. Limitationen}

Die Limitationen betreffen vorwiegend die aus ungleichheitstheoretischer Perspektive geforderte kausalanalytische Betrachtung des Zusammenhangs von ungleichem Zugang und den damit verbundenen ungleichen Auswirkungen. Art und Umfang der verwendeten Sekundärdaten haben diesen Analyseschritt nicht ermöglicht. Die Auswirkungen des sozial ungleichen Zugangs und der Hierarchisierung konnten somit nur auf Basis des Stands der Forschung beurteilt und nicht in die eigene empirische Analyse integriert werden. Die Frage, ob es sich bei Freiwilligem Engagement um eine (neue) Dimension sozialer Ungleichheit handelt muss damit empirisch unbeantwortet bleiben. Eine weitere Einschränkung betrifft die Übertragbarkeit der Daten auf Organisationebene. Die empirischen Sekundärdaten wurden auf Bereichs- bzw. Feldebene, nicht aber auf Organisationsebene erfasst. Die Ergebnisse sind daher primär als Anregung für die einzelnen Organisationen gedacht, ihre impliziten wie expliziten Zugangsregeln einer kritischen Reflexion zu unterziehen und weniger, bestimmten Organisationen grundlegend ihr integratives Potential abzusprechen. 



\section{(IV) Schlussfolgerungen}

Die Ergebnisse der vorliegenden Arbeit, d.h. der theoretischen Erörterung in Kapitel (1) sowie der empirischen Analysen in Kapitel (III) verweisen auf die verborgenen (negativen) Konsequenzen und die nichterfüllbaren Erwartungen, die an die Freiwilligenarbeit derzeit gerichtet werden. Die daraus abgeleiteten Schlussfolgerungen gliedern sich 1) in die Relevanz für die weitere Forschung und 2) in die Relevanz für Akteure und Handlungsfelder auf individueller, organisationaler, politischer und gesamtgesellschaftlicher Ebene. Den Abschluss bildet ein allgemeines Fazit.

\section{(IV) 1. Relevanz der Ergebnisse für die weitere Forschung}

Die empirischen Ergebnisse zur Reproduktion sozialer Ungleichheit in der Freiwilligenarbeit leisten einen wichtigen Beitrag zur differenzierten Erforschung der Determinanten der Freiwilligenarbeit und eröffneten darüber hinaus einen kritischen Blick auf den Zusammenhang zwischen den Zugangsvoraussetzung und den Auswirkungen der Freiwilligenarbeit. Insbesondere die Ergebnisse zum Einfluss der sozialen Merkmale auf die interne Hierarchisierung tragen zur weiterführenden Erforschung der verschleierten Tradierung gesellschaftlicher Machtverhältnisse bei. Konkret, hat die ungleichheitstheoretische Perspektive erstmals dazu angeregt, die Forschungsarbeiten zu den Auswirkungen der Freiwilligenarbeit mit ihren Voraussetzungen in Beziehung zu setzen und den Blick auf mögliche ungleichheitsgenerierende Prozesse zu schärfen. Der Nachweis der sowohl feldübergreifenden als auch feldspezifischen Bedeutung der Determinanten bietet ein differenziertes Bild der Selektionseffekte, die in der Freiwilligenarbeit wirksam sind. Die theoretischen Perspektiven und empirischen Ergebnisse zum Zusammenhang von Freiwilligenarbeit und sozialer Ungleichheit öffnen dabei den Blick für den Bedarf an künftigen Forschungsarbeiten, die die Engagementforschung und Ungleichheitsforschung methodisch wie theoretisch stärker miteinander verschränken.

\section{Forschung zu den Effekten der Freiwilligenarbeit}

Um die bestehende Lücke zwischen den Determinanten und den Effekten der Freiwilligenarbeit zu schließen, bedarf es Studien zu den Auswirkungen, die nicht nur für soziale Merkmale kontrollieren, sondern diesbezüglich konkrete Interaktionseffekte in den Blick nehmen, d.h. in welcher Form positive 
(wie negative) Effekte von der Zugehörigkeit zu bestimmten sozialen Merkmalsgruppen abhängen. Für diese Art von Fragestellungen bieten sich neben dem Aufbau eines Freiwilligenpanels auch quasi-experimentelle Forschungsdesigns an. Differenziertere Erkenntnisse zu den Auswirkungen der Freiwilligenarbeit könnten auch deren Beitrag bei der Verstärkung oder aber Verringerung gesamtgesellschaftlicher Ungleichheit besser abschätzen lassen. Abgesehen von Effekten auf den Ebenen der Gesundheit und der sozialen Integration gilt es auch, die künftige Bedeutung von zertifizierten Freiwilligentätigkeiten im Lebenslauf für den Zugang zum Arbeitsmarkt sowie zu Führungspositionen im Beruf in den Blick zu nehmen.

\section{Forschung zum Einfluss gesellschaftlicher Entwicklungen auf den Zugang sowie auf die Effekte der Freiwilligenarbeit}

Die individuellen Auswirkungen der Freiwilligenarbeit unterliegen auch Einflussfaktoren, die auf gesamtgesellschaftlicher Ebene zu verorten sind. Es bedarf unter anderem Forschungsarbeiten dazu, in welcher Form die Zunahme der sozialen Ungleichheit, z.B. in den Dimensionen Einkommen und Vermögen, die Prozesse sozialer Schließung und Hierarchisierung in der Freiwilligenarbeit beeinflussen. Als Beispiel wäre die Frage zu nennen, wie sich das weitere Auseinanderklaffen der Einkommensschere und die derzeitige Preisentwicklung am Wohnungsmarkt auf die künftige Beteiligung an der Freiwilligenarbeit auswirken.

\section{Forschung zu den wechselseitigen Profiten zwischen Freiwilligen und Organisationen}

Ausgehend von den individuellen Erträgen der Freiwilligenarbeit auf symbolischer, kultureller und sozialer Ebene stellt sich auch die Frage, in welcher Form die jeweiligen Freiwilligenorganisationen vom symbolischen Kapital statushöherer Freiwilliger profitieren und welche Karriereverlaufsmuster diesbezüglich zu beobachten sind. Dabei sind nicht nur die wechselseitigen Profite zwischen Ehrenamtlichen und Freiwilligenorganisationen in den Blick zu nehmen, sondern ist auch an die wechselseitige Beeinflussung der beruflichen und der ehrenamtlichen Karrieren im Lebenslauf zu denken.

\section{Forschung zu den konkreten Selektionsmechanismen in den Freiwilligen-organisationen}

Diesbezügliche Fragen betreffen konkret die Auswirkungen der Professionalisierung des Freiwilligenmanagements auf die Diversität in den Freiwilligenorganisationen. Führt der vermehrte Einsatz von Personalmanagementinstrumenten zu einer Verstärkung oder Verringerung sozialer Selektion von Freiwilligen? Konkret, sind mit den Tendenzen der Managerialisierung im Nonprofitsektor 
vorwiegend Effekte sozialer Exklusion oder sozialer Inklusion verbunden. Vor allem qualitativ orientierte Forschungsarbeiten zu den Fremd- wie auch Selbstselektionsmechanismen würden zu einem tieferen Verständnis der Ursachen des sozial ungleichen Zugangs zur Freiwilligenarbeit beitragen.

\section{Forschung zum Social Impact von Drittsektoraktivitäten}

Zunehmend sehen sich auch Freiwilligenorganisationen mit der Aufforderung konfrontiert ihre sozialen Wirkungen (Social Impact) umfassend nachzuweisen. Es bedarf daher weiterer Forschungsarbeiten dazu, wie die Erkenntnisse zur Reproduktion sozialer Ungleichheit in der Freiwilligenarbeit für die Wirkungsmessung in NPOs eingesetzt werden können. Die Ergebnisse wären auch gesamt betrachtet ein wichtiger Bestandteil für künftige Wirkungsmessungen des gesamten Dritten Sektor (Third Sector Impact).

\section{Bedarf an amtlicher Statistik}

Um die genannten Forschungslücken schließen zu können ist die Wissenschaft neben forschungsförderungs- und eigenfinanzierten Studien auch auf die Kooperation mit der amtlichen Statistik angewiesen. Zum Beispiel ist eine differenziertere Erfassung der Freiwilligenarbeit idealerweise nach Organisation, Tätigkeiten, Funktionen, etc. und die Integration von Fragen zur sozialen Herkunft (Bildungsgrad, Freiwilliges Engagement, Beruf, Einkommen, etc.) sowie Fragen zu den Auswirkungen der Freiwilligenarbeit für die künftige Forschung unabdingbar. Wünschenswert wäre darüber hinaus, dass auch in Österreich ein Freiwilligenpanel bzw. Survey ähnlich der Schweiz, Deutschland bzw. den Niederlanden etabliert wird. Nicht zuletzt um die von der Politik geforderten Entwicklungstrends wissenschaftlich abzusichern und die Zugangsvoraussetzungen mit den Auswirkungen gemeinsam analysieren zu können.

\section{(IV) 2. Relevanz der Ergebnisse auf individueller, organisationaler, politischer und gesellschaftlicher Ebene}

Die Ergebnisse der vorliegenden Arbeit sollen jedoch nicht nur neue Forschung anregen, sondern sind für Akteure und Handlungsfelder auf individueller, organisationaler, politischer und gesamtgesellschaftlicher Ebene von unterschiedlicher Bedeutung.

\section{Ebene der Individuen}

Vor dem Hintergrund der beobachteten Zugangsbarrieren gilt es vor allem jene Personen, die den weniger privilegierten Schichten der Gesellschaft zuzurechnen 
sind und die sich nicht freiwillig engagieren, vom Generalverdacht des „NichtWollens" freizusprechen und stattdessen Bedingungen und Strukturen in den Blick zu nehmen die ein „Wollen“ und „Können“ ermöglichen. Die Ergebnisse können aber auch den Individuen selbst als Ausgangspunkt und Reflexionsfolie ihres eigenen freiwilligen Engagements bzw. ihres Nicht-Engagements dienen: Warum bin ich/bin ich nicht ehrenamtlich tätig? Über welche Ressourcen- bzw. Kapitalausstattung verfüge ich? Welche Funktion(en) erfülle ich und welche Position(en) bekleide ich? Wie bin ich in die aktuelle(n) Position(en) gekommen? Welche Vor- und Nachteile sind damit für mich in anderen Lebensbereichen verbunden?

\section{Ebene der Organisationen}

Die Relevanz der Ergebnisse für die Freiwilligenorganisationen betrifft unterschiedliche Bereiche des Managements sowie die Ausgestaltung der GovernanceStrukturen. Auf Ebene des strategischen Managements stellt sich die Frage nach der Passung von Unternehmensmission und den Rekrutierungsstrategien sowie der Diversitätsstruktur der Freiwilligen. Auf der Ebene des operativen (Freiwilligen-)Managements sind die Methoden der Personalgewinnung sowie die konkreten Kriterien der Personalauswahl von Freiwilligen und den damit verbunden (sozialen) Selektionseffekten kritisch zu prüfen. Mit den Ergebnissen soll jedoch auch bei kleineren Vereinen ein kritischer Blick auf die Reproduktion von traditionellen Geschlechterrollen bei der Vergabe von Funktionen und Aufgaben, sowie dessen etwaige Nebenwirkungen, angeregt werden. Organisationen könnten sich betreffend der Diversität ihrer Freiwilligen folgende Fragen stellen: Sind die für die Organisation bedeutsamen Interessensgruppen in der Sozialstruktur des Freiwilligenstaffs abgebildet? Ist die Struktur der Stakeholder in den (ehrenamtlichen) Steuerungsorganen (Governance-Strukturen) entsprechend abgebildet? Welche Bevölkerungsgruppen werden implizit oder explizit vom Freiwilligen Engagement in der Organisation ausgeschlossen? Welche Auswirkungen hat die heutige Selektion von Freiwilligen für die Deckung des zukünftigen Bedarfs an Freiwilligen? Nach welchen Kriterien werden ehrenamtliche Führungspositionen besetzt bzw. zur Wahl ausgeschrieben? Und letztlich, welche (integrativen) Potentiale der Organisation werden der Politik sowie der Gesellschaft (durch Leitbildern und Unternehmensvisionen) suggeriert.

\section{Ebene der Politik}

Auf (gesellschafts-)politischer Ebene stellt die Arbeit ebenfalls einen Ausgangspunkt zur kritischen Reflexion der gesellschaftlichen Integrationsfunktion der Freiwilligenarbeit dar. Darüber hinaus relativieren die Ergebnisse die große 
Bedeutung, die der Freiwilligenarbeit als Handlungsfeld zum Erwerb für berufsrelevante wie demokratiepolitische Kompetenzen zugeschrieben wird. Idealweise werden im künftigen Diskurs über die Zivilgesellschaft die Erwartungen an die Freiwilligenarbeit sowie die damit verbundenen Kausalnahmen hinsichtlich der positiven Effekte neu überdacht und die Potentialeinschätzungen in Zukunft vermehrt an empirischen Evidenzen ausgerichtet. Ausgehend von den empirischen Ergebnissen zu den Determinanten der Freiwilligenarbeit umfassen Empfehlungen an die Politik, ceteris paribus, u.a. folgende Punkte: Arbeitsmarkt- und steuerpolitische Maßnahmen, die zu einer gerechteren Verteilung der Einkommen beitragen fördern auch Freiwilliges Engagement. Investitionen in und Reformen des Bildungssystems, die zu einer Erhöhung des Bildungsgrads der Bevölkerung beitragen, wirken auch positiv auf die Bereitschaft sich freiwillig zu engagieren. Auch die Förderung von finanzierbarem Wohneigentum kann zu einer stärkeren Verwurzelung im jeweiligen Umfeld beitragen und fördert das Freiwillige Engagement und die Bürgerbeteiligung. Sozialpolitik, Steuerpolitik, Familienpolitik, Arbeitsmarktpolitik und Wohnbaupolitik sind somit entscheidend für die Ausgestaltung und die gesellschaftlichen Beiträge der Freiwilligenarbeit.

\section{Ebene der Gesellschaft}

Die integrative und inklusive Wirkung der Zivilgesellschaft, d.h. der NPOs, der Freiwilligen, der sozialen Bewegungen etc., folgt keinem Automatismus. So scheinen die bereits gesetzlich verankerten Einbürgerungserleichterung durch den Nachweis einer dreijährigen Freiwilligentätigkeit, in Anbetracht der Zugangsbarrieren tatsächlich ein valider Indikator für gelungene Integration zu sein. Gefördert wird Integration damit jedoch nicht. Davon ausgehend ist die Empfehlung, Freiwilliges Engagement ganz allgemein als Ausdruck gelungener gesamtgesellschaftlicher Integration, von gegenseitigem Vertrauen, von aktiver Bürgerschaft, u.v.m. zu betrachten und von funktionalistischen Anforderungen zu befreien. Denn die demokratischen und sozialen Werte des Dritten Sektors können nicht als gegeben angenommen, sondern müssen beständig erneuert und mit Leben erfüllt werden.

\section{(IV) 3. Fazit}

Obwohl die Freiwilligenarbeit in zahlreichen Feldern wie dem Sozialbereich, dem Gesundheitsbereich, der Katastrophenhilfe, der Rettungsdienste, dem Sport sowie bei vielen anderen freizeitorientierten und kulturellen Aktivitäten einen wichtigen Beitrag für die Gesellschaft leistet, trägt sie gleichzeitig auch zur Reproduktion ungleicher Lebensverhältnisse bei. Durch den sozial 
eingeschränkten Zugang ist der Erwerb von Kompetenzen, Sozialkontakten sowie gesundheitlichen Vorteilen als Ergebnis von Freiwilligem Engagement vorwiegend auf die bereits gut situierte Mittel- und Oberschicht beschränkt. Der sozial selektive Zugang zu den leitenden, mit Macht ausgestatteten Positionen, führt zu einer vielfach im Verborgenen stattfindenden weiteren Akkumulation von Macht, Prestige sowie bedeutsamen Sozialkontakten. Diese Formen der statusabhängigen Hierarchisierung finden sich in allen untersuchten Feldern der formellen Freiwilligenarbeit, vom Sport bis zum Sozialbereich und der Religion. Die Freiwilligenarbeit trägt so organisations- und feldübergreifend zu einer ungleichen Akkumulation von Prestige und Macht sowie ganz allgemein von sozialer Anerkennung bei. Der Beitrag der Freiwilligenarbeit zur Reproduktion sozialer Ungleichheit erfolgt dabei auf zweifache Art und Weise. Einerseits werden in der Freiwilligenarbeit Ungleichbehandlungen anderer Bereiche, wie sie beim Zugang zu sozialen Netzwerken und beruflichen Positionen zu finden sind, fortgeführt und verstärkt. Andererseits leistet die Freiwilligenarbeit durch die Verschleierung ihrer sozialen und symbolischen Profite einen originären Beitrag zur Reproduktion gesellschaftlicher Ungleichheit. Durch die Aura der „Freiwilligkeit“ und der „Uneigennützigkeit“ entzieht sich diese Form der Status- und Machtakkumulation bislang dem öffentlichen Blick und damit der expliziten Kritik. Die Aufgabe der Wissenschaft ist es, diese Mechanismen offen zu legen und mit ihren Forschungsergebnissen den kritischen Blick zu schärfen. 


\section{Literaturverzeichnis}

Adler, P. S., \& Kwon, S.-W. 2002. Social capital: Prospects For a New Concept. Academy of Management Review, 27(1): 17-40.

Alber, J., \& Lenarz, P. 2007. Wachsende soziale Ungleichheit in Europa - Die Lebensqualität unterer Einkommensschichten in der erweiterten Europäischen Union. Informationsdienst Soziale Indikatoren (ISI), 39: 1-5.

Alscher, M., Dathe, D., Priller, E., \& Speth, R. 2009a. Bericht zur Lage und zu den Perspektiven des bürgerlichen Engagements in Deutschland (Stand: Juni 2009, 1. Aufl. ed.). Berlin: Bundesministerium für Familie Senioren Frauen und Jugend.

Alscher, M., Dathe, D., Priller, E., \& Speth, R. 2009b. Nationaler und internationaler Stand der Engagementforschung. Monitor Engagement, $\mathrm{Nr}$ 1. Berlin: Bundesministerium für Familie Senioren Frauen und Jugend.

Amman, H. 2011. „Forschung Freiwilligkeit“ in der Schweiz. In E. Priller, M. Alscher, D. Dathe, \& R. Speth (Eds.), Zivilengagement. Herausforderungen für Gesellschaft, Politik und Wissenschaft: 227-248. Berlin: LIT Verlag.

Aner, K. 2007. Prekariat und Ehrenamt. In K. Aner, F. Karl, \& L. Rosenmayr (Eds.), Die neuen Alten - Retter des Sozialen? Wiesbaden: VS Verlag.

Aner, K., \& Hammerschmidt, P. 2008. Zivilgesellschaftlich produktiv altern. In M. Erlinghagen, \& K. Hank (Eds.), Produktives Altern und informelle Arbeit in modernen Gesellschaften. Theoretische Perspektiven und empirische Befunde. Wiesbaden: VS Verlag.

Anheier, H. K. 2013. Entwicklungen der internationalen Zivilgesellschaft. In R. Simsa, M. Meyer, \& C. Badelt (Eds.), Handbuch der Nonprofit Organisationen. Strukturen und Management: 77-88. Stuttgart: Schäffer-Poeschel.

Backes, G. M. 2005. Arbeit nach der Arbeit: Ehrenamtlichkeit und Freiwilligenarbeit älterer Menschen - Möglichkeiten und Illusionen. In W. Clemens (Ed.), Arbeit in späteren Lebensphasen. Sackgassen, Perspektiven, Visionen. Bern; Wien [u.a.]: Haupt.

Backes, G. M., \& Höltge, J. 2008. Überlegungen zur Bedeutung ehrenamtlichen Engagements im Alter. In M. Erlinghagen, \& K. Hank (Eds.), Produktives Altern und informelle Arbeit in modernen Gesellschaften. Theoretische Perspektiven und empirische Befunde. Wiesbaden: VS Verlag.

Backhaus, K. 2006. Multivariate Analysemethoden. Eine anwendungsorientierte Einführung (11., überarb. Aufl. ed.). Berlin u.a.: Springer.

Badelt, C. 1985. Politische Ökonomie der Freiwilligenarbeit. Theoretische Grundlagen und Anwendungen in der Sozialpolitik. Frankfurt am Main: Campus Verlag $\mathrm{GmbH}$. 
Badelt, C., \& More-Hollerweger, E. 2007. Ehrenamtliche Arbeit im Nonprofit Sektor. In Christoph Badelt, M. Meyer, \& R. Simsa (Eds.), Handbuch der Nonprofit Organisation. Strukturen und Management, Vol. 4. Auflage: 503-531. Stuttgart: Schäffer-Poeschel Verlag.

Baines, S., \& Hardill, I. 2008. 'At Least I can Do Something': The Work of Volunteering in a Cummunity Baset by Worklessness. Social Policy and Society, 7(3): 307-317.

Baur, J., \& Braun, S. (Eds.). 2003. Integrationsleistung von Sportvereinen als Freiwilligenorganisationen. Aachen: Meyer \& Meyer.

Baur, J., Braun, S., Burrmann, U., \& Nagel, M. 2003. Wer macht die Vereinspolitik in Sportvereinen? In J. Baur, \& S. Braun (Eds.), Integrationsleistung von Sportvereinen als Freiwilligenorganisationen: 268-302. Aachen: Meyer \& Meyer.

Beher, K. 2008. Die vergessene Elite - Führungskräfte in gemeinnützigen Organisationen. Weinheim u.a.: Juventa Verlag.

Bekkers, R. 2007. Intergenerational transmission of volunteering. Acta Sociologica, 50(2): 99-114.

Bekkers, R. 2011. Trust and Volunteering: Selection of Causation? Evidence From a 4 Year Panel Study. Political Behaviour: Published online: 9. April 2011.

Bekkers, R., \& Schuyt, T. 2008. And Who is Your Neighbor? Explaining Denominational Differences in Charitable Giving and Volunteering in the Netherlands. Review of Religious Research, 50(1): 74-96.

Berger, J. 2004. „Über den Ursprung der Ungleichheiten unter den Menschen“. Zur Vergangenheit und Gegenwart einer soziologischen Schlüsselfrage. Zeitschrift für Soziolgie, 33(5): 354-374.

Berger, P. A., Hank, K., \& Tölke, A. 2011. Reproduktion von Ungleichheit durch Arbeit und Familie. Wiesbaden: VS Verlag.

BMASK. 2009. Freiwilliges Engagement in Österreich. 1. Freiwilligenbericht. Wien: BMASK.

BMASK. 2013. Freiwilliges Engagement in Österreich. Bundesweite Bevölkerungsbefragung 2012. Studienbericht. Wien: BMASK.

Bödeker, S. 2012. Soziale Ungleichheit und politische Partizipation in Deutschland, OBS-Arbeitspapier 1. Frankfurt/Main: Otto-Brenner Stiftung.

Bohn, C. 1991. Habitus und Kontext. Ein kritischer Beitrag zur Sozialtheorie Bourdieus. Opladen: Westdeutscher Verlag.

Borgonovi, F. 2008. Divided We Stand, United We Fall: Religious Pluralism, Giving, and Volunteering. American Sociological Review, 73(1): 105-105-128.

Bourdieu, P. 1977. Entwurf einer Theorie der Praxis. Frankfurt am Main: Suhrkamp. 
Bourdieu, P. 1982. Die feinen Unterschiede: Kritik der gesellschaftlichen Urteilskraft (1. Aufl. ed.). Frankfurt am Main: Suhrkamp.

Bourdieu, P. 1983. Ökonomisches Kapital, kulturelles Kapital, soziales Kapital. In R. Kreckel (Ed.), Soziale Ungleichheiten. Göttingen: Schwartz.

Bourdieu, P. 1987. Sozialer Sinn. Kritik der theoretischen Vernunft. Frankfurt am Main: Suhrkamp.

Bourdieu, P. 1992a. Die verborgenen Mechanismen der Macht. Hamburg: VSA Verlag.

Bourdieu, P. 1992b. Rede und Antwort. Frankfurt am Main: Suhrkamp.

Bourdieu, P. 1998. Praktische Vernunft-Zur Theorie des Handelns (6. Aufl. ed.). Frankfurt am Main: Suhrkamp.

Bourdieu, P. 2000 (1973). Cultural reproduction and social reproduction. In R. Arum, \& I. R. Beattie (Eds.), The structure of schooling: Readings in the sociology of education: 56-69. Boston: McGraw Hill.

Bourdieu, P. 2001a. $<<$ Das $>>$ politische Feld. Zur Kritik der politischen Vernunft. Konstanz: UVK-Verl.-Ges.

Bourdieu, P. 2001b. Meditationen (1. Aufl. ed.). Frankfurt am Main: Suhrkamp.

Bourdieu, P. 2004. Soziologische Fragen (Dt. Erstausg., 4. Dr. ed.). Frankfurt am Main: Suhrkamp.

Bourdieu, P. 2005a. Die männliche Herrschaft (1. Aufl. ed.). Frankfurt am Main: Suhrkamp.

Bourdieu, P. 2005b. Die verborgenen Mechanismen der Macht. Hamburg: VSA Verlag.

Bowman, W. 2009. The Economic Value of Volunteers to Nonprofit Organizations. Nonprofit Management \& Leadership, 19(4): 491-506.

Brady, H. E. 2003. An Analytical Perspective on Participatory Inequality and Income Inequality, A paper for the Russell Sage Foundation Project on the "Social Dimensions of Inequality". Russell Sage Foundation Working Paper Series.

Brand, J. E. 2010. Civic Returns to Higher Education: A Note on Heterogeneous Effects. Social Forces, 89(2): 417-434.

Braun, S. 2001a. Bürgerschaftliches Engagement - Konjunktur und Ambivalenz einer gesellschaftspolitischen Debatte. Leviathan - Zeitschrift für Sozialwissenschaft, 29(1): 83-109.

Braun, S. 2001b. Putnam und Bourdieu und das soziale Kapital in Deutschland. Der rhetorische Kurswert einer sozialwissenschaftlichen Kategorie. LeviathanZeitschrift für Sozialwissenschaft, 29 (3): 337-354.

Braun, S. 2003a. "Social Capital" oder "Capital Social"? - Begriffspielerei als Gesellschaftsanalyse. Soziales Kapital als sozialwissenschaftliches und 
gesellschaftspolitisches Problem. In J. Baur, \& S. Braun (Eds.), Integrationsleistung von Sportvereinen als Freiwilligenorganisationen: 138-155. Aachen: Meyer \& Meyer.

Braun, S. 2003b. Sozialintegration, Systemintegration und Integration über sozialstrukturelle Bedingungen. In J. Baur, \& S. Braun (Eds.), Integrationsleistung von Sportvereinen als Freiwilligenorganisationen: 88-108. Aachen: Meyer \& Meyer.

Braun, S. 2003c. Sportvereine als freiwillige Vereinigungen im Dritten Sektor. Forschungsperspektiven. In J. Baur, \& S. Braun (Eds.), Integrationsleistung von Sportvereinen als Freiwilligenorganisationen: 667-694. Aachen: Meyer \& Meyer.

Braun, S. 2007. Freiwillige Vereinigungen als Katalysatoren von Sozialkapital? Ergebnisse einer repräsentativen Bevölkerungsbefragung in Deutschland. In J. Lüdicke, \& M. Diewald (Eds.), Soziale Netzwerke und soziale Ungleichheit. Zur Rolle von Sozialkapital in modernen Gesellschaften: 201-234. Wiesbaden: VS Verlag.

Brömme, N., \& Strasser, H. 2001. Gespaltene Bürgergesellschaft? Die ungleichen Folgen des Strukturwandels von Engagement und Partizipation. Aus Politik und Zeitgeschichte, B 25-26: 6-14.

Brown, E. 1999. Assessing the Value of Volunteer Activity. Nonprofit and Voluntary Sector Quarterly, 28(3): 3-17.

Brown, E., \& Ferris, M. J. 2007. Social Capital and Philanthropy: An Analysis of the Impact of Social Capital on Individual Giving and Volunteering. Nonprofit and Voluntary Sector Quarterly, 36(1): 85-99.

Brudney, J. L., \& Meijs, L. C. P. M. 2009. It Ain't Natural. Toward a New (Natural) Ressource Conceptualization for Volunteer Management. Nonprofit and Voluntary Sector Quarterly, 38(4): 564-581.

Brunie, A. 2009. Meaningful distinctions within a concept: Relational, collective, and generalized social capital. Social Science Research, 38: 251-265.

Budowski, M., \& Nollert, M. 2010. Soziale Ungleichheiten. Zürich: Seismo Verlag.

Bundesamt für Statistik (BFS). 2011. Freiwilligenarbeit in der Schweiz 2010. Neuchâtel: Bundesamt für Statistik (BFS).

Burr, J. A., Tavares, J., \& Mutschler, J. E. 2011. Volunteering and Hypertension Risk in Late Life. Journal of Aging and Health, 23(1): 24-51.

Caínzos, M., \& Voces, C. 2010. Class Inequalities in Political Participation and the 'Death of Class' Debate. International Sociology:, 25: 383-418.

Campbell, D. E. 2009. Civic Engagement and Education: An Empirical Test of the Sorting Model. American Journal of Political Science, 53(4): 771-786. 
Caputo, R. K. 2009. Religious capital and Intergenerational Transmission of Volunteering as Correaltes of Civic Engagement. Nonprofit and Voluntary Sector Quarterly, 38(6): 983-1002.

Carabain, C. L., \& Bekkers, R. 2011. Religious and secular volunteering: a comparison between immigrants and non-immigrants in the Netherlands Voluntary Sector Review, 2(1): 23-41.

Caro, F. G. 2008. Produktives Altern und ehrenamtliches Engagement in den USA. In M. Erlinghagen, \& K. Hank (Eds.), Produktives Altern und informelle Arbeit in modernen Gesellschaften. Theoretische Perspektiven und empirische Befunde. Wiesbaden: VS Verlag.

Cnaan, R. A., Handy, F., \& Wadsworth, M. 1996. Defining Who is a Volunteer: Conceptual and Empirical Considerations. Nonprofit and Voluntary Sector Quarterly, 25(3): 364-383.

Coffé, H., \& Geys, B. 2007. Toward an Empirical Characterization of Bridging and Bonding Social Capital. Nonprofit and Voluntary Sector Quarterly, 36(1).

Coleman, J. 1988. Social capital in the creation of human capital. American Journal of Sociology, 94 (Supplement): 95-120.

Corsten, M., Kauppert, M., \& Rosa, H. 2008. Quellen bürgerschaftlichen Engagements. Die biographische Entwicklung von Wir-Sinn und fokussierten Motiven. Wiesbaden: VS Verlag.

Czech, G. 2010. Sozialstrukturen im Österreichischen Roten Kreuz. Eine empirische Studie anhand vorliegender MitarbeiterInnendaten des Österreichischen Roten Kreuzes, Landesverband Niederösterreich. Unpublished Diplomarbeit, WU Wien, Wien.

Dathe, D. 2011. Engagement: Unbegrenzte Ressourcen für die Zivilgesellschaft? In E. Priller, M. Alscher, D. Dathe, \& R. Speth (Eds.), Zivilengagement. Herausforderungen für Gesellschaft, Politik und Wissenschaft: 41-56. Berlin: LIT Verlag.

Day, K. M., \& Devlin, R. A. 1996. Volunteerism and Crowding out: Canadian Econometric Evidence. The Canadian Journal of Economics, 29 (1): 37-53.

Day, K. M., \& Devlin, R. A. 1998. The payoff to work without pay: volunteer work as an investment in human capital. The Canadian Journal of Economics(31 (5)): 1179-1191.

Dekker, P. 2011. Freiwilliges Engagement und Engagmentforschung in den Niederlanden. In E. Priller, M. Alscher, D. Dathe, \& R. Speth (Eds.), Zivilengagement. Herausforderungen für Gesellschaft, Politik und Wissenschaft: 249-265. Berlin: LIT Verlag.

Dekker, P., \& van den Broek, A. 2005. Involvement in Voluntary Associations in North America and Western Europe: Trends and Correlates 1981-2000. Journal of Civil Society, 1(1): 45-59. 
Der Rat der Europäischen Union. 2010. Entscheidung des Rates vom 27. November 2009 über das Europäische Jahr der Freiwilligentätigkeit zur Förderung der aktiven Bürgerschaft (2011): Amtsblatt der Europäischen Union.

Deutschen Bundestag. Enquete-Kommission „Zukunft des Bürgerschaftlichen Engagements". 2002. Bericht. Bürgerschaftliches Engagement: auf dem Weg in eine zukunftsfähige Bürgergesellschaft. Opladen: Leske+Budrich.

DeVoe, S. E., \& Pfeffer, J. 2007. Hourly Payment and Volunteering: The Effect or Organizational practices and Decisions about Time Use. Academy of Management Journal, 50(4): 783 -798.

Diekmann, A. 2005. Empirische Sozialforschung. Grundlagen, Methoden, Anwendungen (Orig.-Ausg., 13. Aufl. ed.). Reinbek bei Hamburg: Rowohlt.

Diewald, M., \& Faist, T. 2011. Von Heterogänitäten zu Ungleichheiten: Soziale Mechanismen als Erklärungsansatz der Genese sozialer Ungleichheiten. Berliner Journal für Soziologie, 21: 91-114.

DiMaggio, P., \& Anheier, H. K. 1990. The Sociology of Nonprofit Organisations and Sectors. Annual Review of Sociology, 16: 137-159.

DiPrete, T. A., \& Eirich, G. M. 2006. Cumulative Advantage as a Mechanism for Inequality: A Review of Theoretical and Empirical Developements. Annual Review of Sociology, 32: 271-297.

Djupe, P. A., \& Grant, J. T. 2001. Religious Institutions and Political Participation in America. Journal for the Scientific Study of Religion, 40(2): 303-314.

Eagly, A. H. 2009. The His and Hers of Prosocial Behavior: An Examination of the Social Psychology of Gender. American Psychologist, 64(8): 644-658.

Egeraton, M. 2002. Higher education and civic engagement. British Journal of Sociology, 52 (4): 603-620.

Egerton, M., \& Mullan, K. 2008. Being a pretty good citizen: an analysis and monetary valuation of formal and informal voluntary work by gender and educational attainment. The British Journal of Socialogy, 59(1): 145-164.

Ehrenberg, A. 2011. Das Unbehagen in der Gesellschaft. Frankfurt am Main: Suhrkamp.

Ehrhardt, J. 2011. Ehrenamt. Formen, Dauer und kulturelle Grundlagen des Engagements. Frankfurt am Main: Campus Verlag GmbH.

Elshaug, C., \& Metzer, J. 2001. Personality attributes of volunteers and paid workers engaged in similar occupational tasks. The Journal of Social Psychology, 141(6): 752-752-763.

Elster, J. 2007. Explaining Social Behavior. More Nuts and Bolts for the Social Science. Ney York: Cambridge University Press.

Erlinghagen, M. 2000. Arbeitslosigkeit und ehrenamtliche Tätigkeit im Zeitverlauf. Eine Längsschnittanalyse der westdeutschen Stichprobe des 
Sozio-ökonomischen Panels (SOEP) für die Jahre 1992 und 1996. Kölner Zeitschrift für Soziologie und Sozialpsychologi, 52(2): 291-310.

Erlinghagen, M. 2003. Die individuellen Erträge ehrenamtlicher Arbeit. Zur sozio-ökonomischen Theorie unentgeltlicher, haushaltsextern organisierter Produktion Kölner Zeitschrift für Soziologie und Sozialpsychologie, 55: 737-757.

Erlinghagen, M. 2007. Die Beteiligung an ehrenamtlicher Arbeit und informeller Hilfe nach dem Renteneintritt. Analysen mit dem Sozio-Oekonomischen Panel (SOEP), Soeppapers on Multidisciplinary Panel Data Research, Vol. 27. Berlin: DIW

Erlinghagen, M. 2008. Ehrenamtliche Arbeit und informelle Hilfe nach dem Renteneintritt. In M. Erlinghagen, \& K. Hank (Eds.), Produktives Altern und informelle Arbeit in modernen Gesellschaften. Theoretische Perspektiven und empirische Befunde. Wiesbaden: VS Verlag.

Erlinghagen, M. 2010. Volunteering after Retirement. Evidence from German panel data. European Societies, 12(5): 603-625.

Erlinghagen, M., \& Hank, K. 2008. Produktives Altern und informelles Arbeiten in modernen Gesellschaften. Theoretische Perspektiven und empirischen Befunde. Wiesbaden: VS Verlag.

Erlinghagen, M., Hank, K., Lemke, A., \& Stuck, S. 2006. Produktives Potenzial jenseits der Erwerbsarbeit - Ehrenamtliches Engagement von Älteren in Deutschland und Europa. In B. Stiftung (Ed.), Älter werden - aktiv bleiben. Gütersloh: Verlag Bertelsmann Stiftung.

Esping-Andersen, G. 1990. The Three Worlds of Welfare Capitalism. Princeton, NJ: Princeton University Press.

European Values Study and GESIS Data Archive for the Social Sciences. 2011. EVS 1981-2008 Variable Report Longitudinal Data File. Köln: GESIS - Leibniz Institute for the Social Sciences.

European Volunteer Centre. 2006. Manifesto for Volunteering in Europe. Brussels: European Volunteer Centre.

Fiorillo, D. 2012. Volunteer Work an domain satisfaction: evidence from Italy. International Journal of Social Economics, 39(1/2): 97-124.

Fortuijn, J. D., \& van der Meer, M. 2006. Gender and voluntary work in late adulthood in rural communities in the Netherlands. GeoJournal, 65: 381-392.

Franzen, A., \& Freitag, M. 2007. Aktuelle Themen und Diskussionen der Sozialkapitalforschung. In A. Franzen, \& M. Freitag (Eds.), Sozialkapital. Grundlagen und Anwendungen. Kölner Zeitschrift für Soziologie und Sozialpsychologie, Sonderheft 47: 7-22. Wiesbaden: VS Verlag.

Fröhlich, G. 1994. Kapital, Habitus, Feld, Symbol. Grundbegriffe der Kulturtheorie bei Pierre Bourdieu. In G. Fröhlich, \& I. Mörth (Eds.), Das symbolische 
Kapital der Lebensstile: zur Kulturtheorie der Moderne nach Pierre Bourdieu. Frankfurt am Main: Campus Verlag GmbH.

Fuchs-Heinritz, W., \& König, A. 2005. Pierre Bourdieu. Eine Einführung. Konstanz: UVK Verlagsgesellschaft $\mathrm{mbH}$.

Fuhse, J. 2008. Netzwerke und soziale Ungleichheit. In C. Stegbauer (Ed.), Netzwerkanalyse und Netzwerktheorie: 79-90. Wiesbaden: VS-Verlag.

García-Mainar, I., \& Marcuello, C. 2007. Members, Volunteers, and Donors in Nonprofit Organizations in Spain. Nonprofit and Voluntary Sector Quarterly, 36(1): 100-120.

Gebauer, G., Braun, S., Suaud, C., \& Faure, J.-M. 1999. Die soziale Umwelt von Spitzensportlern - ein Vergleich des Spitzensports in Frankreich und Deutschland. Schorndorf: Hofmann.

Gensicke, T. 2008. Gemeinschaftsaktivitäten und freiwilliges Engagement älterer Menschen. In M. Erlinghagen, \& K. Hank (Eds.), Produktives Altern und informelle Arbeit in modernen Gesellschaften. Theoretische Perspektiven und empirische Befunde. Wiesbaden: VS Verlag.

Gensicke, T., \& Geiss, S. 2010. Hauptbericht des Freiwilligensurveys 2009. Zivilgesellschaft, soziales Kapital und freiwilliges Engagement in Deutschland 1999 - 2004 - 2009. Berlin: Bundesministerium für Familie, Senioren, Frauen und Jugend.

Gensicke, T., Picot, S., \& Geiss, S. 2006. Freiwilliges Engagement in Deutschland 1999-2004. In Bundesministerium für Familie Senioren Frauen und Jugend (Ed.). Wiesbaden: VS Verlag.

Gesthuizen, M., \& Scheepers, P. 2012. Educational Differences in Volunteering in Cross-National Perspective: Individual and Contextual Explanations. Nonprofit and Voluntary Sector Quarterly, 41(1): 58-81.

Goebel, J., Krause, P., \& Zähle, T. 2007. Dynamik von Einkommen und Armut in Ost- und West-Deutschland. Sozialer Fortschritt, 7-8: 200-207.

Granovetter, M. 1973. The Strength of Weak Ties. American Journal of Sociology, 78: $1360-1380$.

Granovetter, M. 2005. The Impact of Social Structure on Economic Outcomes. Journal of Economic Perspectives, 19 (1): 33-50.

Greenacre, M. 2007. Correspondence Analysis in Practice (Second Edition). Boca Raton: Taylor \& Francis Group, LLC.

Gross, N. 2009. A Pragmatist Theory of Social Mechanims. American Sociological Review, 74: 358-379.

Guy, M. E., \& Newman, M. A. 2004. Women's Jobs, Men's Jobs: Sex Segregation and Emotional Labor. Public Administration Review, 64 (3): 289-298. 
Hackl, F., Halla, M., \& Pruckner, G. J. 2007. Volunteering and Income - The Fallacy of the Good Samaritan? KYKLOS, 60 (1): 77-104.

Hackl, F., Halla, M., \& Pruckner, G. J. 2012. Volunteering and the state. Public Choice, 151: 465-495.

Haider, A., \& Schneider, U. 2009. Das Verhältnis von Freiwilligenarbeit und bezahlter Arbeit in Nonprofit Organistationen. In BMASK (Ed.), Freiwilliges Engagement in Österreich. 1. Freiwilligenbericht: 148-161. Wien: BMASK.

Handy, F., \& Cnaan, R. A. 2007. The Role of Social Anxiety in Volunteering. Nonprofit Management \& Leadership, 18 (1): 41-58.

Handy, F., Cnaan, R. A., Hustinx, L., Kang, C., Brudney, J. L., Haski-Leventhal, D., Holmes, K., Meijs, L. C. P. M., Pessi, A. B., Ranade, B., Yamauchi, N., \& Zrinscak, S. 2010 A Cross-Cultural Examination of Student Volunteering: Is It All About Résumé Building? Nonprofit and Voluntary Sector Quarterly, 39(3): 498-523.

Handy, F., \& Greenspan, I. 2009. Immigrant Volunteering. A Stepping Stone to Integration? Nonprofit and Voluntary Sector Quarterly, 38(6): 956-982.

Handy, F., \& Mook, L. 2011. Volunteering and Volunteers: Benefit-Cost Analyses. Research on Social Work Practice, 21: 412-420.

Hank, K., \& Erlinghagen, M. 2008. Produktives Altern und informelle Arbeit. In M. Erlinghagen, \& K. Hank (Eds.), Produktives Altern und informelle Arbeit in modernen Gesellschaften. Theoretische Perspektiven und empirische Befunde. Wiesbaden: VS Verlag.

Hank, K., \& Stuck, S. 2008. Ehrenamt, Netzwerkhilfe und Pflege in Europa. In M. Erlinghagen, \& K. Hank (Eds.), Produktives Altern und informelle Arbeit in modernen Gesellschaften. Theoretische Perspektiven und empirische Befunde. Wiesbaden: VS Verlag.

Hartmann-Tews, I., \& Combrink, C. 2006. Soziale Strukturen und Geschlechterordnung in den Führungsgremien von Sportverbänden. Spectrum der Sportwissenschaften, 18(2): 64-76.

Haski-Leventhal, D. 2009. Elderly Volunteering and Well-Being: A CrossEuropean Comparison Based on SHARE Data. VOLUNTAS: International Journal of Voluntary and Nonprofit Organizations, 20(4): 388-388-404.

Havens, J. J., O’Herlihy, M. A., \& Schervish, P. G. 2006. Charitable Giving: How Much, by Whom, to What, and How? In W. W. Powell, \& R. Steinberg (Eds.), The Nonprofit Sector: A Research Handbook, $2^{\text {nd }}$ edition ed.: 542-567. New Haven/London: Yale University Press.

Heimgartner, A. 2004. Ehrenamtliche bzw. freiwillige Arbeit in Einrichtungen Sozialer Arbeit. Frankfurt am Main; Wien u.a.: Lang. 
Heitzmann, K., Hofbauer, J., Mackerle-Bixa, S., \& Strunk, G. 2009. Where There's a Will, There's a Way? Civic Participation and Social Inequality. Journal of Civil Society, 5(3): 283-301.

Herzberg, F. 1987. One More Time: How Do You Motivate Employees? Haward Business Review: 109-120.

Hollerweger, E. 2001. Die Rolle von ehrenamtlicher Arbeit und Spenden im österreichischen Wohlfahrtsstaat., Working Paper Nr. 8/2001 des Instituts für Sozialpolitik. WU Wien.

Hooghe, M., \& Botterman, S. 2012. Urbanization, Community Size, and Population Density: Is There a Rural-Urban Divide in Partizipation in Voluntary Organizations or Social Network Formation? Nonprofit and Voluntary Sector Quarterly, 41(1): 120-144.

Houston, D. J. 2006. "Walking the Walk" of Public Service Motivation: Public Employees and Charitable Gifts of Time, Blood, and Money. Journal of Public Administration Research and Theory, 16 (1): 67-86.

Hovden, J. 2000. Gender and Leadership Selection Processes in Norwegian Sporting Organizations. International Review for the Sociology of Sport, 35 (1): 75-82.

Howard, M. M., \& Gilbert, L. 2008. A Cross-National Comparison of the Internal Effects of Participation in Voluntary Organizations. Political Studies, 56: 12-32.

Hradil, S. 2005. Soziale Ungleichheit in Deutschland (8. Aufl., Nachdr. ed.). Wiesbaden: VS Verlag.

Hradil, S. 2008. Soziale Ungleichheit, soziale Schichtung und Mobilität. In H. Korte, \& B. Schäfers (Eds.), Einführung in dei Hauptbegriffe der Soziologie: 213-234. Wiesbaden: VS Verlag.

Hradil, S. 2009. Lagen und Milieus. Sozialstrukturanalyse in einer fortgeschrittenen Gesellschaft. In H. Solga, J. Powell, \& P. A. Berger (Eds.), Soziale Ungleichheit. Klassische Texte zur Sozialstrukturanalyse: 281-311. Frankfurt am Main: Campus Verlag GmbH.

Hradil, S. 2013. Deutsche Verhältnisse. Eine Sozialkunde. Frankfurt am Main: Campus Verlag GmbH.

Hustinx, L. 2007. Brave New Volunteers? The Value of Paid and Unpaid Work for Flemish Red Cross Volunteers. VOLUNTAS: International Journal of Voluntary and Nonprofit Organizations, 18(1): 73-73-89.

Hustinx, L. 2010. Institutionally Individualized Volunteering: Towards a Late Modern Re-Construction. Journal of Civil Society, 6(2): 165-179.

Hustinx, L., Cnaan, R. A., \& Handy, F. 2010a. Navigating Theories of Volunteering: A Hybrid Map for a Complex Phenomenon. Journal for the Theory of Social Behaviour, 40 (4): 410-434. 
Hustinx, L., Handy, F., Cnaan, R. A., Brudney, J. L., Pessi, A. B., \& Yamauchi, N. 2010b. Social and Cultural Origins of Motivations to Volunteer. A Comparison of University Students in Six Countries. International Sociology, 25: 349-382.

Hustinx, L., \& Lammertyn, F. 2003. Collective and Reflexive Styles of Volunteering: A Sociological Modernization Perspective. VOLUNTAS: International Journal of Voluntary and Nonprofit Organizations, 14(2): 167-187.

Huth, S. 2002. Grundsatzthemen der Freiwilligenarbeit: Theorie und Praxis des sozialen Engagements und seine Bedeutung für ältere Menschen. Stuttgart; Marburg; Erfurt: Wiehl.

Huth, S. 2007. Bürgerschaftliches Engagement in Migrantenselbstorganisationen: integrationsfördernd oder -hemmend? Archiv für Wissenschaft und Praxis der sozialen Arbeit, 38 (3): 70-78.

Iglic, H. 2010. Voluntary Associations and Tolerance: An Ambiguous Relationship. American Behavioral Scientist, 53(5): 717-736.

Institute for Volunteering Research. 2004. Volunteering for All? Exploring the link between volunteering and social exclusion. London: Institute for Volunteering Research.

Janoski, T., Musick, M., \& Wilson, J. 1998. Beeing Volunteered? The Impact of Social Participation and Pro-Social Attitudes on Volunteering. Sociological Forum, 13(3): 495-519.

Janoski, T., \& Wilson, J. 1995. Pathways to Voluntarism: Family Socialization and Status Transmission Models. Social Forces, 74(1): 271-292.

Jung, J., \& Kwon, E. 2011. Trust Development in Volunteering: An Exploratory Study of Social Trust and Volunteering Activities in Korea. International Review of Public Administration, 16(1): 157-179.

Kazemipur, A. 2011. The Community Engagement of Immigrants in Host Societies: The Case of Canada. International Migration, 50(S1): e94-e116.

Kern, K. 2004. Sozialkapital, Netzwerke und Demokratie. In A. K. e. al (Ed.), Zivilgesellschaft und Sozialkapital. Herausforderungen politischer und sozialer Integration. Wiesbaden: VS Verlag.

Konietzka, D. 1995. Lebensstile im sozialstrukturellen Kontext. Ein theoretischer und empirischer Beitrag zur Analyse soziokultureller Ungleichheiten. Opladen: Westdeutscher Verlag.

Konrath, S., Fuhrel-Forbis, A., Lou, A., \& Brown, S. 2012. Motives for Volunteering Are Associated With Mortality Risk in Older Adults. Health Psychology, 31(1): 87-96.

Kreckel, R. 2004. Politische Soziologie der sozialen Ungleichheit (3., überarb. und erw. Aufl. ed.). Frankfurt am Main: Campus Verlag GmbH. 
Künemund, H., \& Schupp, J. 2007. Konjunktur des Ehrenamts - Diskurs und Empirie, Soeppapers on Multidisciplinary Panel Data Research Vol. 22. Berlin: DIW.

Lancee, B., \& Van de Werfhorst, H. G. 2012. Income inequality and participation: A comparison of 24 European countries. Social Science Research, 41: 1166-1178.

Le Roux, B., \& Rouanet, H. 2010. Multiple Correspondence Analysis. Thousand Oaks, CA: Sage.

Lemon, M., Palisi, B. J., \& Jacobson, P. E. J. 1972. Dominant Statuses and Involvement in Formal Voluntary Associations Nonprofit and Voluntary Sector Quarterly, 1(2): 30-42.

Lewis, M. A., \& Noguchi, E. 2006. The Female Corp of Volunteers: How Gender and Labor Supply Interact to Affect Civic Participation. Race, Gender \& Class, 13(3/4): 255-267.

Lichter, D. T., Shanahan, M. J., \& Gardner, E. L. 2002. Helping Others?: The Effects of Childhood Poverty and Family Instability on Prosocial Behavior. Youth Society, 34: 89-119.

Little, J. 1997. Constructions of Rural Women's Voluntary Work. Gender, Place \& Culture: A Journal of Feminist Geography, 4(2): 197-210.

Loveland, M. T., Sikkink, D., Myers, D. J., \& Radcliff, B. 2005. Private Prayer and Civic Involvement. Journal for the Scientific Study of Religion, 44(1): 1-14.

Lum, T. Y., \& Lightfoot, E. 2005. The Effects of Volunteering on the Physical and Mental Health of Older People. Research on Aging, 27(1): 31-55.

Machamer, P., Darden, L., \& Craver, C. F. 2000. Thinking about Mechanisms. Philosophy of Science, 67(1): 1-25.

Mackerle-Bixa, S., Meyer, M., \& Strunk, G. 2009. Membership and Participation: School of Democracy or Hideaway of Biedermeier? Journal of Civil Society, 5(3): 243-263.

Maier, F., \& Meyer, M. 2011. Managerialism and Beyond: Discourses of Civil Society Organization and Their Governance Implications. VOLUNTAS: International Journal of Voluntary and Nonprofit Organizations, 22 (4): 731-756.

Maier, F., Schober, C., Simsa, R., \& Millner, R. 2014. SROI as a Method for Evaluation Research: Understanding Merits and Limitations. VOLUNTAS: International Journal of Voluntary and Nonprofit Organizations: 1-26.

Marshall, G. A., \& Taniguchi, H. 2012. Good Jobs, Good Deeds: The GenderSpecific Influences of Job Characteristics on Volunteering. VOLUNTAS: International Journal of Voluntary and Nonprofit Organizations, 23: 213-235.

Marx, K., \& Engels, F. 1977 (1848). Manifest der Kommunistischen Partei In K. Marx, \& F. Engels (Eds.), Werke (Band 4): 459-493. Berlin: Dietz Verlag. 
Matsunaga, Y., Yamauchi, N., \& Okuyama, N. 2010. What Determines the Size of the Nonprofit Sector?: A Cross-Country Analysis of the Government Failure Theory. VOLUNTAS: International Journal of Voluntary and Nonprofit Organizations, 21: 180-201.

Mayrhofer, W., Meyer, M., \& Steyrer, J. 2007. Contextual Issues in the Study of Careers. In H. Gunz, \& M. Peiperl (Eds.), Handbook of Career Studies: 215240. Toronto: Sage.

McDougle, L., Handy, F., Konrath, S., \& Walk, M. 2013. Health Outcomes and Volunteering: the Moderation Role of Religiosity. Social Indicators Research.

McPherson, M., Smith-Lovin, L., \& Cook, J. M. 2001. Birds of a Feather: Homophily in Social Networks. Annual Review of Sociology, 27: 415-444.

Menchik, P. L., \& Weisbrod, B. A. 1987. Volunteer labor supply. Journal of Public Economics, 32(2): 159-183.

Merton, R. K. 2010. Der Matthäus-Effekt in der Wissenschaft, II. Kumulativer Vorteil und der Symbolismus des intellektuellen Eigentums. Berliner Journal für Soziologie, 20: 285-308.

Messner, M. A., \& Bozada-Deas, S. 2009. Separating the Men from the Moms: The Making of Adult Gender Segregation in Youth Sports. Gender \& Society, 23(49-71).

Meyer, M., Buber, R., \& Aghamanoukjan, A. 2013. In Search of Legitimacy: Managerialism and Legitimation in Civil Society Organizations. VOLUNTAS: International Journal of Voluntary and Nonprofit Organizations, 24 (1): 167-193.

Meyer, M., More-Hollerweger, E., \& Rameder, P. 2009. Freiwilligenarbeit im Alter. In E. Hanappi-Egger, \& P. Schnedlitz (Eds.), Ageing Society. Altern in der Stadt: Aktuelle Trends und ihre Bedeutung für die strategische Stadtentwicklung: 439-482. Wien: Facultas.

Meyer, M., \& Rameder, P. 2011. Freiwilligenarbeit im Kontext: individuelle, sozioökonomische und politische Einflussfaktoren. In KWG (Ed.), Freiwilligenarbeit. Symposium 2011. Wien: Manz'sche Verlags- und Universitätsbuchhandlung Gmbh.

Meyer, M., \& Simsa, R. 2013. NPOs: Abgrenzungen, Definitionen, Forschungszugänge. In R. Simsa, M. Meyer, \& C. Badelt (Eds.), Handbuch der Nonprofit Organisationen. Strukturen und Management: 3-14. Stuttgart: Schäfer-Poeschl.

Mitani, H. 2013. Influences of Resources and Subjective Dispositions on Formal and Informal Volunteering. VOLUNTAS: International Journal of Voluntary and Nonprofit Organizations, Published Online.

Mojza, E. J., Lorenz, C., Sonnentag, S., \& Binnewies, C. 2010. Daily Recovery Experiences: The Role of Volunteer Work During Leisure Time. Journal of Occupational Health Psychology, 15(1): 60-74. 
Mood, C. 2009. Logistic Regression: Why We Cannot Do What We Think We Can Do, and What We Can Do About It. European Sociological Review.

More-Hollerweger, E., \& Rameder, P. 2009. Freiwilliges Engagement und ältere Menschen. In BMASK (Ed.), Freiwilliges Engagement in Österreich. 1. Freiwilligenbericht: 120-136. Wien: BMASK.

More-Hollerweger, E., \& Rameder, P. 2013. Freiwilligenarbeit in NonprofitOrganisationen. In R. Simsa, M. Meyer, \& C. Badelt (Eds.), Handbuch der Nonprofit Organisationen. Strukturen und Management: 381-399. Stuttgart: Schäffer-Poeschel.

More-Hollerweger, E., Rameder, P., \& Spajcer, S. 2009a. Die Beziehung zwischen Freiwilligenarbeit und Erwerbsarbeit aus individueller Sicht. In BMASK (Ed.), Freiwilliges Engagement in Österreich. 1. Freiwilligenbericht: 74-89. Wien: BMASK.

More-Hollerweger, E., Spajcer, S., \& Eder, E. M. 2009b. Einführung - Definitionen und Abgrenzung von Freiwlligenarbeit. In BMASK (Ed.), Freiwilliges Engagement in Österreich. 1. Freiwilligenbericht: 1-12. Wien: BMASK.

More-Hollerweger, E., \& Sprajcer, S. 2009. Strukturen des Freiwilligensektors. In S. u. K. Bundesministerium für Arbeit (Ed.), Freiwilliges Engagement in Österreich: 30-48. Wien: BMSAK.

Moreno-Jime'nez, M. P., \& Hidalgo Villodres, M. C. 2010. Prediction of Burnout in Volunteers. Journal of Applied Social Psychology, 40(7): 1798-1818.

Morrow-Howell, N. 2010. Volunteering in Later Life: Research Frontiers. Journal of Gerontology: Social Science, 65B(4): 461-469.

Moxham, C., \& Boaden, R. 2007. The impact of performance measurement in the voluntary sector Identification of contextual and processual factors. International Journal of Operations \& Production Management, 27 (8): 826-845.

Mühlpfordt, S. 2006. Ehrenamt im Altersübergang. In S. Mühlpfordt, Richter, P. (Ed.), Ehrenamt und Erwerbsarbeit. München Rainer Hampp.

Munsch, C. 2010. Engagement und Diversität. Der Kontext von Dominanz und sozialer Ungleichheit am Beispiel Migration. Weinheim und München: Juventa Verlag.

Musick, M. A., \& Wilson, J. 2008. Volunteers. A Social Profile. Bloomington: Indiana University Press.

Mustillo, S., Wilson, J., \& Lynch, S. M. 2004. Legacy Volunteering: A Test of Two Theories of Intergenerational Transmission. Journal of Marriage and Family, 66: 530-541.

Mutchler, J. E., Burr, J. A., \& Caro, F. G. 2003. From Paid Worker to Volunteer: Leaving the Paid Workforce and Volunteering in Later Life. Social Forces, 81 (4): 1267-1293. 
Nagel, M. 2003a. Die soziale Ordnung freiwilliger Vereinigungen. In J. Baur, \& S. Braun (Eds.), Integrationsleistung von Sportvereinen als Freiwilligenorganisationen: 464-486. Aachen: Meyer \& Meyer.

Nagel, M. 2003b. Die soziale Zusammensetzung der Sportvereinsmitglieder. In J. Baur, \& S. Braun (Eds.), Integrationsleistung von Sportvereinen als Freiwilligenorganisationen: 409-448. Aachen: Meyer \& Meyer.

Nagel, M. 2003c. Sportvereine im Spannungsfeld zwischen sozialer Offenheit und sozialer Geschlossenheit. In J. Baur, \& S. Braun (Eds.), Integrationsleistung von Sportvereinen als Freiwilligenorganisationen: 449-463. Aachen: Meyer \& Meyer.

Nassehi, A. 2008. Soziologie. Zehn einführende Vorlesungen. Wiesbaden: VS Verlag.

Nesbit, R., \& Gazley, B. 2012. Patterns of Volunteer Activity in Professional Associations and Societies. VOLUNTAS: International Journal of Voluntary and Nonprofit Organizations, 23: 558-583.

Neumayr, M., \& More-Hollerweger, E. 2009. Freiwilliges Engagement und Gender. In BMASK (Ed.), Freiwilliges Engagement in Österreich. 1. Freiwilligenbericht: 90-103. Wien: BMASK.

Nichols, G., \& Ralston, R. 2011. Social Inclusion through Volunteering: The Legacy Potential of the 2012 Olympic Games. Sociology, 45(5): 900-914.

Oesterle, S., Johnson, M. K., \& Mortimer, J. T. 2004. Volunteerism during the Transition to Adulthood: A Life Course Perspective. Social Forces, 82(3): 1123-1149.

Olk, T. 2002. Modernisierung des Engagements im Alter - Vom Ehrenamt zum bürgerschaftlichen Engagement?: eine Veröffentlichung der Bundesarbeitsgemeinschaft Seniorenbüros $(\mathrm{BaS})$, Bonn, Institut für Soziale Infrastruktur (ISIS), Frankfurt am Main (HG), Stuttgart-Marburg-Erfurt.

Ostrower, F. 2002. Trustees of Culture. Power, Wealth, and Status on Elite Arts Boards. Chicago, London: The University of Chicago.

Pampel, F. C. 2000. Logistic Regression. A Primer. Thousand Oaks, CA: Sage.

Parboteeah, K. P., Cullen, J. B., \& Lim, L. 2004. Formal volunteering: a cross national test. Journal of World Business, 39: 431-441.

Park, C.-u., \& Subramanian, S. V. 2012. Voluntary Association Membership and Social Cleavages: A Micro-Macro Link in Generalized Trust. Social Forces, 90(4): 1183-1205.

Pearce, J. L. 1993. Volunteers the organizational behavior of unpaid workers (1. publ. ed.). London u.a.: Routledge.

Penner, L. A., Dovidio, J. F., Piliavin, J. A., \& Schroeder, D. A. 2005. Prosocial Behavior: Multilevel Perspectives. Annual Review of Psychology, 56: 14.11-14.28. 
Peter, S., \& Drobnič, S. 2013. Women and their memberships: Gender gap in relational dimension of social inequality. Research in Social Stratification and Mobility, 31: 34-48.

Pikkety, T. 2014. Capital in the Twenty-First Century: Harvard University Press.

Placke, G., \& Riess, B. 2006. Bürgerschaftliches Engagement in der zweiten Lebenshälfte: Freiwillige Tätigkeiten in Wechselwirkung zur Erwerbsarbeit. In Bertelsmann-Stiftung (Ed.), Älter werden - aktiv bleiben. Gütersloh: Verlag Bertelsmann Stiftung.

Plagnol, A. C., \& Huppert, F. A. 2010. Happy to Help? Exploring the Factors Associated with Variations in Rates of Volunteering Across Europe. Social Indicators Research, 97(2): 157-157-176.

Popielarz, P. A. 1999. (In)Voluntary Association. A Multilevel Analysis of Gender Segregation in Voluntary Organisations. Gender \& Society, 13(2): 234-250.

Prein, G., Sass, E., \& Züchner, I. 2009. Lernen im freiwilligen Engagement und gesellschaftliche Partizipation. Ein empirischer Versuch zur Erklärung politischen Handelns. Zeitschrift für Erziehungswissenschaften, 12: 529-547.

Priller, E. 2010. Stichwort: Vom Ehrenamt zum zivilgesellschaftlichen Engagement. Zeitschrift für Erziehungswissenschaften, 13: 195-213.

Priller, E. 2011. Dynamik, Struktur und Wandel der Engagementforschung: Rückblick, Tendenzen und Anforderungen. In E. Priller, M. Alscher, D. Dathe, \& R. Speth (Eds.), Zivilengagement. Herausforderungen für Gesellschaft, Politik und Wissenschaft: 11-40. Berlin: LIT Verlag.

Putnam, R. D. 1993. Making democracy work. Civic traditions in modern Italy. Princeton, NJ: Princeton Univ. Press.

Putnam, R. D. 1995. Bowling Alone: America’s Declining Social Capital. Journal of Democracy, 6: 65-78.

Putnam, R. D. 2000. Bowling alone. The collapse and revival of American community. New York, NY [u.a.]: Simon \& Schuster.

Radtke, S. 2007. Ehrenamtliche Führungskräfte im organisierten Sport. Biographische Zusammenhänge und Motivationen. Hamburg: Dr. Kovac.

Rameder, P., \& More-Hollerweger, E. 2009. Beteiligung am freiwilligen Engagement in Österreich. In BMASK (Ed.), Freiwilliges Engagement in Österreich. 1. Freiwilligenbericht: 49-73. Wien: BMASK.

Randle, M., \& Dolnicar, S. 2011. Self-congruity and volunteering: a multiorganisation comparison. European Journal of Marketing, 45(5): 739-739-758.

Rehberg, K.-S. 2011. „Klassengesellschaftlichkeit“ nach dem Ende der Klassengesellschaft? Berliner Journal für Soziologie, 21: 7-21. 
Reinprecht, C. 2009. Freiwilliges Engagement und Migrantinnen/Migranten. In BMASK (Ed.), Freiwilliges Engagement in Österreich. 1. Freiwilligenbericht: 137-147. Wien: BMASK.

Reskin, B. F. 2003. Including Mechanisms in our Models of ascriptive inequality. American Sociological Review, 68: 1-21.

Rigney, D. 2010. The Matthew effect. how advantage begets further advantage. New York: Columbia University Press.

Rochester, C., Paine, A. E., Howlett, S., \& Zimmeck, M. 2010. Volunteering and Society in the 21st Century. Hampshire: Palgrave Macmillan.

Roth, R. 2004. Die dunklen Seiten der Zivilgesellschaft. Grenzen einer Zivilgesellschaftlichen Fundierung von Demokratie. In A. Klein, K. Kern, B. Geißel, \& M. Berger (Eds.), Zivilgesellschaft und Sozialkapital. Herausforderungen politischer und sozialer Integration. Wiesbaden: VS Verlag.

Rotolo, T., \& Wilson, J. 2007. Sex Segregation in Voluntary Work. The Sociological Quarterly, 48: 559-585.

Rotolo, T., \& Wilson, J. 2012. State-Level Differences in Volunteerism in the United States: Research Based on Demographic, Institutional, and Cultural Macrolevel Theories. Nonprofit and Voluntary Sector Quarterly, 41(3): 452-473.

Rotolo, T., Wilson, J., \& Hughes, M. E. 2010. Homeownership and Volunteering: An Alternative Approach to Studying Social Inequality and Civic Engagement. Sociological Forum, 25(3): 570-587.

Ruiter, S., \& De Graaf, N. D. 2009. Socio-economic Payoffs of Voluntary Association Involvement: A Dutch Life Course Study. European Sociological Review, 25 (4): 425-442.

Ruzza, C. 2009. Populism and euroscepticism: Towards uncivil society? Policy and Society, 28: 87-98.

Salamon, L. M., \& Anheier, H. K. 1998. Social origins of civil society: Explaining the nonprofit sector cross-nationally. VOLUNTAS: International Journal of Voluntary and Nonprofit Organizations, 9(3): 213-248.

Salamon, L. M., Hems, L. C., \& Chinnock, K. 2000. The Nonprofit Sector: For What and for Whom?, Working Papers of the Johns Hopkins Comparative Nonprofit Sector Project.

Salamon, L. M., Sokolowski, S. W., \& Haddock, A. M. 2011. Measuring the Economic Value of Voluntary Work Globally: Concepts, Estimates, and a Roadmap to the Future. Annals of Public and Cooperative Economics 82(3): 217-252.

Salamon, L. M., \& Sokolowski, W. 2001. Volunteering in Cross-National Perspective: Evidence From 24 Countries, Working Papers of the Johns Hopkins Comparative Nonprofit Sector Project. 
Schell, U. 2009. Frauen in ehrenamtlichen Leitungsfunktionen. Symbolische, biographische und institutionell-strukturelle Eckpunkte. Dargestellt am BDKJ-Diözesanverband Augsburg und seinen Untergliederung. Unpublished Monographie, Universität Wien.

Schimpl-Neimanns, B. 2004. Zur Umsetzung des Internationalen Sozioökonomischen Index des beruflichen Status (ISEI). ZUMA-Nachrichten, 54: 154-170.

Schlesinger, T., \& Nagel, S. 2013. Who will volunteer? Analysing individual and structural factors of volunteering in Swiss sports clubs. European Journal of Sport Science, 13(6): 707-715.

Schneider, J. A. 2009. Organizational Social Capital and Nonprofits. Nonprofit and Voluntary Sector Quarterly, 38(4): 643-662.

Schnittker, J., \& Behrman, J. R. 2012. Learning to do well or learning to do good? Estimating the effects of schooling on civic engagement, social cohesion, and labor market outcomes in the presence of endowments. Social Science Research, 41(2): 306-320.

Schroeter, K. R. 2006. Altern und bürgerschaftliches Engagement. Aspekte der Vergemeinschaftung und Vergesellschaftung in der Lebensphase Alter. Wiesbaden: VS Verlag.

Schwinn, T. 2007. Soziale Ungleichheit. Bielefeld: Transcript Verlag.

Schwinn, T. 2008. Zur Analyse multidimensionaler Ungleichheiten. Österreichische Zeitschrift für Soziologie, 33 (1): 20-42.

Seippel, Ø. 2005. Sport, Civil Society and Social Integration: The Case of Norwegian Voluntary Sport Organizations. Journal of Civil Society, 1(3): 247-265.

Seubert, S. 2009. Das Konzept des Sozialkapitals. Eine demokratietheoretische Analyse. Frankfurt am Main: Campus Verlag GmbH.

Smith, D. H. 1975. Voluntary Action and Voluntary Groups. Annual Review of Sociology, 1: 247-270.

Smith, D. H. 1994. Determinants of Voluntary Association Participation and Volunteering: A Literature Review. Nonprofit and Voluntary Sector Quarterly, 23: 243-263.

Snyder, M., \& Omoto, A. M. 2008. Volunteerism: Social Issues Perspectives and Social Policy Implications. Social Issues and Policy Review, 2(1): 1-36.

Solga, H., Berger, P. A., \& Powell, J. 2009. Soziale Ungleichheit - Kein Schnee von gestern! Eine Einführung. In H. Solga, J. Powell, \& P. A. Berger (Eds.), Soziale Ungleichheit. Klassische Texte zur Sozialstrukturanalyse: 11-45. Frankfurt am Main: Campus Verlag GmbH.

Solt, F. 2008. Economic Inequality and Democratic Political Engagement. American Journal of Political Science, 52(1): 48-60. 
Son, S., \& Wilson, J. 2010. Genetic variation in volunteerism. The Sociological Quarterly, 51: 46-64.

Spence, M. 1973. Job Market Signaling. The Quarterly Journal of Economics, 87 (3): 355-374.

Stadelmann-Steffen, I. 2011. Social Volunteering in Welfare States: Where Crowding out Should Occur. Political Studies, 59: 135-155.

Stadelmann-Steffen, I., \& Freitag, M. 2011. Making Civil Society Work: Model of Democracy and Their Impact on Civic Engagement. Nonprofit and Voluntary Sector Quarterly, 40(3): 526-551.

Stadelmann-Steffen, I., Freitag, M., \& Bühlmann, M. 2007. Freiwilligen-Monitor Schweiz 2007. Zürich: Seismo Verlag.

Stadelmann-Steffen, I., Traunmüller, R., Gundelach, B., \& Freitag, M. 2010. Freiwilligen-Monitor Schweiz 2010. Zürich: Seismo Verlag.

Staines, G. 1980. Spillover versus Compensation: A Review of the Literature on the Relationship between Work and Nonwork. Human Relations, 33: 111-129.

Stater, K. J. 2010. How Permeable is the Nonprofit Sector? Linking Resources, Demand, and Government Provision to the Distribution of Organizations Across Nonprofit Mission-Based Fields. Nonprofit and Voluntary Sector Quarterly, 39(4): 674-695.

Statistik Austria. 2007. Struktur und Volumen der Freiwilligenarbeit in Österreich. Wien: Bundesministerium für Soziales und Konsumentenschutz.

Statistik Austria. 2008. Struktur und Volumen der Freiwilligenarbeit in Österreich. Bericht im Auftrag des Bundesministeriums für Soziales, Gesundheit und Konsumentenschutz, Vol. 2010. Wien.

Stiglitz, J. 2012. Der Preis der Ungleichheit: Wie die Spaltung der Gesellschaft unsere Zukunft bedroht München: Siedler Verlag.

Stolle, D., \& Hooghe, M. 2005. Inaccurate, exceptional, one-sided or irrelevant? The debate about the alleged decline of social capital and civic engagement in western societies. British Journal of Political Science, 35 (1): 149-167.

Strauß, S. 2009. Ehrenamt in Deutschland und Großbritannien - Sprungbrett zurück auf den Arbeitsmarkt?. Kölner Zeitschrift für Soziologie und Sozialpsychologie, 61: 647-670.

Studer, S., \& von Schnurbein, G. 2012. Organizational Factors Affecting Volunteers: A Literature Review on Volunteer Coordination. VOLUNTAS: International Journal of Voluntary and Nonprofit Organizations: 1-38.

Suanet, B., Groenou, M. B. v., \& Braam, A. W. 2009. Changes in volunteering among young old in the Netherlands between 1992 amd 2002: the impact of religion, age-norms, and intergenerational transmission. European Journal of Ageing, 6: 157-165. 
Sundeen, R. A., Garcia, C., \& Raskoff, S. A. 2009. Ethnicity, Acculturation, and Volunteering to Organizations: A Comparison of African Americans, Asians, Hispanics, and Whites. Nonprofit and Voluntary Sector Quarterly, 38(6): 929-955.

Sundeen, R. A., \& Raskoff, S. A. 2000. Ports of Entry and Obstacles: Teenagers' Access to Volunteer Activities. Nonprofit Management \& Leadership, 11(2): 179-197.

Syvertsen, A. K., Wray-Lake, L., Flanagan, C. A., Osgood, D. W., \& Briddell, L. 2011. Thirty Year Trends in U.S. Adolescents' Civic Engagement: A Story of Changing Participation and Educational Differences. Journal of Research on Adolescence, 21(3): 586-594.

Tang, F. 2006. What Resources Are Needed for Volunteerism? A Life Course Perspective. The Journal of Applied Gerontology, 25 (5): 375-390.

Tang, F. 2008. Socioeconomic Disparities in Voluntary Organization Involvement Among Older Adults. Nonprofit and Voluntary Sector Quarterly, 37/1 March 2008: 57-75.

Taniguchi, H. 2006. Men's and Women's Volunteering: Gender Differences in the Effects of Employment and Family Characteristics. Nonprofit and Voluntary Sector Quarterly, 35(1): 83-101.

Taniguchi, H., \& Thomas, L. D. 2011. The Influences of Religious Attitudes on Volunteering. VOLUNTAS: International Journal of Voluntary and Nonprofit Organizations, 22(2): 335-335-355.

Themudo, N. S. 2009. Gender and the Nonprofit Sector. Nonprofit and Voluntary Sector Quarterly, 38(4): 663-683.

Therborn, G. (Ed.). 2006. Inequalities of the world. New theoretical frameworks, multiple empirical approaches (1. publ. ed.). London [u.a.]: Verso.

Tiehen, L. 2000. Has Working More Caused Married Women to Volunteer Less? Evidence From Time Diary Data, 1965 to 1993. Nonprofit and Voluntary Sector Quarterly, 29 (4): 505-529.

Tschirhart, M. 2006. Nonprofit Membership Associations. In W. W. Powell, \& R. Steinberg (Eds.), The Nonprofit Sector: A Research Handbook, $2^{\text {nd }}$ edition ed.: 523-541. New Haven/London: Yale University Press.

United Nations - Department of Economic and Social Affairs Statistics Division. 2003. Handbook on Non-Profit Institutions in the System of National Accounts. ST/ESA/STAT/SER.F/91. New York.

United Nations. 2002. Resolution 56/38. Recommendations on support for volunteering.

United Nations. 2009. Resolution 63/153. Follow-up to the implementation of the International Year of Volunteers. In G. Assembly (Ed.). 
Uslaner, E. M., \& Brown, M. 2008. Inequality, Trust and Civic Engagement. American Politics Research, 33: 868-894.

van Deth, J. W. 2004. Soziale Partizipation, Deutschland in Europa. Ergebnisse des European Social Survey 2002-2003: 296-315. Wiesbanden: VS Verlag.

van Deth, J. W. 2010. Participation in Voluntary Associations and Tolerance: Dark Shades in a Sunny World? American Behavioral Scientist, 53(5): 640-656.

van Deth, J. W., Montero, J. R., \& Westholm, A. (Eds.). 2007. Citizenship and Involvement in European Democracies. London: Routledge.

van Houten, J. M. A., Gesthuizen, M., \& Wolbers, M. H. J. 2013. Intergenerational transmission of occupational status: The role of voluntary association membership as an emerging compensatory strategy of reproduction. Research in Social Stratification and Mobility, 33: 13-26.

van Ingen, E. 2009. Let's Come Together and Unite. Studies of the Changing Character of Voluntary Association Participation. Tilburg: Tilburg University.

van Ingen, E., \& Dekker, P. 2010. Changes in the Determinants of Volunteering: Participation and Time Investment Between 1975 and 2005 in the Netherlands. Nonprofit and Voluntary Sector Quarterly, 40(4): 682-702.

van Ingen, E., \& Kalmihn, M. 2010. Does Voluntary Association Participation Boost Social Resources. Social Science Quarterly, 91(2): 493-510.

van Tienen, M., Scheepers, P., Reitsma, J., \& Schilderman, H. 2011. The Role of Religiosity for Formal and Informal Volunteering in the Netherlands. VOLUNTAS: International Journal of Voluntary and Nonprofit Organizations, 22(3): 365-365-389.

Verba, S., Schlozman, K. L., \& Brady, H. E. 1995. Voice and Equality: Civic Voluntarism in American Politics. Cambridge, MA: Harvard University Press.

Verba, S., Schlozman, K. L., \& Brady, H. E. 2002. Voice and equality. Civic voluntarism in American politics (4 ed.). Cambridge, Mass. u.a.: Harvard Univ. Press.

Vermeer, P., \& Scheepers, P. 2012. Religious Socialisation and Non-Religious Volunteering: A Dutch Panel Study. VOLUNTAS: International Journal of Voluntary and Nonprofit Organizations, 23: 940-958.

Vogt, L. 2005. Das Kapital der Bürger. Theorie und Praxis zivilgesellschaftlichen Engagements. Frankfurt am Main: Campus Verlag GmbH.

Wahrendorf, M., \& Siegrist, J. 2008. Soziale Produktivität und Wohlbefinden im höheren Lebensalter. In M. Erlinghagen, \& K. Hank (Eds.), Produktives Altern und informelle Arbeit in modernen Gesellschaften. Theoretische Perspektiven und empirische Befunde. Wiesbaden: VS Verlag. 
Wallace, C., \& Pichler, F. 2009. More Participation, Happier Society? A Comparative Study of Civil Society and Quality of Life. Social Indicators Research, 93: 255-274.

Walter, F. 2012. Von Teilhabe ausgeschlossen? Soziale Ungleichheit und politischen Partizipation in Österreich. Kurswechsel, 3/2012: 40-48.

Webb, J. N., \& Abzug, R. 2008. Do Occupational Group Members Vary in Volunteering Activity?. Nonprofit and Voluntary Sector Quarterly, 37(4): 689-708.

Weber, M. 1921/1980. Wirtschaft und Gesellschaft. Grundriss einer verstehenden Soziologie. Tübingen: J.C.B. Mohr (Paul Siebeck).

Weiß, A. 2004. Unterschiede die einen Unterschied machen. Klassenlagen in den Theorien von Pierre Bourdieu und Niklas Luhmann. In A. Nassehi, \& G. Nollmann (Eds.), Bourdieu und Luhmann. Ein Theorievergleich: 208-232. Frankfurt am Main: Suhrkamp.

Wiepking, P., \& Bekkers, R. 2012. Who Gives? A Literature Review of Predictors of Charitable Giving II - Gender, Marital Status, Income and Wealth. Voluntary Sector Review, 3(2): 217-246.

Wilkinson, R., \& Pickett, K. 2009. The Spirit Level. Why More Equal Societies Almost Do Better. London: Penguin Group.

Wilson, J. 2000. Volunteering. Annual Review of Sociology, 26: 215-240.

Wilson, J. 2012. Volunteerism Research: A Review Essay. Nonprofit and Voluntary Sector Quarterly, 41(2): 176-212.

Wilson, J., \& Musick, M. A. 1997a. Who cares? Toward an integrated theory of volunteer work. American Sociological Review, 62(5): 694-713.

Wilson, J., \& Musick, M. A. 1997b. Work and Volunteering. The long arm of the Job. Social Forces, 76(1): 251-272.

Wilson, J., \& Musick, M. A. 1999. The Effect of Volunteering on the Volunteer. Law and Contemporary Problems, 62(4): 141-168.

Wilson, J., \& Musick, M. A. 2000. Race and formal Volunteering. The Differential Effects of Class and Religion. Social Forces, 78(4): 1539-1570.

Winker, G., \& Degele, N. 2009. Intersektionalität. Zur Analyse sozialer Ungleichheit. Bielefeld: transkript Verlag.

Winkler, J. 1995. Lebensstil und Sport. Der Sport als 'stilistische Möglichkeit' in der Symbolisierung von Lebensführung. In J. Winkler, \& K. Weis (Eds.), Soziologie des Sports: Theorieansätze, Forschungsergebnisse, und Forschungsperspektiven. Opladen: Westdeutscher Verlag.

Wolozin, H. 1975. The Economic Role and Value of Volunteer Work in the United States: an Exploratory Study. Nonprofit and Voluntary Sector Quarterly, 4(1-2): 23-42 
Wouters, G. 2005. Zur Identitätsrelevanz von freiwilligem Engagement im dritten Lebensalter: Anzeichen einer Tätigkeitsgesellschaft. Herbolzheim: Centaurus-Verl.

Zimmer, A. 2009. Bürgerschaftliches Engagement - Thema von Lehre und Forschung? In I. Bode, A. Evers, \& A. Klein (Eds.), Bürgergesellschaft als Projekt. Eine Bestandsaufnahme zu Entwicklung und Förderung zivilgesellschaftlicher Potenziale in Deutschland: 80-100. Wiesbaden: VS Verlag. 



\section{Anhang}

\section{Anhang 1: Theoretische Perspektiven}

Tabelle 34: Inequality Mechanism and their Interactive Dynamics

\begin{tabular}{|c|c|c|}
\hline Mechanism & Direct agency & $\begin{array}{l}\text { Dynamics } \\
\text { Systemic dynamics }\end{array}$ \\
\hline Distantiation & $\begin{array}{l}\text { Running ahead/falling } \\
\text { behind } \\
\text { Outcompeting } \\
\text { Social psychology of } \\
\text { success/failure }\end{array}$ & $\begin{array}{l}\text { Reward structuration and normation, } \\
\text { e.g. 'Winner takes all', 'Matthew effect', } \\
\text { 'star' system } \\
\text { Returns to scale } \\
\text { Opportunity structuration }\end{array}$ \\
\hline Exclusion & $\begin{array}{l}\text { Closure, Hindering } \\
\text { Opportunity hoarding } \\
\text { Discrimination, } \\
\text { monopolization }\end{array}$ & $\begin{array}{l}\text { Membership boundaries, entry } \\
\text { thresholds } \\
\text { Cumulation of advantages } \\
\text { Stigmatization } \\
\text { Citizenship/property rights }\end{array}$ \\
\hline Hierarchization & $\begin{array}{l}\text { Super-subordination } \\
\text { Patron-client relation } \\
\text { Put-Down/deference }\end{array}$ & $\begin{array}{l}\text { Organizational ladder, status/authority } \\
\text { distance } \\
\text { Hierarchy of family roles } \\
\text { Systemic center and peripheries } \\
\text { Ethnic/racial/gendered hierarchies } \\
\text { Generalizations of super-inferiority }\end{array}$ \\
\hline Exploitation & $\begin{array}{l}\text { Extraction } \\
\text { Utilization } \\
\text { Abuse }\end{array}$ & $\begin{array}{l}\text { Polarized power relations } \\
\text { Asymmetric dependence } \\
\text { Tributary systems }\end{array}$ \\
\hline
\end{tabular}

Quelle: Therborn (2006: 14).

Tabelle 35: Equality Mechanisms

\begin{tabular}{ll}
\hline Catching up & $\begin{array}{l}\text { Field-evening, compensatory capacitation, } \\
\text { 'affirmative action', new opportunity openings }\end{array}$ \\
Inclusion & Entitlement, migration, human rights \\
Organizational/institutional & Empowerment, democratization, unionization, \\
flattening & user rights \\
Redistribution & Taxation, social policy \\
\hline
\end{tabular}

Quelle: Therborn (2006: 14). 


\section{Anhang 2: Empirische Analysen}

Tabelle 36: Beteiligungsquoten und Beteiligungsstruktur

\begin{tabular}{|c|c|c|c|c|}
\hline & & $\begin{array}{c}\text { Mikrozensus - } \\
\text { Zusatzerhebung } \\
\text { „Freiwilligenarbeit“ }\end{array}$ & $\begin{array}{c}\text { Beteiligungs- } \\
\text { quoten (\%) } \\
\text { an formeller } \\
\text { Freiwilligenarbeit } \\
(\mathrm{N}=11.657)\end{array}$ & $\begin{array}{l}\text { Beteiligungs- } \\
\text { struktur (\%) in } \\
\text { der formellen } \\
\text { Freiwilligenarbeit } \\
\quad(\mathrm{N}=4.818)\end{array}$ \\
\hline \multirow{2}{*}{ Geschlecht } & Männlich & 5.502 & 49,0 & 56,0 \\
\hline & Weiblich & 6.155 & 34,4 & 44,0 \\
\hline \multirow{4}{*}{ Alter } & 15-29 Jahre & 2.193 & 41,8 & 19,0 \\
\hline & 30-49 Jahre & 5.085 & 44,1 & 46,7 \\
\hline & 50-64 Jahre & 2.782 & 41,3 & 23,8 \\
\hline & $65+$ Jahre & 1.597 & 31,6 & 10,5 \\
\hline \multirow{2}{*}{$\begin{array}{l}\text { Geburtsland } \\
\text { Österreich }\end{array}$} & Österreich & 10.723 & 42,5 & 94,6 \\
\hline & Nicht-Österreich & 934 & 27,9 & 5,4 \\
\hline \multirow{6}{*}{$\begin{array}{l}\text { Höchste abgeschlos- } \\
\text { sene Ausbildung }\end{array}$} & $\begin{array}{l}\text { Pflichtschule/keine } \\
\text { Pflichtschule }\end{array}$ & 2.326 & 31,0 & 17,9 \\
\hline & $\begin{array}{l}\text { Lehrabschluss } \\
\text { (Berufsschule) }\end{array}$ & 4.305 & 40,1 & 35,9 \\
\hline & $\begin{array}{l}\text { Berufsbild. mittlere } \\
\text { Schule }\end{array}$ & 1.842 & 44,2 & 16,7 \\
\hline & AHS, BHS & 1.750 & 46,3 & 16,1 \\
\hline & $\begin{array}{l}\text { Kolleg, Uni-LG, } \\
\text { hochschulverwandte } \\
\text { Lehranstalten }\end{array}$ & 409 & 57,2 & 4,4 \\
\hline & $\begin{array}{l}\text { Universität, } \\
\text { Fachhochschule }\end{array}$ & 1.025 & 49,7 & 9,0 \\
\hline \multirow{2}{*}{ Erwerbstätig } & Nicht erwerbstätig & 4.169 & 35,5 & 30,7 \\
\hline & Erwerbstätig & 7.488 & 44,5 & 69,3 \\
\hline \multirow{2}{*}{$\begin{array}{l}\text { Beruflicher Status } \\
\text { (Leitungs-funktion) }\end{array}$} & $\begin{array}{l}\text { Keine leitende } \\
\text { Funktion }\end{array}$ & 9.438 & 39,0 & 76,5 \\
\hline & Leitende Funktion & 2.219 & 51,0 & 23,5 \\
\hline \multirow{2}{*}{$\begin{array}{l}\text { Wohnrechts- } \\
\text { verhältnis }\end{array}$} & $\begin{array}{l}\text { Miete, Untermiete, } \\
\text { sonstiges }\end{array}$ & 3.516 & 31,5 & 23,0 \\
\hline & $\begin{array}{l}\text { Wohnungs- bzw. } \\
\text { Hauseigentum }\end{array}$ & 8.141 & 45,5 & 77,0 \\
\hline \multirow{2}{*}{ Familienstand } & Nicht verheiratet & 4.836 & 36,8 & 37,0 \\
\hline & Verheiratet & 6.821 & 44,5 & 63,0 \\
\hline \multirow{3}{*}{ Urbanisierungs-grad } & $\begin{array}{l}\text { niedrige } \\
\text { Bevölkerungsdichte }\end{array}$ & 5.183 & 46,9 & 50,5 \\
\hline & $\begin{array}{l}\text { mittlere } \\
\text { Bevölkerungsdichte }\end{array}$ & 3.106 & 43,0 & 27,8 \\
\hline & $\begin{array}{l}\text { hohe } \\
\text { Bevölkerungsdichte }\end{array}$ & 3.368 & 31,1 & 21,8 \\
\hline Gesamt & & 11.657 & 41,3 & 100 \\
\hline
\end{tabular}

Quelle: Mikrozensus-Zusatzerhebung 2006/Q4; eigene Berechnung. 
Tabelle 37: Beteiligungsquoten - Formelle Freiwilligenarbeit und Leitungsfunktion in der Freiwilligenarbeit nach dem Lebensunterhalt

\begin{tabular}{|c|c|c|c|c|}
\hline & \multicolumn{2}{|c|}{$\begin{array}{l}\text { Beteiligungsquote } \\
\text { formelle } \\
\text { Freiwilligenarbeit }\end{array}$} & \multicolumn{2}{|c|}{$\begin{array}{c}\text { Leitungsquote } \\
\text { in der Freiwilligenarbeit }\end{array}$} \\
\hline & $\mathbf{N}$ & $\%$ & $\mathbf{N}$ & $\%$ \\
\hline In Elternkarenz & 245 & 21,6 & 53 & 20,8 \\
\hline Arbeitslos & 298 & 28,9 & 86 & 18,6 \\
\hline Dauerhaft Arbeitsunfähig & 84 & 31,0 & 26 & 26,9 \\
\hline Pensionist(in) & 2.423 & 33,3 & 808 & 27,0 \\
\hline Ausschließlich haushaltsführend & 914 & 39,6 & 362 & 16,3 \\
\hline Anderes & 67 & 43,3 & 29 & 34,5 \\
\hline Erwerbstätig (auch Lehrling) & 6.880 & 44,9 & 3.087 & 29,9 \\
\hline Schüler(in)/Student(in) & 697 & 48,1 & 335 & 11,6 \\
\hline Präsenz-/Zivildiener & 49 & 55,1 & 26 & 23,1 \\
\hline Gesamt & & 41,3 & & 26,8 \\
\hline $\mathrm{N}$ & 11.657 & 4.814 & 4.812 & 1.288 \\
\hline
\end{tabular}

Quelle: Mikrozensus-Zusatzerhebung 2006/Q4; eigene Berechnung. 


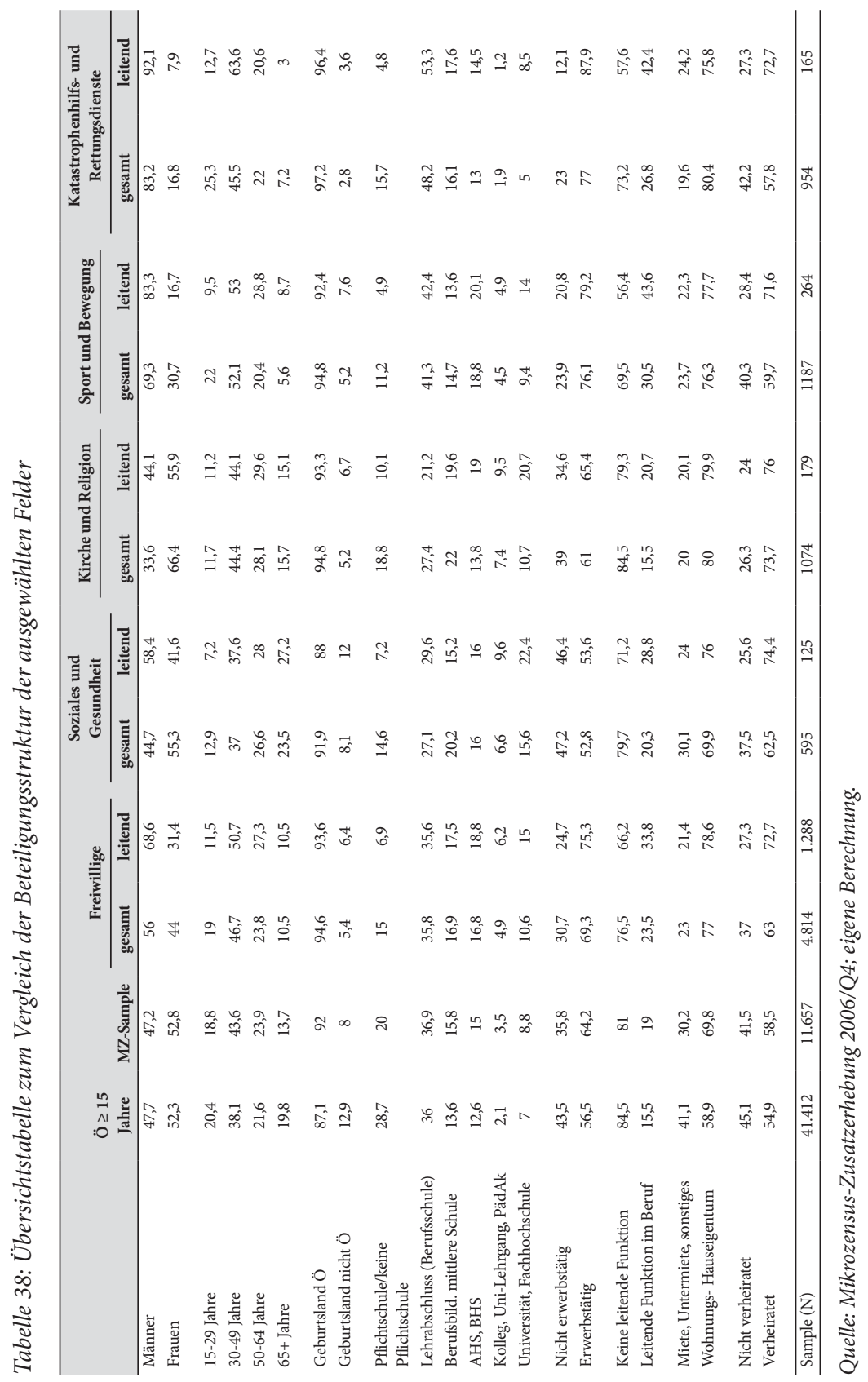




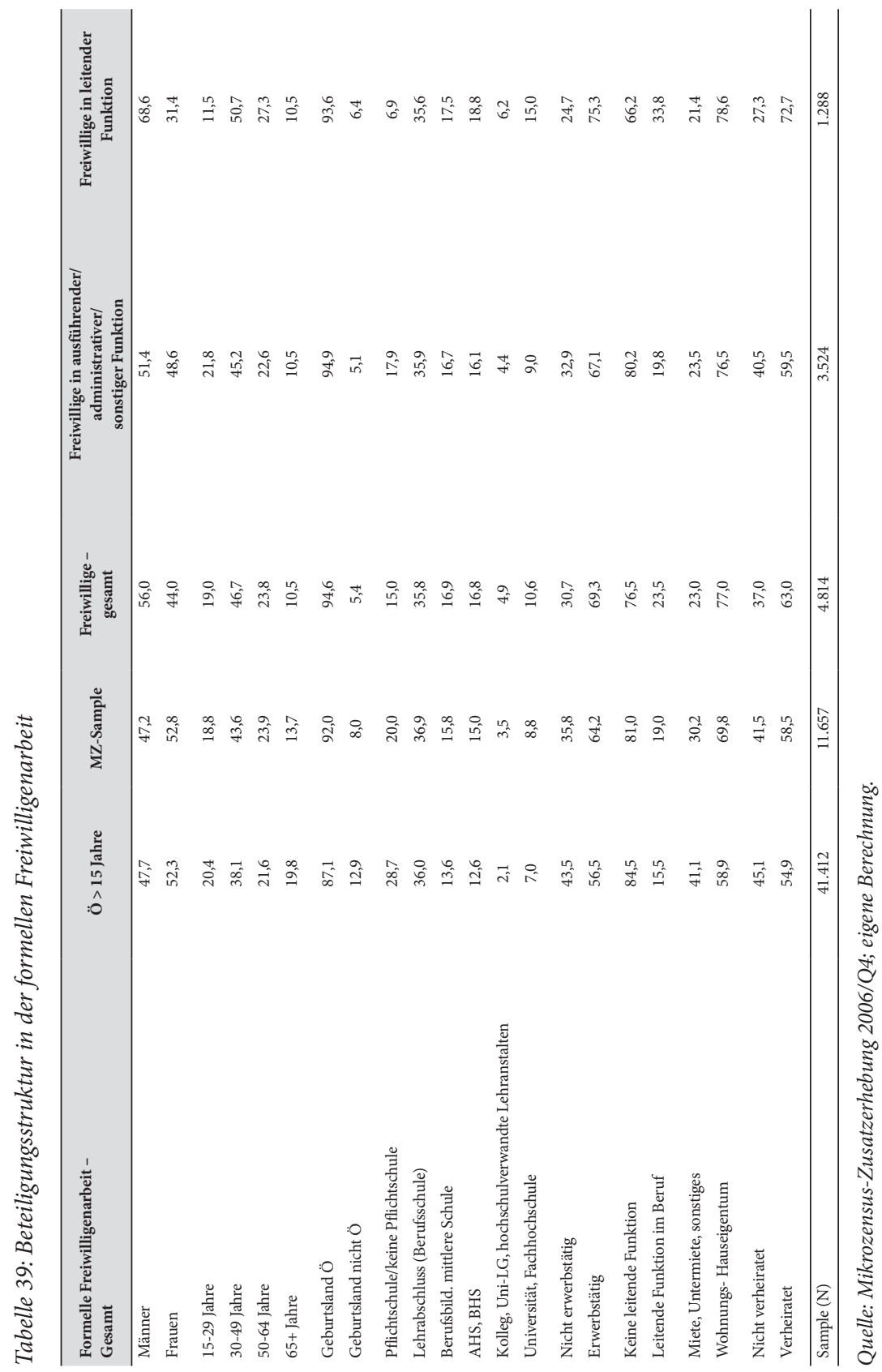




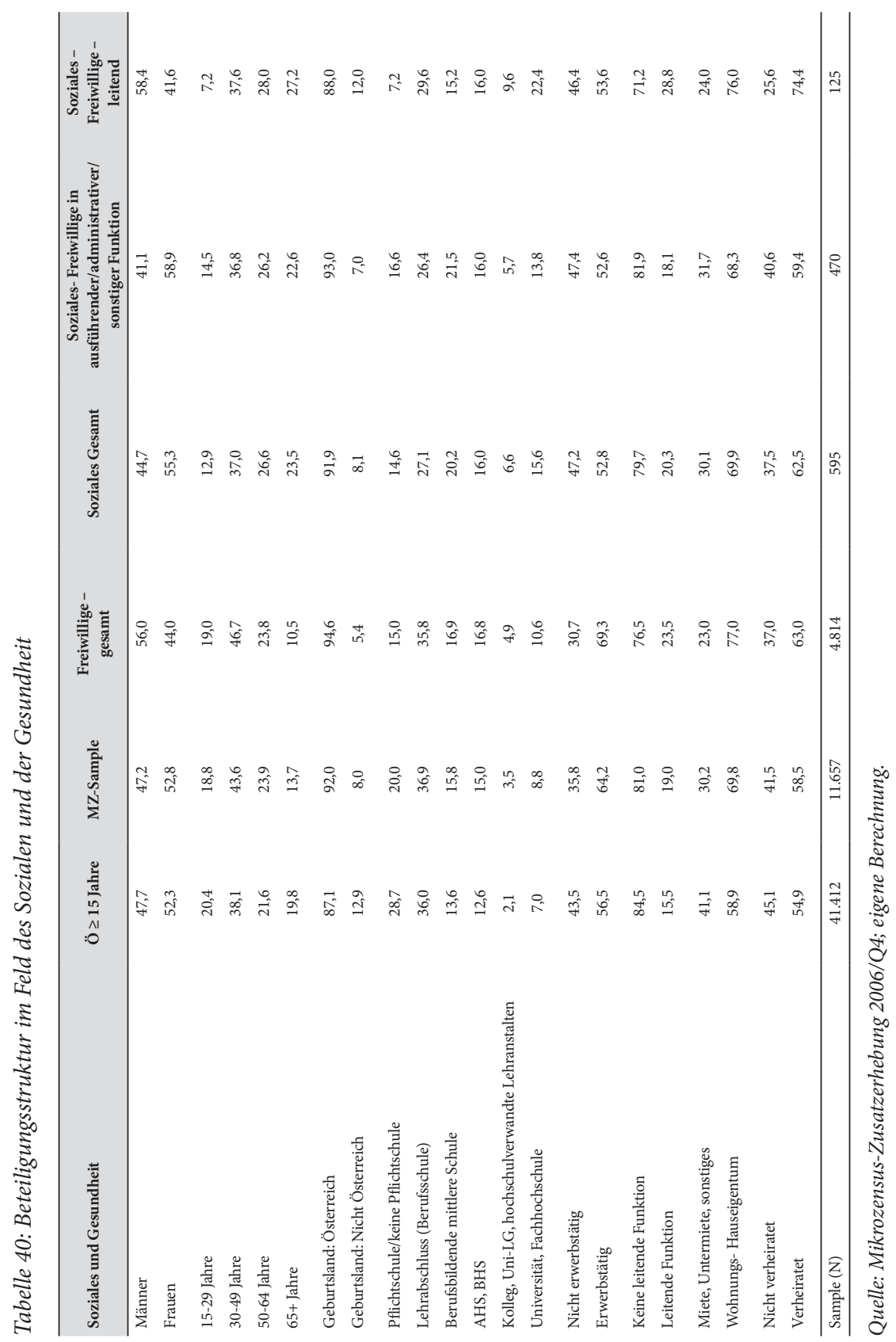




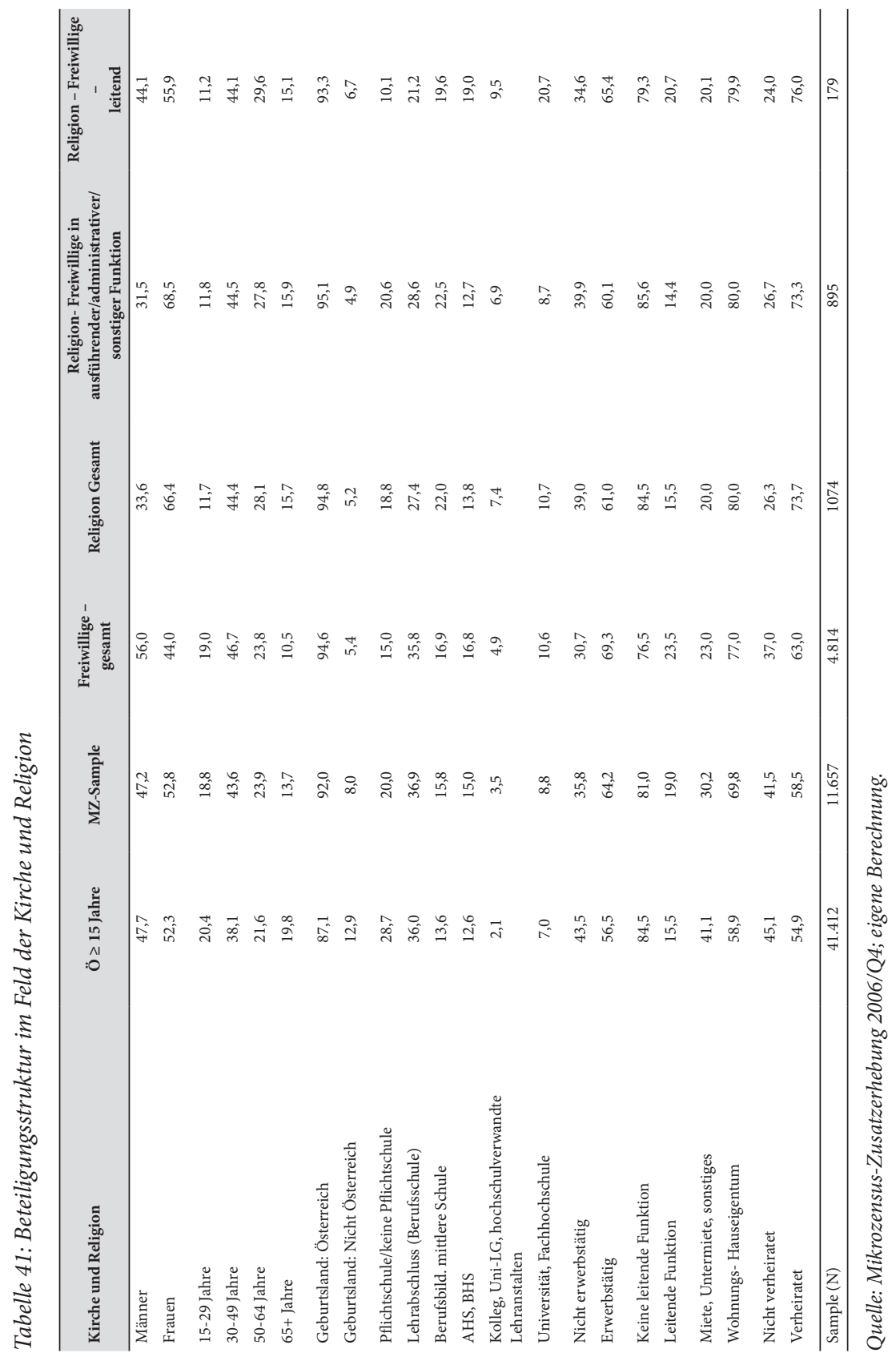




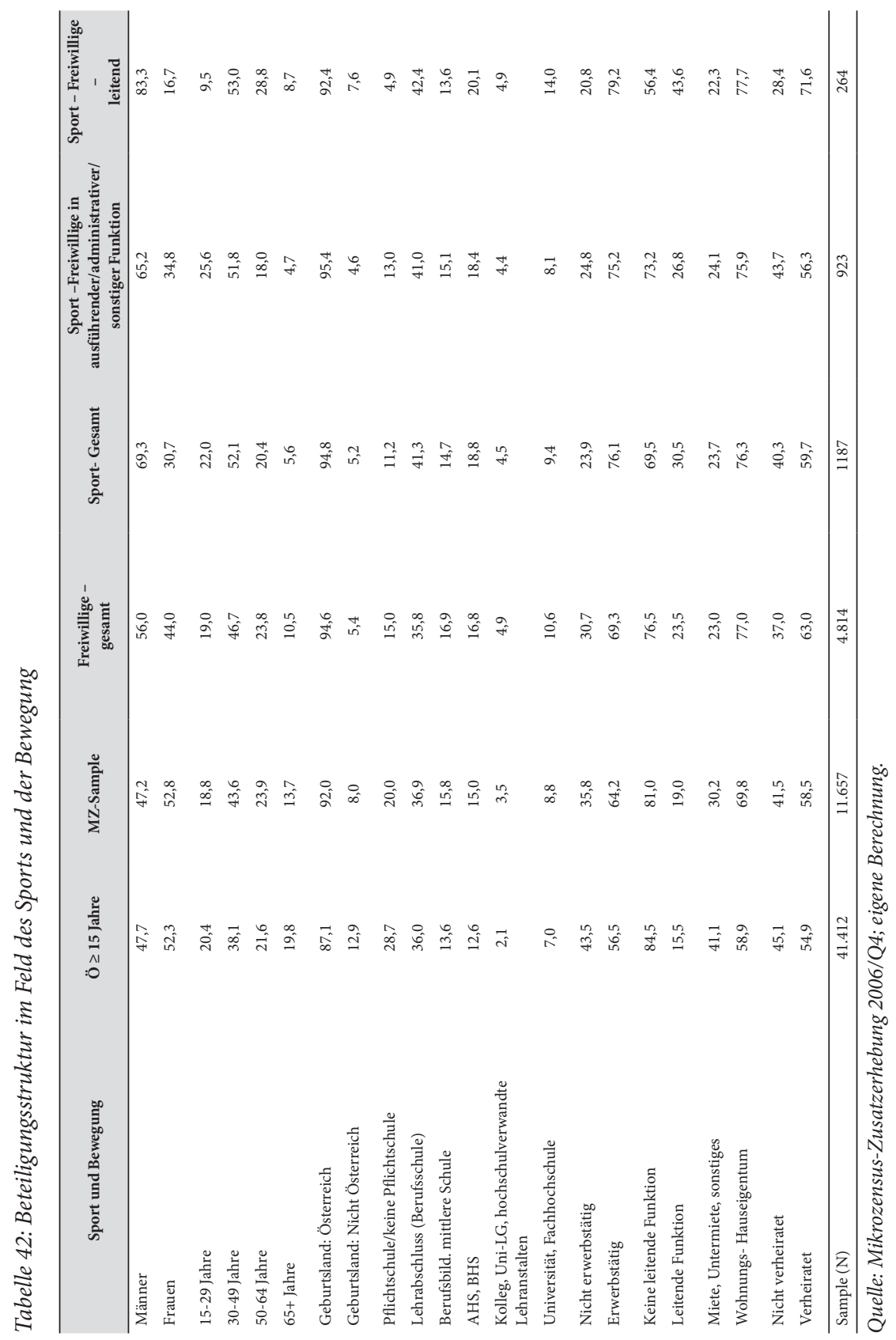




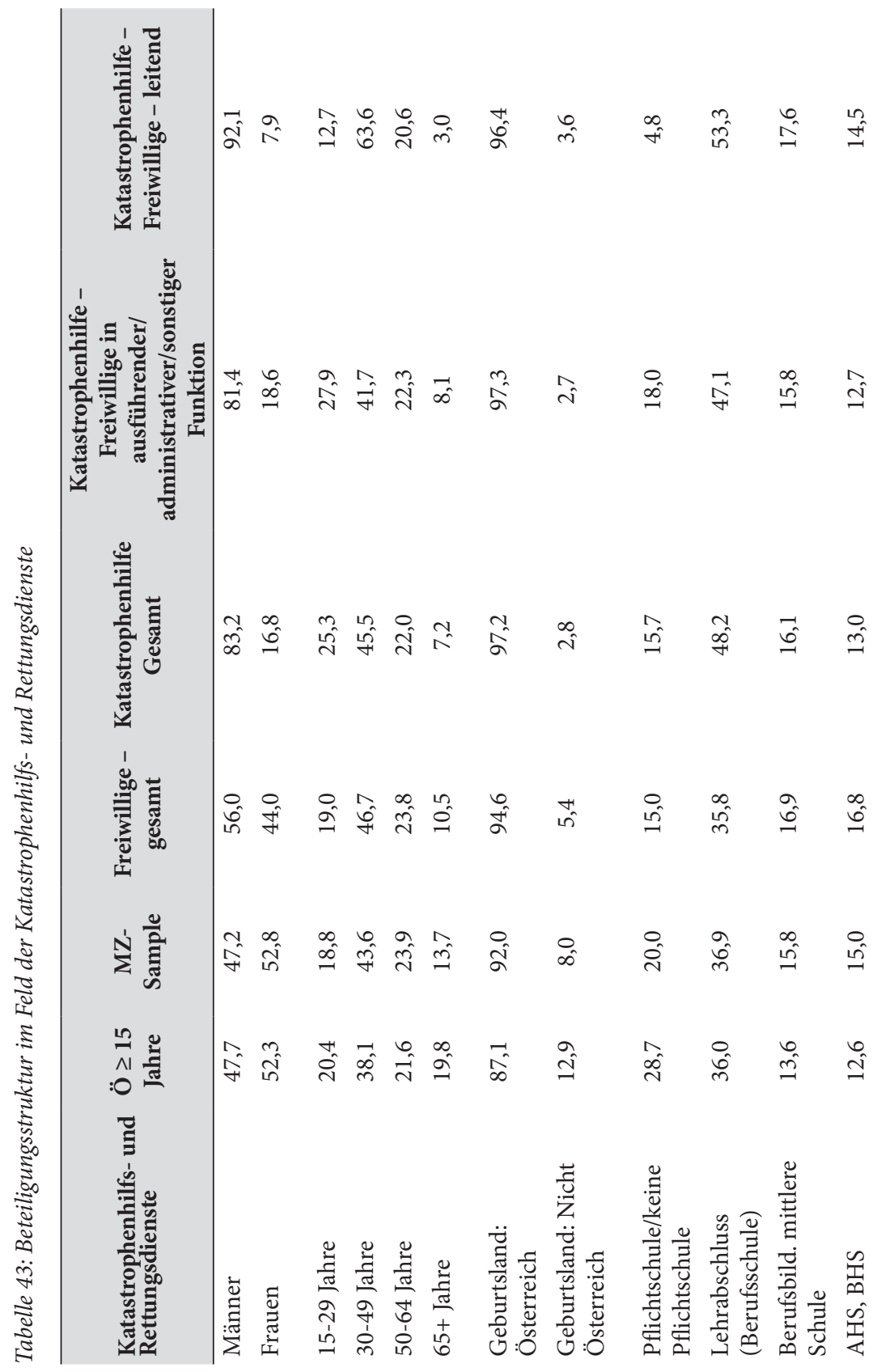




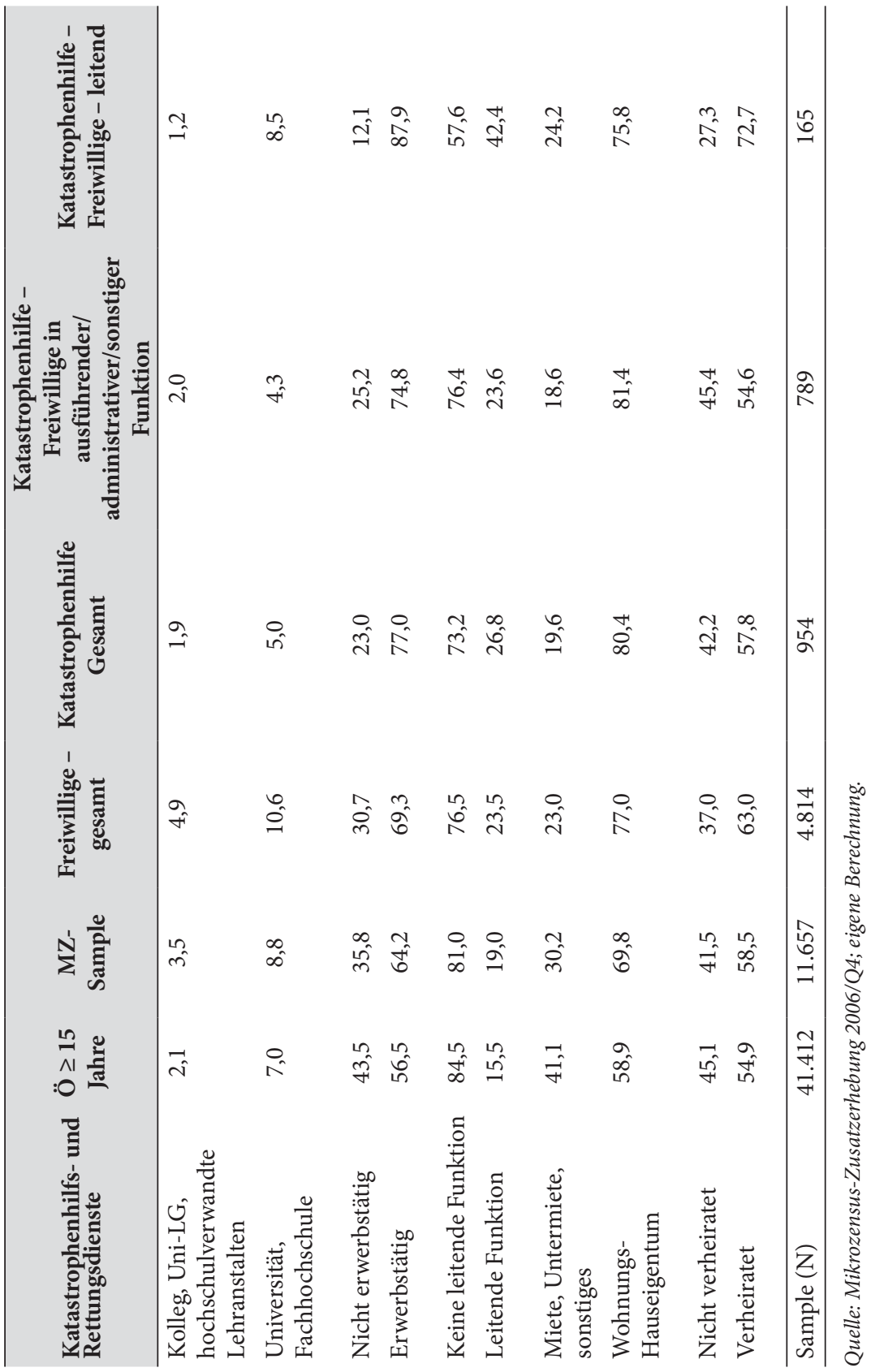




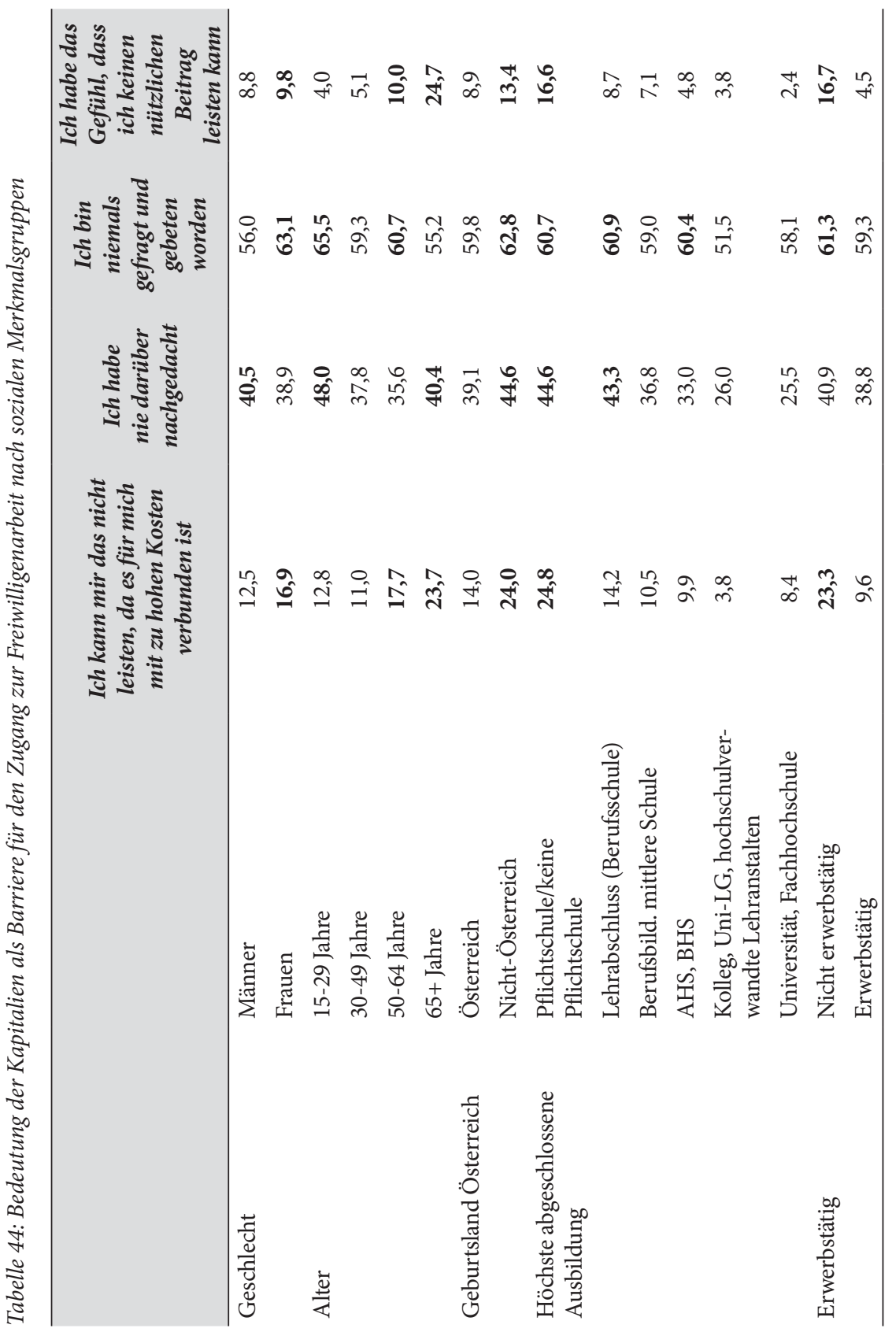




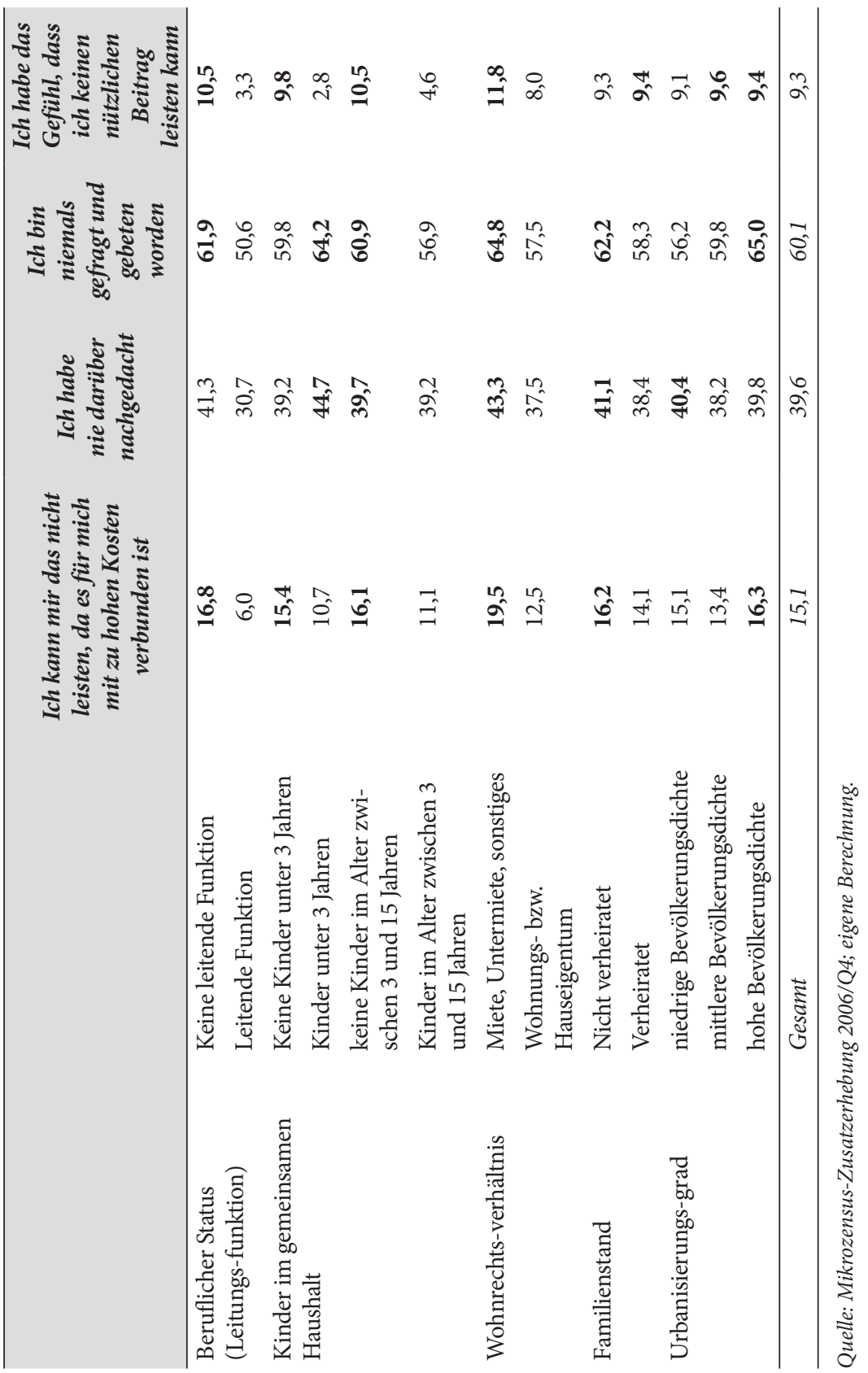


Tabelle 45: Logistische Regression - Determinanten des Zugangs zur Freiwilligenarbeit (inkl. Erwerbsarbeit und Leitungsfunktion)

\begin{tabular}{|c|c|c|c|c|c|c|c|c|c|}
\hline & \multicolumn{9}{|c|}{ Abhängige Variable: Formelle Freiwilligenarbeit } \\
\hline & \multicolumn{3}{|c|}{ Model I } & \multicolumn{3}{|c|}{ Model II } & \multicolumn{3}{|c|}{ Model III } \\
\hline & Beta & SE & $\operatorname{Exp}(B)$ & Beta & SE & $\operatorname{Exp}(B)$ & Beta & SE & $\operatorname{Exp}(B)$ \\
\hline Frauen (Ref. Männer) & $-0,586$ & 0,039 &, $557^{* * *}$ & $-0,581$ & 0,039 &, $559^{* * *}$ & $-0,562$ & 0,04 & $0,570^{* * *}$ \\
\hline Alter & $-0,011$ & 0,001 &, $990^{* * *}$ & $-0,01$ & 0,001 &, $990^{* * *}$ & $-0,01$ & 0,001 & $0,990^{* * *}$ \\
\hline $\begin{array}{l}\text { Geburtsland (Ref. } \\
\text { Österreich) }\end{array}$ & $-0,555$ & 0,08 &, $\mathbf{5 7 4}^{* * *}$ & $-0,551$ & 0,08 &, $576^{* * *}$ & $-0,543$ & 0,08 & $0,581^{* * *}$ \\
\hline Bildungsgrad & 0,203 & 0,014 & $1,225^{* * *}$ & 0,2 & 0,014 & $1,222^{* * *}$ & 0,196 & 0,014 & $1,217^{* * *}$ \\
\hline $\begin{array}{l}\text { Leitenden Funktion } \\
\text { im Beruf }\end{array}$ & & & & & & & 0,135 & 0,054 & $1,145^{* *}$ \\
\hline $\begin{array}{l}\text { Erwerbtätigkeit ((Ref. } \\
\text { Erwerbslos, Arbeitslos, } \\
\text { in Pension, Präsenz- } \\
\text { oder Zivildiener }))\end{array}$ & & & & 0,049 & 0,046 & 1,050 & 0,01 & 0,049 & $1,010^{\text {n.s. }}$ \\
\hline $\begin{array}{l}\text { Wohnrechtsverhältnis } \\
\text { Eigentum (Ref. Miete, } \\
\text { Untermiete, sonstiges) }\end{array}$ & 0,352 & 0,047 & $1,422^{* * *}$ & 0,352 & 0,047 & $1,422^{* * *}$ & 0,351 & 0,047 & $1,421^{* * *}$ \\
\hline $\begin{array}{l}\text { Verheiratet (Ref. } \\
\text { Ledig, Geschieden, } \\
\text { Verwitwet) }\end{array}$ & 0,342 & 0,044 & $1,407^{* * *}$ & 0,334 & 0,045 & $1,396^{* * *}$ & 0,33 & 0,045 & $1,390^{* * *}$ \\
\hline Bevölkerungsdichte & $-0,302$ & 0,025 &, $739^{* * *}$ & $-0,301$ & 0,025 &, $740^{* * *}$ & $-0,301$ & 0,026 & $0,740^{* * *}$ \\
\hline Konstante & 1,141 & 0,133 & $3,130^{* * *}$ & 1,081 & 0,145 & $2,947^{* * *}$ & 1,068 & 0,145 & $2,911^{* * *}$ \\
\hline $\mathrm{N}$ & & 11.657 & & & 11.657 & & & 11.657 & \\
\hline $\mathrm{Chi}^{2} / \mathrm{df}$ & & $930 / 7$ & & & $931 / 8$ & & & $936 / 9$ & \\
\hline -2 Log-Likelihood & & 4875,5 & & & 4874,3 & 385 & & $14.868,1$ & 17 \\
\hline $\begin{array}{l}\text { Pseudo R }{ }^{2} \\
\text { (Nagelkerke’s) }\end{array}$ & & 0,103 & & & 0,103 & & & 0,104 & \\
\hline
\end{tabular}

Quelle: Mikrozensus-Zusatzerhebung 2006/Q4; eigene Berechnung; $\left(p^{*}<0.05 ; p^{* *}<0.01 ; p^{* * *}<0.001\right)$. 
Tabelle 46: Logistische Regression - Determinanten des Zugangs zu leitenden Positionen in der Freiwilligenarbeit

\begin{tabular}{|c|c|c|c|c|c|c|c|c|c|}
\hline & \multicolumn{9}{|c|}{ Abhängige Variable: Leitende Funktion in der Freiwilligenarbeit } \\
\hline & \multicolumn{3}{|c|}{ Model I } & \multicolumn{3}{|c|}{ Model II } & \multicolumn{3}{|c|}{ Model III } \\
\hline & Beta & SE & $\operatorname{Exp}(B)$ & Beta & SE & $\operatorname{Exp}(B)$ & Beta & SE & $\operatorname{Exp}(B)$ \\
\hline $\begin{array}{l}\text { Erwerbsarbeit - lei- } \\
\text { tende Funktion (ref. } \\
\text { keine) }\end{array}$ & & & & & & & 0,348 & 0,083 & $1,416^{* * *}$ \\
\hline $\begin{array}{l}\text { Erwerbstätig (ref. } \\
\text { nein) }\end{array}$ & & & & 0,342 & 0,086 & $1,407^{* * *}$ & 0,213 & 0,092 & $1,238^{\star}$ \\
\hline Frauen (Ref. Männer) & $-0,770$ & 0,070 & $0,463^{* * *}$ & $-0,736$ & 0,071 & $0,479^{* * *}$ & $-0,670$ & 0,073 & $0,512^{* * *}$ \\
\hline Alter & 0,007 & 0,003 & $1,007^{* *}$ & 0,012 & 0,003 & $1,012^{* * *}$ & 0,011 & 0,003 & $1,011^{* * *}$ \\
\hline $\begin{array}{l}\text { Geburtsland (Ref. } \\
\text { Österreich) }\end{array}$ & 0,147 & 0,146 & 1,158 & 0,184 & 0,146 & 1,202 & 0,200 & 0,146 & 1,221 \\
\hline Bildungsgrad & 0,221 & 0,023 & $1,247^{* * *}$ & 0,205 & 0,023 & $1,227^{* * *}$ & 0,192 & 0,023 & $1,212^{* * *}$ \\
\hline $\begin{array}{l}\text { Wohnrechtsverhältnis } \\
\text { Eigentum (Ref. Miete, } \\
\text { Untermiete, sonstiges) }\end{array}$ & 0,018 & 0,085 & 1,018 & 0,019 & 0,085 & 1,019 & 0,014 & 0,085 & 1,014 \\
\hline $\begin{array}{l}\text { Verheiratet (Ref. } \\
\text { Ledig, Geschieden, } \\
\text { Verwitwet) }\end{array}$ & 0,513 & 0,081 & $1,671^{* * *}$ & 0,443 & 0,082 & $1,557^{* * *}$ & 0,423 & 0,083 & $1,527^{* * *}$ \\
\hline Bevölkerungsdichte & $-0,072$ & 0,045 & 0,931 & $-0,069$ & 0,045 & 0,933 & $-0,072$ & 20,045 & 0,931 \\
\hline Konstante & $-1,304$ & 0,230 & $0,271^{* * *}$ & $-1,758$ & 0,259 & $0,172^{* * *}$ & $-1,779$ & 0,259 & $0,169^{* * *}$ \\
\hline $\mathrm{N}$ & & 4.812 & & & 4.812 & & & 4.812 & \\
\hline $\mathrm{Chi}^{2} / \mathrm{df}$ & & $299 / 7$ & & & $315 / 8$ & & & $333 / 9$ & \\
\hline -2 Log-Likelihood & & 5291,68 & & & 5275,5 & & & 5258,3 & 39 \\
\hline $\begin{array}{l}\text { Pseudo R }{ }^{2} \\
\text { (Nagelkerke's) }\end{array}$ & & 0,088 & & & 0,092 & & & 0,097 & \\
\hline
\end{tabular}

Quelle: Mikrozensus-Zusatzerhebung 2006/Q4; eigene Berechnung; $\left(p^{*}<0.05 ; p^{* *}<0.01 ; p^{* * *}<0.001\right)$. 
Tabelle 47: Logistische Regression - Determinanten des Zugangs zur Erwerbsarbeit (inkl. Freiwilligenarbeit)

\begin{tabular}{|c|c|c|c|c|c|c|}
\hline & \multicolumn{6}{|c|}{ Abhängige Variable: Erwerbsarbeit } \\
\hline & \multicolumn{3}{|c|}{ Model I } & \multicolumn{3}{|c|}{ Model II } \\
\hline & Beta & SE & $\overline{\operatorname{Exp}(B)}$ & Beta & SE & $\operatorname{Exp}(B)$ \\
\hline Freiwilligenarbeit (ref. nein) & & & & 0,042 & 0,046 & 1,043 \\
\hline Frauen (Ref. Männer) & $-0,518$ & 0,044 & $0,596^{* * *}$ & $-0,512$ & 0,045 & $0,599^{* * *}$ \\
\hline Alter & $-0,064$ & 0,002 & $0,938^{* * *}$ & $-0,064$ & 0,002 & $0,938^{* * *}$ \\
\hline Geburtsland (Ref. Österreich) & $-0,412$ & 0,082 & $0,663^{* * *}$ & $-0,407$ & 0,082 & $0,666^{* * *}$ \\
\hline Bildungsgrad & 0,313 & 0,017 & $1,368^{* * *}$ & 0,311 & 0,017 & $1,365^{* * *}$ \\
\hline $\begin{array}{l}\text { Wohnrechtsverhältnis } \\
\text { Eigentum (Ref. Miete, } \\
\text { Untermiete, sonstiges) }\end{array}$ & $-0,070$ & 0,052 & 0,932 & $-0,073$ & 0,052 & 0,930 \\
\hline $\begin{array}{l}\text { Verheiratet (Ref. Ledig, } \\
\text { Geschieden, Verwitwet) }\end{array}$ & 0,776 & 0,050 & $2,173^{* * *}$ & 0,773 & 0,051 & $2,166^{* * *}$ \\
\hline Bevölkerungsdichte & $-0,050$ & 0,028 & 0,952 & $-0,047$ & 0,028 & 0,954 \\
\hline Konstante & 3,663 & 0,151 & $38,972^{* * *}$ & 3,633 & 0,155 & $37,815^{* * *}$ \\
\hline $\mathrm{N}$ & & 11.657 & & & 11.657 & \\
\hline $\mathrm{Chi}^{2} / \mathrm{df}$ & & $2740 / 7$ & & & $2741 / 8$ & \\
\hline -2 Log-Likelihood & & 12462,153 & & & 12461,335 & \\
\hline Pseudo R ${ }^{2}$ (Nagelkerke’s) & & 0,287 & & & 0,288 & \\
\hline
\end{tabular}

Quelle: Mikrozensus-Zusatzerhebung 2006/Q4; eigene Berechnung; $\left(p^{*}<0.05 ; p^{* *}<0.01 ; p^{* * *}<0.001\right)$. 
Tabelle 48: Logistische Regression - Determinanten des Zugangs zu leitenden Positionen in der Erwerbsarbeit

\begin{tabular}{|c|c|c|c|c|c|c|c|c|c|}
\hline & \multicolumn{9}{|c|}{ Abhängige Variable: Leitende Funktion in der Erwerbsarbeit } \\
\hline & \multicolumn{3}{|c|}{ Model I } & \multicolumn{3}{|c|}{ Model II } & \multicolumn{3}{|c|}{ Model III } \\
\hline & Beta & SE & $\overline{\operatorname{Exp}(B)}$ & Beta & SE & $\operatorname{Exp}(B)$ & Beta & SE & $\operatorname{Exp}(B)$ \\
\hline $\begin{array}{l}\text { Freiwilligenarbeit } \\
\text { leitende Funktion (ref. } \\
\text { keine) }\end{array}$ & & & & & & & 0,379 & 0,093 & $1,461^{* * *}$ \\
\hline $\begin{array}{l}\text { Freiwilligenarbeit (ref. } \\
\text { nein) }\end{array}$ & & & & 0,102 & 0,061 & 1,108 & $-0,007$ & 0,067 & 0,993 \\
\hline Frauen (Ref. Männer) & $-1,165$ & 0,061 & $0,312^{* * *}$ & $-1,152$ & 0,061 & $0,316^{* *+}$ & $-1,132$ & 0,062 &, $322^{* * *}$ \\
\hline Alter & 0,031 & 0,003 & $1,032^{* * *}$ & 0,031 & 0,003 & $1,032^{* * *}$ & 0,031 & 0,003 & $1,031^{* * *}$ \\
\hline $\begin{array}{l}\text { Geburtsland (Ref. } \\
\text { Österreich) }\end{array}$ & $-0,626$ & 0,126 & $0,535^{* * *}$ & $-0,612$ & 0,126 & $0,542^{* * *}$ & $-0,613$ & 0,126 & $0,542^{* * *}$ \\
\hline Bildungsgrad & 0,262 & 0,020 & $1,299^{* * *}$ & 0,257 & 0,020 & $1,293^{* * *}$ & 0,251 & 0,020 & $1,285^{* * *}$ \\
\hline $\begin{array}{l}\text { Wohnrechtsverhältnis } \\
\text { Eigentum (Ref. Miete, } \\
\text { Untermiete, sonstiges) }\end{array}$ & $-0,060$ & 0,070 & 0,942 & $-0,068$ & 0,071 & 0,934 & $-0,063$ & 0,071 & 0,939 \\
\hline $\begin{array}{l}\text { Verheiratet (Ref. } \\
\text { Ledig, Geschieden, } \\
\text { Verwitwet) }\end{array}$ & 0,129 & 0,068 & 1,138 & 0,122 & 0,068 & 1,130 & 0,104 & 0,068 & 1,109 \\
\hline Bevölkerungsdichte & $-0,095$ & 0,038 & $0,910^{*}$ & $-0,086$ & 0,039 & $0,917^{*}$ & $-0,081$ & 0,039 & $0,922^{*}$ \\
\hline Konstante & $-0,496$ & 0,210 & $0,609^{*}$ & $-0,568$ & 0,214 & $0,567^{*}$ & $-0,562$ & 0,214 & $0,570^{*}$ \\
\hline $\mathrm{N}$ & & 6.431 & & & 6.431 & & & 6.430 & \\
\hline $\mathrm{Chi}^{2} / \mathrm{df}$ & & $727 / 7$ & & & $730 / 8$ & & & $747 / 9$ & \\
\hline -2 Log-Likelihood & & 6941,51 & & & 6938,65 & & & 6921,2 & 25 \\
\hline $\begin{array}{l}\text { Pseudo R }{ }^{2} \\
\text { (Nagelkerke's) }\end{array}$ & & 0,153 & & & 0,154 & & & 0,157 & \\
\hline
\end{tabular}

Quelle: Mikrozensus-Zusatzerhebung 2006/Q4; eigene Berechnung; $\left(p^{*}<0.05 ; p^{* *}<0.01 ; p^{* * *}<0.001\right)$. 


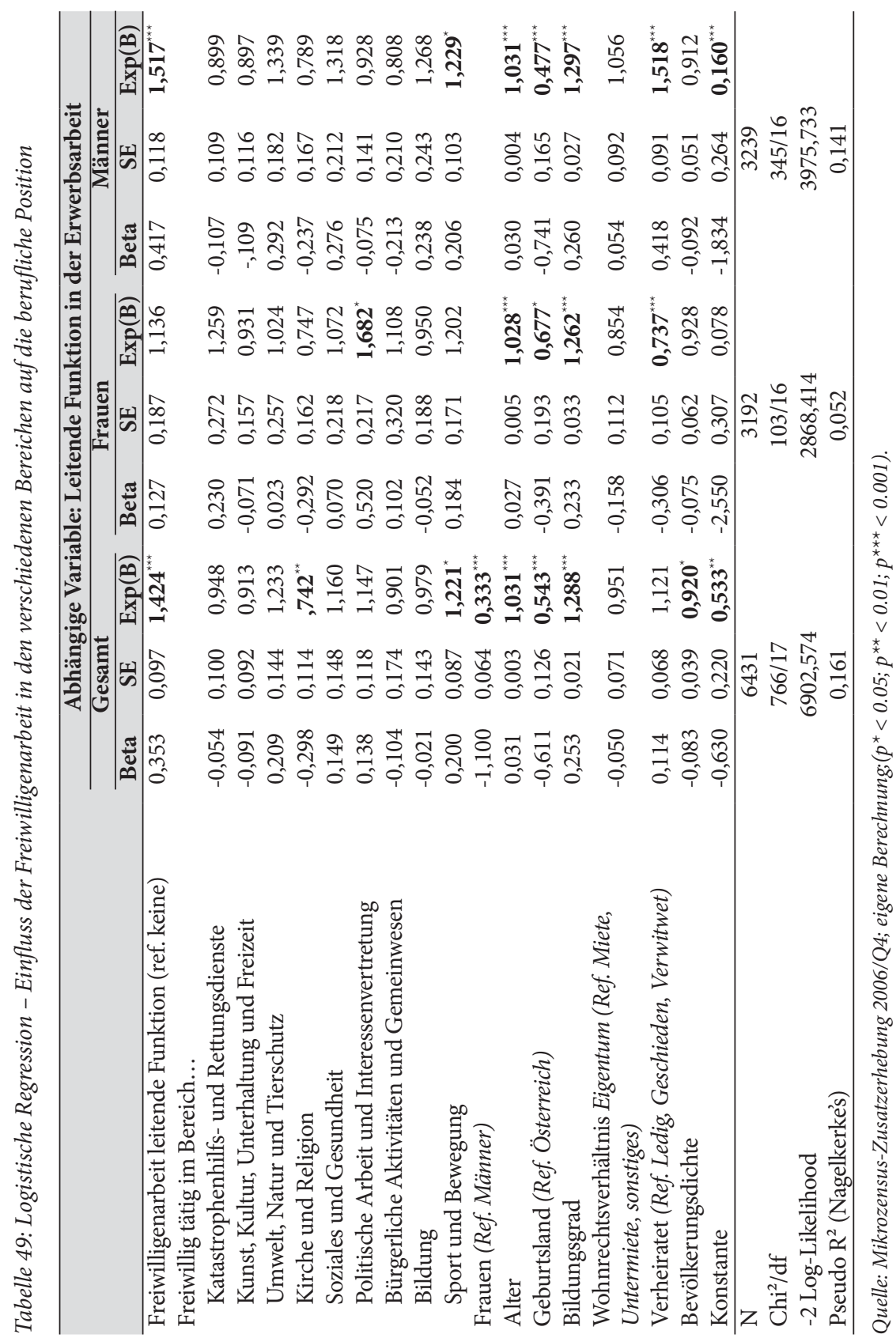




\section{Anhang 3: Fragebogen der empirischen Erhebung \\ Fragebogen - Mikrozensus-Zusatzerhebung (2006) \\ (NPO-INSTITUT 2006)}

\section{Fragebogengliederung:}

Einführungstext

Teil A: für alle Befragten (Feststellung ehrenamtlicher Tätigkeiten in den 10 Bereichen:)

Fragen zur Erfassung von formellen ehrenamtlichen Tätigkeiten:

a. Fragen zum Bereich der Katastrophenhilfs- und Rettungsdienste

b. Fragen zum Bereich Kunst, Kultur, Unterhaltung und Freizeit

c. Fragen zum Bereich Umwelt, Natur und Tierschutz

d. Fragen zum kirchlichen oder religiösen Bereich

e. Fragen zum Sozial- und Gesundheitsbereich

f. Fragen zum Bereich politische Arbeit und Interessenvertretung

g. Fragen zum Bereich bürgerliche Aktivitäten und Gemeinwesen

h. Fragen zum Bereich Bildung

i. Fragen zum Bereich Sport und Bewegung

Fragen zur Erfassung von informellen ehrenamtlichen Tätigkeiten

j. Fragen zum Bereich der Nachbarschaftshilfe

Teil B1: für alle ehrenamtlich Tätigen (Motive für ehrenamtliches Engagement)

Teil B2: für alle nicht ehrenamtlich Tätigen (Gründe für kein ehrenamtliches Engagement)

\section{Einführungstext:}

Im Rahmen des Mikrozensus „neu“ wurde vom Bundesministerium für soziale Sicherheit, Generationen und Konsumentenschutz eine weitere sehr wichtige Studie in Auftrag gegeben. Es geht darum festzustellen, was BürgerInnen in verschiedenen Bereichen für die Gesellschaft unbezahlt und freiwillig leisten.

Wir stellen Ihnen dazu nun einige Fragen. Bitte beantworten Sie diese nach bestem Wissen und Gewissen. 


\section{Teil A: Für alle Befragten (Feststellung ehrenamtlicher Tätigkeiten in den 10 Bereichen)}

Es gibt viele Möglichkeiten, sich ehrenamtlich für eine Organisation/einen Verein zu betätigen.

Bitte beachten Sie bei der Beantwortung der Fragen, dass eine Tätigkeit dann als „ehrenamtlich“ bezeichnet wird, wenn diese:

1. freiwillig ausgeübt wird. d. h. ohne gesetzliche Verpflichtung (Zivildienst sowie Berufspraktikum gelten somit nicht als ehrenamtlich)

2. unbezahlt ist (Bei Nachfrage der Apn bitte darauf hinweisen, dass eine Tätigkeit auch dann ehrenamtlich sein kann, wenn für diese $z$. B. Sachkosten ersetzt werden.)

Ich lese Ihnen nun 10 Bereiche vor, in denen man ehrenamtlich tätig sein kann.

Fragen zur Erfassung von formellen ehrenamtlichen Tätigkeiten:

\section{a Fragen zum Bereich der Katastrophenhilfs- und Rettungsdienste}

(a1) Sind Sie irgendwo im Bereich Katastrophenhilfs- und Rettungsdienste ehrenamtlich für eine Organisation oder einen Verein tätig? z. B.

... für die die freiwillige Feuerwehr bzw. die Feuerwehrjugend

... für einen Rettungsdienst (z. B. Rotes Kreuz, Samariterbund, Berg-,

Wasser- Höhlenrettung)

... für eine humanitäre Hilfsorganisation bei nationalen oder internationalen Katastrophen oder

Notlagen

... für eine sonstige noch nicht genannte Organisation

1... Ja Apn ist ehrenamtlich tätig

2... Nein Apn ist nicht ehrenamtlich tätig $\rightarrow$ weiter mit b1

(a2) Sind Sie in diesem Bereich (Katastrophenhilfs- und Rettungsdienste) in einer Organisation/einem Verein tätig? Oder sind Sie in zwei/drei/mehr als drei Organisationen/Vereinen tätig?

1... Eine

2... Zwei

3... Drei

4... Mehr als drei 
(a3) Welche Aufgaben erfüllen Sie im Rahmen ihrer Tätigkeiten? (Mehrfachantworten möglich)

1... Kernaufgabe der Organisation (z. B. aktive Einsätze)

2... Administrative/unterstützende Aufgaben (z. B. Büroarbeiten oder Spenden sammeln)

3... Leitende Funktion bzw. FunktionärIn

4... Sonstige Aufgaben

(a4) An wie vielen Tagen haben Sie diese ehrenamtliche(n) Tätigkeit(en) in den vergangenen 12 Monaten (also vergangenes Jahr) insgesamt ausgeführt? Sind das ...

1 ... 1 bis 10 Tage?

2... 11 bis 30 Tage?

3... 31 bis 60 Tage?

4... Mehr als 61 Tage?

5...(nie)

(a5) Handelt es sich dabei um eher regelmäßige Aktivitäten oder zeitlich begrenzte, projektförmige Tätigkeiten?

1... Regelmäßige (z. B. wöchentlich, täglich)

2... Einmalige, zeitlich begrenzte Tätigkeiten (z. B. kurzfristige Hilfe bei Projekt/ Veranstaltung)

3... Sowohl regelmäßig als auch einmalig (keine eindeutige Zuordnung möglich)

(a6) Überlegen Sie bitte, wie viele Stunden Sie für diese Tätigkeit(en) in den letzten 7 Tagen insgesamt aufgewendet haben.

--- Stunden

\section{b Fragen zum Bereich Kunst, Kultur, Unterhaltung und Freizeit}

(b1) Sind Sie irgendwo im Bereich Kunst, Kultur, Unterhaltung und Freizeit ehrenamtlich für eine Organisation oder einen Verein tätig? z. B.

... für eine Musikgruppe, Musikkapelle, Band, einen Chor

... für eine kulturelle Vereinigung (z. B. Faschingsgilde, Trachtengruppe.)

... für eine Theater- oder Tanzgruppe

... für einen Kunstverein, ein Museum

... für einen Geselligkeitsverein (z. B. einen Kollegen- bzw. Kolleginnenverein, Kameradschaftsverein)

... für einen sonstigen noch nicht genannten Verein 
1... Ja Apn ist ehrenamtlich tätig

2... Nein Apn ist nicht ehrenamtlich tätig $\rightarrow$ weiter mit $c 1$

Fragen $b 2$ bis b6 analog $z u$ den Fragen a2 bis a6

\section{c Fragen zum Bereich Umwelt, Natur und Tierschutz}

(c1) Sind Sie irgendwo im Bereich Umwelt, Natur und Tierschutz ehrenamtlich für eine Organisation oder einen Verein tätig? z. B.

... für Natur-, Berg- oder Wandervereine (z. B. Naturfreunde, Alpenverein, Alpenschutzverein)

... für eine Tierschutzorganisation (z. B. Vier Pfoten, WWF...)

... für eine Umweltorganisation (z. B. Greenpeace, Global 2000 ...)

... für einen Obst- und Gartenbauverein

... für einen Tierzuchtverein (z. B. Bienenzucht- und Fischereiverein)

...für einen sonstigen noch nicht genannten Verein

1...Ja Apn ist ehrenamtlich tätig

2...Nein Apn ist nicht ehrenamtlich tätig $\rightarrow$ weiter mit d1

Fragen $c 2$ bis c6 analog $z$ u den Fragen a2 bis a 6

\section{d Fragen zum kirchlichen oder religiösen Bereich}

(d1) Sind Sie im kirchlichen oder religiösen Bereich in einer Organisation/ einem Verein tätig? z. B.

... für ein Beratungs- oder Leitungsgremium einer religiösen Gemeinde (z. B. Pfarrgemeinderat, Presbyterium oder vergleichbare Gremien anderer Religionsgemeinschaften)

... Mitwirkung bei der Gestaltung von religiösen Festen oder Gottesdiensten (z. B. Kirchenchor)

... für eine religiöse Kinder- oder Jugendgruppe (z. B. Jungschar, MinistrantInnen, Erstkommunionsgruppe, Konfirmationsgruppen oder ähnliche Gruppen anderer Religionen)

... Tätigkeiten mit Seniorinnen und Senioren

... für religiöse Organisationen (z. B. die katholische Frauen- oder Männerbewegung oder ähnliche Organisationen anderer Religionen)

1... Ja Apn ist ehrenamtlich tätig

2... Nein Apn ist nicht ehrenamtlich tätig $\rightarrow$ weiter mit e1 
Fragen $d 2$ bis $d 6$ analog $z u$ den Fragen a 2 bis a 6

\section{e Fragen zum Sozial- und Gesundheitsbereich}

(e1) Sind Sie im Sozial- und Gesundheitsbereich ehrenamtlich für eine Organisation oder einen Verein tätig?

z. B.

... für eine Sozial- bzw. Hilfsorganisation (in der Betreuung von Kindern, Jugendlichen, Familien, Menschen mit Behinderung, älteren oder pflegebedürftigen Menschen oder anderen Menschen mit besonderem Hilfs- und Betreuungsbedarf)

... für einen Familien-, Omadienst

... für ein Jugendzentren oder eine Jugendgruppe (wie z. B. die PfadfinderInnen)

... für eine Seniorenorganisation, Pensionistenverband, Seniorenbund o. a.

... für eine Selbsthilfegruppe (von Eltern, Frauen oder zu bestimmten Krankheiten ...)

... für eine Einrichtung der Arbeitslosenhilfe oder eine Selbstorganisation von Migrantinnen und Migranten

... für eine Organisation zur Betreuung von Straffälligen oder Verbrechensopfern, Bewährungshilfe

... für einen Besuchs- oder Begleitdienst (z. B. Pensionisten- oder Pflegeheim, Psychiatrie, Krankenhaus)

... für einen sonstigen noch nicht genannten Verein

1... Ja Apn ist ehrenamtlich tätig

2... Nein Apn ist nicht ehrenamtlich tätig $\rightarrow$ weiter mit $f 1$

Fragen e2 bis e6 analog zu den Fragen a2 bis a6

\section{f Fragen zum Bereich politische Arbeit und Interessenvertretung}

(f1) Sind Sie irgendwo im Bereich politische Arbeit und Interessenvertretung ehrenamtlich für eine Organisation oder einen Verein tätig? z. B.

... für den Betriebsrat, die Gewerkschaft, den Berufsverband, Wirtschaftsverband oder Bauernbund etc.

... für eine politische Initiative oder Partei

... für den Gemeinde- oder Stadtrat

... für ein Menschenrechts- oder Solidaritätsprojekt

... für eine Organisation im Bereich der Entwicklungshilfe (z. B. Südwind)

... für einen sonstigen noch nicht genannten Verein 
1... Ja Apn ist ehrenamtlich tätig

2... Nein Apn ist nicht ehrenamtlich tätig $\rightarrow$ weiter mit $g 1$

Fragen $f 2$ bis $f 6$ analog $z u$ den Fragen a 2 bis a 6

\section{g Fragen zum Bereich bürgerliche Aktivitäten und Gemeinwesen}

(g1) Sind Sie irgendwo im Bereich bürgerliche Aktivitäten und Gemeinwesen an Ihrem Wohnort ehrenamtlich für eine Organisation oder einen Verein tätig? z. B.

... für einen Fremdenverkehrs- bzw. Tourismusverein

... für einen Ortsentwicklungs- (z. B. Siedlungs-, Verschönerungsverein) oder Verkehrsentwicklungsverein

... für eine BürgerInneninitiative (für Wohnraumgestaltung, nachhaltige Entwicklung o. Ä.)

... für ein BürgerInnenbüro für Jung und Alt oder ein Generationennetzwerk o. Ä.

... für Arbeiten im nahen Wohnumfeld (Nachbarschaftszentrum, Spielplatzbau, o. Ä.)

... für einen sonstigen noch nicht genannten Verein

1... Ja Apn ist ehrenamtlich tätig

2... Nein Apn ist nicht ehrenamtlich tätig $\rightarrow$ weiter mit h1

Fragen g2 bis g6 analog zu den Fragen a2 bis a6

\section{h Fragen zum Bereich Bildung}

(h1) Sind Sie irgendwo im Bereich Bildung ehrenamtlich für eine Organisation oder einen Verein tätig? z. B.

... für einen Elternverein oder eine SchülerInnenvertretung

... für eine Weiterbildungseinrichtung, die Erwachsenenbildung (z. B. Bildungswerk)

... für ein Lernhilfezentrum oder eine Bibliothek

... für eine sonstige noch nicht genannten Organisation

1... Ja Apn ist ehrenamtlich tätig

2... Nein Apn ist nicht ehrenamtlich tätig $\rightarrow$ weiter mit i1

Fragen $h 2$ bis h6 analog zu den Fragen a2 bis a6 


\section{i Fragen zum Bereich Sport und Bewegung}

(i1) Sind Sie irgendwo im Bereich Sport und Bewegung ehrenamtlich für eine Organisation oder einen Verein tätig? z. B.

... für einen Sport- oder Turnverein (z. B. Fußball-, Tennisverein)

... für einen Fachverband oder eine sonstige Sportorganisation

... für eine Bewegungsgruppe (z. B. SeniorInnengymnastik)

... für einen sonstigen noch nicht genannten Verein

\section{1... Ja Apn ist ehrenamtlich tätig}

2... Nein Apn ist nicht ehrenamtlich tätig $\rightarrow$ weiter mit $j 1$

\section{Fragen i2 bis i6 analog zu den Fragen a 2 bis a 6}

\section{Teil B1: für ehrenamtlich bzw. freiwillig Tätige}

(Motive_EA_1) Menschen sind aus ganz unterschiedlichen persönlichen Gründen ehrenamtlich tätig oder leisten unbezahlte, freiwillige Arbeit. Ich lese Ihnen unterschiedliche Beweggründe für ehrenamtliche Arbeit vor. Bitte sagen Sie mir, ob das auf Sie persönlich in folgendem Ausmaß zutrifft: voll und ganz, eher schon, eher nicht oder überhaupt nicht.

\begin{tabular}{|c|c|c|c|c|c|}
\hline & & $\begin{array}{l}\text { voll und } \\
\text { ganz }\end{array}$ & $\begin{array}{l}\text { Eher } \\
\text { schon }\end{array}$ & $\begin{array}{l}\text { eher } \\
\text { nicht }\end{array}$ & $\begin{array}{l}\text { über- } \\
\text { haupt- } \\
\text { nicht }\end{array}$ \\
\hline A & $\begin{array}{l}\text { Ich möchte etwas Nützliches für das } \\
\text { Gemeinwohl beitragen }\end{array}$ & 1 & 2 & 3 & 4 \\
\hline B & Ich möchte damit anderen helfen & 1 & 2 & 3 & 4 \\
\hline $\mathrm{C}$ & Es erweitert meine Lebenserfahrung & 1 & 2 & 3 & 4 \\
\hline $\mathrm{D}$ & Es hilft mir für meinen Beruf & 1 & 2 & 3 & 4 \\
\hline $\mathrm{E}$ & $\begin{array}{l}\text { Ich kann meine Fähigkeiten und Kenntnisse } \\
\text { einbringen }\end{array}$ & 1 & 2 & 3 & 4 \\
\hline $\mathrm{F}$ & Ich treffe Menschen und gewinne Freunde & 1 & 2 & 3 & 4 \\
\hline G & Es bringt mir gesellschaftliche Anerkennung & 1 & 2 & 3 & 4 \\
\hline $\mathrm{H}$ & Es hilft mir, aktiv zu bleiben & 1 & 2 & 3 & 4 \\
\hline I & Es macht mir Spaß & 1 & 2 & 3 & 4 \\
\hline $\mathrm{J}$ & $\begin{array}{l}\text { Es bietet mir die Möglichkeit, meine } \\
\text { Erfahrungen zu teilen }\end{array}$ & 1 & 2 & 3 & 4 \\
\hline K & Es gibt mir die Möglichkeit dazuzulernen & 1 & 2 & 3 & 4 \\
\hline
\end{tabular}




\begin{tabular}{|l|l|lllc|}
\hline $\mathrm{L}$ & $\begin{array}{l}\text { Ich hoffe, dass mir diese Tätigkeit hilft, einen } \\
\text { bezahlten Job zu finden }\end{array}$ & 1 & 2 & 3 & 4 \\
\hline $\mathrm{M}$ & $\begin{array}{l}\text { Ich arbeite ehrenamtlich, weil ich möchte, } \\
\text { dass auch mir geholfen wird, wenn ich Hilfe } \\
\text { benötige. }\end{array}$ & 1 & 2 & 3 & 4 \\
\hline $\mathrm{N}$ & $\begin{array}{l}\text { Ich möchte mich für eine wichtige Sache } \\
\text { engagieren }\end{array}$ & 1 & 2 & 3 & 4 \\
\hline
\end{tabular}

\section{Teil B2: für nicht ehrenamtlich bzw. freiwillig Tätige}

(Motive_NEA_1) Es gibt unterschiedliche Gründe, warum Menschen nicht ehrenamtlich tätig sind oder keine freiwillige, unbezahlte Arbeit leisten. Ich lese Ihnen nun Aussagen vor. Sagen Sie mir bitte, ob diese auf Sie zutreffen oder nicht zutreffen.

\begin{tabular}{|l|l|ll|}
\cline { 3 - 3 } \multicolumn{2}{l|}{} & trifft zu & trifft nicht zu \\
\hline A & Ich bin niemals gefragt oder gebeten worden & 1 & 2 \\
\hline B & Ich habe nie darüber nachgedacht & 1 & 2 \\
\hline C & Ich bin durch familiäre Aufgaben ausgefüllt & 1 & 2 \\
\hline D & $\begin{array}{l}\text { Ich fühle mich durch Krankheit oder Behinderung } \\
\text { nicht in der Lage }\end{array}$ & 1 & 2 \\
\hline E & $\begin{array}{l}\text { Ich kann mir das nicht leisten, da es für mich mit zu } \\
\text { hohen Kosten verbunden ist }\end{array}$ & 1 & 2 \\
\hline F & $\begin{array}{l}\text { Es lässt sich mit meinem Beruf zeitlich nicht } \\
\text { vereinbaren }\end{array}$ & 1 & 2 \\
\hline G & Ich habe schlechte Erfahrungen gemacht & 1 & 2 \\
\hline H & $\begin{array}{l}\text { Ich habe das Gefühl, dass ich keinen nützlichen } \\
\text { Beitrag leisten kann }\end{array}$ & 1 & 2 \\
\hline I & Das ist nichts für meine Altersgruppe & 1 & 2 \\
\hline J & $\begin{array}{l}\text { Es gibt in meiner Nähe keine für mich attraktiven } \\
\text { Möglichkeiten, ehrenamtlich zu arbeiten }\end{array}$ & 1 & 2 \\
\hline
\end{tabular}


